\title{
Computational study of comprehensive methods of initiating phase separation within metastable regions and obtaining some notable patterns in unstable region
}

\author{
by \\ Viet-Nhien Tran Duc \\ Bachelor of Science \\ University of Alberta, Canada, 2015 \\ A thesis \\ presented to Ryerson University \\ in partial fulfillment of the \\ requirement for the degree of \\ Master of Applied Science \\ In the program of \\ Chemical Engineering \\ Toronto, Ontario, Canada, 2019 \\ (C) Viet-Nhien Tran Duc, 2019
}




\section{Author's Declaration}

I hereby declare that I am the sole author of this thesis. This is a true copy of the dissertation, including any required final revisions, as accepted by my examiners.

I authorize the Ryerson University to lend this dissertation to other institutions or individuals for the purpose of scholarly research.

I further authorize Ryerson University to reproduce this thesis by photocopying or by any other means, in total or in part, at the request of other institutions or individuals for the purpose of scholarly research.

I understand that my dissertation may be made electronically available to the public. 


\section{Acknowledgement}

I would like to express my gratitude towards my supervisor, Dr. Philip Chan, who supported me throughout the course of graduate school by providing the opportunity to have fruitful discussions.

My appreciation also goes out for Dr. Philip Chan for providing me stipend and an excellent research environment.

I would like to thank Ryerson University for supporting this research with necessary funding and resources to carry out this research. 


\title{
Abstract
}

\section{Computational study of comprehensive methods of initiating phase separation within metastable regions and obtaining some notable patterns in unstable region}

\author{
Viet-Nhien Tran Duc \\ Dapartment of Chemical Engineering, Ryerson University. \\ 350 Victoria Street, Toronto, Ontario, M5B 2K3 Canada.
}

Binary phase polymer solution is interesting in that they expresses double-well local energy behavior, which means phase separation is preferred when condition is right. It is a feature that has been used to fabricate functional polymeric materials such as PDLC films for electro-optical devices (e.g. flat-panel displays and switchable windows). A uniformly mixed solution may be in one of three state: unstable, stable, or metastable. If the solution is unstable, then phase separation is spontaneous and proceeds by spinodal decomposition. If the solution is metastable, then the solution must overcome certain activation barrier for phase separation to proceed spontaneously. The activation barrier is usually the thermal noise or the fluctuation created by some external influence. This mechanism is called nucleation-and-growth. Manipulating morphology of phase separation has been of some great research interest because of its practical use. While spinodal decomposition has been well-studied, there are several other methods to further control morphology. For this thesis, the following methods are considered: double quench, anisotropic quenching with varying temperature or polymerization, surface-directed wetting, and concentration gradient. The methods are carried out within 
metastable or unstable regions or both. To numerically model, Cahn-Hilliard theory and FloryHuggins' theory are used. This thesis is to also demonstrate that, present numerical method is very efficient and can work on complex geometry. 


\section{Table of contents}

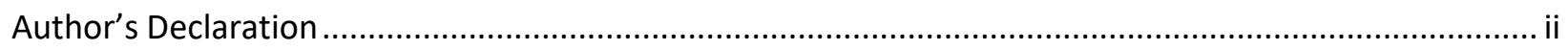

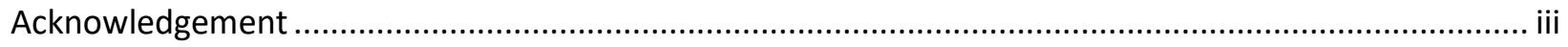

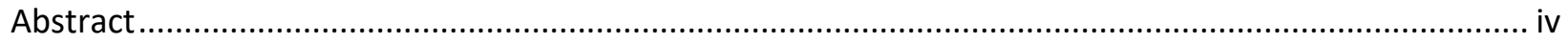

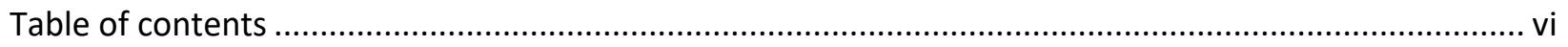

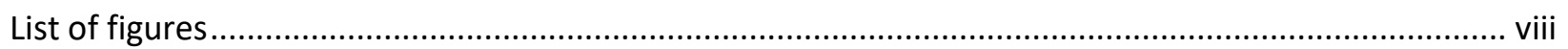

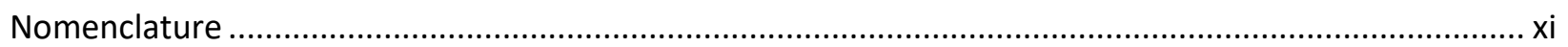

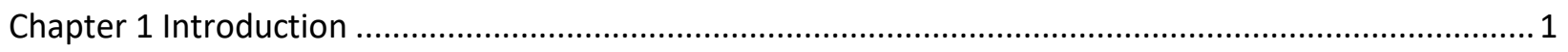

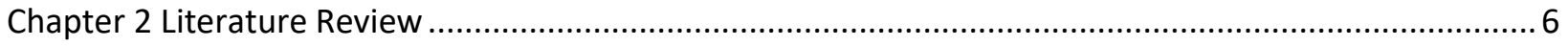

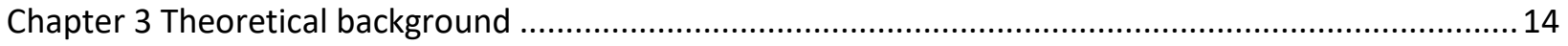

3.1 Flory-Huggins theory: Unstable, Metastable, and Stable solution ........................................ 15

3.2 Cahn-Hilliard theory and the dynamics of binary polymer solution .........................................20

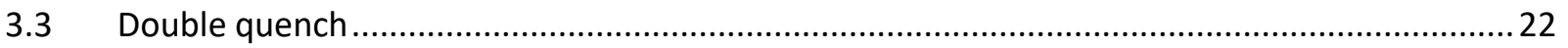

3.4 Gradient of temperature, polymerization, and concentration..................................................2 22

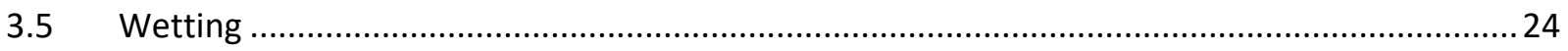

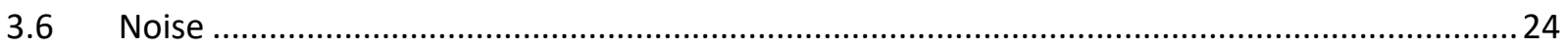

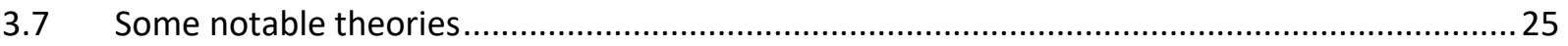

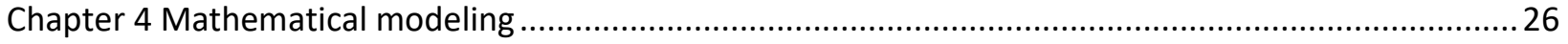

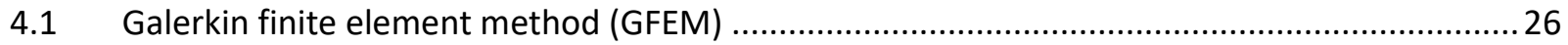

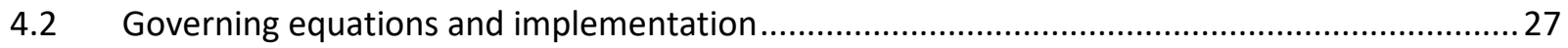

4.2.1 Governing equations, assumptions, and non-dimensionlizations ........................................ 27

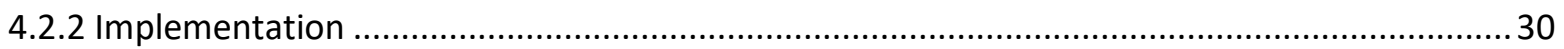

4.3 On algorithm featuring parallel computation and SuiteSparse ............................................. 33

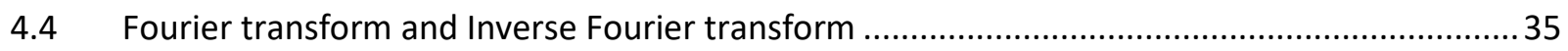

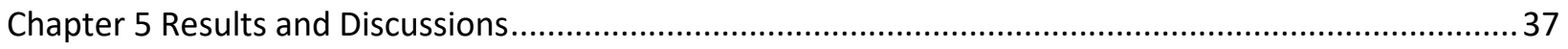

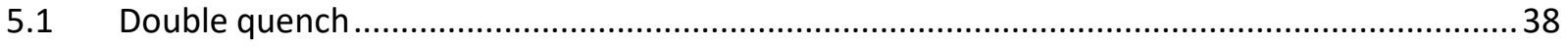

5.1.1 Unstable first quench and unstable second quench: Some remarkable patterns ....................38

5.1.2 Unstable first quench, metastable second quench ..............................................................60 


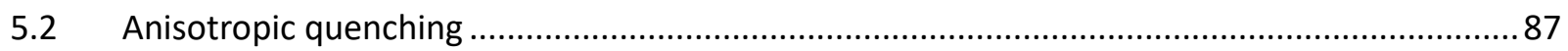

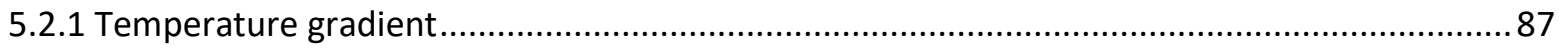

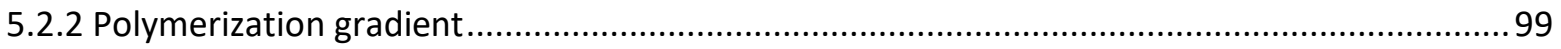

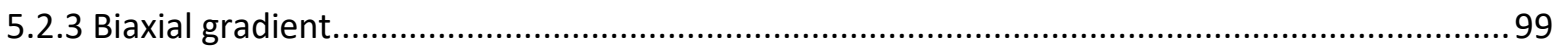

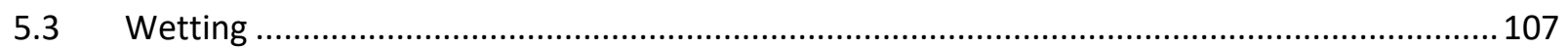

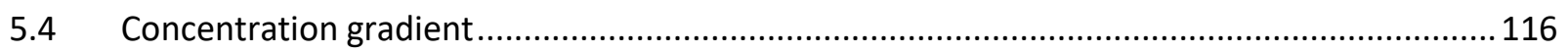

5.5 Spinodal decomposition on the complex domain with curved boundaries ........................... 126

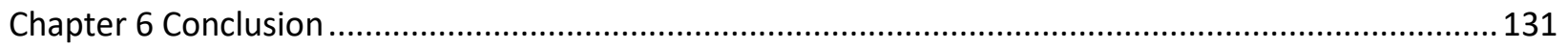

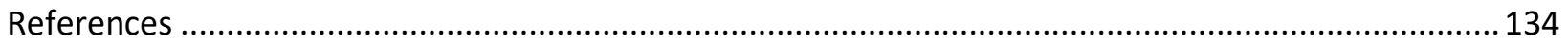

Appendix A Complimentary theoretical background and numerical methods ..................................... 140

A.1 Solution to linearized Cahn-Hilliard and determining characteristic frequency in all dimensions 140

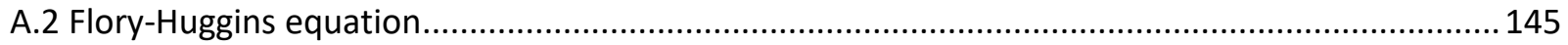

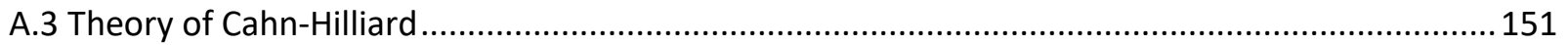

A.4 Minimum energy of a binary mixture as pointed out by C.M. Elliott......................................... 155

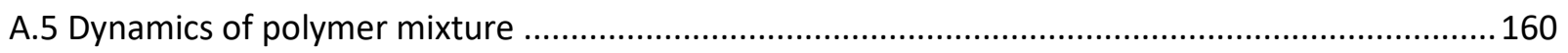

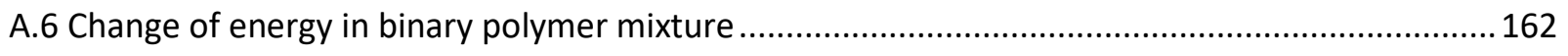

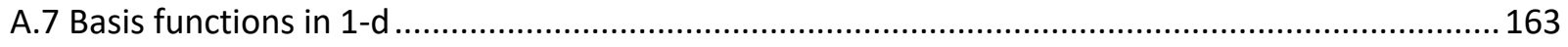

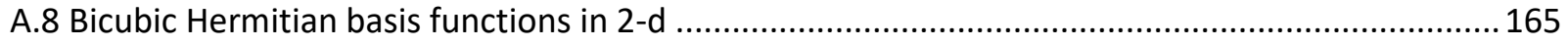

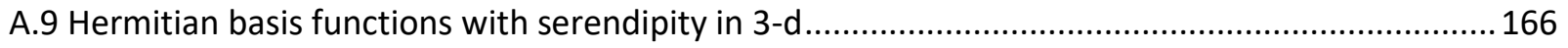

A.10 Gaussian quadrature and isoparametric mapping in 1-d ...................................................... 171

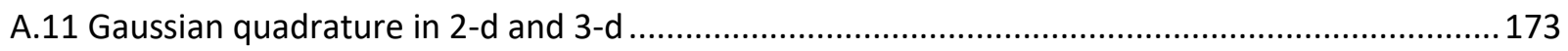

A.12 Isoparametric mapping of global basis functions from local basis functions.............................. 176

A.13 Domain with curved boundaries and irregularly shaped mesh: Demonstration with 2-d

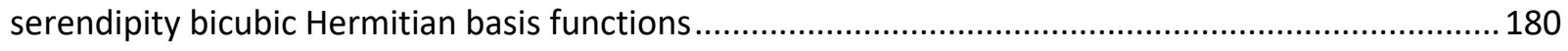

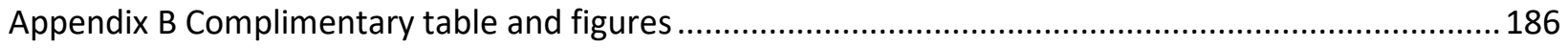




\section{List of figures}

Figure 3.1-1 The phase diagram of non-dimensionalized local free energy obtained based on equations

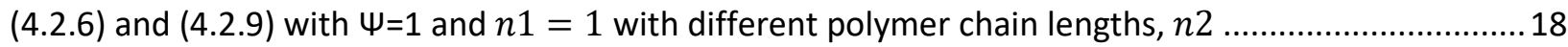
Figure 5.1-1 Concentration profile of second quench with initial configuration taken from first quench at

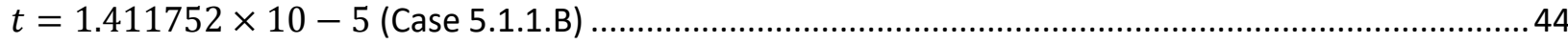

Figure 5.1-2 Contour plot of the concentration of the same simulation as in Figure 5.1-1 (Case 5.1.1.B) 45 Figure 5.1-3 Amplitude spectrum of the same simulation as in Figure 5.1-1 (Case 5.1.1.B)................... 46 Figure 5.1-4 Circularly averaged amplitude spectrum of the same simulation as in Figure 5.1-1 (Case 5.1.1.B) 47

Figure 5.1-5 Maximum of amplitude spectrum of the same simulation as in Figure 5.1-1 (Case 5.1.1.B). 48 Figure 5.1-6 Relative energy the same simulation as in Figure 5.1-1 (Case 5.1.1.B).... 48

Figure 5.1-7 Concentration profile of second quench simulation after the first quench is carried out for

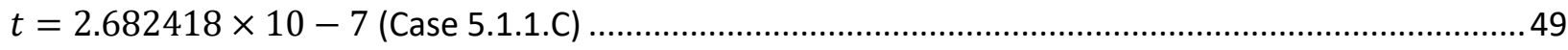

Figure 5.1-8 Contour plot of the same simulation as in Figure 5.1-7 (Case 5.1.1.C) ...............................50 Figure 5.1-9 Amplitude spectrum of the same simulation as in Figure 5.1-7 (Case 5.1.1.C).................... 51 Figure 5.1-10 Circularly averaged amplitude spectrum of the same simulation as in Figure 5.1-7 (Case 5.1.1.C) 52

Figure 5.1-11 Concentration profile with band-pass filtered applied at $t=2.136128 \times 10-7$ (Case

5.1.1.C) 53

Figure 5.1-12 Concentration profile with band-pass filtered applied at $t=2.136128 \times 10-7$ (Case 5.1.1.C) 53

Figure 5.1-13 Log plot of the concentration maximum of the amplitude spectrum of simulation in

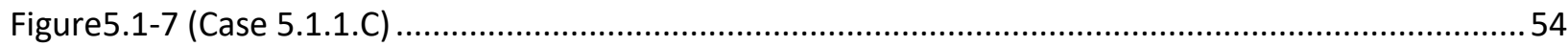

Figure 5.1-14 Relative energy of the simulation in figure 5.1-7 (Case 5.1.1.C) .......................................5 Figure 5.1-15 Concentration profile of second quench after the first quench is run until $t=5.712641 \times$

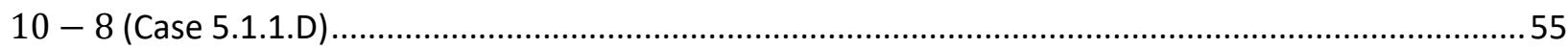

Figure 5.1-16 Contour plots of the same simulation as in Figure 5.1-15 (Case 5.1.1.D) ..........................56 Figure 5.1-17 Amplitude spectrum of the same simulation as in Figure 5.1-15 (Case 5.1.1.D) ................ 57 Figure 5.1-18 Circularly averaged amplitude spectrum of the same simulation as in Figure 5.1-15 (Case 5.1.1.D) 58

Figure 5.1-19 Concentration profile with band-pass filtered applied at $t=1.632703 \times 10-7$ (Case 5.1.1.D) .59

Figure 5.1-20 Log plot of maximum amplitude of the same simulation as in Figure 5.1-15 (Case 5.1.1.D)

Figure 5.1-21 Relative energy of the same simulation as in Figure 5.1-15 (Case 5.1.1.D) .......................60 Figure 5.1-22 Concentration profile of metastable second quench for $D=10000$, cave $=0.2, T=$ $0.29, n 1=n 2=1$ where $c o$ is obtained from Case 5.1.2.A at $t=3.264183 \times 10-5$ (Case 5.1.2.B) 65 
Figure 5.1-23 Contour plots of the same simulation as in Figure 5.2-22 (Case 5.1.2.B).........................66

Figure 5.1-24 Amplitude spectrum of the same simulation as in Figure 5.2-22 (Case 5.1.2.B)...............67 Figure 5.1-25 Circularly averaged Amplitude spectrum of the same simulation as in Figure 5.2-22 (Case

5.1.2.B)

Figure 5.1-26 Log plot of maximum amplitude of the same simulation as in Figure 5.2-22 (Case 5.1.2.B)69 Figure 5.1-27 Relative energy of the same simulation as in Figure 5.2-22 (Case 5.1.2.B)........................69

Figure 5.1-28 Concentration profile of metastable second quench (Case 5.1.2.D)................................73

Figure 5.1-29 Contour plots of the same simulation as in Figure 5.1-28 (Case 5.1.2.D) ......................... 74

Figure 5.1-30 Amplitude spectrum of the same simulation as in Figure 5.1-28 (Case 5.1.2.D) ................ 75

Figure 5.1-31 Circularly averaged amplitude spectrum of the same simulation as in Figure 5.1-28 (Case

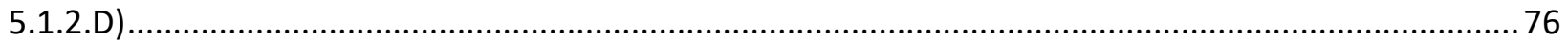

Figure 5.1-32 Band-pass filters were applied at $t=2.247859 \times 10-6$ (Case 5.1.2.D) ........................ 77

Figure 5.1-33 Log plot of maximum amplitude of the same simulation as in Figure 5.1-28 (Case 5.1.2.D)

Figure 5.1-34 Relative energy of the same simulation as in Figure 5.1-28 (Case 5.1.2.D) ...................... 78 Figure 5.1-35 Second metastable quench with 10000 , cave $=0.3, T=0.36, n 1=n 2=1$ where co is taken from configuration obtained in Case 5.1.2.C at $t=5.318622 \times 10-6$ (Case 5.1.2.E) ................81 Figure 5.1-36 Contour plots of the same simulation as in Figure 5.1-35 (Case 5.1.2.E) ......................... 82 Figure 5.1-37 Amplitude spectrum of the same simulation as in Figure 5.1-35 (Case 5.1.2.E).................83 Figure 5.1-38 Circularly averaged amplitude spectrum of the same simulation as in Figure 5.1-35 (Case

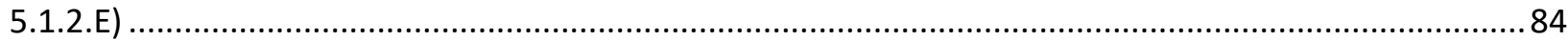

Figure 5.1-39 Band-pass filters applied to configuration at $t=8.121522 \times 10-7$ (Case 5.1.2.E)........ 85 Figure 5.1-40 Log plot of amplitudes of specific frequencies of the same simulation as in Figure 5.1-35 (Case 5.1.2.E).....

Figure 5.1-41 Log plot of maximum amplitude of the same simulation as in Figure 5.1-35 (Case 5.1.2.E)86 Figure 5.1-42 Relative energy of the same simulation as in Figure 5.1-35 (Case 5.1.2.E)..... 86

Figure 5.2-1 Concentration profile of the solution with $D=100000$, cave $=0.3, n 1=n 2=1$ and temperature varies with $T 1=0.34$ with $T 2=0.30$ (Case 5.2.1.A)

Figure 5.2-2 Two-colours contour plots of the same simulation as in Figure 5.2-1 (Case 5.2.1.A) ...........93 Figure 5.2-3 Structure factors of configurations of the same simulation as in Figure 5.2-1 (Case 5.2.1.A)94 Figure 5.2-4 Circularly-averaged structure factor of the same simulation as in Figure 5.2-1 (Case 5.2.1.A)

Figure 5.2-5 Band-pass filter is applied to configuration at $t=5.065364 \times 10-9$ of the same simulation as in figure 5.2-1 (Case 5.2.1.A) 96

Figure 5.2-6 Band-pass filter is applied to configuration at $t=3.539219 \times 10-8$ of the same simulation as in figure 5.2-1 (Case 5.2.1.A) .96 Figure 5.2-7 The variance of concentration of the concentration profiles after band-pass filters were applied at $t=5.065364 \times 10-9$ of the same simulation as in figure 5.2-1 (Case 5.2.1.A) ..... 97 Figure 5.2-8 The variance of concentration of the concentration profiles after band-pass filters were applied at $t=3.539219 \times 10-8$ of the same simulation as in figure 5.2-1 (Case 5.2.1.A) ....

Figure 5.2-9 The variance of concentration of the concentration profiles after band-pass filters were applied at $t=1.046384 \times 10-7$ of the same simulation as in figure 5.2-1 (Case 5.2.1.A) .................98 Figure 5.2-10 Log plot of maximum amplitude of the same simulation as in Figure 5.2-1 (Case 5.2.1.A). 98 Figure 5.2-11 Relative energy of the same simulation as in Figure 5.2-1 (Case 5.2.1.A). 
Figure 5.2-12 Concentration profile of the simulation with $D=60000$, cave $=0.7$ and $n 2=1$. The temperature varies in along $x$-direction with $\mathrm{T}=0.34$ at $\mathrm{x}=0$ and $\mathrm{T}=0.3$ at $\mathrm{x}=1$. Polymerization varies in $\mathrm{y}-$

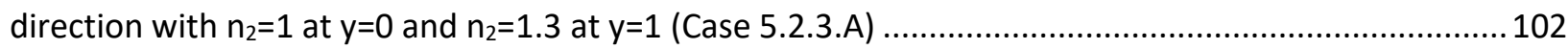
Figure 5.2-13 Two-colors contour plot of the same simulation as in Figure 5.3-9 (Case 5.2.3.A)........... 103 Figure 5.2-14 Structure factor of the same simulation as in Figure 5.3-9 (Case 5.2.3.A) .......................104 Figure 5.2-15 Circularly-averaged structure factor of the same simulation as in Figure 5.3-9 (Case 5.2.3.A) 105

Figure 5.2-16 Band-pass filters of configuration at $t=3.734029 \times 10-7$ (Case 5.2.3.A) ..................106 Figure 5.2-17 Maximum amplitude of structure factor of the same simulation as in Figure 5.3-9 (Case

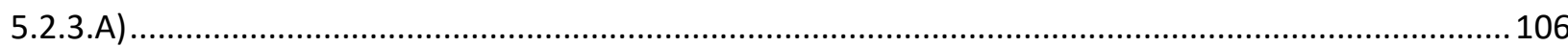

Figure 5.2-18 Relative energy over time of the same simulation as in Figure 5.3-9 (Case 5.2.3.A) .........107 Figure 5.3-1 Slice plots of relative concentration profile for $D=5000$, cave $=0.3, T=0.35, n 1=n 2=$ 1 . The wetting effect is present at all boundary surfaces with $g=0$ and $h=-1.0$. The concentration on $x y, x z$, and $y z$ planes are shown. (Case 5.3.A). 111 Figure 5.3-2 Isosurface plots of simulation shown in Figure 5.3-1. Blue surface is component-1 rich side,

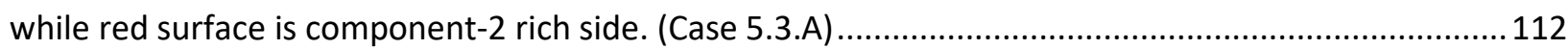

Figure 5.3-3 Structure factor of configurations shown in Figure 5.3-1 (Case 5.3.A) .............................113 Figure 5.3-4 Circularly averaged structure factor of simulation in Figure 5.3-1 (Case 5.3.A).................114 Figure 5.3-5 Isosurfaces of configuration in Figure 5.3-1 after band-pass filters are applied at $t=$ $2.864835 \times 10-5$ (Case 5.3.A).

Figure 5.3-6 The amplitudes of structure factors at specific frequencies of the simulation in Figure 5.3-1 (Case 5.3.A) 115

Figure 5.3-7 Relative energy of the process shown in Figure 5.3-1 (Case 5.3.A). 116

Figure 5.4-1 Concentration profile of the solution with $D=100000, T=0.35, n 1=n 2=1$. Initial concentration varies from $c=0.25$ at $x=0$ to $c=0.35$ at $x=1$ and also contains noise. (Case 5.4.A)

Figure 5.4-2 Contour plots of the simulation in Figure 5.4-1 (Case 5.4.A) .............................................121 Figure 5.4-3 Structure factor of the process in Figure 5.4-1 (Case 5.4.A) .............................................. 122 Figure 5.4-4 Band-pass filters of configuration at $t=3.734029 \times 10-7$ in Figure 5.4-1 (Case 5.4.A) 123 Figure 5.4-5 Band-pass filters of configuration at $t=7.239773 \times 10-7$ in Figure 5.41 (Case 5.4.A). 123 Figure 5.4-6 Concentration variance of configuration at $t=3.734029 \times 10-7$ of the same simulation as in Figure 5.4-1 (Case 5.4.A).... 124

Figure 5.4-7 Concentration variance of configuration at $t=7.239773 \times 10-7$ in of the same simulation as in Figure 5.4-1 (Case 5.4.A). .124

Figure 5.4-8 Maximum structure factor of the solution in Figure 5.4-1 (Case 5.4.A) ............................ 125

Figure 5.4-9 Average concentration along $x=0$ in the simulation in Figure 5.4-1 (Case 5.4.A)............ 125 Figure 5.4-10 Relative energy of the process in Figure 5.4-1 (Case 5.4.A) ........................................... 126 Figure 5.5-1 Concentration profile of $D=100000$, cave $=0.5, T=0.3, n 1=n 2=1$ with different $h$ values. 129 Figure 5.5-2 Contour plots of $D=100000$, cave $=0.5, T=0.3, n 1=n 2=1$ with different $h$ values. 


\section{Nomenclature}

*Some symbols in this thesis may have different meanings than as shown in the table. In such cases or if the symbols are not listed here, they are explicitly specified. Sometimes, subscripts and superscripts are not shown in discussions.

\begin{tabular}{|c|c|}
\hline$A$ & Magnitude of Fourier transform of concentration profile \\
\hline$c$ & Volume fraction or concentration of solvent \\
\hline$c_{\text {ave }}$ & Average solvent concentration \\
\hline$c_{o}$ & Initial concentration profile \\
\hline$c_{i}$ & A relevant constant with specific $i$ \\
\hline$c_{\tau}^{\omega}$ & Nodal weights of element $e$, orientation $\omega$, and type $\tau$ \\
\hline$c_{x}, c_{y}, c_{x x}, \ldots$ & Derivative of concentration with respect to components shown as subscripts \\
\hline$\hat{\hat{c}}$ & Fourier transformed concentration in $2-d$ \\
\hline$D$ & Diffusion coefficient \\
\hline$D_{T}$ & Thermophoresis coefficient \\
\hline$e$ & Identity of element \\
\hline$e_{1}$ & Equilibrium concentration of polymer-rich phase \\
\hline$e_{2}$ & Equilibrium concentration of solvent-rich phase \\
\hline$E$ & The energy of the system (J) \\
\hline$\Delta E$ & Relative energy of the system \\
\hline$f$ & Flory-Huggins free energy of mixing $\left(\mathrm{J} \mathrm{m}^{-3}\right)$ \\
\hline $\mathcal{F}$ & Fourier transformation \\
\hline$g$ & Coefficient of wetting related to concentration \\
\hline$G$ & Gibbs' free energy $(\mathrm{J})$ \\
\hline$h$ & Coefficient of wetting independent of concentration \\
\hline
\end{tabular}




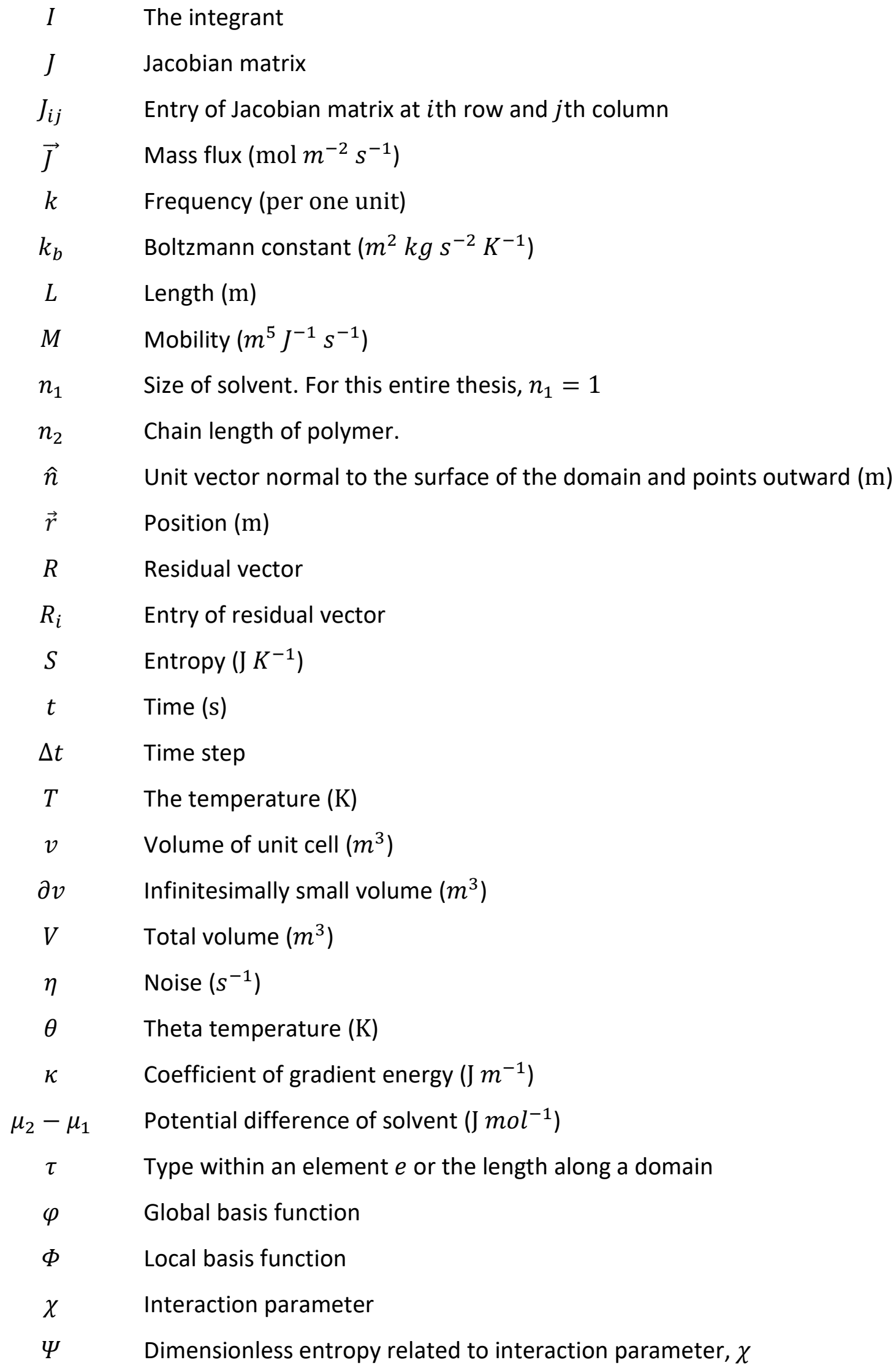


$\Omega \quad$ Domain

$\partial \Omega \quad$ The boundary of domain

$\omega \quad$ Orientation within an element $e$ 


\section{Chapter 1 Introduction}

Multiphase polymer solution has been known to express different peculiar behaviors when submitted to certain conditions. Due to the nature of the local potential energy and the law of mass conservation, binary polymer solution may have favorable lower energy when the polymer solution is phase separated rather than being in the uniform state. If phase separation from uniform state is spontaneous, i.e. it occurs without any external influence, then it is said to be in unstable region. Otherwise, if the solution has more favorable lower energy configuration but phase separation from the uniform state is not spontaneous, it is said to be in metastable state. Lastly, if the solution tends to stay in uniform state as it is the configuration that has the lowest energy, it is said to be in stable state. The process of phase separation can be unique in morphology, time of phase separation, equilibrium values, etc. Moreover, the morphology can be further manipulated by many several types of quenching and other nature of the polymer, e.g. double quench, anisotropic quench, thermophoresis, elasticity, etc.

The behaviors of multiphase polymer solution has been studied extensively from the perspectives of mathematics, physics, and material engineering. Typically, the studies are either experimental or numerical. While experimental results are direct observations of actual phenomena, numerical results can give insights into full understanding of the factors that contribute to experimental observations. Because numerical and experimental results have closely verified one another many times, it has inspired researches in both.

Numerical methods often utilize the model described in an outstanding publication by Cahn [1], recipient of National Medal of Science. This descriptions of the polymer solution has inspired many publications. In this model, the energy is often described as the integral of the entire 
volume, whose integrant is the summation of local potential energy term, which has local concentration as parameter, and gradient term, which is the absolute square of gradient of concentration with interfacial parameter. This way, the interfacial energy that exists between different phases is accounted. The change in polymer solution can be evaluated over time and space by taking functional derivative, which yields the potential difference, and continuity equation.

Because the resulting governing equation is a fourth order functional derivative, there have been difficulties in numerical computational power. In the past, due to limitation in computational power or numerical method or both, often times, linearized model is often used. Here, the governing equation is approximated with the free energy term being a scalar, as higher order term of local free energy density's Taylor expansion is dropped and approximated about average concentration. This way, only the second and fourth derivatives of concentration remains. This is a common equation that can be seen a lot in nature and solved by using wellorganized procedure in undergraduate partial differential equation course. While analytical solution can be obtained, solving it by the method of Fourier transform not only makes it a lot easier, but also let one identify important frequencies so that the numerical solution can be verified. Typical isotropic quench from uniform state into unstable state is often remarked by characteristic frequency, a sole frequency that becomes dominant in early stage. However, this form is only appropriate when the solution starts from homogeneous state and in the early stage. After this stage, the solution will enter nonlinear intermediate and late stage, which must take into account higher order term of Taylor expansion. In this thesis, the stage the solution is in at specific time frames is identified and simulation is not always started from uniform state (e.g. Second quench for double quench), thus nonlinearity of Cahn-Hilliard equation must be accounted. Especially phase separation in metastable state must be initiated with some sort of noise, of nucleation, which occurs independent of solution's free energy as described by Cahn [1]. The model that includes the noise is referred to as Cahn-Hilliard-Cook equation. Nowadays, with advance of computational technology, there have been researches that consider nonlinear Cahn-Hilliard equations, with higher dimension (2-D or 3-D in space) (e.g. [2] [3] [4]), and ever higher mesh for accuracy. However, the simulations are often still limited to high-end scientific 
computers, and it has restricted the research to only those who have access to them. In this thesis, aside from the preliminary results of binary polymer's phase separation, another purpose is to demonstrate that present model is extremely efficient and requires low computational power, hence significantly reduces the amount of computation time. In fact, all simulations were performed on standard mainstream home PC (CPU: Intel ${ }^{\circledR}$ Core $^{\mathrm{TM}}$ i7-8700, RAM: $16 \mathrm{~GB}$ ) that are available for purchase in most of computer retail stores at the time this thesis was written. First, a numerical method is deliberately selected which to reduce the fourth order problem into essentially second order problem to make it computationally less expensive. Such method has been suggested by Chan and Rey [4]. Furthermore, for simplicity of coding, all simulations were performed on MATLAB R2018a, which is available in most of comprehensive universities across North America. To take advantage of all 6 cores CPU of mainstream computer today, parallel computation algorithm is implemented, e.g. each entry of Jacobian matrix arisen from present method is computed independently as a group. To make the code as efficient as possible, repetitive computations are cut and saved to RAM in an amount that does not compromise the computational speed. Tremendous amount of credit must be given to SuiteSparse package, which is available as built-in package in MATLAB R2018a and makes research significantly more feasible. Because the method involves division of Jacobian matrix with large number of entries, extremely efficient matrix division method must be selected. Since Jacobian matrix generated tend to include mostly zeros (sparse matrix), memory can be significantly reduced by committing only non-zero entries to the memory. Tim Davis [5] has done outstanding researches in large sparse matrices that arise frequently in science, engineering, and mathematics. His algorithm is featured in many applications including Google Map. With the advance of powerful technology and extremely efficient algorithms, simulation time has been reduced from days and weeks into less than an hour for very similar model and method, and performed simulations on a standard home PC rather than high-end scientific computer with ever higher resolution.

Binary polymer may have either upper critical solution temperature (UCST) or lower critical solution temperature (LCST). A UCST phase diagram of non-dimensionalized local free energy that will be used for this thesis (see section 4.2.1) is shown in Figure 3.1-1. If a solution has 
UCST, there exists critical point at which phase separation above the temperature at this point will not occur. For example, in Figure 3.1-1, the phase diagram of polymer with $n_{2}=1$ has the peak at $T=0.4$, which is the critical temperature. If the temperature is lowered, then the solution may be either in stable, metastable, or unstable state, depending on the average concentration of the mixture. The boundary that separates these regions are binodal line and spinodal line, respectively. The opposite is true for LCST. For solution with UCST, deeper quench is carried out by lowering the temperature, while the opposite is true for LCST. For the purpose of discussion, in this thesis, we define quench depth as following: The quench is said to be deeper if the characteristic frequency computed according to A.1.22 is higher. This definition is particularly useful when the chain length of the polymer is altered, thereby shifting the phase diagram.

Thermal Induced Phase Separation Method (TIP for short) is the phase separation induced solely by bringing uniform solution's temperature into appropriate range immediately from stable state, with all other parameters constant. The morphology is dependent on depth of the quench, as identified by characteristic frequency, polymerization, and average concentration, etc. Generally, with all parameters constant, in unstable region, the deeper the mixture is quenched, the higher the characteristic frequency is. Critical quench is defined as the quench of solution whose average concentration is equal to critical point, while off-critical is defined as those that are not. Critical quench has been known to produce interconnected structures, while off-critical quenches are generally known to produce droplet-type morphology. In highly asymmetrical phase diagram, critical quench could be less like interconnected structures especially in late stage, but rather express co-continuous morphology. As mathematically proven by Amy Novick-Cohen [6], metastable and unstable solutions exhibit minimum energy when the gradient of concentration in minimal (or zero) and local concentration everywhere is either of two unique equilibrium values. Such peculiar behaviors of the solution has inspired many publications due to its contribution in practical industry.

For present model, some of the significant factors such as pressure, viscosity, elasticity, and complication that may arise from entanglement of polymers are neglected. The advantage of 
simpler model is that it is easier to study the effect of each factor and observe what each can account for. It is, however, left as future research suggestion.

In this thesis, some of published experimental observations and numerical results are closely reproduced. Behavior of phase separation using several methods are investigated, namely: double quenching, anisotropic quenching, surface-potential, and concentration gradient. The simulation is performed either in the metastable or unstable range. The mechanism that generates morphology of concentric pattern is explained by simple double quenching. Double quenching across metastable boundary is also discussed. Phase separation within metastable region is carried out by surface potential and concentration gradient. Spectacular morphology generated by temperature gradient is also discussed. Last section of Chapter 5, Section 5.5, is included to demonstrate capability of present model to efficiently simulate phase separation on complex geometries. 


\section{Chapter 2 Literature Review}

This chapter provides a literature review that will be relevant for this thesis. Purpose of this thesis is to investigate behavior of binary polymer blend's phase separation using variety of methods. For phase separation to proceed in metastable solution, the solution must achieve some configuration having an energy higher than its perfectly uniformly mixed state, creating an activation barrier. Phase separation of binary polymer blend in metastable region typically proceeds by thermal fluctuation, which acts as activation energy. As suggested by Cook [7], it is an event that may have significant influence on a course of phase separation. Though such event is random and involves extensive statistical physics, the numerical studies are often limited to simple estimations. However, statistical aspects of the polymer solutions are out of scope of this thesis. From engineering perspective, strategies to manipulate the polymer blend with well-defined methods are more of interest. Thus, instead of using the noise, polymer blend is quenched with different methods that involve metastable state in this thesis. Namely, metastable and unstable phase separation with the following methods are carried out: double quench, anisotropic quenching, wetting, and concentration gradient. In this section, each of those topics is reviewed. Some industrial applications of polymer blend that make this study valuable are also discussed.

Castro [8] reported the methods for making microporous products. Graham et al [9] monitored thermal-induced phase separation of poly(methyl methacrylate) membrane by small angle light scattering and scanning electron microscopy (SEM). Since the behavior of scattered lightintensity agreed with Cahn-Hilliard theory, the authors concluded that spinodal decomposition is the driving mechanism of phase separation. Graham's experiment was later verified numerically by Barton et al [10] with Cahn-Hilliard's equation. West [11] has described variety of methods to prepare polymer dispersed liquid crystals by using TIP and PIP (PIP stands for 
polymerization induced method. When polymerization or cracking is carried out, phase diagram is shifted, as shown in Figure 3.1-1, placing the solution in different quench region), and also described how cooling rate could be used for morphology control. Kim and Palffy-Muhoray [12] carried out polymerization within liquid crystal E-7, the epoxy Epon 828, and the curing agent Capcure 3-800. As polymerization is induced, phase separation occurs, giving rise to regions of different phases. They have associated the behavior of the system using Cahn-Hilliard theory, which makes it appropriate to infer that they have carried out PIP process. In the publication by Liu and Kiran [13], the structure factor at different points within phase diagram is described. Here, they used polydimethylsiloxane and carbondioxide. Quench depth is controlled by pressure rather than temperature, thus so-called pressure-induced phase separation. Structure factors were obtained by monitoring transmitted and scattered light intensities at different angle. In spinodal decomposition, high-amplitude structure factors form into a ring shape, while in metastable region, such ring is absent, and the amplitude monotonically decreases away from the center.

Double quench by varying the temperature was first studied by Ohnaga and Inoue [14] by 1-d numerical simulation and later in 2-d by Tran et al [15]. Kwak et al [16] also studied two-step quenching of polymer blend of polystyrene and poly(2-chilorostyrene) with di-n-butyl phthalate. This blend has LCST property. The quench was first quenched at $140{ }^{\circ} \mathrm{C}$, then put into deeper quench at $165^{\circ} \mathrm{C}$. In the first quench, large co-continuous domains were created. In the second quench, smaller droplets emerged within these domains then grew in size, but eventually started disappearing until none was left. At the same time, larger co-continuous domain continues to enlarge. Hajime [17] performed several double quenching methods by either raising or lowering the temperature within unstable region for binary mixture of styrene and poly(vinyl methyl ether), which expresses LCST property. For a deeper quench (higher temperature), new structures evolve within larger domains from the first structures. On the other hand, shallower quench (lower temperature) leads to long range interface instability. Cong et al. [18] have shown that periodic forcing gives spinodal ring that is sharper in width as compared to that of continuous forcing by using photo-cross-linking of anthracene-labelled polystyrene/poly(vinyl methyl ether. 
Harada and Cong [19] noted the complexity that arises when cross-linking a polymer blend in the miscible region. For their experiment, a mixture of poly(styrene-stat-(chloromethyl)styrene) (PSCS) and poly(vinyl methyl ether) (PVME) were used. Anthracene is added to PSCS as a crosslinker. As the mixture is photo-cross-linked from miscible region, phase diagram gradually shifts the mixture into unstable region, but with some complexity. When cross-linking is started at the vicinity of co-existence curve (binodal line), a typical behavior spinodal decomposition is observed, as interconnecting structures arise. Dominant frequency increases with time then becomes stationary later. When photo-cross-linking is started at the intermediate point between binodal curve and glass temperature, three scenarios arise: Labyrinthine structures, nucleation-assisted spinodal structures, and concentric pattern. They reasoned that, under cross-linking reaction, it causes complexity in long-range interaction. Some complications include, just to mention a few, inhomogeneous cloudiness within the solution limit incoming intensity of the light, entanglements of the particles, the shrinkage of the volume, and the change in elasticity. Thus, formation of morphology is a result of competition between chemical reaction and phase separation of polymer blends. In an attempt to numerically model the observation, Furukawa [20] numerically produced concentric pattern by passing the solution into metastable region then into unstable region. When in metastable regions, nucleation occurs, which will act as scaffolds for generating concentric pattern when the solution enters unstable region. However, nucleation and growth is generally slow because of viscosity, and it depends heavily on large fluctuation within solution, thus phase separation may not be as vigorous as that for spinodal decomposition. For this reason, Okada et al. [21] later proposed a numerical model which can produce concentric pattern without relying heavily on nucleation and growth mechanism. Here instead, they argued that, under continuous quenching where molecular weight of one component increases by cross-linking, mobility changes with time. Specifically, the mobility is made to decrease over time while cross-linking takes place. They also made the skewness of local free energy diagram, which is related to molecular weight of one of the components in the blend, dependent with time. By choosing the right timedependent mobility, they were able to reproduce concentric pattern using only spinodal decomposition, thus nucleation and growth is no longer a prerequisite for concentric pattern. Kyu and Lee [22] have monitored phase separation in a polymerizing system. Sample used was 
carboxyl terminated polybutadiene acrylonitrile/epoxy/methylene dianiline. Here, methylene dianiline is a curing agent. They noticed that, at first, there is a shift in characteristic frequency toward higher side. As the solution enters into late stage, this characteristic frequency shifts in the opposite direction, towards the lower side. This shift seems to be because as polymerization proceeds, the phase diagram shifts, putting the solution in deeper quench. They have verified such observation using Cahn-Hilliard model incorporating Flory-Huggins-deGenes theory with time-dependent polymerization. Hirose et al. [23] obtained bicontinuous gradient morphologies in polymer blend of Poly(ethyl acrylate)/poly(methyl methacrylate) (PEA/PMMA). Polymerization of MMA monomers is a process that depends on the intensity of the light. When exposed to light, it expresses auto-acceleration behavior, or Trommsdorff-Norrish effect. Simply put, it is the time when polymerization happens very quickly. The higher the intensity, the slower this time is. The morphology of the sample can be frozen after this time. This way, by creating a gradient of light intensity within the domain, different morphologies can be obtained. By using two different methods of employing light gradients in different directions on the same sample, they obtained biaxially co-continuous gradient morphologies.

Enge and Kohler [24] investigated thermophoresis in critical polymer blend for the first time. In their experiment, temperature gradient was applied to a sample of poly(dimethyl siloxane) and poly(ethyl-methyl siloxane). By employing holographic grating technique, thermal diffusion was monitored closely. They have concluded that even a small fluctuation in temperature differences can lead to significant change in composition. Kumaki et al [25] studied the effect of temperature gradient on a ternary polymer blend of polystyrene-polybutadienedioctylphthalate in stable region. This polymer blend has shown spinodal-like behavior with emerging characteristic wavelength. The reasoned that temperature gradient has induced concentration gradient by thermophoresis. Typically, larger species migrate towards cooler surface. Concentration gradient established initiates phase separation.

The importance of the rate of quenching is discussed in Furukawa's numerical simulation [26]. In his numerical investigation, the boundary that separates miscible and immiscible regions of polymer is shifted at different rates. Sufficiently high rate yields irregular morphology that is normally observed in typical spinodal decomposition, but with varying structural size across the 
domain. In other cases, two different regular morphologies can be obtained: For some moderate speed, a lamellar morphology parallel with the boundary results. For some slow speed, lamellar morphology orthogonal to the boundary is observed. They also observed other intermediate patterns. Okinaka and Tran-Cong [27] studied polymer blend of poly (2chlorostyrene)/poly (vinyl methyl ether)(P2CS/PVME) under temperature gradient. One side of the sample is stationed in miscible region, while the other is in immiscible region, thus there is a boundary that separates these two regions. As the temperature gradient is gradually increased, this boundary shifts towards miscible side, widening unstable region. He noted that the inhomogeneity in characteristic frequency exists in the mixture. The characteristic wavelength in the direction of the temperature gradient is smaller than that in the direction perpendicular to the temperature gradient. Their results are in agreement with Furakawa's numerical investigations. In another similar publication [28], they called the resulting morphology spatially graded structures. In the vicinity of the boundary that separates stable and unstable regions, interconnecting structures evolve. Meanwhile, in the deeper quench regions, the droplets have formed and are evolving with time because they have entered into late stage.

Numerical study of spinodal decomposition under the influence of thermophoresis was done by Kukadiya et at [29]. As the thermophoresis, which directs the mass-flux by temperature gradient rather than random noise, and spinodal decomposition, which creates random structures by random fluctuation, compete with one another, when the quench is deep, the effect of spinodal decomposition hinders the effect of thermophoresis, though the rate of early stage is somewhat faster. Anders and Weinberg [30] numerically investigated the effect of thermophoresis by applying sinusoidal temperature gradient to polymer blend and observed that random structure is most prominent at first, then the phases gradually align with the temperature gradient, i.e. thermophoresis has prevailed. When local heating is applied at the center of the domain, spinodal structure evolves around locally heating area, creating concentric-like pattern. The structures further away from it are more random. Their numerical research was partially motivated by the study by Voit et al [31]. In their experiment with poly (dimethyl siloxane)/poly (ethyl-methyl siloxane), they have shown that phase separation pattern can be manipulated locally by taking advantage of thermophoresis. To create 
temperature gradient, laser beam was focused at the center of the sample, which has been exposed to either stable or unstable temperature. Here, laser beam brings the exposed region's temperature higher into stable region. For the former case, a ring pattern results around the focused beam. After laser beam is turned off, this ring pattern somewhat enlarges and faded. When the laser beam was focused on unstable sample that had reached intermediate stage then turned off, rim-like structure persisted for long time. Numerical study revealed that, only when thermophoresis is accounted, the experimental observation can be closely reproduced. Without thermophoresis, the sample simply returns to randomly distributed interconnecting structures. Lee et al [32] have developed a computational model to study phase separation under linear temperature gradient. In their findings, anisotropic morphology was obtained. The phase separation starts from deeper quench side and proceeds towards shallower quench region. The time it takes to reach the beginning of the intermediate stage is longer in shallower regions, creating a wave of phase separation. Droplet size and density increase with shallower quench during the intermediate stage of spinodal decomposition. The shape of the droplet is the same throughout the entire domain.

In nature, materials may manifest special intermolecular interactions with the surfaces they bind to. It is especially useful in generating a desired pattern by using the surface, making it an attractive topic in nanotechnology. When the material possesses wetting property, it creates a concentration gradient immediately adjacent to wall it attaches to. This behavior is typically numerically modeled as a linear equation with respect to concentration for a polymer blend. A numerical model and results were described in publication by Puri and Frish [33]. Later, a critical review of surface-directed spinodal decomposition was also done by them [34]. Tanaka [17] investigated the case in which glass particles, which has preferential affinity towards one component, are inserted into a polymer blend of styrene (OS) and $\varepsilon$-Caprolactone (OCL). When the particles are immobile, a bridge of OCL-rich phase forms between particles that are close enough. When the particles are mobile and the density is low, spherical particles segregate into the droplets to reduce interfacial energy. As the density of the mobile particles is increased, finer interconnected structures form. 
Yan and Xie [35] numerically studied double quench under the influence of wetting. The first quench with surface-potential but different quench depths lead to different morphologies especially within the bulk, thereby changing the course of competition between surface potential and chemical potential due to random fluctuation within the solution. They have also observed the coarsening of lamellar structures created by surface potential. Yan and Xie [36], by using 3-d numerical model, showed that lamellar morphology can be generated when the effect of surface potential is more significant than the potential of concentration fluctuation i.e. when the quench depth is shallow and parameters for the surface is made to raise the surface potential high. The lamellar structures follow logarithmic law in the early and intermediate stages. Numerical studies on strip-patterned surface-directed spinpdal decomposition was carried out also by Yan and Xie [37]. A three dimensional model has allowed them to study the behaviors within the bulk and at the surface at the same time. As the stripe patterns emerge at the surface, a check-board pattern is seen from side view (the face that has no wetting effect and is orthogonal to wetting surface). Such check-board pattern seems to have evolved as the effect wetting stripe patterns have on the wetted surface as it propagates into the bulk, causing several phase inversions. Yan and Xie [38] numerically investigated surface-directed phase separation when minority or majority component is preferred by the surface. When minority component is preferred, a wetting layer may display pure diffusion limited growth law, logarithmic growth law, or Lifshitz-Slyozov growth law. On the other hand, when majority component is preferred, growth is always logarithmic. Surface induced nucleation can be observed within certain range of quench depth. Shang et al [39] has incorporated elastic energy term into model and highlighted its important role in surface-directed spinodal decomposition. In the presence of isotropic elastic energy, check-board pattern in the side view of the solution seems to persist longer. Lee et al [40] performed a simulation in which spinodal decomposition is carried out with immobile particles (spheres, fibers, platelets), which they called filler. Such particle has preferential attraction to one component. In near critical phase separation, concentric pattern evolves as the rings around the filler particles emerge and propagate away from it. Over time, in the late stage, concentric pattern is destroyed and overtaken by random interconnected structures for critical quench. In their publication, they also noted the significance of the noise. Typically, larger noise makes random interconnected structures more 
significant than the effect of wetting, while smaller noise allows the concentric pattern to propagate larger. They have also studied the magnitude of the affinity towards filler particle. In conclusion, the larger the affinity is, the larger the concentric pattern can propagates away from it. Their numerical study reconciles with the experimental observation by Karim et al [41], where silica particles are inserted into polystyrene and poly(vinyl methyl ether) blend to generate circular composition wave, or target pattern about the filler particles. Tabatabaieyazdi et al [42] have studied phase separation under temperature gradient and surface-potential.

There has been studies in which the concentration gradient is established prior to quenching. This is normally achieved by evaporating the solvent locally. Jayalakshmi et al [43] studied phase separation of the mixture that has concentration gradient by using Helma quartz cell. They have deliberately set up the cell so that the critical quench region lies in the center of the domain. The result was the interconnected structures in the central regions and droplet structures at two ends. The phase separation occurred first in the central region. This is expected because it is the region with the deepest quench. Numerical studies on concentration gradient with non-uniform initial concentration was carried out by Lacasta et al [44]. Observations similar to experimental results were obtained i.e. convoluted structures in critical regions and droplet structures in off-critical regions. In Chan's numerical study in 1-d with concentration gradient [45], graded droplet size is distributed along the domain. In Matsuyama's experiment [46], a mixture of isotactic polypropylene and diphenyl ether is prepared with ether being the diluent. Concentration gradient is created by evaporating diluent from the surface. After phase separation, varying pore sizes were observed along the surface of the sample.

Because desired shape and size of pores can be obtained in the lab, phase separation of polymer blends has several industrial applications. One example is the porous membrane filter [47] [48] [49] [50]. It has also been used as catalyst for chemical reactions [51] [52]. It can be used to manufacture paper, paint, and pharmaceutical industries [53] [54], energy storage devices [55] [56], electro-optical devices [57] [58], biophysics [59] [60] [61], nanocomposite materials [62] [63], and scaffolds for tissue engineering [64]. These porous materials can be used for electrode of lithium-ion battery [65] [66]. There are many other applications as well. 


\section{Chapter 3 Theoretical background}

This chapter gives a brief overview on the background on the dynamics of binary polymer solution, which is the primary concern of this entire thesis. Phase separation is the de-mixing of multi-components polymer solution into different phases with higher concentration of a component. This may occur with the external force or spontaneously by simple diffusion. Demixing occurs spontaneously because the phase separated state is thermodynamically favorable. The Flory-Huggins theory is excellent for describing this phenomena. While the FloryHuggins theory successfully describes local potential energy of multi-components polymer solution, Cahn-Hilliard theory takes into account the immediate environment of the solution, hence the thermodynamics of the entire solution within a confined volume. These two theories are used together for study of nonlinear Cahn-Hilliard equations. Using Flory-Huggins theory is appropriate in that it accounts for the temperature and the polymerization, or chain length, of each polymer because this thesis involves the study of the solution under the influence gradient(s) of the temperature and polymerizations. The derivations of the equations are included in Appendix $A$ as appendix for neatness (Though the readers are still recommended to refer to original citations). Ever since these excellent theories are published, several more publications have also been published to better them. In this chapter, only some of the important equations and their features are mentioned, which is suffice for creating model for this study. Here, phase separation is defined for the convenience of the discussion. Phase separation is said to be occurring if:

$$
\frac{\partial}{\partial t} \int_{V}\left(c-c_{a v e}\right)^{2} \partial v>0
$$


Where $\mathrm{c}$ is the concentration of solvent, $c_{\text {ave }}$ is the average of such, and $t$ is the time. This means that, phase separation is said to be occurring if the variance of concentration increases with time.

\subsection{Flory-Huggins theory: Unstable, Metastable, and Stable solution}

Flory-Huggins' theory attempt to describe the dynamics of polymer using finite lattice cell model. The entropy is obtained statistically by fitting solvent and polymer molecules into the cells. The enthalpy is obtained by relative energy difference of newly formed molecular interactions between two different species as compared to those between the same entities. It involves dimensionless parameter, $\chi$, which can be obtained experimentally. More detailed derivation is shown in Appendix A. In this section, some of the important features of the theory are pointed out. Discussion is limited to only upper critical temperature solution.

By Flory-Huggins theory, the local free energy of a binary solution is given by:

$$
f=\frac{k_{b} T}{v}\left(\frac{c}{n_{1}} \ln c+\frac{1-c}{n_{2}} \ln (1-c)+\chi \frac{c(1-c)}{n_{1}}\right)
$$

Where $k_{b}$ is Boltzmann constant, $T$ is the temperature, $\mathrm{v}$ is the volume of unit cell, $c$ is the concentration of component 1 (which is the solvent for this thesis), $n_{1}$ is size of one molecular of component 1 , and $n_{2}$ is the size of one molecule of component 2 (which is the polymer in this thesis). Here, the concentration is defined as volume fraction of the component, thus it is dimensionless. For this thesis, $n_{1}=1$ for all simulations (Later on, it can be seen that keeping $n_{1}=1$ is appropriate as dimensionless parameter, $\mathrm{D}$, can account for the size of the solvent. Thus, $n_{2}$ is really the ratio of the size of the polymer to that of the solvent molecule). Here, $\chi$ is the dimensionless parameter that is usually obtained experimentally by fitting the curve (e.g. See [67]). For UCTS, it often takes the form: 


$$
\chi=c_{1}+\frac{c_{2}}{T} \text { where } c_{1}, c_{2} \in \mathbb{R}
$$

Sometimes, it shows some nonlinearity as following:

$$
\chi=c_{1}+\frac{c_{2}}{T}+\frac{c_{3}}{T^{2}} \text { where } c_{1}, c_{2}, c_{3} \in \mathbb{R}
$$

It has also been shown that $\chi$ is a function of concentration in some cases. For this entire thesis, the format of (3.1.2) is considered. More specifically:

$$
\chi=\frac{1}{2}-\Psi\left(1-\frac{\theta}{T}\right)
$$

Where $\Psi$ is the dimensionless entropy, $\theta$ is the theta temperature, at which the solution behaves ideally.

Remarkable feature of this equation is that it has a double well-potential form for low temperature range, which is often observed for multi-phase solution. It means that there exists a line tangent to only exactly two different points of different concentrations. When no mass flux boundary condition and zero wetting boundary condition are imposed to a system of binary solution, it can be proven that if the solution with average concentration between these two tangent points completely separates into either of these two concentrations, then the solution has achieved the minimum energy and thus is in stationary equilibrium, as shown by Elliott [68]. The proof is also shown in the appendix, A.4. These two concentrations are referred to as equilibrium concentration $1, e_{1}$, and equilibrium concentration $2, e_{2}$, where $e_{2} \geq e_{1}$. Between $e_{1}$ and $e_{2}$, there exists two separated regions in which $\frac{\partial^{2} f}{\partial c^{2}}>0$ and one region with $\frac{\partial^{2} f}{\partial c^{2}}<0$ in between them. It can be proven, by several different methods, that when uniformly mixed solution has an average concentration so that $\left.\frac{\partial^{2} f}{\partial c^{2}}\right|_{c=c_{\text {ave }}}<0$, phase separation is spontaneous, as larger fluctuation yields lower energy. Here, any small fluctuation can initiate phase separation and the process is referred to as spinodal decomposition, and the solution is said to 
be in unstable state. In section A.1., the idea is proven using growth factor obtained by solving partial differential equations with Fourier transform, i.e. growth factor as shown in equation (A.1.15) is always positive as long as $\left.\frac{\partial^{2} f}{\partial c^{2}}\right|_{c=c_{a v e}}<0$, leading to phase separation. On the other hand, it can also be proven that if the solution has an average concentration so that $\left.\frac{\partial^{2} f}{\partial c^{2}}\right|_{c=c_{\text {ave }}}>0$, any small fluctuation is not preferred thermodynamically hence phase separation is not spontaneous. However, as long as the average concentration is between two equilibrium concentrations, there exists configurations that conform energy lower than the solution in uniformly mixed state. Thus, if fluctuation is sufficient large, the solution can achieve a state in which phase separation proceeds, forcing every point of the solution to approach equilibrium concentrations. The need of initial fluctuation for phase separation can be thought as activation barrier. Because of its partially stable and partially unstable nature, the solution is said to be metastable. This initial fluctuation required for phase separation can be initiated by some other source independent of the diffusion, such as thermal noise. When sufficient fluctuation is achieved, it is said the nucleation has formed. Then, the nucleus grows thermodynamically, and this process is call growth. Together, it is referred to as Nucleation and Growth (NG) process. Finally, if the solution has an average concentration lower than $e_{1}$ or higher than $e_{2}$, uniformly mixed state is always preferred, and the solution is said to be stable. 


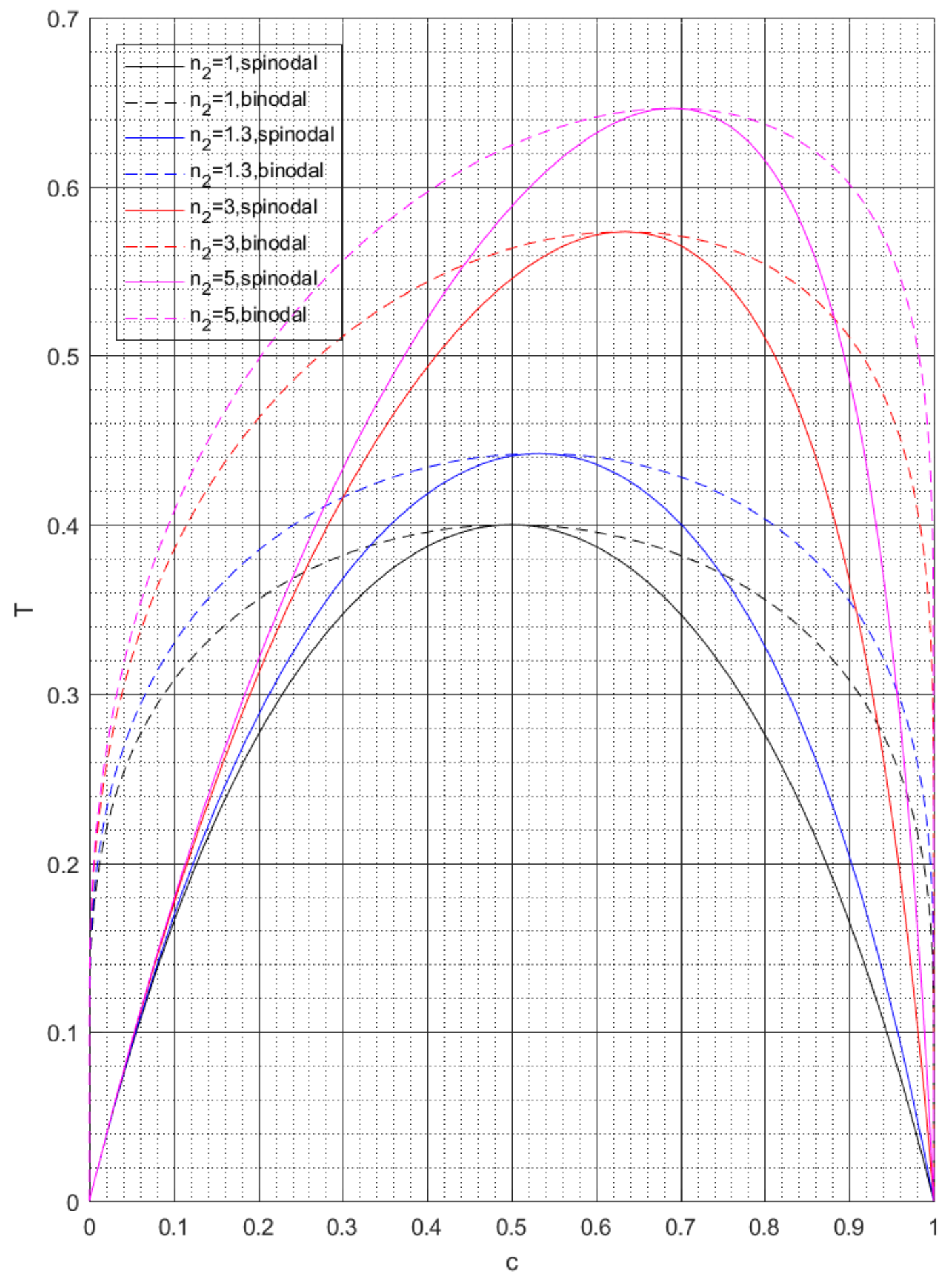

Figure 3.1-1 The phase diagram of non-dimensionalized local free energy obtained based on equations (4.2.6) and (4.2.9) with $\psi=1$ and $n_{1}=1$ with different polymer chain lengths, $n_{2}$ 
In order to recognize the state of the solution, it is convenient to draw a phase diagram, which outlines the boundaries between 3 different regions. Recall that stable and metastable solution are distinguished by equilibrium value, $e_{1}$ and $e_{2}$, while metastable and unstable solution are distinguished by $\frac{\partial^{2} f}{\partial c^{2}}$, thus two inflection points where $\frac{\partial^{2} f}{\partial c^{2}}=0$ need to be identified. Each of these boundaries shift as the temperature is brought from zero to higher values. Eventually all these boundaries merge at critical temperature, $T_{c}$. The concentration where all these points merge is referred to as critical concentration, $c_{c}$. Another important features of Flory-Huggins equation is the skewness created by increasing $n_{2} \geq 1$. For this entire thesis, all simulations are done with $n_{1}=1$. The phase diagram is perfectly symmetrical when $n_{2}=1$, but as it increases, the phase diagram skews rightwards. Figure 3.1-1 shows some phase diagrams at different values of $n_{2}$. The inflection points form spinodal line, while the equilibrium points form binodal line. (It is called so because of double-well shape). The region within spinodal line is unstable where uniform solution undergoes spinodal decomposition with any small fluctuations present, the regions engulfed between spinodal and binodal lines are metastable where nucleation and growth mechanism occurs if nucleation is sufficient, and the region outside binodal line is stable, where the solution tends to stay in uniform state. In this thesis, different values of $n_{2}$ are used. To identify in which region the solution lies, the readers are encouraged to refer to phase diagram in Figure 3.1-1 and Table $B-1$, which shows characteristic frequency, $f_{c}$, if the solution is unstable. When the temperature or the polymerization is shifted suddenly, the solution will experience new environments and behaves differently. When the temperature or polymerization gradient is established, different region of solution experiences different driving force. These are some of the interests of this research. At this point, quench depth within unstable region is defined by relating it to signature structure of the solution as following. First, when a uniformly mixed solution is mixed and is subject to unstable region, structure with unique frequency, which is referred to as characteristic frequency, $f_{c}$, emerges in the early stage. This is discussed in more details later and the derivation is shown in section A.1 as Appendix. Now, the solution is said to be in deeper quench if it yields higher characteristic frequency. By equation (A.1.22), on spinodal line that separates metastable and unstable regions, $f_{c}=0$. It is easy to recognize that, with all parameters constant, the lower the 
temperature, the deeper the quench is. This definition is useful when polymerization is shifted, resulting in different phase diagram. $f_{c}$ for the model used in this thesis can be analytically computed using (A.1.22) as derived in appendix, A.1, allowing us to validate the results of simple spinodal decomposition.

\subsection{Cahn-Hilliard theory and the dynamics of binary polymer solution}

Thermodynamics of multi-phase polymer solution can be well described by accounting for the local energy and its immediate environments. Cahn and Hilliard [1] have done pioneering work by considering Taylor expansion of local energy with concentration and its derivatives as independent terms. Appropriate assumptions, boundary conditions, and approximations are applied to obtain the energy of the solution.

$$
E=\int_{\Omega}\left(f+\kappa|\nabla c|^{2}\right) \partial v
$$

Here, $f$ is the local free energy of the solution and $\kappa$ is the positive coefficient related to energy of gradient of concentration. It has been suggested that $\kappa$ is a function concentration, most notably by de Gennes [69] (Which is equivalent to Flory-Huggins-de Gennes containing absolute of gradient term). However, commonly used formulation is based on linear approximation and makes the simulation with present model significantly slower especially in late stages of phase separation. For this reason, it is assumed to be constant for this entire thesis, which is a reasonable approximation for many studies. The potential difference in the solution is obtained by taking functional derivatives of the above with respect to concentration, which varies with position.

$$
\mu_{2}-\mu_{1}=\frac{\delta E}{\delta c}=\frac{\partial I}{\partial c}-\nabla \cdot \frac{\partial I}{\partial(\nabla c)}+\cdots=\frac{\partial f}{\partial c}-2 \kappa \nabla^{2} c
$$

Mass flux of a solution is related to potential by Fick's law: 


$$
\frac{\partial c}{\partial t}=-\nabla \cdot \vec{J}=\nabla\left[M \nabla\left(\mu_{2}-\mu_{1}\right)\right]
$$

Where $M$ is mobility and is positive. In this thesis, it is assumed to be constant. That is to say, the effect of the temperature on the local free energy of solution, as shown in Flory-Huggins theory, is more significant that that of the mobility. By continuity of solution:

$$
\frac{\partial c}{\partial t}=-\nabla \cdot \vec{J}=\nabla\left[M \nabla\left(\mu_{2}-\mu_{1}\right)\right]
$$

By combining equations:

$$
\frac{\partial c}{\partial t}=\nabla \cdot\left[M \nabla\left(\frac{\partial f}{\partial c}-2 \kappa \nabla^{2} c\right)\right]
$$

For a closed system, there must be no mass-flux at the boundaries. Also, no wetting boundary condition must be applied in order to make solution unique [1] [68]:

$$
\begin{aligned}
& \vec{J} \cdot \hat{n}=0 \text { on } \partial \Omega \\
& \nabla \mathrm{c} \cdot \hat{n}=0 \text { on } \partial \Omega
\end{aligned}
$$

This is the dynamics of the solution. Here, note that $f$ must satisfy certain conditions characteristic of multi-phase polymer solutions, especially the double well potential form. A simple polynomial is usually suffice for many studies. However, here, Flory-Huggins theorem is used to account for the chain length of the polymer. As proven in section A.6, the overall energy of the solution will always decrease without any external force. Elliott [68] has shown that, given all the boundary conditions above, the solution is unique, and that there exists a minimum energy for a solution with specific parameters. In unstable region, the solution will always tend to this energy level, while metastable solution requires some initial fluctuation. If the solution is quenched from uniformly mixed state into unstable region, then a structure with specific frequency, referred to as characteristic frequency, $f_{c}$, emerges quickly. This is 
usually experimentally observed and can also be mathematically computed using linearized Cahn-Hilliard equation. This equation is derived for present model in section A.1 and as shown in equation (A.1.22). In experimental settings, it is measured by light scattering method. It is useful for describing structures quantitatively and identifying in which stage the solution is in, and therefore will be used intensely for this study.

\subsection{Double quench}

Double quench is done by first allowing the solution to diffuse with desired parameters, then one or more parameter is changed quickly so that newly established solution now experience different driving force. If the change of the parameter is not quick enough but rather gradual, then it is a continuous quench and is not considered in this thesis. In double quenching studies, the solution is always in isotropic condition, thus any local variation within the domain except at and near the boundaries are not expected because all areas experience the same driving force. More specifically, the double quenches by temperature change or polymerization/cracking are investigated. In this thesis, it is numerically done by first letting the solution phase separates under certain conditions, then the configuration is saved and used as the initial configuration in the next simulation with new parameter. This can yield several interesting morphological results, which will be shown in Chapter 5.

\subsection{Gradient of temperature, polymerization, and concentration}

Creating a gradient of temperature or polymer chain lengths or both within a domain leads to isotropic quenching, i.e. different regions of the solution diffuse by different driving force. Experimentally, since it is done on micro-scale domain, careful experimental set-up is required. Nonetheless, there have been several experimental studies on them. Temperature gradient is straightforward as it does not change the property of the polymers within the solution. As can be seen in Flory-Huggins equation, local energy of the solution is dependent on temperature. 
Thus, when continuity equation is studied, the gradient and Laplacian terms must be expanded deliberately, as seen in the study done by Lee [32]. Also, Ludwig-Soret effect, or thermophoresis, has been identified in binary polymer solution experimentally [24]. This is another driving force different from spinodal decomposition that dictates the morphology of the solution. Simply put: one component of solution is thermopholic, while the other is thermophobic. Thus, thermopholic component tends to diffuse towards higher temperature regions while the opposite is true for thermophobic component. In an attempt to describe it mathematically, several mathematical equations have been proposed. The simple yet useful form has a diffusion coefficient dedicated to just thermophoresis for numerical studies, and it is proportional to $c(1-c)$. This means that, the more different entities interact by intermolecular force, the more intense the thermophoresis is. This is seen in several numerical studies [30] [29] [31].

The idea of polymerization gradient is more theoretical because, in experimental setting, there are several mechanisms that complicate the process, altering the properties of the polymers. For example, suppose the degree to which polymers photo-cross-link depends on the intensity of the light. Then, when the gradient of light intensity is established and applied to solution, one would expect that the degree of cross-linking would vary across the domain. However, there are several factors that complicate the processes, such as the entanglements of the polymers and solution. In this study, ideal case is assumed, which is useful for isolating the effect of one parameters from other complications. To achieve this, Flory Huggins equation, which account for the polymerization level, is used.

The idea of carrying out the spinodal decomposition after establishing concentration gradient has been observed. Concentration gradient can be established by evaporation. As can be seen later, if the solution that is not uniformly mixed is quenched, structures that already exist direct the establishment of new morphology. Thus, different regions within the solution may result in different structures eventually. 


\subsection{Wetting}

The boundaries between the solution and surfaces is important in phase separation. As mentioned earlier, this boundary condition is required for the solution to Cahn-Hilliard to be unique. The surface potential directs the solution in by twisting the gradient of the solution immediately next to surface. This ability of the solution to maintain certain types of contact with the surface is wetting. This means that, the first derivative of the solution normal to the surface is dictated by certain values. The surface may have preferential attractions to either component of the solution. Typically, in numerical studies that do not concern with surface attraction and non-periodic boundary condition is used, the dot product of concentration gradient and unit vector normal to the surface is set to zero to indicate that there is no preferential attraction, as shown in equation (3.2.7). However, there have been experimental and numerical studies when surface potential is apparent. As will be discussed later, the configuration of the solution prior to quenching influences the course of phase separation in the early stage significantly, thus if surface potential is indeed present, it should not be ignored. In numerical studies, it is applied as the boundary conditions of the first derivative of concentration with respect to normal of the surface. Schmidt and Binder [70] have suggested numerical model to study this phenomenon. For numerical convenience, it is often a linear function of concentration at the boundaries. The surface potential can be easily manipulated, and can create several different patterns. Some patterns have been mentioned in previous section: lamellar [36], check-board pattern on the side view by inducing wetting with stripe patterns [38], and circular wave [40]. The similar observation can be repeated with present model and are shown in Figure B - 1 as Cases 3.B, 3.C, and 3.D respectively. For detailed parameters, please refer to Table B -1. Case 3.A is included as a control as it has no wetting.

\subsection{Noise}

The noise plays crucial role in phase separation of multi-phase polymer solution. In fact, most of the spinodal decomposition that lead to random pattern is dictated by the randomness of the 
noise, as any small fluctuation spontaneously leads to phase separation if the solution is unstable. The effect of the noise is especially significant when the solution is closer to uniformly mixed state. For metastable solution, it is important as the source of nucleation. If the noise, by some statistical chance, generates a configuration whose phase separation leads to lower energy, then nucleation and growth occurs. Cook has suggested that the noise term should be accompanied together with Cahn-Hilliard's theory, thus it is often referred to as Cahn-HilliardCook equation. Its dynamics is as following:

$$
\frac{\partial c}{\partial t}=\nabla \cdot\left[M \nabla\left(\frac{\partial f}{\partial c}-2 \kappa \nabla^{2} c\right)\right]+\eta
$$

Where $\eta$ is the noise. The noise may arise as random thermal fluctuation within the solution. The nature of the noise is usually studied from statistical perspectives. For this study, however, the noise term is included only in the initial configuration as the ignitions for spinodal decomposition. The noise term is otherwise omitted throughout the simulation.

\subsection{Some notable theories}

Ever since Cahn-Hilliard model and Flory-Huggin' theory were proposed, there have been publications that serve to better the model. Here, in this last section of Chapter 3 , only some of the most important ones are mentioned.

Nobel Prize laureate, Pierre-Gilles de Gennes [71], has helped to modify Flory-Huggins equation by accounting for the immediate environment, or gradient. Because the equation is based on linear assumption, it is omitted in this thesis as it slows down the time step too much, especially in later stage. Dynamics of metastable solution has been described formally in 1-d by Bates and Xun [72] [73] [74] from mathematical perspective. 


\section{Chapter 4 Mathematical modeling}

In this chapter, the mathematical methods of approaching the problems specified earlier are briefly discussed. Because there are many problems to be discussed in this thesis, some of the derivations and technical details are included in the appendix for neatness. The dynamics of diffusion of binary polymer is described using theory of Cahn-Hilliard alone.

\subsection{Galerkin finite element method (GFEM)}

The dynamics of binary polymer solution obeys Cahn-Hilliard equation with Flory-Huggin's equation incorporated and also appropriate boundary conditions. To study this behavior from engineering perspective, the governing equation is solved numerically. Here, Galerking finite element method is used. The appropriate mesh with multi elements is generated for chosen domains. The weights at each node is computed for every time step by using NewtonRaphson's method. In this method, each node carries weight(s) which stores the information of the configuration. The weights at each node only carries the information in adjacent element(s) only. This way, at any point in a domain, which must belong to some element, the weight is computed from the weights information stored within all corners, i.e.

$$
c=\sum_{i}^{N} c_{i} \varphi_{i}=\sum_{e} \sum_{\tau} \sum_{\omega} c_{\tau}^{\omega} \varphi_{e} \varphi_{\tau}^{\omega}=\left.\left(\sum_{\tau} \sum_{\omega} c_{\tau_{e}}^{\omega} \varphi_{\tau_{e}}^{\omega}\right)\right|_{\begin{array}{c}
e_{e=\text { element }} \\
\text { containing } \\
\text { the point } \\
\text { of interest }
\end{array}}
$$

Where $c_{\tau}^{\omega} \in \mathbb{R}$ is the weights at specific node, $\omega$ identifies the corner, $\tau$ is the type of information stored in the node, $\varphi_{\tau}^{\omega}$ is the global basis functions, $e$ is the element's identity, $N$ is 
the number of weights. For more details, please refer to Appendix $\mathrm{A} . c_{\tau}^{\omega}$ is the values that are adjusted by Newton-Raphson's method for multivariable to determine all constants. It is done by obtaining residual vector and Jacobian matrix. For example, for a given problem:

$$
h=g\left(c_{1}, c_{2}, c_{3}, \ldots\right)
$$

Each entry in the residual vector is given by:

$$
R_{i}=\int_{V}\left[h-g\left(c_{1}, c_{2}, c_{3}, \ldots\right)\right] \varphi_{i} \partial v
$$

And each entry of Jacobian matrix is given by:

$$
J_{i j}=\frac{\partial R_{i}}{\partial c_{i}}
$$

Where $i$ and $j$ are the number of row and column, respectively.

The adjustment for every Newton-Raphson for every node can be computed:

$$
\left(\begin{array}{c}
c_{1_{n+1}} \\
c_{1_{n+1}} \\
\vdots \\
c_{N_{n+1}}
\end{array}\right)=\left(\begin{array}{c}
c_{1_{n}} \\
c_{1_{n}} \\
\vdots \\
c_{N_{n}}
\end{array}\right)-J^{-1} R
$$

The governing equation has fourth order. As it can be seen later, the model can be used to reduce the problem into second order equation. Since the equation to be implemented still involves second derivative in each component, bicubic Hermitian basis functions are selected, which allows weights at each node to account for the first derivative in each component, and also the mixed derivatives with respect to all components for basis functions that are not serendipity. For more details on the basis functions, please refer to Appendix A.

\subsection{Governing equations and implementation}

\subsubsection{Governing equations, assumptions, and non-dimensionlizations}

Recall that, from previous chapter, the dynamics of multi-phase polymer as described by CahnHilliard: 


$$
\frac{\partial c}{\partial t}=\nabla \cdot\left[M \nabla\left(\frac{\partial f}{\partial c}-2 \kappa \nabla^{2} c\right)\right]
$$

For a closed system, there must be no mass-flux at the boundary. Furthermore, zero surface potential of the solution is assumed.

$$
\begin{aligned}
& \vec{J} \cdot \hat{n}=0 \text { on } \partial \Omega \\
& \nabla c \cdot \hat{n}=0 \text { on } \partial \Omega
\end{aligned}
$$

Flory-Huggins' equation is as shown in previous chapter.

$$
\frac{\partial f}{\partial c}=\frac{k_{b} T}{v}\left(\frac{\ln (c)}{n_{1}}-\frac{\ln (1-c)}{n_{2}}+\frac{1}{n_{1}}-\frac{1}{n_{2}}+\frac{\chi}{n_{1}}(1-2 c)\right)
$$

For this thesis, the mobility, $M$, and the interaction parameter, $\kappa$ are assumed constant. Such assumptions do not hinder results in this thesis unreliable. With these assumptions in mind, governing equation can now be rewritten in dimensionless form. Here, it is time to also introduce a dimensionless term to account for thermophoresis. Suppose $D_{T}$ is the dimensionless diffusion coefficient which is independent of position, is driven by thermophoresis and is independent of Flory-Huggins' mechanism, it is commonly expressed as the following [29] [31] [30]:

$$
\frac{\partial c^{*}}{\partial t^{*}}=\nabla^{* 2}\left(D^{*} T^{*} \frac{\partial f^{*}}{\partial c^{*}}-\nabla^{* 2} c^{*}\right)+\nabla^{*} \cdot\left[D_{T} c^{*}\left(1-c^{*}\right) \nabla^{*} T^{*}\right]
$$

Where:

$$
\begin{gathered}
\frac{\partial f^{*}}{\partial c^{*}}=\frac{v}{k_{b} T} \frac{\partial f}{\partial c}=\frac{\ln \left(c^{*}\right)}{n_{1}}-\frac{\ln \left(1-c^{*}\right)}{n_{2}}+\frac{1}{n_{1}}-\frac{1}{n_{2}}+\frac{\chi^{*}}{n_{1}}\left(1-2 c^{*}\right) \\
c^{*}=c \\
T^{*}=\frac{T}{\theta} \\
\chi^{*}=\frac{1}{2}-\Psi\left(1-\frac{1}{T^{*}}\right)
\end{gathered}
$$




$$
\begin{gathered}
D^{*}=\frac{k_{b} L^{2} \theta}{2 \kappa v} \\
\nabla^{*}=L \nabla \\
t^{*}=\frac{2 \kappa M t}{L^{4}}
\end{gathered}
$$

$L$ is a length with appropriate unit. Asterisks, "*”, is used to indicate that the parameter has been non-dimensionalized. For this entire thesis, $\Psi=1$.

If $D_{T}>0$, component 1 is thermophilic, else if $D_{T}<0$, the solution is thermophobic. Note that Flory-Huggins term is also dependent on temperature gradient, so the net flow due to temperature will depend on both terms. Thermophoresis term only appears in general governing equation as seen in (4.2.5) and mass flux. Thus, some calculations such as the energy will neglect this term. For this entire thesis, $D_{T}$ is made zero for simplicity so there is no need to worry about this term for this thesis.

For the convenience of discussion, the energy, potential, mass flux, and Flory-Huggins equations are also non-dimensionalized as following:

$$
E^{*}=\frac{E}{\kappa L}=\int_{V^{*}}\left(2 D^{*} T^{*} f^{*}+\left|\nabla^{*} c^{*}\right|^{2}\right) \partial v^{*}
$$

Where:

$$
\begin{gathered}
V^{*}=V / L^{3} \\
\partial v^{*}=\frac{\partial v}{L^{3}}
\end{gathered}
$$

The potential difference in the solution is:

$$
\left(\mu_{2}^{*}-\mu_{1}^{*}\right)=\frac{L^{2}\left(\mu_{2}-\mu_{1}\right)}{2 \kappa}=D^{*} T^{*} \frac{\partial f^{*}}{\partial c^{*}}-\nabla^{* 2} c^{*}
$$

The mass flux is:

$$
\overrightarrow{J^{*}}=\frac{L^{3} \vec{J}}{2 \kappa M}=\nabla^{*}\left(D^{*} T^{*} \frac{\partial f^{*}}{\partial c^{*}}-\nabla^{* 2} c^{*}\right)+D_{T} c^{*}\left(1-c^{*}\right) \nabla^{*} T^{*}
$$

The Flory-Huggins' equation is: 


$$
f^{*}=\frac{v f}{k_{b} T}=\left(\frac{c^{*}}{n_{1}} \ln c^{*}+\frac{1-c^{*}}{n_{2}} \ln \left(1-c^{*}\right)+\chi \frac{c^{*}\left(1-c^{*}\right)}{n_{1}}\right)
$$

For neatness, from this point onwards, asterisks are not shown. All parameters mentioned for the rest of the thesis, except for some sections in Appendix A, are dimensionless.

\subsubsection{Implementation}

In an attempt to implement GFEM on the governing equation mentioned, the residuals and Jacobian matrix need to be fully derived. The derivations should be done in such way that the computational cost is minimal while keeping all the features of the interest (e.g. the presence of temperature gradients, gradient of polymerization, etc.). Minimizing computational cost means that the equations to be implemented for each element of residual and Jacobian matrix must be as short as possible. While nonlinearity of Cahn-Hilliard equation is accounted, free energy term needs to be not expanded too much. This is possible by taking advantage of no-flux boundary condition. Recall that:

$$
\vec{J} \cdot \hat{n}=0 \text { on } \partial \Omega
$$

By divergence theorem:

$$
\begin{aligned}
\int_{V}(\nabla \cdot \vec{J}) \varphi_{i} \partial v & =\int_{V} \vec{J} \cdot \nabla \varphi_{i} \partial v-\int_{V} \nabla \cdot\left(\varphi_{i} \vec{J}\right) \partial v \\
& =\int_{V} \vec{J} \cdot \nabla \varphi_{i} \partial v-\left.\oint_{V} \varphi_{i}(\vec{J} \cdot \hat{n})\right|_{\partial \Omega} \partial \tau=\int_{V} \vec{J} \cdot \nabla \varphi_{i} \partial v
\end{aligned}
$$

Ultimately:

$$
R_{i}=\int_{V} \frac{\partial c}{\partial t} \varphi_{i} \partial v+\int_{V} \vec{J} \cdot \nabla \varphi_{i} \partial v
$$


Thus, order of the entire mass flux term has been reduced.

$$
\begin{aligned}
R_{i}=\int_{V} \frac{\partial c}{\partial t} \varphi_{i} \partial v & \\
& +D \int_{V}\left[\left(\frac{\partial f}{\partial c}+T \frac{\partial^{2} f}{\partial c \partial T}\right) \nabla T+T \frac{\partial^{2} f}{\partial c^{2}} \nabla c+T \frac{\partial^{2} f}{\partial c \partial n_{2}} \nabla n_{2}\right] \\
& \cdot\left(\nabla \varphi_{i}\right) \partial v+D_{T} \int_{V} c(1-c)\left(\nabla T \cdot \nabla \varphi_{i}\right) \partial v+\int_{V} \nabla^{2} c \nabla^{2} \varphi_{i} \partial v \\
& +\left.\oint_{V} \nabla^{2} c\left(\nabla \varphi_{i} \cdot \hat{n}\right)\right|_{\partial \Omega} \partial \tau
\end{aligned}
$$

Where:

$$
\begin{gathered}
\frac{\partial f}{\partial c}=\frac{\ln (c)}{n_{1}}-\frac{\ln (1-c)}{n_{2}}+\frac{1}{n_{1}}-\frac{1}{n_{2}}+\frac{\chi}{n_{1}}(1-2 c) \\
\frac{\partial^{2} f}{\partial c^{2}}=\frac{1}{c n_{1}}+\frac{1}{(1-c) n_{2}}-\frac{2 \chi}{n_{1}} \\
\frac{\partial^{2} f}{\partial c \partial T}=\frac{(1-2 c)}{n_{1}} \frac{\partial \chi}{\partial T} \\
\frac{\partial^{2} f}{\partial c \partial n_{2}}=\frac{1+\ln (1-c)}{n_{2}^{2}} \\
\frac{\partial \chi}{\partial T}=\frac{\Psi}{T^{2}}
\end{gathered}
$$

Conveniently, the term $\left.\oint_{V} \nabla\left(\varphi_{i} \nabla^{2} c \cdot \hat{n}\right)\right|_{\partial \Omega} \partial \tau$ needs not be evaluated [4] because $\nabla \varphi_{i}$ is zero at the boundaries of the weights functions. If the weight functions are located at the boundary of the domain, the weight functions with $\nabla \varphi_{i}$ not being equal to zero at the boundaries also need not be evaluated because boundary condition is applied to sparse matrix and residual vector corresponding to these. This is the advantage of Hermite basis functions for this particular model. It is approximated as zero for Hermite basis functions with serendipity for this thesis.

Here, $\frac{\partial c}{\partial t}$ in (4.2.16) is evaluated by assuming linear change in configuration, i.e. it is simply the change in nodal weight within the time step: 


$$
\frac{\partial c}{\partial t} \approx \frac{c_{n}-c_{n-1}}{\Delta t}
$$

Where $c_{n-1}$ and $c_{n}$ are previous and current concentration, respectively, and $\Delta t$ is the time step. This assumption will fail if the time step is too large because the solution is harder to predict. Because the prediction must be reasonably correct, the time step is made smaller especially in late stages of spinodal decomposition.

Each entry for Jacobian matrix is the derivative of $R_{i}$ with respect to nodal weights. Every time step, because the configuration in previous time step has been defined and is therefore constant, by (4.2.25):

$$
\frac{\partial}{\partial c_{j}} \frac{\partial c}{\partial t} \approx \frac{\varphi_{j}}{\Delta t}
$$

Now, each entry of Jacobian matrix can be readily evaluated:

$$
\begin{aligned}
J_{i j}=\frac{\partial R_{i}}{\partial c_{j}}=\int_{V} & \frac{\varphi_{i} \varphi_{j}}{\Delta t} \partial v \\
& +D \int_{V} \varphi_{j}\left[\left(\frac{\partial^{2} f}{\partial c^{2}}+T \frac{\partial^{3} f}{\partial c^{2} \partial T}\right) \nabla T+T \frac{\partial^{3} f}{\partial c^{3}} \nabla c\right. \\
& \left.+T \frac{\partial^{3} f}{\partial c^{2} \partial n_{2}} \nabla n_{2}\right] \cdot\left(\nabla \varphi_{i}\right) \partial v+D \int_{V} T \frac{\partial^{2} f}{\partial c^{2}}\left(\nabla \varphi_{i} \cdot \nabla \varphi_{j}\right) \partial v \\
& +D_{T} \int_{V} \varphi_{j}(1-2 c)\left(\nabla T \cdot \nabla \varphi_{i}\right) \partial v+\int_{V} \nabla^{2} \varphi_{i} \nabla^{2} \varphi_{j} \partial v
\end{aligned}
$$

Where:

$$
\begin{gathered}
\frac{\partial^{3} f}{\partial c^{3}}=\frac{-1}{c^{2} n_{1}}+\frac{1}{(1-c)^{2} n_{2}} \\
\frac{\partial^{3} f}{\partial c^{2} \partial T}=-\frac{2}{n_{1}} \frac{\partial \chi}{\partial T} \\
\frac{\partial^{3} f}{\partial c^{2} \partial n_{2}}=\frac{-1}{(1-c) n_{2}^{2}}
\end{gathered}
$$


Thus, governing equations throughout the entire the thesis have been established. For this entire thesis, $n_{1}=1$ at all time. By noting non-dimensionalized equations (4.2.5) and (4.2.6), the overall size of solvent molecule can be accounted for in diffusion coefficient, $D$. Thus, $n_{2}$ really means the ratio of the size between solvent and polymer.

Finally, another crucial boundary condition for wetting needs to be applied:

$$
\left.\nabla c\right|_{\partial \Omega} \cdot \hat{n}=g c+h
$$

This is done by taking advantage of GFEM and is the very reason bicubic Hermitian basis functions, with or without serendipity, is chosen. Before Newton-Raphson iterations are carried out, $\left.\nabla c\right|_{\partial \Omega}$ is computed manually everywhere using (4.2.34), then these values are manually assigned to corresponding weights. By observing (4.1.5), one can realize that, the weights corresponding to the derivatives normal to the surface should be varied by $g$ times the change of the concentration, thus $g$ is inserted into $J_{i j}$ where $i$ identifies the weight of interest and $j$ identifies the concentration there. Also, $J_{i i}$ should be set to one and the remainder of the row is set to zero, while $h$ is inserted into residual vector. This would cause complications for mixed term (the weights corresponding to multiple derivatives) if $g$ is not zero, so for wetting analysis, bicubic Hermitian basis functions with serendipity (See Appendix A) is used. Otherwise, if $g=0$, bicubic Hermitian basis functions without serendipity will work as mixed term is always set to zero because the derivative with respect to any component is a constant, which makes second derivative with respect to another orthogonal component zero.

\subsection{On algorithm featuring parallel computation and SuiteSparse}

The efficiency of numerical algorithm has been significantly improved. In this research, efforts were made to make numerical simulation as fast as possible, while resource is limited to standard home PC at the time this thesis was written. General specifications are: Intel ${ }^{\circledR}$ Core $^{\mathrm{TM}}$ i7-8700 CPU, 16GB 2666MHz RAM. To minimize computational cost, the results of repetitive computations were stored in RAM as appropriate. Storing all variables that do not change 
throughout the simulation in RAM and passing them into functions does not always make simulations faster especially if the argument is too large and computation is simple. Thus, deliberate adjustments on algorithms is done by trial-and-error's method to see which algorithm has less running time. Nowadays, CPU with up to 6 cores, like the one used to run all simulations in this thesis, is readily available on mainstream computer. Thus, parallel computation, in which all cores are run at the same time for quicker computation, is in more demand. Parallel computation algorithm is implemented in the code. To achieve this, every non-zero entry in large multi-dimension matrices such as Jacobian matrix is computed independently from one another, i.e. they are not computed as one large loop like before. In order to minimize communication between the cores, the entries of large arrays are grouped into a number of sets equal to number of cores and specify which set of computations each core is responsible for. Even with better CPU, RAM was still limited to $16 \mathrm{~GB}$. To reduce the memory, only non-zero element in Jacobian matrix is stored with identity indexes. Note that, this numerical method involves a large matrix whose most entries are zero (aka sparse matrix). For example, in this thesis, $2-d$ mesh of size $256 \times 256$ is used for bicubic Hermitian basis functions, which requires Jacobian matrix of the size $262,144 \times 262,144$, which means it has $68,719,476,736$ entries in total, of which only 9,388,096 entries are non-zero before boundary condition is applied and therefore needs to be saved. The reduction is even more profound in 3-d mesh. Saving only non-zero entries has significantly reduced the amount of RAM required for simulation, thus now the author can work with larger mesh with less chance of crashing. MATLAB R2018a is perfect for this research as it has built-in tool, sparse function, to handle it. Tremendous credit must be given to SuitSparse [5] package, which is the best package, to the best of author's knowledge, for handling large sparse matrix division. It has been featured in many applications such as Google Map. MATLAB R2018a has also made algorithm readily callable, significantly reducing simulation time. By working with this high-level programming language, it puts the author at the ease of coding and provided the best tools to reduce computation time from days to just few minutes for similar simulations intended for same purpose but with different algorithms. Reducing computational time is important for this thesis because, on many occasions, the solution is put into late stage, which is slow in computation because adaptive time step is made smaller because of changing behavior of the solution. Here, 
adaptive time-step is adjusted as necessary throughout the entire simulation because NewtonRaphson method requires that the first guess of the solution must be appropriately correct to certain extent, otherwise the solution does not converge. Small time step makes simulation too slow, while large time step makes the first guess of Newton-Raphson' iteration harder to predict appropriately. Thus, if the number of Newton-Raphson's iterations is small, the time step is made larger. If the solution diverges or there are too many Newton-Raphson's iterations, the time step is made smaller. Lastly, for this entire thesis, the author deliberately picked the simulation range that will help the concentration, $c$, to not get too close to 0 or 1 to avoid numerical error, especially after early stage. This can be done by carefully considering two equilibrium values, $e_{1}$ and $e_{2}$, from phase diagram.

\subsection{Fourier transform and Inverse Fourier transform}

Fourier transform is carried out for analytical purpose throughout the entire thesis. The concentration, $c$, is discretized into nodes in the $M \times N$ mesh. The Fourier transform for 2-d is computed as following:

$$
\begin{aligned}
\hat{\hat{c}}\left(k_{1}, k_{2}\right)=\frac{1}{\sqrt{M N}} \sum_{m=1}^{M} \sum_{n=1}^{N}\left[c(m, n)-c_{a v e}\right] e^{-2 \pi i\left[\frac{k_{1}}{M}(m-1)+\frac{k_{2}}{N}(n-1)\right]} \\
=\frac{1}{\sqrt{M N}} \sum_{m=1}^{M} \sum_{n=1}^{N}\left\langlec ( m , n ) \left\{\operatorname { c o s } \left[\frac{2 \pi k_{1}}{M}(m-1)\right.\right.\right. \\
\left.\left.\left.+\frac{2 \pi k_{2}}{N}(n-1)\right]-i \sin \left[\frac{2 \pi k_{1}}{M}(m-1)+\frac{2 \pi k_{2}}{N}(n-1)\right]\right\}\right\rangle
\end{aligned}
$$

Where $k_{1}$ and $k_{2}$ are the frequencies in each component, $m$ and $n$ are indexes of row and column of discretized mesh. Note that, equation (4.4.1) computes Fourier transform of relative concentration (i.e. the difference between concentration and average concentration) rather than absolute concentration. This way, $\hat{\hat{c}}\left(k_{1}=0, k_{2}=0\right)=0$ at all-time and makes it easier to visualize amplitude spectrum, as $\hat{\hat{c}}\left(k_{1}=0, k_{2}=0\right)$ would go to infinity, if absolute 
concentration is used (Addition of Fourier transform of average concentration gives Dirac delta function.). Analysis on Fourier spectrum is especially useful because, spinodal decomposition from uniformly mixed state usually yields characteristic frequency in early stage, as shown in section A.1. By comparing the values obtained from equation (4.4.1) and equation (A.1.22), it is possible to verify the correctness of simple spinodal decomposition and follows the difference in other cases. Also, it is useful for distinguishing high and low frequency structures separately. For example, as will be shown in Chapter 5.1, double quench may lead to two distinct structures with different frequency. In an attempt to further distinguish them visually, bandpass filter is applied is some cases. For these instances, Fourier transform is carried out using $\mathrm{ftt}$ function bult into MATLAB R2018a to minimize the error in the magnitude upon inversion, which is done by calling ifft. The band-pass filter is applied by zeroing all amplitude or real and imaginary components the Fourier transformed data that are outside the filter, then it is converted back with inverse Fourier transform. The filters featured for this thesis is often has a ring shape for 2-d, which often spans the area between the circle of radius $r_{1}$ and another circle of radius $r_{2}$. In mathematical symbol, it means:

$$
\begin{gathered}
\hat{\hat{c}}\left(k_{1}, k_{2}\right)=0 \text { if } \sqrt{{k_{1}{ }^{2}+{k_{2}}^{2}}^{2}}<r_{1} \text { or } \sqrt{{k_{1}{ }^{2}+{k_{2}}^{2}}^{2}}>r_{2} \\
\hat{\hat{c}}\left(k_{1}, k_{2}\right)=\mathcal{F}[c] \text { otherwise }
\end{gathered}
$$

The notation, $\left(r_{1}, r_{2}\right)$, is used to specify the band-pass filter used. 


\section{Chapter 5 Results and Discussions}

This chapter discusses some of the important results obtained from this thesis. Sometimes, preliminary results are repeated to highlight some of the notable features that are useful for comparisons and discussions. In this thesis, mainly metastable phase separation in the solution without nucleation from the noise is investigated, since the noise involves more statistical physics. In order to initiate phase separation, several methods are used:

1. Double quench: Solution is first quenched into unstable region then temperature or polymerization is changed so that the solution is in metastable region.

2. Anisotropic quench: Temperature or polymerization gradients are established so that part of the solution is in metastable region, while other part is in unstable region.

3. Wetting: Boundary, or domain wall, is made to have preferential affinity to one of the components. The solution within such cell is quenched into metastable region.

4. Concentration gradient: Concentration gradient is generated so that the solution is in unstable region locally, while the average concentration is still kept at metastable region.

Using those methods mentioned above is more strategic and useful from engineering point of view. Aside from metastable quench in the solution, some of the patterns that are similar to some experimental observations are shown. This helps to add plausible explanation for some experimental observations using just present model, without the complications from other factors such as elasticity.

Lastly, a section is dedicated to demonstrate the capability of present numerical method to be performed on curved boundaries, hence any complex geometry with non-periodic boundary 
conditions. It means that, study of surface-directed phase separation of multi-phase solution in any complex geometry is now possible with any standard home PC.

Parameters for all simulations are summarized in Table B - 1, included in Appendix B.

\subsection{Double quench}

\subsubsection{Unstable first quench and unstable second quench: Some remarkable patterns}

While present results regarding double quenching by varying only temperature can be closely verified in experimental setting, double quenching by polymerization, cross-linking, or cracking may cause more complications. Because, during this process, the solution may locally vary in the ability to diffuse. The causes may include polymer entanglements, volume shrinkage/enlargement, and the variations in how the polymers undergo chemical changes. These events are random and should be studied as a statistical estimation. Thus, this part may be more theoretical than double quenching using temperature variation. In future model, it is highly suggested to include elastic potential energy term. Nonetheless, it can help to replicate some of the experimental results with some limitations.

Given that the structural morphologies, droplets or interconnected or others, will depend on how close the average concentration is away from critical concentration, since double quench by varying polymerization level will shift the critical concentration, one may expect to obtain more variety of morphologies by double quenching with polymerization. For example, in the experiment carried out by Tran Cong-Miyata' et al [75] [76] [19], P(S-stat-CMS)/PVME blend was cross-linked by labeling $\mathrm{P}(\mathrm{S}$-stat-CMS) with anthracenes, which can undergo photodimerization. When crosslinking carried out at a certain temperature between binodal line before photo-cross-linking and glass temperature, the binary polymer blend manifests concentric pattern morphology. In an attempt to replicate the phenomenon numerically, Furukawa [20] has demonstrated that, upon crossing metastable region, the blend undergoes nucleation and growth mechanism. As cross-linking proceeds to place the mixture in unstable region, the nuclei created will then act as the back bone and direct the solution to undergo 
spinodal decomposition in such way that phase separation proceeds around the nuclei, creating concentric pattern. The later publication by Okada, Masunaga and Furukawa [21], they argued that nucleation and growth event is an unlikely event because of high viscosity. This time, instead, the mobility and the skewness of phase diagram, which is related to polymerization, is varied with time. He especially paid attention to the mobility where several set of parameters yields a variety of concentric patterns. In this section, the author shall also demonstrate how the concentric pattern can be obtained. The difference is that, concentric pattern is obtained solely by double quenching, with different polymerization level while mobility is kept constant. Furthermore, a well-recognized Flory-Huggins's theory is used for this purpose for the first time. But varying the initial configurations, a variety of unique morphology not limited to concentric pattern can be obtained.

First quench is carried out at $D=50000, c_{\text {ave }}=0.690983006, T=0.56, n_{1}=1, n_{2}=3$ (Case 5.1.1.A) and is shown in Figure B - 1. This concentration is critical for the blend with $n_{1}=$ $1, n_{2}=5$. This proceeds typically with expected characteristic frequency $f_{c}=4.020059233$ and the beginning of the intermediate stage takes place at approximately $t=2.964788 \times$ $10^{-5}$. In this section, different configurations obtained at different iterations is used for double quench with only different polymerization, $n_{2}=5$, and all other parameters constant.

Figure 5.1-1 shows the second quench (Case 5.1.1.B) with configuration taken at $t=$ 1.411752 $\times 10^{-5}$ of the first quench (Case 5.1.1.A), when the phases have not separated significantly yet. Note that, for this section, author has included extra noise at $t=0$ because characteristic frequency in the second quench is higher, but the structure factor at these frequencies have been eliminated in the first quench. By adding the noise, which is a small random fluctuation in concentration at the beginning, it better allows the structure to adopt to favorable morphology. In Figure 5.1-2, at $t=1.057190 \times 10^{-7}$, the destruction in large droplets, which are breaking into smaller ones gradually, can be observed. The characteristic frequency of the solution at the second quench is calculated to be $f_{c}=13.01260336$, by equation A.1.22. Indeed, the frequency diagram in Figure 5.1-3 and Figure 5.1-4 reveal that there is a rise in the amplitude at this frequency (In this thesis, amplitude is the absolute squared of Fourier transformed configuration). Already-established structure factor at $f_{c}=$ 
4.020059233 is also growing, but it is not as fast. The beginning of the intermediate stage is seen at $t=2.572599 \times 10^{-7}$, where the amplitude at $f_{c}=13.01260336$ has dominated the entire morphology. As it enters late stage, step-by-step shift of dominant structure factor towards lower frequencies can be observed. These are typical phenomenon when the second quench is a lot deeper. Since it is at the critical concentration, the new morphology conforms an interconnected structures. Until the beginning of the intermediate stage, smaller droplets that have just appeared seem to connect with one another to form interconnected structures, while the late stage continue to show the same phenomenon but also the disappearance in the smaller structures and enlarging of the neighboring structures. Figure 5.1-5 shows the maximum amplitude of logged structure factor over time. The 'kink' at about $t=1.4 \times 10^{-7}$ is the time when structure of newly emerging structures surpasses that of previously dominating structures. Relative energy of this simulation is shown in Figure 5.1-6. For this thesis, relative energy is defined as the difference between dimensionless energy of the solution and that of the solution when it is perfectly uniformly mixed. The relative energy drops quickly starting about $t=2.2 \times 10^{-7}$. This is after the newly established structures have become dominant and well-defined. The phase separation thus occurs quickly as smaller droplets of higher frequency. Before this fast phase separation period, there is a period in which the relative energy does not seem to vary much. This is the lag time in which the solution re-arranges itself to adopt to new environment by conforming to thermodynamically favorable morphology.

Next, the configuration at 117th iteration, or $t=2.682418 \times 10^{-5}$, from the first quench (Case 5.1.1.A) is taken, then is subject to second quench with the same parameters as above (Case 5.1.1.C). The overall process is shown in Figure 5.1-7. At this configuration, the characteristic frequency from the first quench has already been well established, with $\|F\|^{2} \approx 0.71$ at $f_{c}=$ 4.0, as shown in Figure 5.1-10. This peak continues to rise in second quench, but at a rate relatively slower than some other peaks. Here, the rise in structure factor at about $f=13.0$ is observed, which is close to new characteristic frequency and is typical of deeper quench. Remarkably, there are broad peaks arising in between $f=6$ and $f=12$. Ultimately, in Figure 5.1-8, a concentric pattern has resulted over time. These broad peaks in the intermediate frequency range actually rise fastest. In Figure 5.1-13, there is a "kink" at about $t=1.65 \times$ 
$10^{-5}$. This is when the amplitude of the broad peaks mentioned surpassed that of the initially established structure factor at $f_{c}=4.0$. All peaks seem to be approaching stationary state over time since the slope is positive decreasing. In an attempt to understand these frequencies, band-pass filters are applied to analyze each frequency qualitatively. Figure 5.1-11 shows relative concentrations after band-pass filters are applied to configuration at $t=1.357740 \times$ $10^{-7}$. The initially established structure has been retained. They have low frequency and can be shown as $r<6$. High frequency noise, $r>14$, are relatively low except at the boundary. When comparing $\left(r_{1}, r_{2}\right)=(6,12)$ to $\left(r_{1}, r_{2}\right)=(12,14)$, the later has more co-continuous structure, while the former is less so. For $\left(r_{1}, r_{2}\right)=(12,14)$, there is relatively high value within the center of the macro spheres. Thus, this frequency range is responsible for any cavity within them. For $\left(r_{1}, r_{2}\right)=(6,12)$, they are more interconnected, or rather, seem to evolve around the low frequency macro-spheres, creating concentric ring around it. Within the ring arcs, high frequency co-continuous structures can be observed, as seen for $\left(r_{1}, r_{2}\right)=(12,14)$. Thus, it can be inferred that there are two competing processes here: one is where the ring with broad intermediate frequencies evolve around macro-spheres, adopting to initial macro-sphere's shapes, creating wave pattern starting from the edge of the macro-sphere then propagates outwards. Within it, independently, co-continuous structures with high characteristic frequency also evolves. Center of the macro-sphere is remarked by only this process. Figure 5.1-12 shows band-pass filters at $t=2.136128 \times 10^{-7}$. Band-pass filters are applied as following: First, the configuration of the solution is Fourier transformed into frequency domain using built-in fftn function in Matlab. All values outside the desired range are zeroed then the data is converted back to original domain using ifftn functions. The result is approximated by taking only the real values. The range are usually specified as a ring with inner radius of $r_{1}$ and outer radius of $r_{2}$, i.e. All values with $r_{1}<\sqrt{{k_{1}{ }^{2}+k_{2}{ }^{2}}_{2}}<r_{2}$ are kept, while all others are zeroed. Filter $\left(r_{1}, r_{2}\right)=$ $(12,14)$ shows that the contrast of characteristic frequency becomes higher within the ring arcs, while $\left(r_{1}, r_{2}\right)=(6,12)$ shows that the ring structures continue to become apparent even further. Figure 5.1-13 shows the maximum of logged structure factor of simulation discussed. The structure factor of low frequency structures keep evolving but decelerate in amplitude (as its concavity is down). At about $t=1.63 \times 10^{-7}$, the structure factor of newly emerging 
component becomes higher. Thus it takes longer for newly emerging structures to dominate than in Case 5.1.1.B. Figure 5.1-14 shows consistent drop in energy over time. This time, the drop in energy is less dependent on phase separation of newly emerging structures than in Case 5.1.1.B. The drop in energy is rather more consistent because the solution can no longer conform to the morphology with characteristic frequency because phase separation has already proceeded too far. It is thermodynamically better for the solution to retain the droplets from the first quench and phase separates in other regions, such as center of the droplets and the matrix. Thus, a period of lag-time is not observed like in Case 5.1.1.B. Figure 5.1-20 shows the maximum logged structure factor. Unlike previous 2 cases, 'kink' structure is absent at this time, thus the already established structures has been more dominant than in last 2 cases. Figure 5.1-21 confirms the steady drop in relative energy over time.

Finally, the configuration at 123 rd iteration, or $t=2.964788 \times 10^{-5}$. This is the beginning the intermediate stage in the first quench. Upon second quench (Case 5.1.1.D, Figure 5.1-15), cavities within both the droplets and the matrix emerge, as soon as $t=5.409373 \times 10^{-8}$. As the edges of the macro-spheres are raised higher in the concentration, the matrix attempts to lower in its concentration, creating sharp cavities between macro-spheres. The cavity within the matrix then forms the polygons around macro-spheres as shown at $t=1.294053 \times 10^{-7}$. This time, the edges of polygons lie in between about the midpoints between macro-spheres. Any points on the edges of the polygonal shapes are created about the midpoints of the closest macro-spheres, therefore the shapes. Most of the polygons are hexagons. This structure is quite like typical cellular network in plants, with the cell walls between them. As the simulation proceeds towards $t=1.946080 \times 10^{-7}$, the cavities within the droplets fade. The matrix also rises higher in the concentration, resulting in the disappearance of the "cell-wall" like structures in contour plot. This process can be more closely analyzed with structures factors. In Figure 5.1-17 and Figure 5.1-18, the initially established structure factor at characteristic frequency at about $f_{c}=4.0$ persisted throughout the entire simulation time frame and it also grows rather slowly compared to other notable ones. There is a rise broad band of peaks in the range of $f=$ 6 to $f=15$ at $t=1.294053 \times 10^{-7}$. Towards $t=1.946080 \times 10^{-7}$, the structure factor around $f=13$ is collapsing. This indicates the disappearance of the sharp cavities. To analyze 
which morphology different specific frequency ranges account for, band-pass filters at $t=$ $1.632703 \times 10^{-7}$ are applied and shown in Figure 5.1-19. Morphology of $\left(r_{1}, r_{2}\right)=(11,15)$ is quite continuous, while that of $\left(r_{1}, r_{2}\right)=(5,11)$ is less so. Structure of $\left(r_{1}, r_{2}\right)=(5,11)$ seems to be the results of the already established macro-spheres, i.e. the wave that propagates from the center of the macro-spheres, while the structure of $\left(r_{1}, r_{2}\right)=(11,15)$ is the characteristic morphology that arise within the arcs of the concentric rings. Also note that, this frequency range is largely responsible for the cavities at the centers of the macro-spheres. Thus, there are three competing processes just like before, but a little different: the already established structure factor continues to rise and dominate as a major structure factor. Structure factor in the intermediate frequency range rises as a result of the wave pattern that forms around the edge of each macro-sphere. In this unique case, these is only one wave between spheres. These waves form hexagon structures as they try to stay furthest away from each sphere. Within the arcs waves and at the center of the macro-spheres, new structure factor of the characteristic frequencies results as co-continuous structures. These structures collapse first later on.

In this section, upon deeper second quench with different degree of polymerization with the average concentration being critical, a variety of configuration can be obtained with different initial configuration. It is a competing process, in which the waves around macro-spheres propagates outwards, while the structure factor with new characteristic frequencies arise within the center of macro-spheres and within the arcs of the waves or just everywhere if initial configuration has not phase-separated significantly yet. This way, a completely new interconnecting structures, a concentric pattern, or a cell wall-like structures may form. Concentric patterns obtained in this section hopefully shed some light into experimental observation by Tran-Cong-Miyata et al [75] [76] [19]. Notable difference is that, they have carried out photo cross-linking continuously rather than double quench. Nonetheless, if the timing is right, some configuration over the experimental range may become more significant than the others and behave like multiple quenching. While only three distinct morphologies are shown here, even more different morphologies might result with different initial configuration. This is left as the suggestion for future research. 

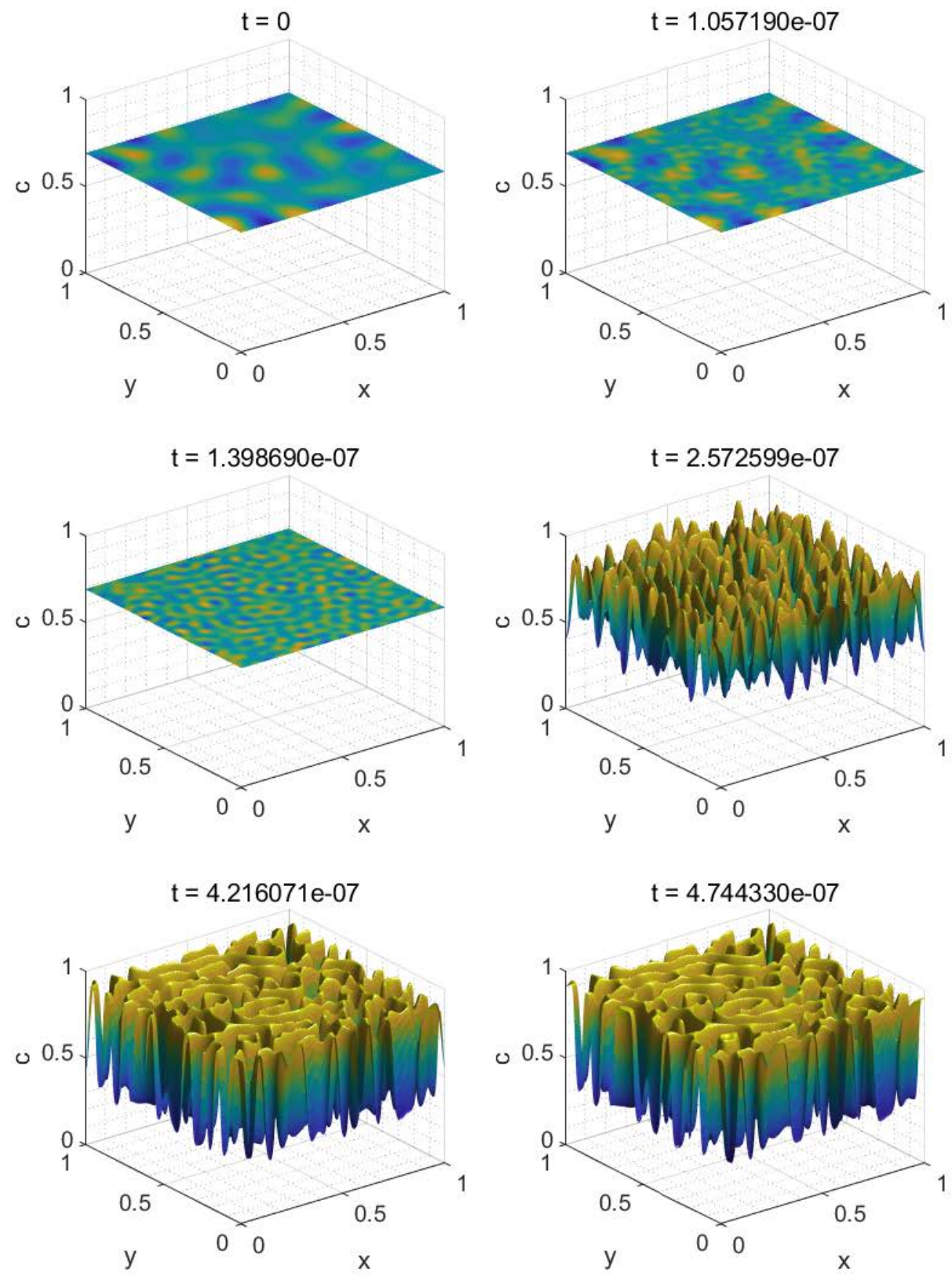

Figure 5.1-1 Concentration profile of second quench with initial configuration taken from first quench at $t=1.411752 \times 10^{-5}$ (Case 5.1.1.B) 

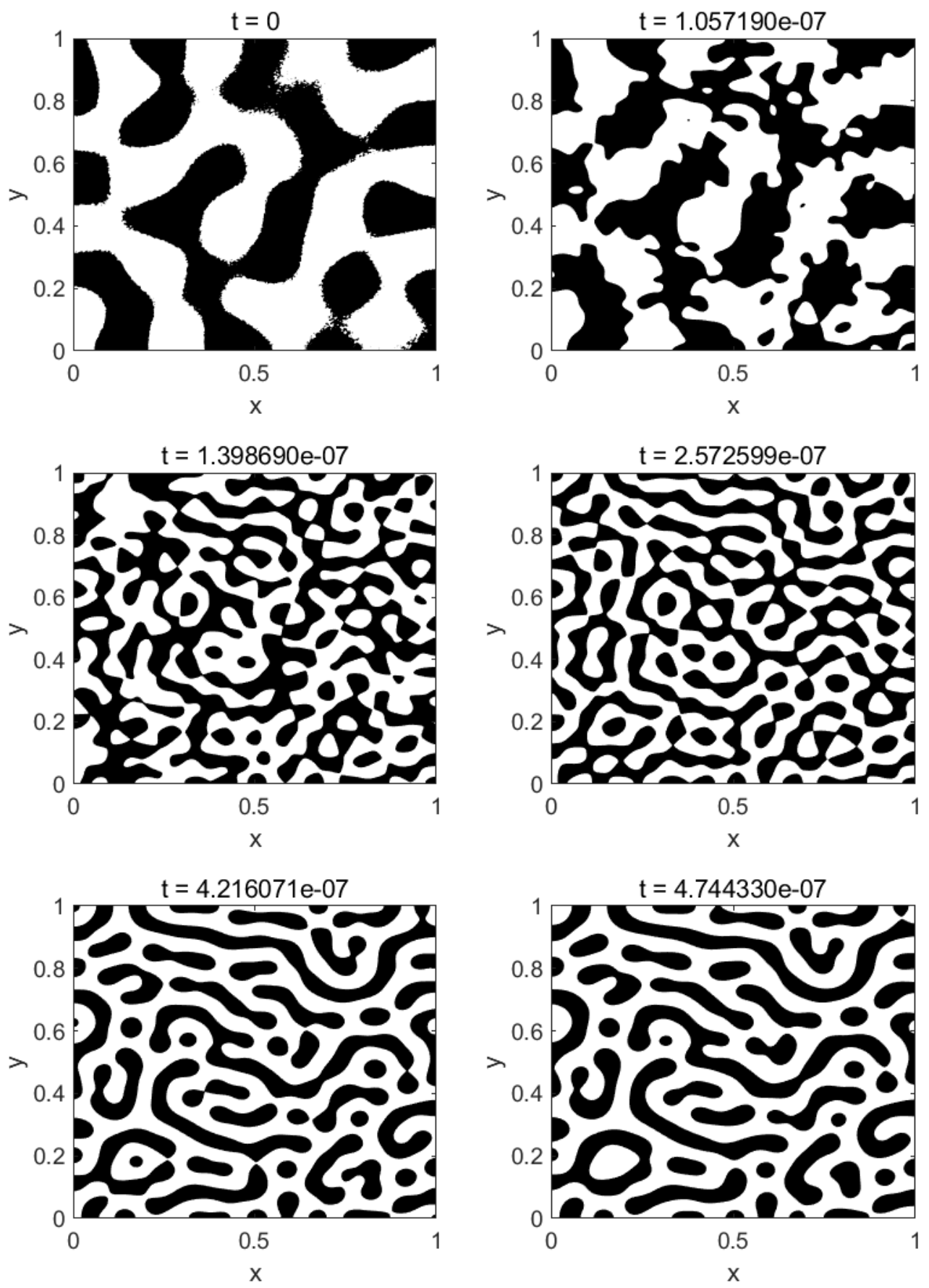

Figure 5.1-2 Contour plot of the concentration of the same simulation as in Figure 5.1-1 (Case 5.1.1.B) 

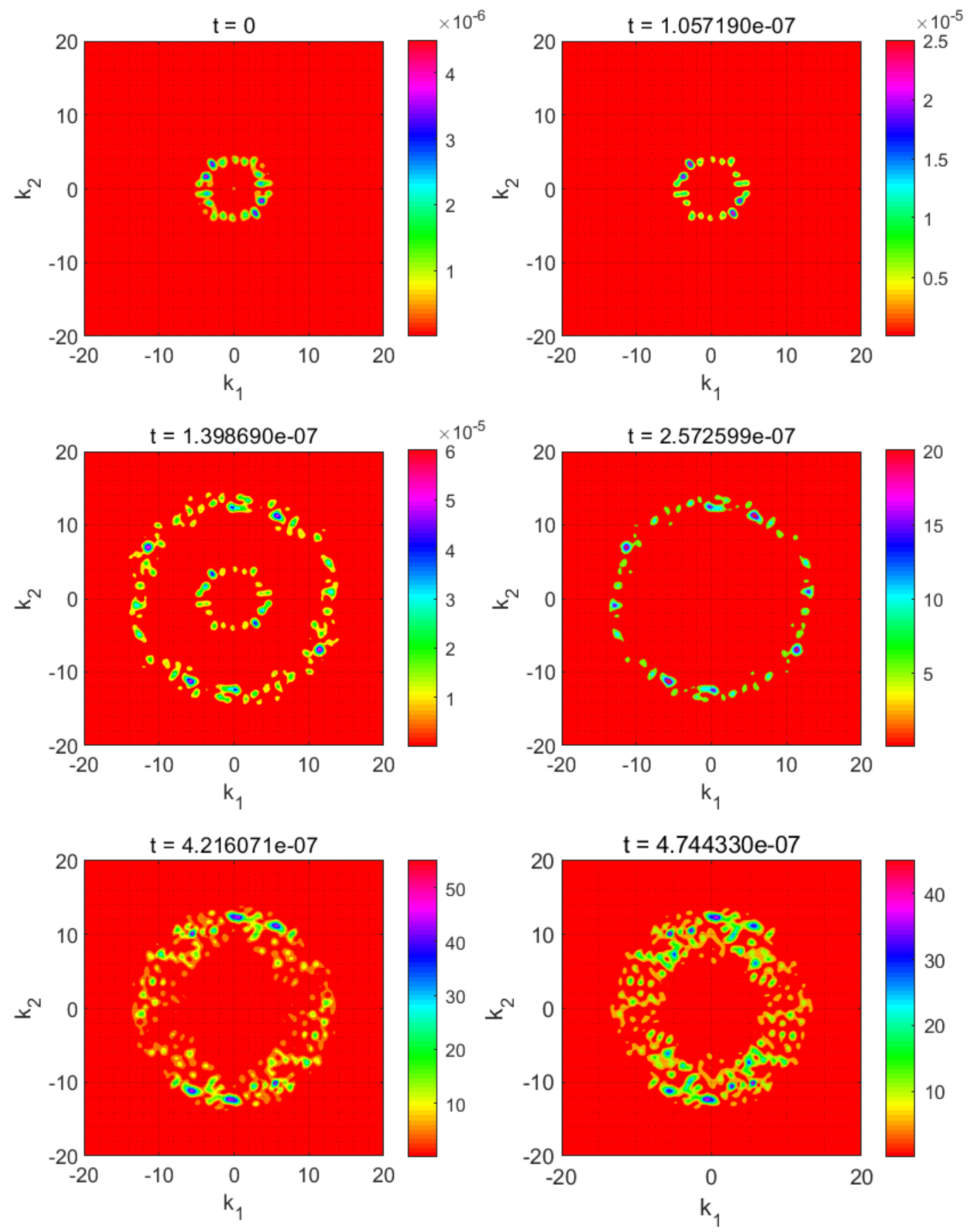

Figure 5.1-3 Amplitude spectrum of the same simulation as in Figure 5.1-1 (Case 5.1.1.B) 

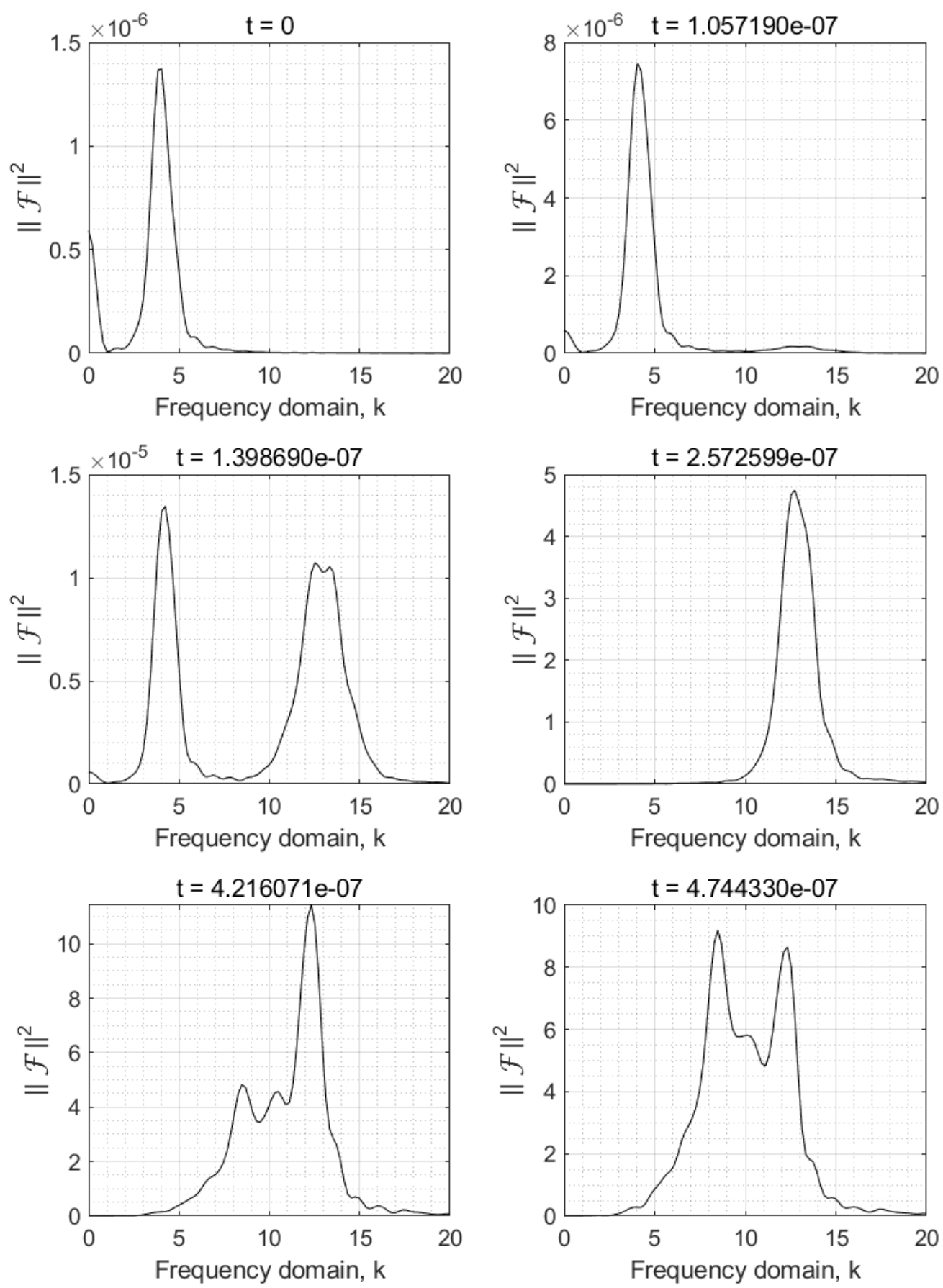

Figure 5.1-4 Circularly averaged amplitude spectrum of the same simulation as in Figure 5.1-1 (Case 5.1.1.B) 


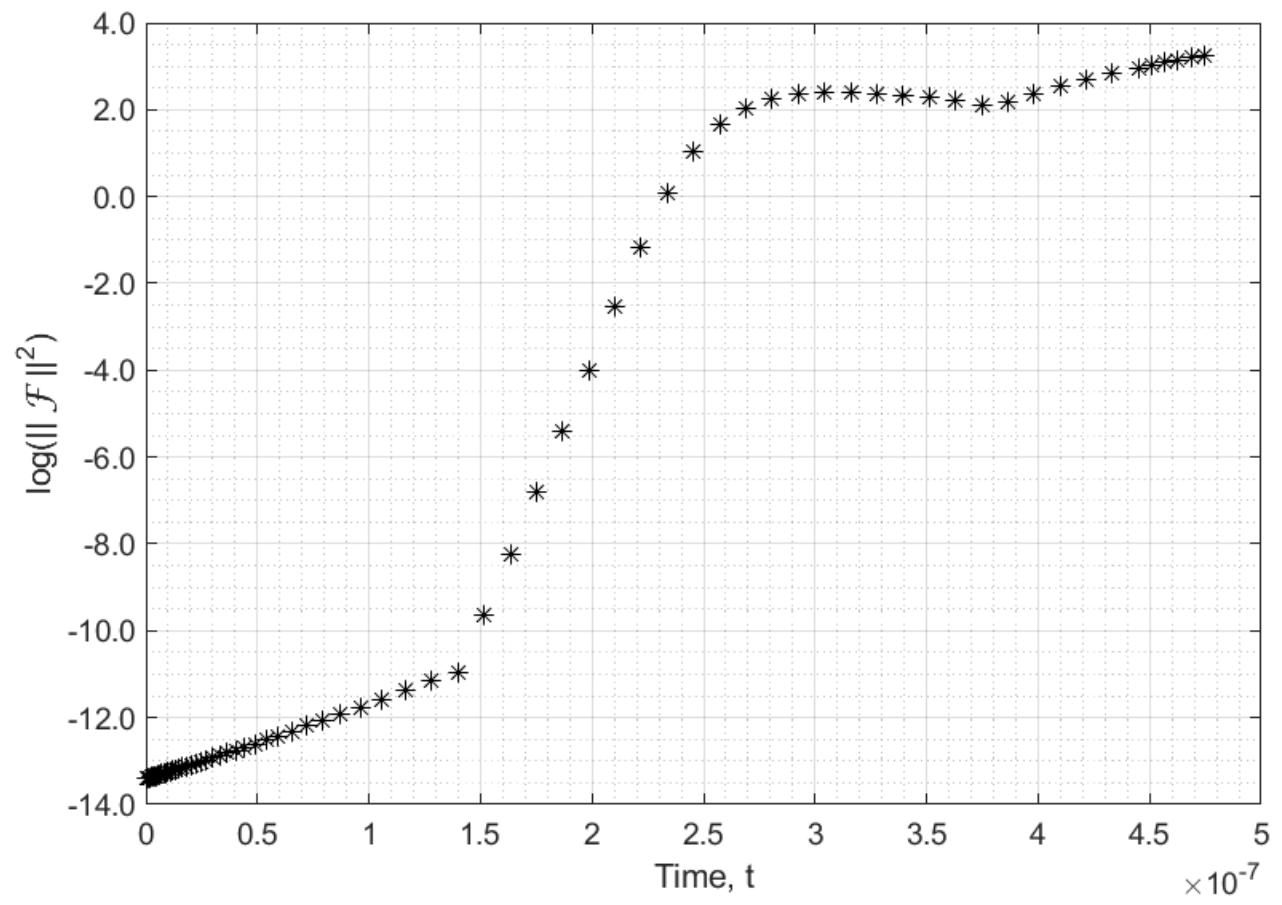

Figure 5.1-5 Maximum of amplitude spectrum of the same simulation as in Figure 5.1-1 (Case 5.1.1.B)

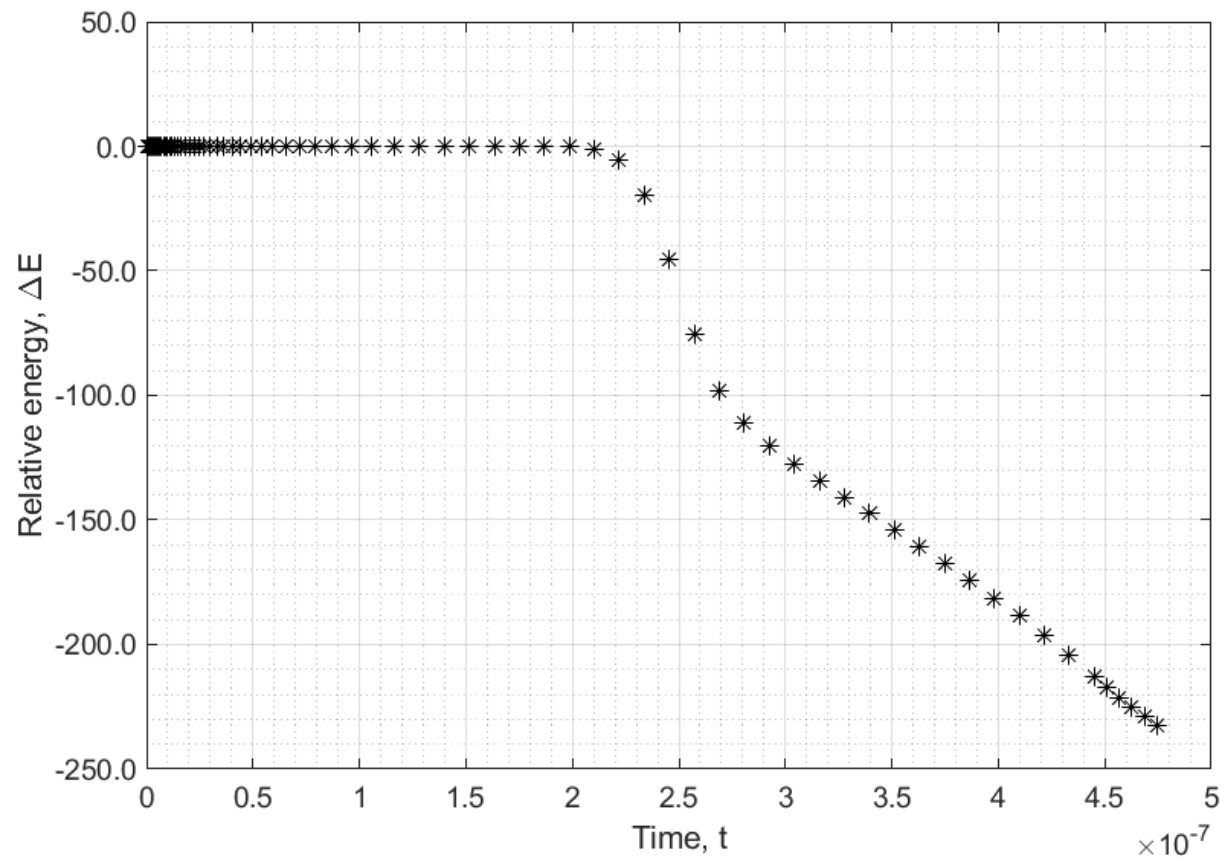

Figure 5.1-6 Relative energy the same simulation as in Figure 5.1-1 (Case 5.1.1.B) 

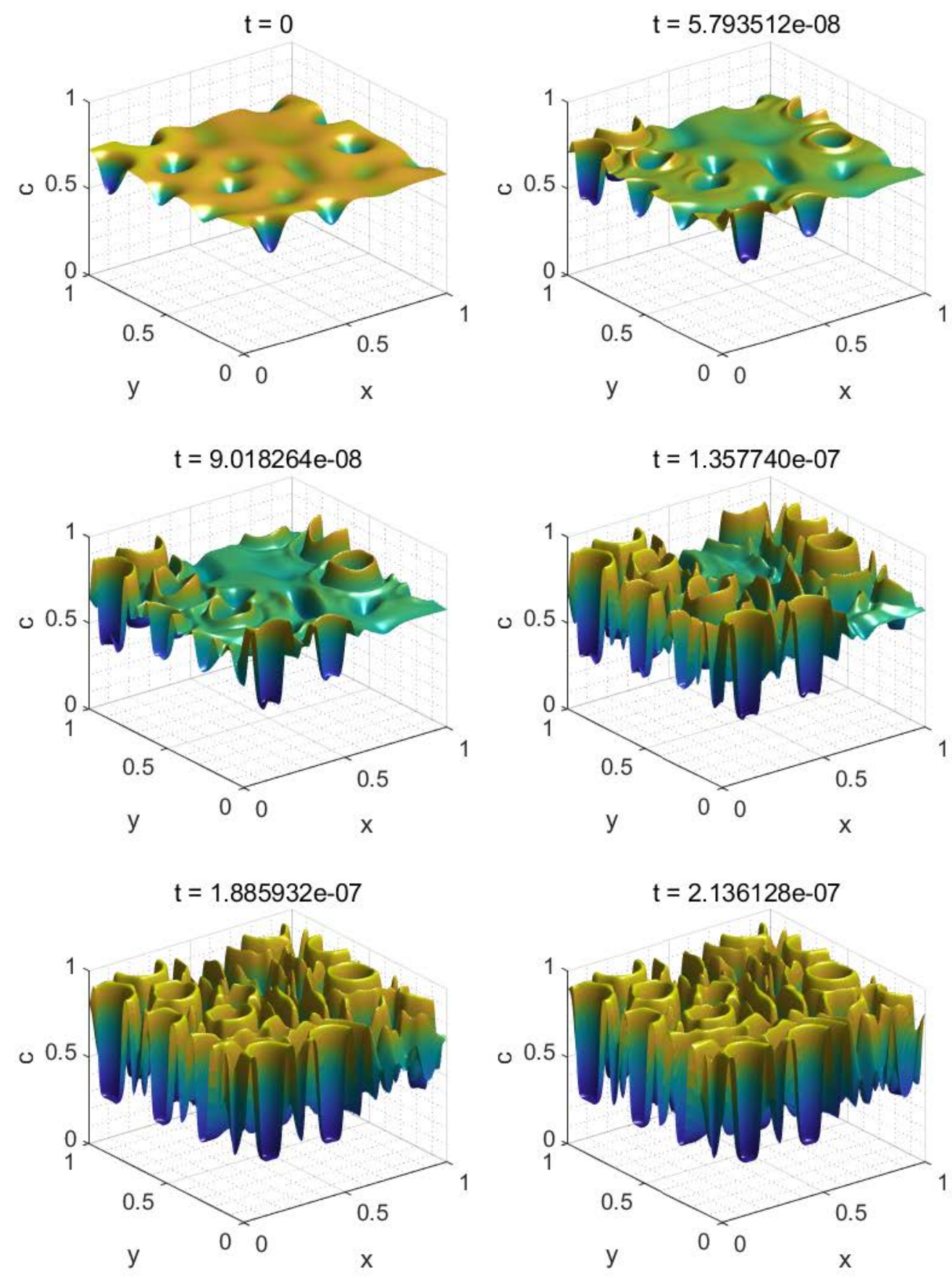

Figure 5.1-7 Concentration profile of second quench simulation after the first quench is carried out for $t=2.682418 \times 10^{-7}$ (Case 5.1.1.C) 

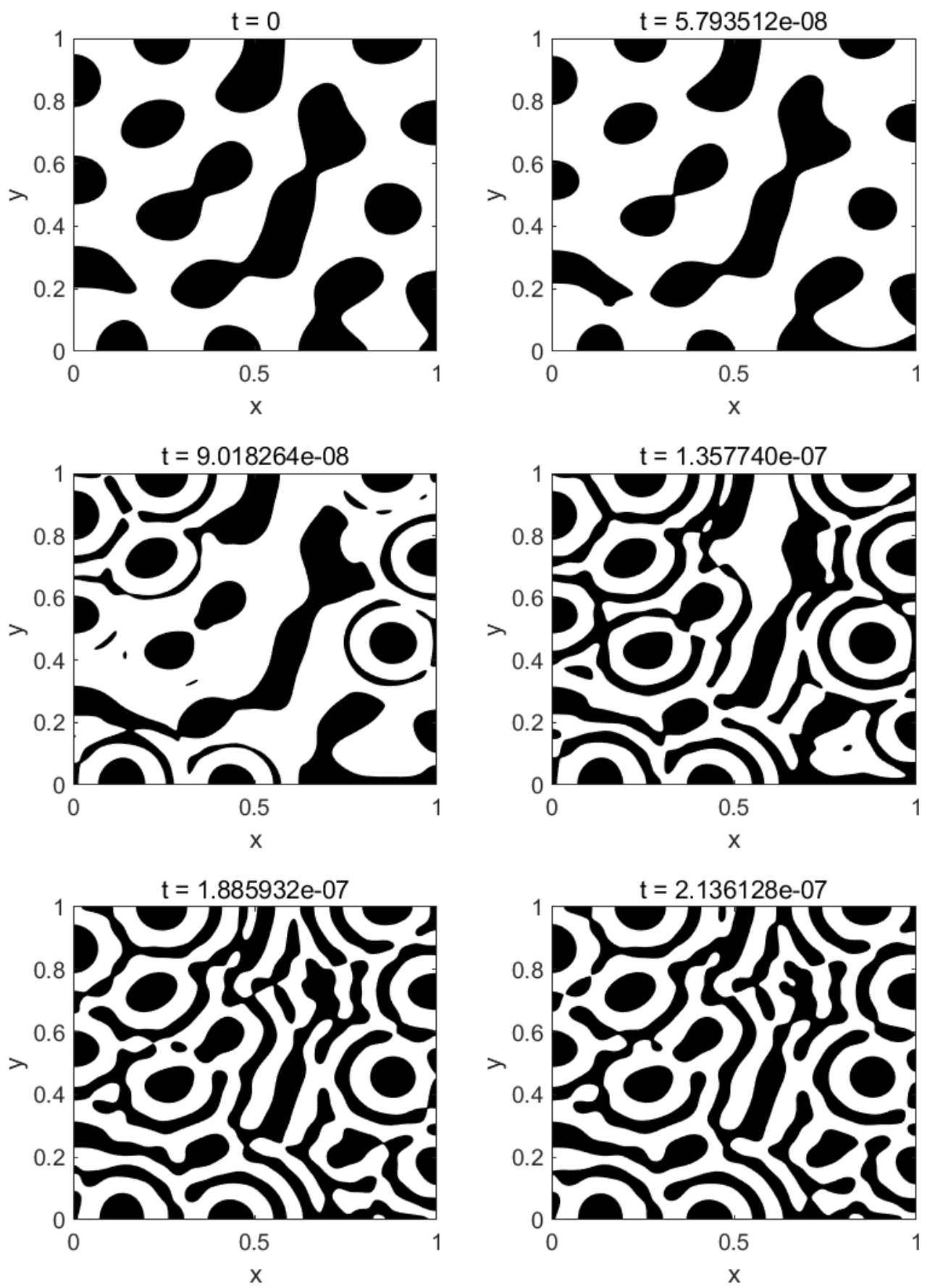

Figure 5.1-8 Contour plot of the same simulation as in Figure 5.1-7 (Case 5.1.1.C) 

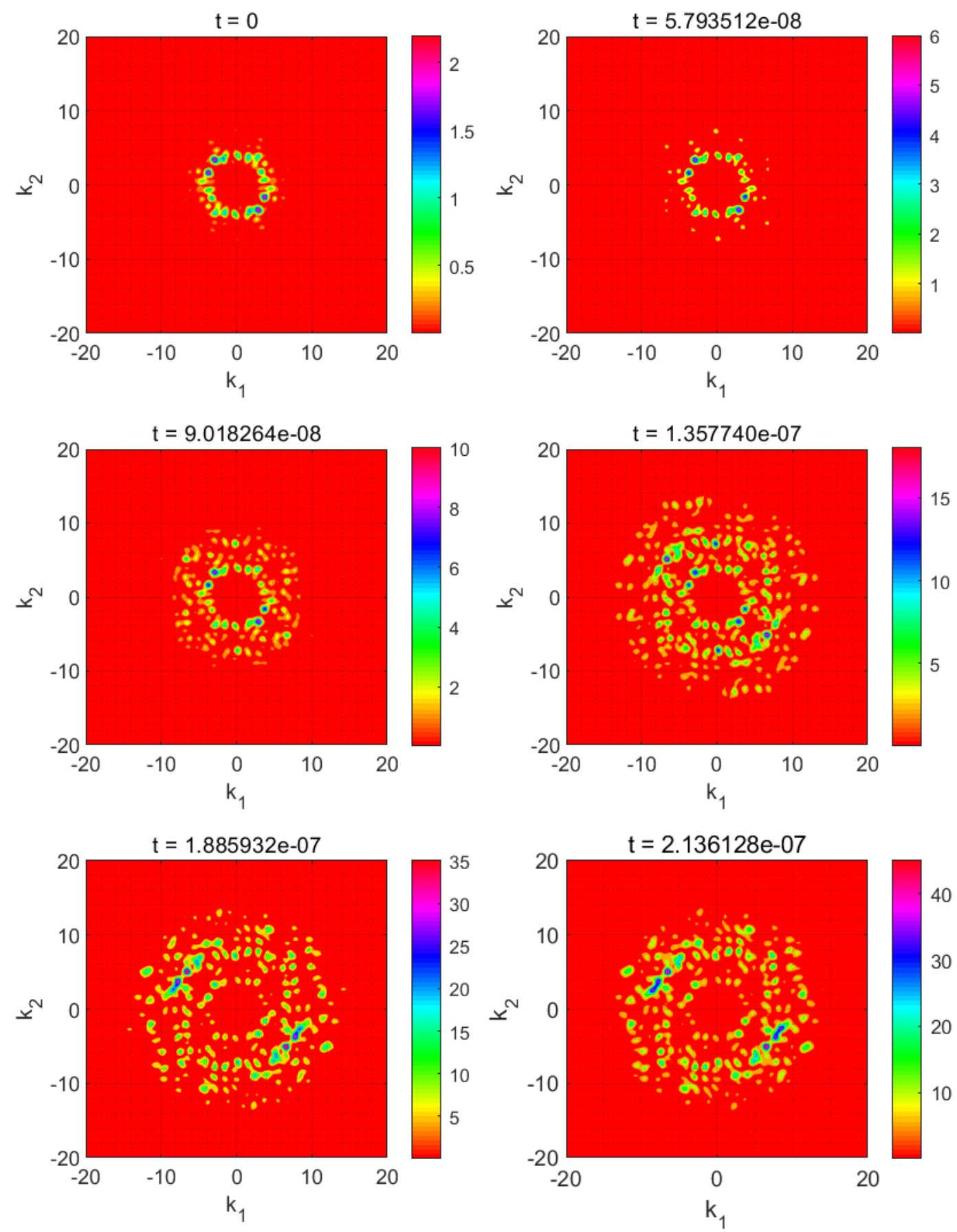

Figure 5.1-9 Amplitude spectrum of the same simulation as in Figure 5.1-7 (Case 5.1.1.C) 

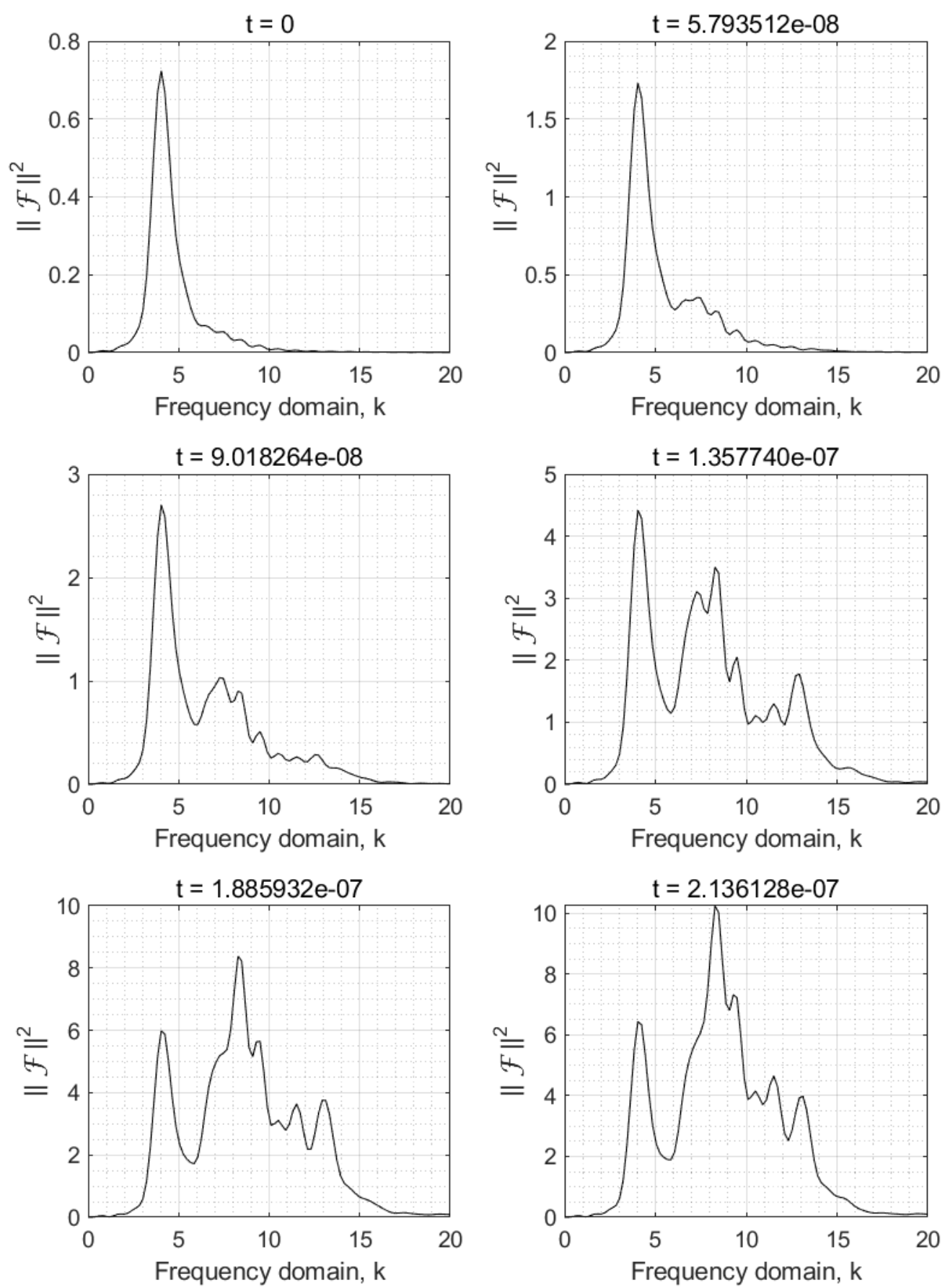

Figure 5.1-10 Circularly averaged amplitude spectrum of the same simulation as in Figure 5.1-7 (Case 5.1.1.C) 

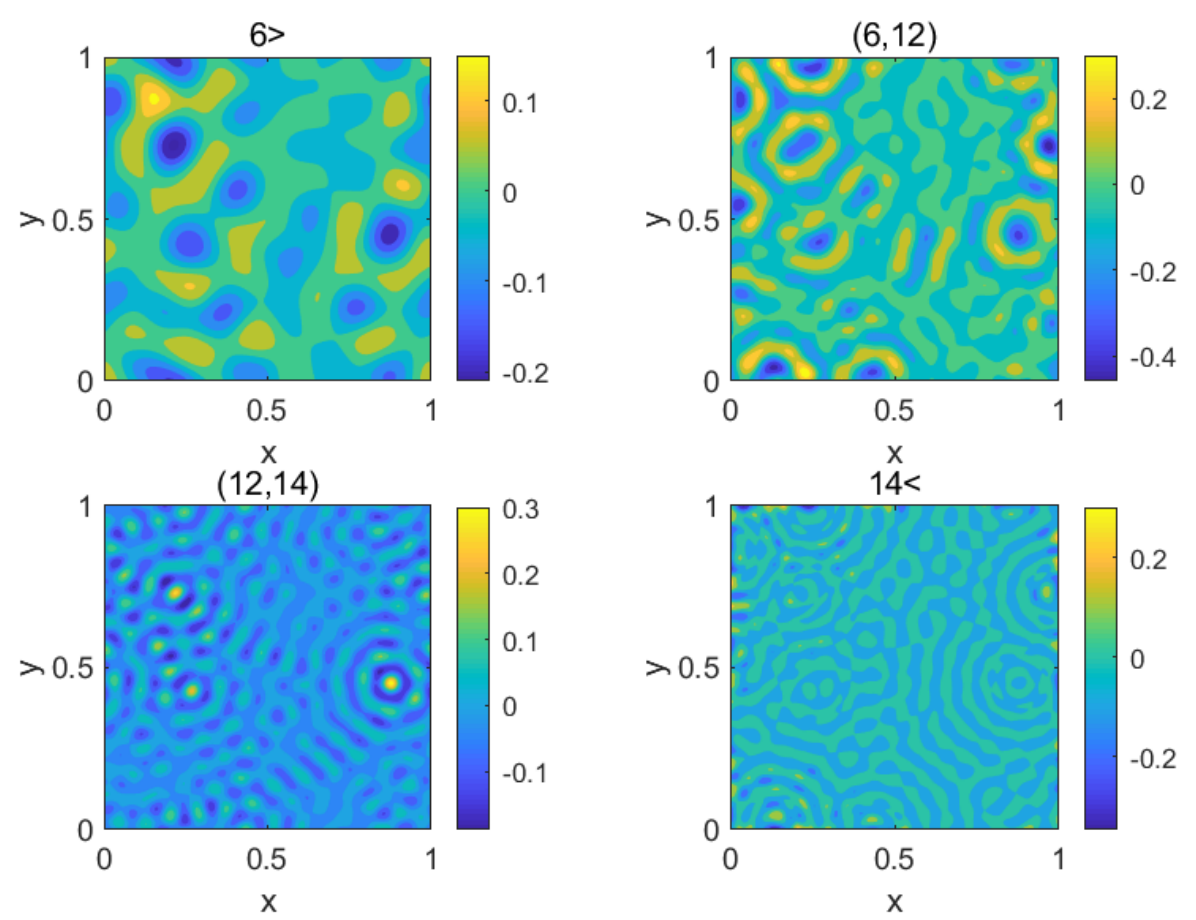

Figure 5.1-11 Concentration profile with band-pass filtered applied at $t=2.136128 \times 10^{-7}$ (Case 5.1.1.C)
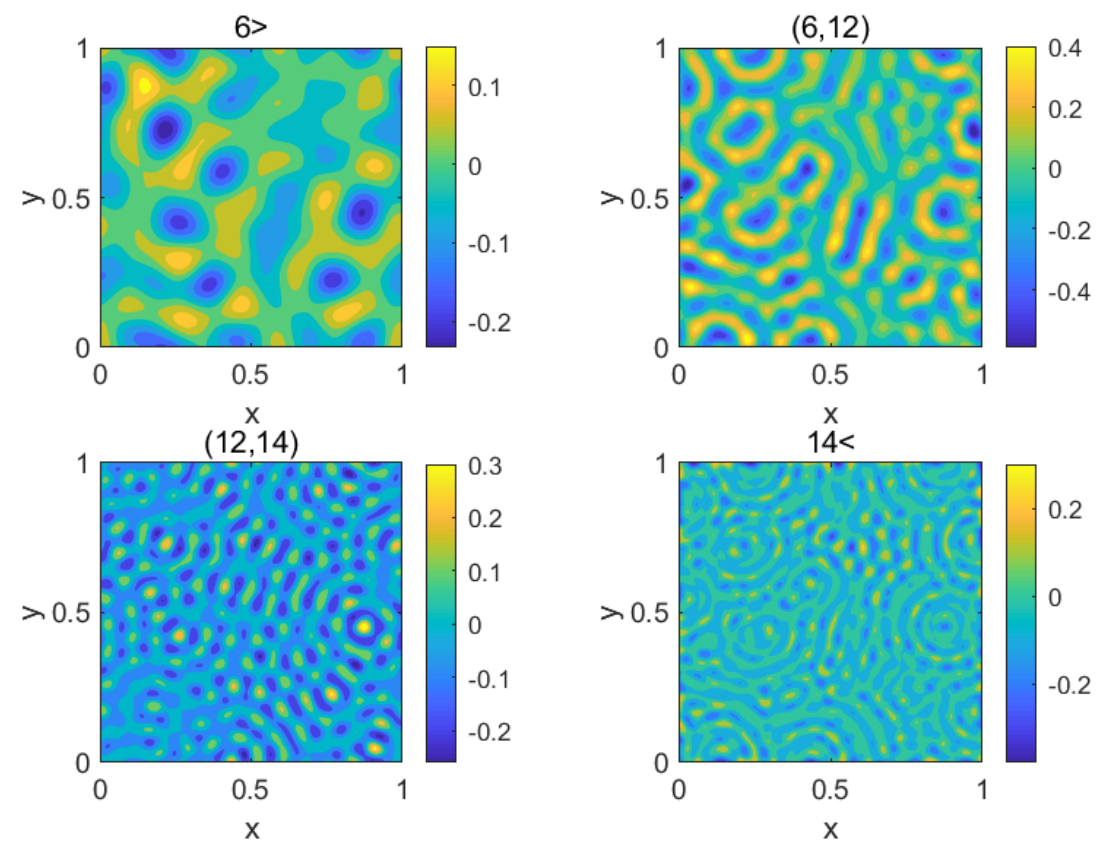

Figure 5.1-12 Concentration profile with band-pass filtered applied at $t=2.136128 \times 10^{-7}$ (Case 5.1.1.C) 


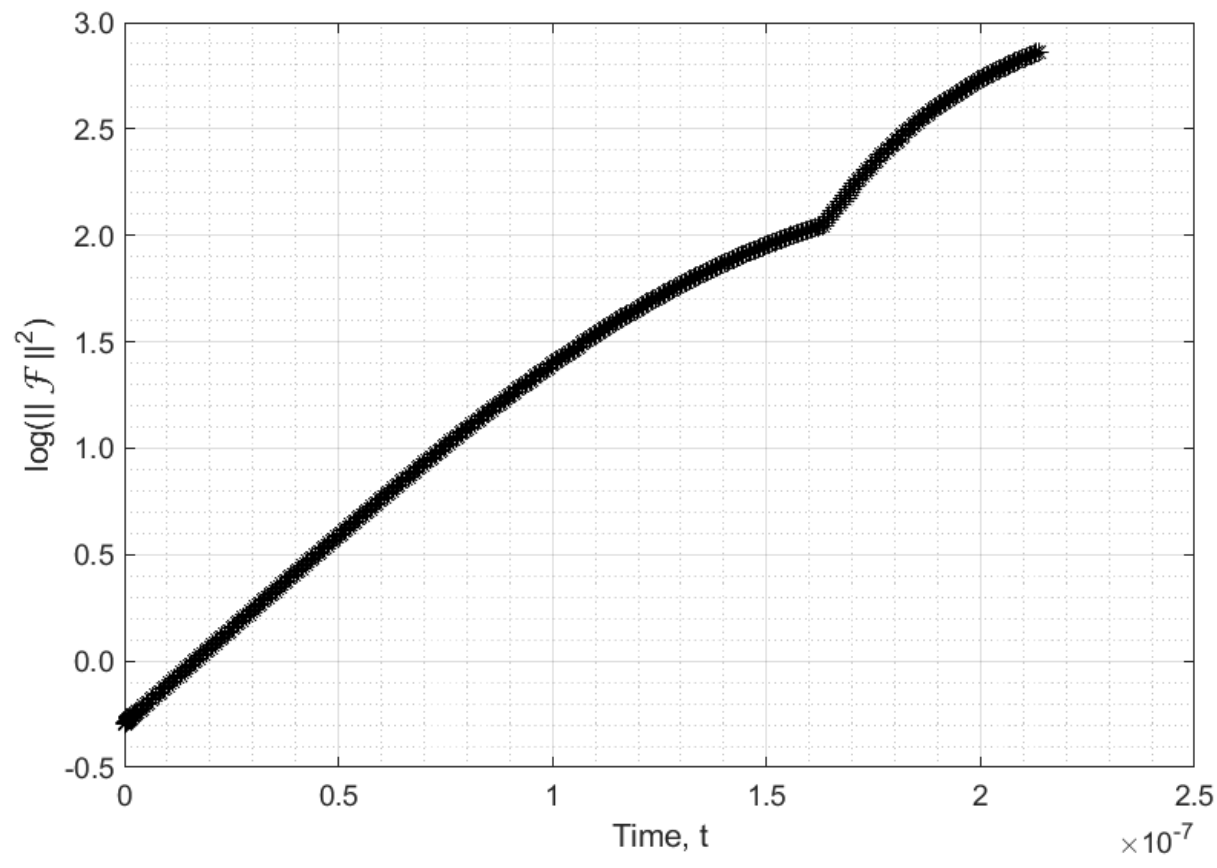

Figure 5.1-13 Log plot of the concentration maximum of the amplitude spectrum of simulation in Figure5.1-7 (Case 5.1.1.C)

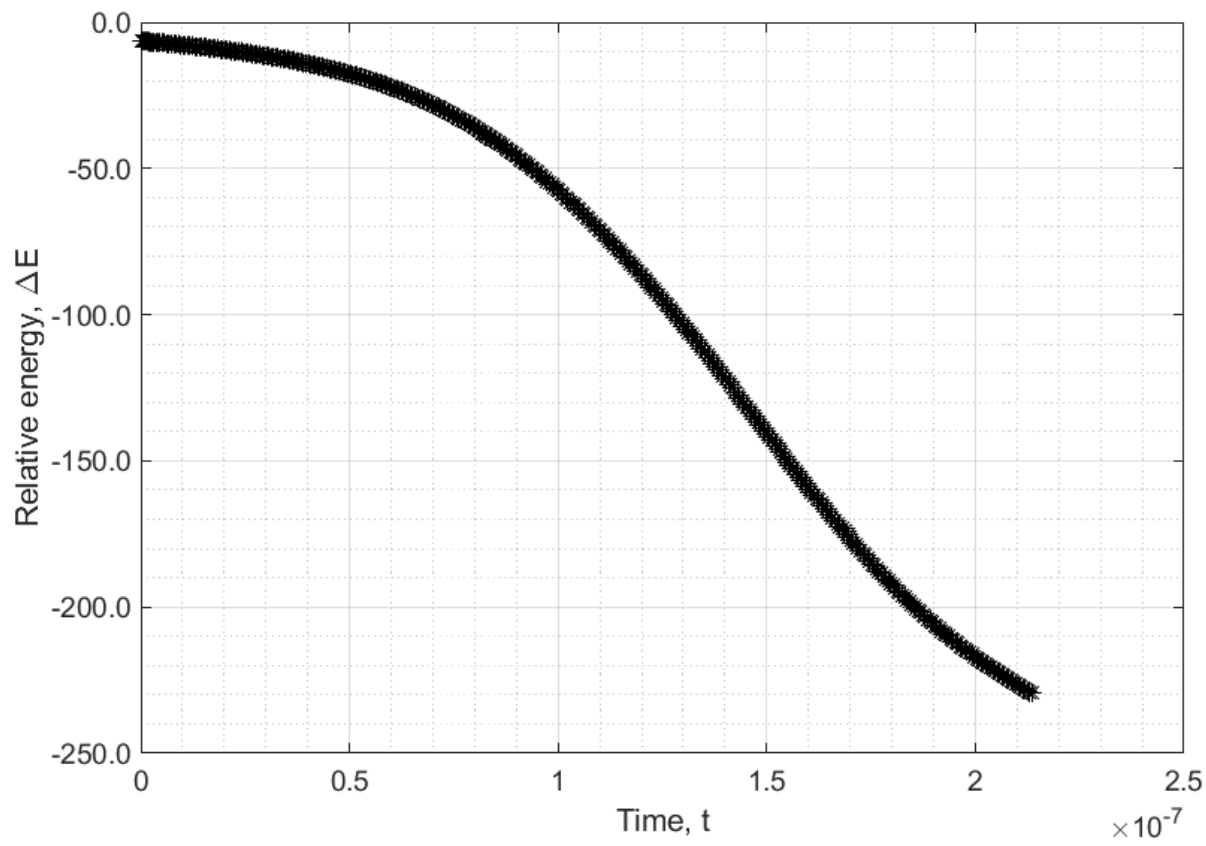

Figure 5.1-14 Relative energy of the simulation in figure 5.1-7 (Case 5.1.1.C) 

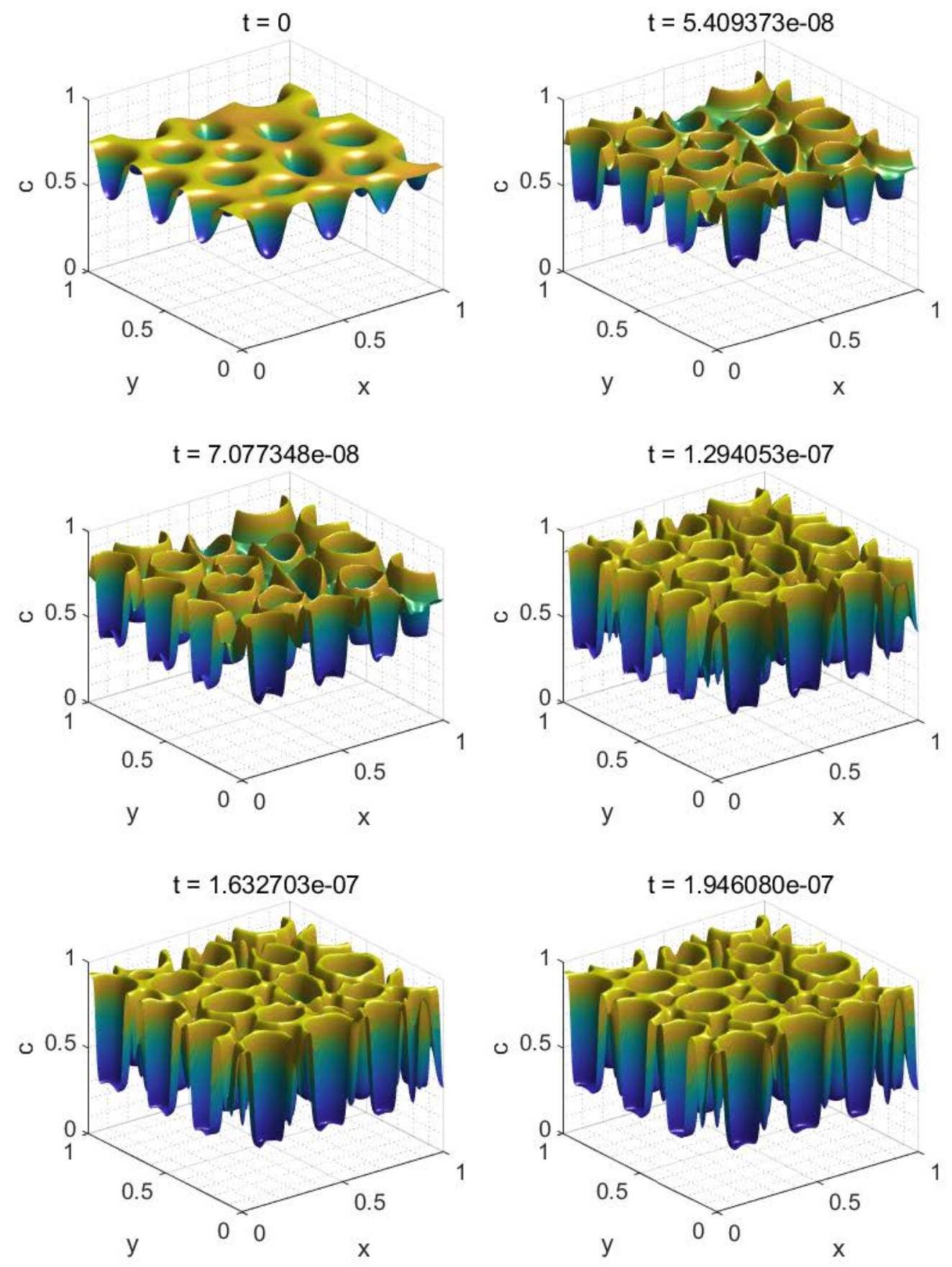

Figure 5.1-15 Concentration profile of second quench after the first quench is run until $t=$ 5.712641 × $10^{-8}$ (Case 5.1.1.D) 

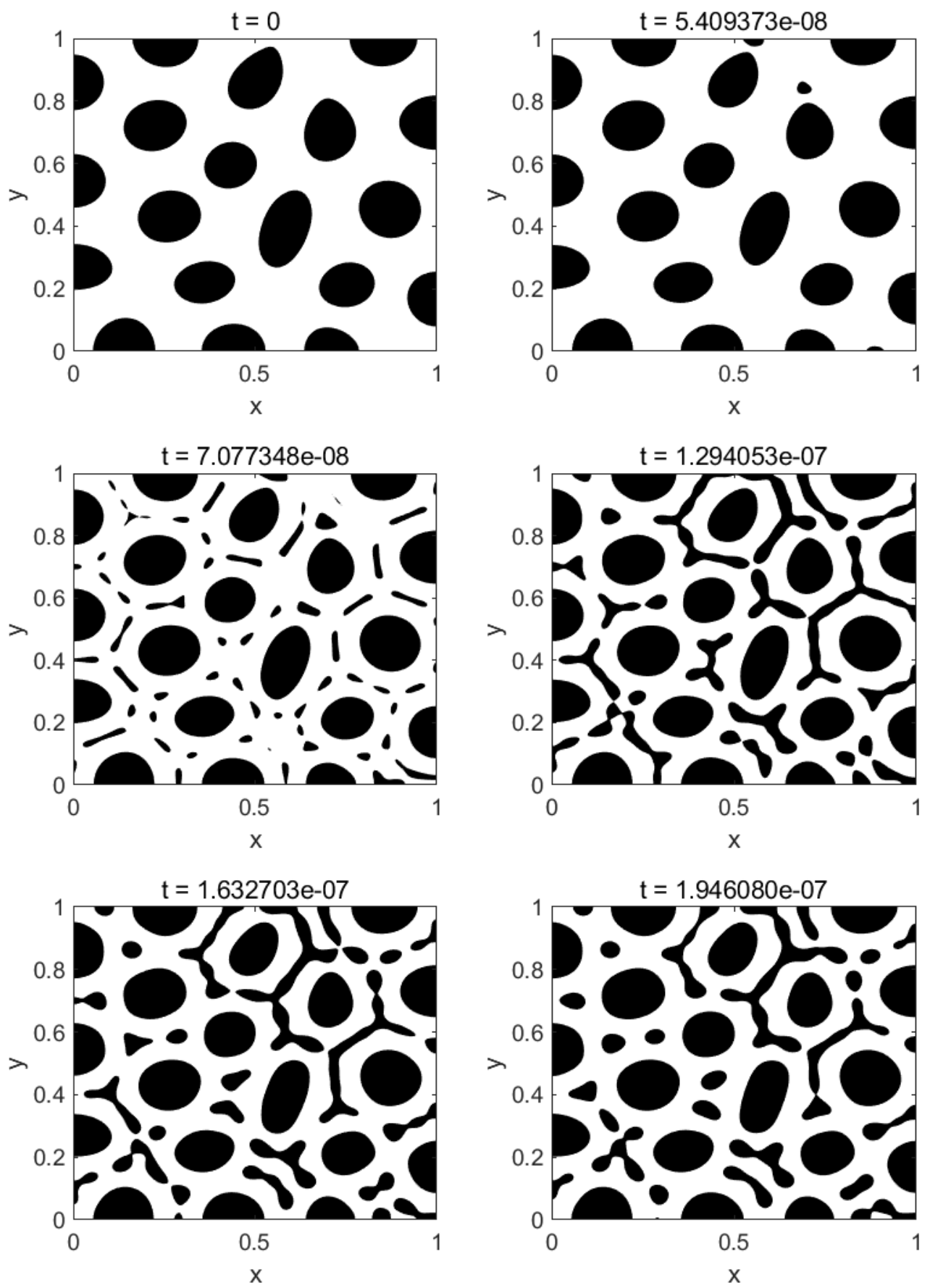

Figure 5.1-16 Contour plots of the same simulation as in Figure 5.1-15 (Case 5.1.1.D) 

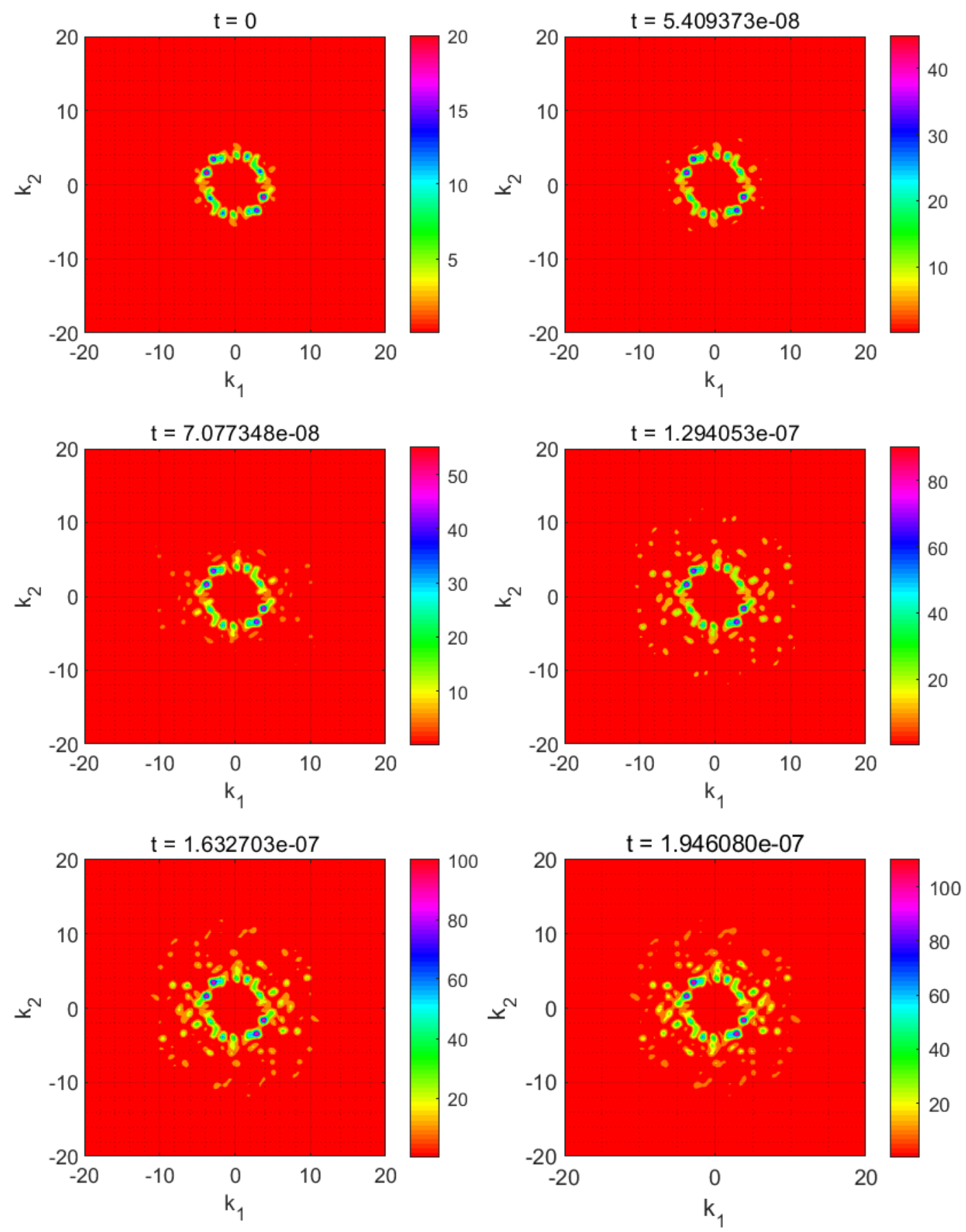

Figure 5.1-17 Amplitude spectrum of the same simulation as in Figure 5.1-15 (Case 5.1.1.D) 

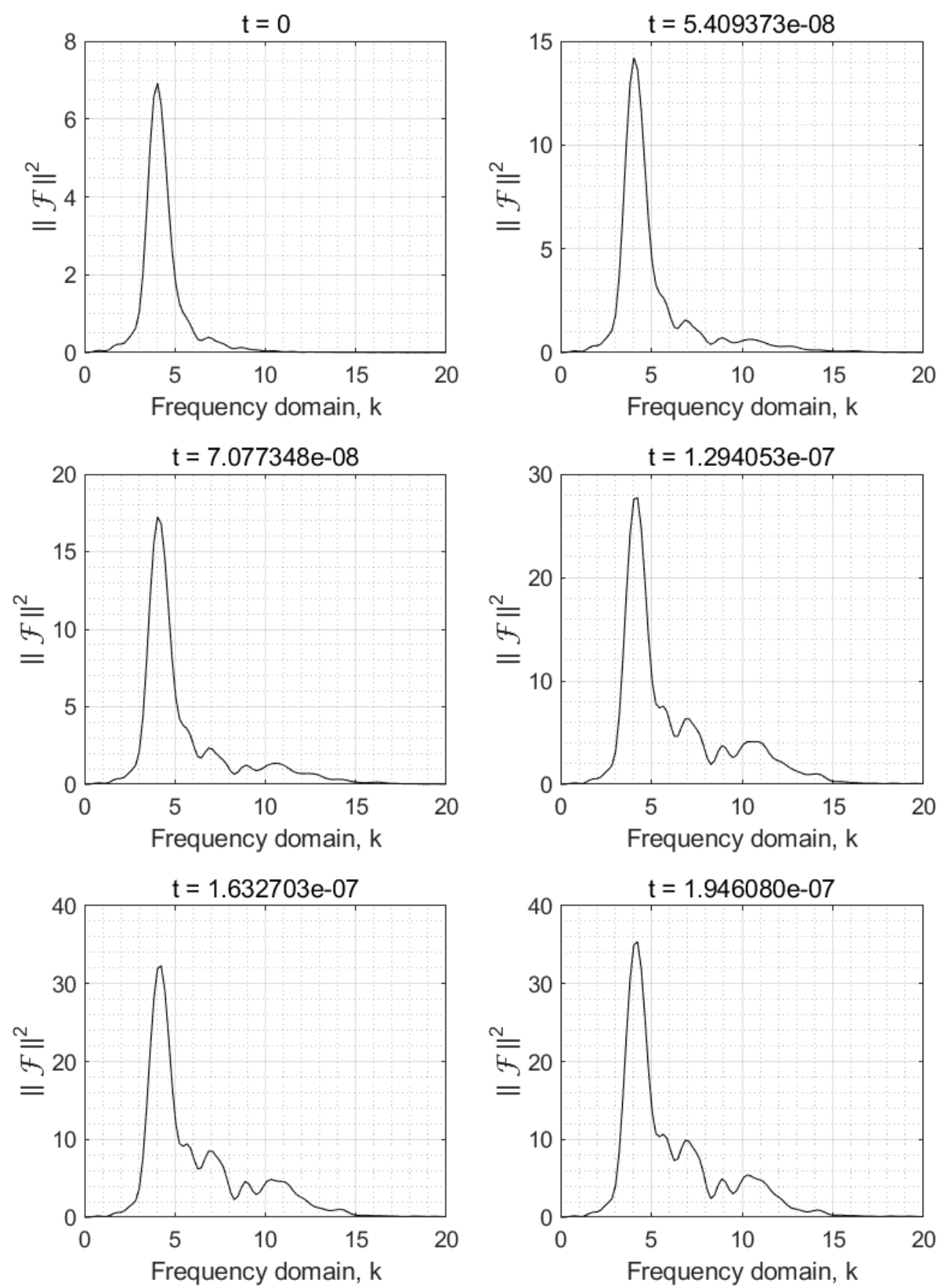

Figure 5.1-18 Circularly averaged amplitude spectrum of the same simulation as in Figure 5.1-15 (Case 5.1.1.D) 

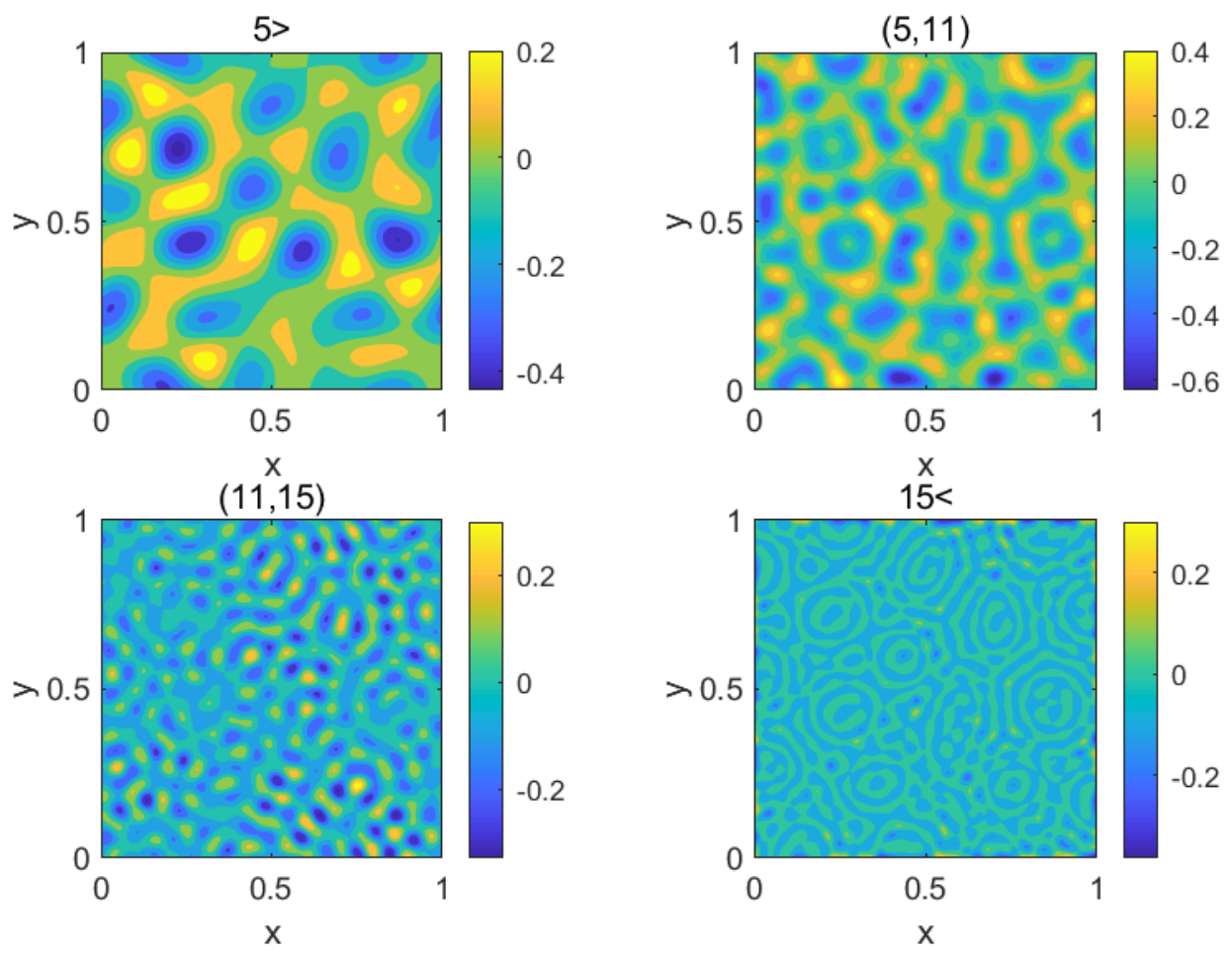

Figure 5.1-19 Concentration profile with band-pass filtered applied at $t=1.632703 \times 10^{-7}$ (Case 5.1.1.D)

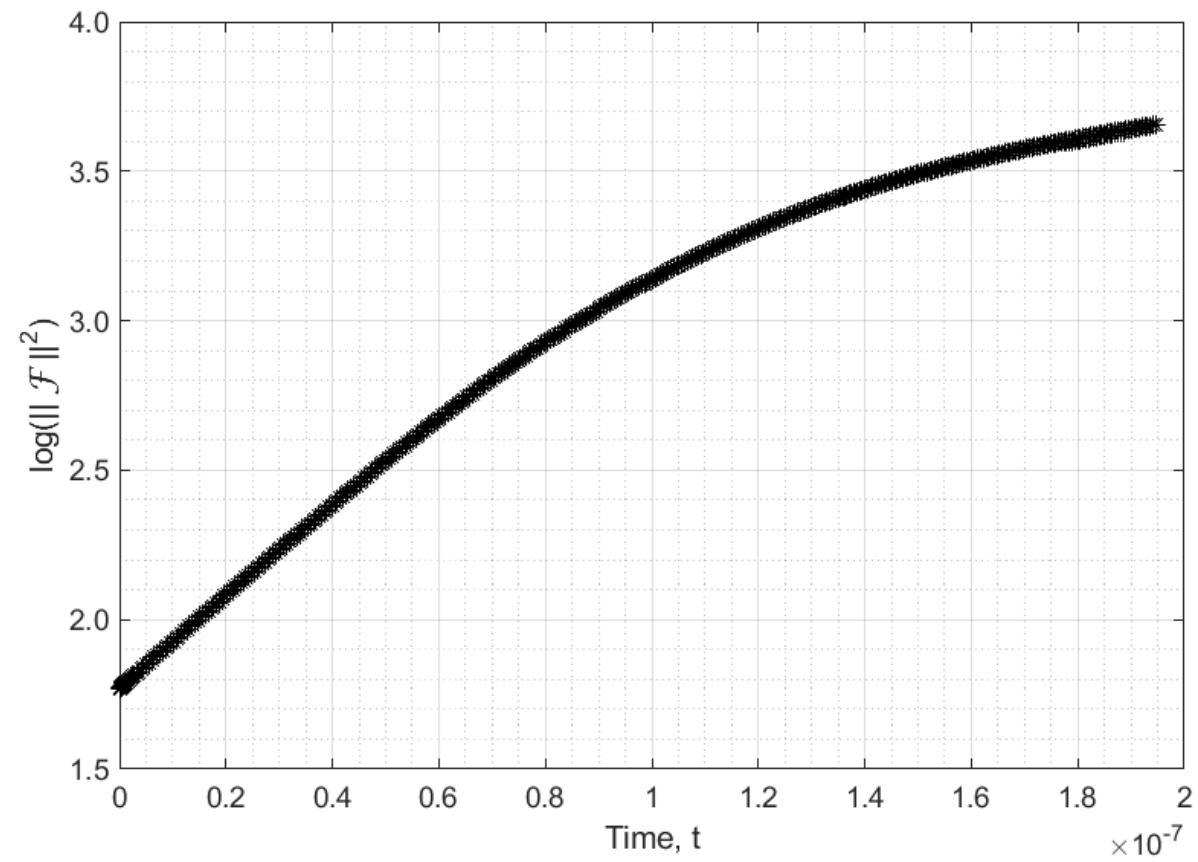

Figure 5.1-20 Log plot of maximum amplitude of the same simulation as in Figure 5.1-15 (Case 5.1.1.D) 


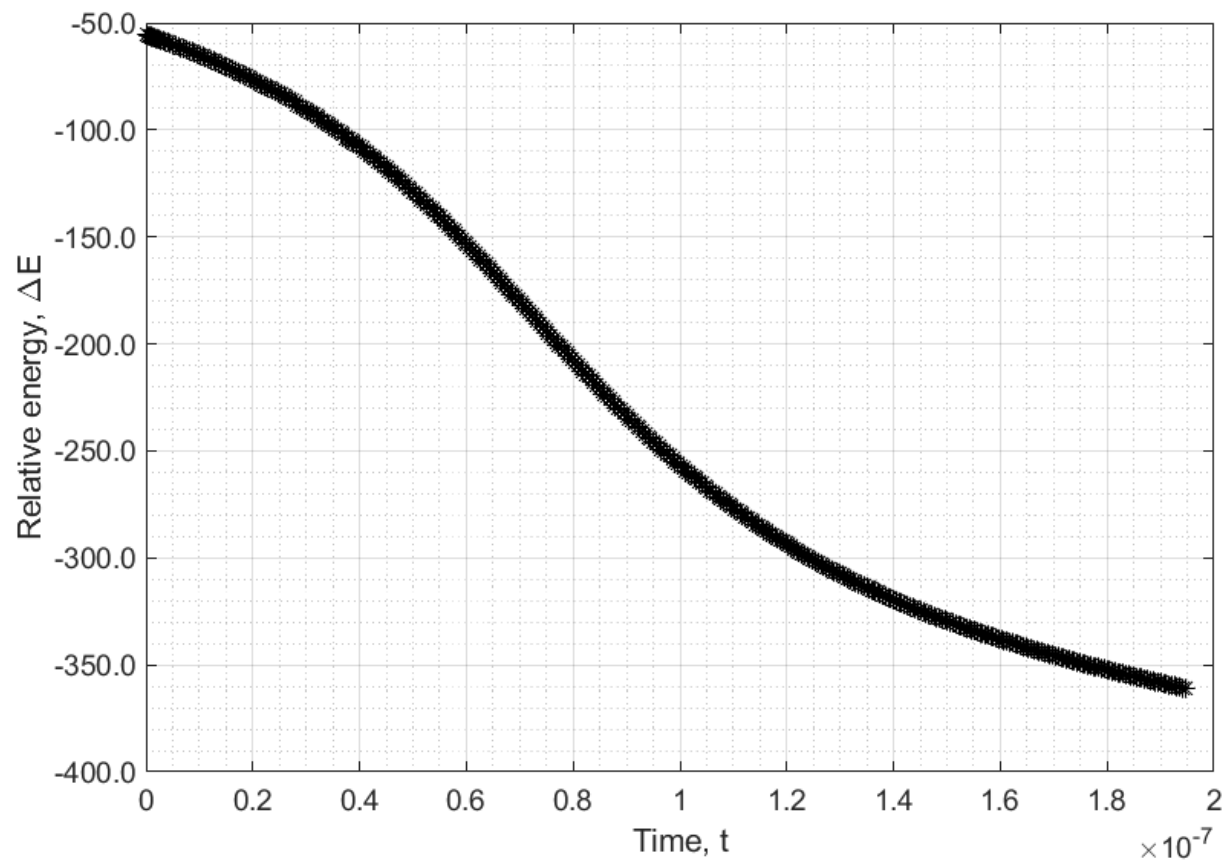

Figure 5.1-21 Relative energy of the same simulation as in Figure 5.1-15 (Case 5.1.1.D)

\subsubsection{Unstable first quench, metastable second quench}

So far, isotropic phase separation follows one principal rule that phase separation proceeds in such way that the overall energy, in the form pointed out by John W. Cahn, monotonically decrease. Furthermore, phase separation proceeds in such way that the configuration of the binary solution conforms to the shape that minimize the energy fastest. In relative stable late stage, the solution locally reaches close to two equilibrium concentrations as predicted by free energy. In this case, it is Flory-Huggins theory. This way, theoretically, the minimum energy a solution can achieve after an infinitely long time is when all of the following are satisfied

$$
\begin{gathered}
|\nabla c|^{2}=0 \forall \vec{r} \in \Omega \\
c=e_{1} \text { or } c=e_{2} 0 \forall \vec{r} \in \Omega
\end{gathered}
$$

Note that, the ratio of $c=e_{1}$ and $c=e_{2}$ can be well defined given the average concentration. 
This configuration can never be achieved in this thesis, nor in the experiment, because the solution will always contain some sort fluctuation, or gradient of concentration. Interfacial boundary between phases must always be present. Nonetheless, this still information can still give a good insight into how one can achieve a solution that is desired.

In this chapter, phase separation within metastable region of the solution with the simulation is discussed. The phase separation in metastable state must start with nucleation, an event that may occur independent of spinodal decomposition. Nucleation is achieved by some sort of noise or fluctuation. Once such nucleation is achieved, the growth proceeds spontaneously in such way that overall energy of the solution decreases monotonically. Because nucleation is a random event that takes into account statistical physics into model, and sometimes it can be an unlikely event or an event that can be hard to control, it is beyond the scope of this thesis. From engineering perspective, one may like to strategically carry out phase separation and generate the morphology as desired. At this point, it is appropriate to well define phase separation. Phase separation is said to be occurring when

$$
\frac{\partial}{\partial t} \int_{V}\left(c-c_{o}\right)^{2} \partial v>0
$$

That is, the variation of the overall concentration relative to average concentration is increasing over time.

Note that, in present model, only small fluctuation at the beginning is included as a noise. It has been assumed that this well mimics the uniform configuration of binary solution. In metastable state, for phase separation to occur, some sort of activation energy is required. In other words, the solution must have achieved a configuration that is different from a perfectly uniform solution. It is difficult with present model and specified parameters to initiate phase separation with single quench with only small noise at the beginning. However, because strategical engineering perspective is more of interest, phase separation is induced by double quench. That is, phase separation is first carried out within unstable region, the solution is brought to 
shallower quench that lies within metastable region. For this purpose, spinodal and binodal lines of phase diagram are deliberately calculated.

By the law of thermodynamics, metastable phase separation must always occur in a binary solution without any external influence that has achieved all of the following

$$
\begin{aligned}
& \int_{V}\left(c-c_{o}\right)^{2} \partial v \neq 0 \\
& E \leq\left. E\right|_{c=c_{\text {ave }} \forall \vec{r} \in \Omega}
\end{aligned}
$$

That is, the solution has achieved a non-uniform configuration whose overall energy is equal to or less than its counterpart with uniform configuration. If the solution is to proceed back to uniform configuration, it must first bypass a point at which $E>\left.E\right|_{c=c_{a v e} \forall \vec{r} \in \Omega}$, which is impossible without any external force.

The behavior of spinodal decomposition is predictable in a way that the solution always starts from certain uniform energy and then approach certain minimum energy as defined by diffusion coefficient $(D)$, polymerizations $\left(n_{1}\right.$ and $\left.n_{2}\right)$, average concentration $\left(c_{0}\right)$, entropy $(S)$, and uniform temperature $(T)$ with present model. Thus, one may first quench the solution to phase separate into certain configuration so that, upon second quench, the solution satisfies all of the conditions stated above. That is, one may strategically pick a solution whose minimum energy is far lower than energy of the uniform state in the second quench's environment. It is also reasonable to pick parameter so that the characteristic frequency is low in the first quench. This way, there is less interfacial energy in the beginning of the intermediate energy hence the solution approaches desired energy faster.

For this case, the following specific situation is considered. The solution is first quenched from uniform state to $D=10000, c_{\text {ave }}=0.2, T=0.26, n_{1}=n_{2}=1$ (Case 5.1.2.A) and is shown in Figure B - 2. For this specific condition, it has a uniform energy, $E_{o}=181.9074$, and minimum energy, $E_{\min }=-206.5608$. The temperature is then quickly raised to $T=0.26$, which lies within metastable region. Here, uniform energy, $E_{o}=-166.3341$, and minimum energy, $E_{\min }=-358.7308$. Thus, in the first quench, the solution is monitored and phase separation is 
stopped once it has achieved a configuration that yields $E \leq-166.3341$. Note that, because this condition is very close to spinodal line of phase diagram, the droplets phase separates at quite different rates since that lag-time is now longer. Hence, only one relatively apparent droplet is observed over a period of time, particularly at $t=3.264183 \times 10^{-5}$.

The configuration of iteration 123 , or at $t=3.264183 \times 10^{-5}$, in previous simulation for first quench (Case 5.1.2.A), where absolute energy has just gone below $E=-166.3341$, is taken then it is quenched to the same condition except , $T=0.29$ (Case 5.1.2.B). The process is shown in Figure 5.1-22. By observing the peak at $x=0, y=0.15$, it continues to grow until close to the corresponding equilibrium value (Approximately $t=6.495712 \times 10^{-7}$. Once it has reached close to the equilibrium value, it starts to widen in area until the end of the simulation at $t=2.383637 \times 10^{-6}$. Immediately next to it is the circular area whose concentration is lower than the average value. Contour plot shown in Figure 5.1-23 reveals that the configuration from the starts, $t=0$, does not change significantly over time. The positioning of each droplet from the first time frame can be easily identified throughout the entire simulation. Some droplets, e.g. at about $x=0.8, y=0.85$, starts to shrink later on. But such rate is slow compared to the growth of the peak at $x=0, y=0.15$. Figure 5.1-25 shows the circularly averaged structure factor of the simulation. First, it has one apparent, high peak corresponding to characteristic frequency from simulation simulation, $f_{c}=3.8183372$. This peak seems to decrease initially until $t=4.288856 \times 10^{-7}$, the it starts to increase back up. More remarkably, broad peak immediately on both sides of this initially sharp peak starts to grow, monotonically increasing on the left hand side, while monotonically decreasing on the right hand side. Recall that, because growth factor is calculated based on relative concentration rather absolute concentration (otherwise, one would observe very high peak at $f=0$ ), growth factor at $f=0$ is always equal to zero with present formula, as discussed previously. Also, by comparing to the shape of the growth of structure factor in nucleation and growth as shown by Liu and Kiran [13], it is reasonable to conclude that the phase separation behavior is the characteristic of nucleation and growth. Figure 5.1-24 shows more in-depth insight into the structure factor. Instead of sharp well-defined circular ring, a concentric pattern with monotonically decreasing values as one moves further away from $k_{1}=k_{2}=0$, with the exception of the center region 
for the same reason explained. This further asserts the observation of nucleation and growth. As revealed in Figure 5.1-26, the maximum value of structure factor initially decrease then increase. Such increase in the value is as if the initial characteristic frequency is waiting for broad range of monotonically decreasing frequency to catch up and then starts to grow. Maximum of structure factor is lowest at approximately $t=4.288856 \times 10^{-7}$. This is the duration in which the solution attempts to conform to the new favorable configuration for growth, which is shown as monotonically decreasing structure factor over frequency domain. Once it has conformed to its shape, the growth becomes favorable and the peak approaches closer to the equilibrium value. This theory is further supplemented by the energy value as shown in Figure 5.1-27. As expected, the energy monotonically decreases at all time. As the simulation is deliberately started in already phase-separated state with relative energy being equal to zero, the binary solution will never go back to uniform configuration. From around $t=$ $4.288856 \times 10^{-7}$, decrease in energy becomes steeper. This is the duration in which the peak attempts to reach closer to equilibrium values. After all that, it is followed by relatively decrease in energy, which corresponds to widening of the droplet. Given that minimum energy exists, this seemingly linear line of decrease in energy must be slowed at some point. In both plots of maximum amplitude of structure factor and relative energy, the lag-time in which the solution conforms to new morphology before quick phase separation is seen (i.e. the decrease in the amplitude of structure factor and relatively slow drop in relative energy at the beginning). It is followed by faster phase separation. 

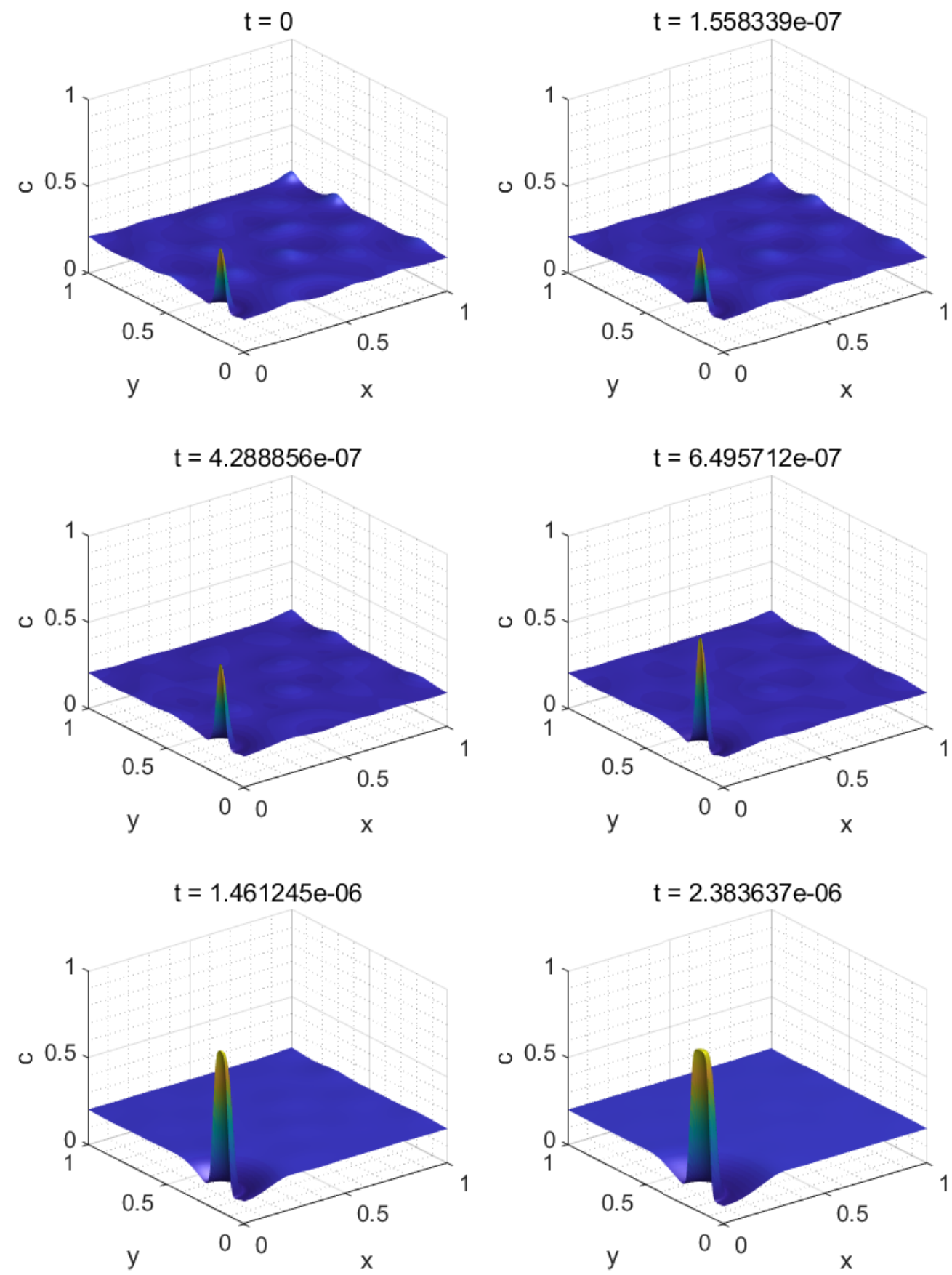

Figure 5.1-22 Concentration profile of metastable second quench for $D=10000, c_{\text {ave }}=$ $0.2, T=0.29, n_{1}=n_{2}=1$ where $c_{o}$ is obtained from Case 5.1.2.A at $t=3.264183 \times 10^{-5}$ (Case 5.1.2.B) 

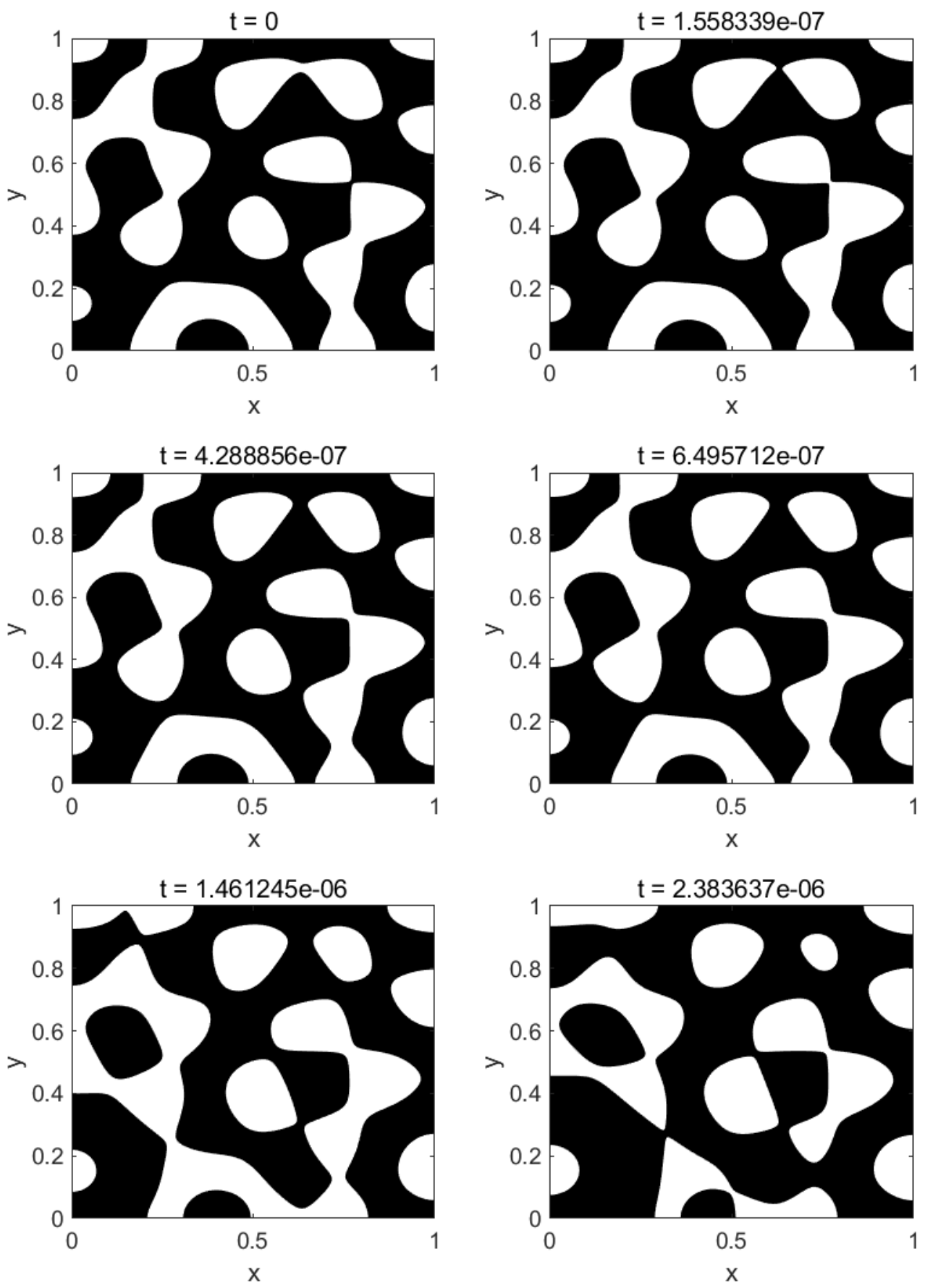

Figure 5.1-23 Contour plots of the same simulation as in Figure 5.2-22 (Case 5.1.2.B) 

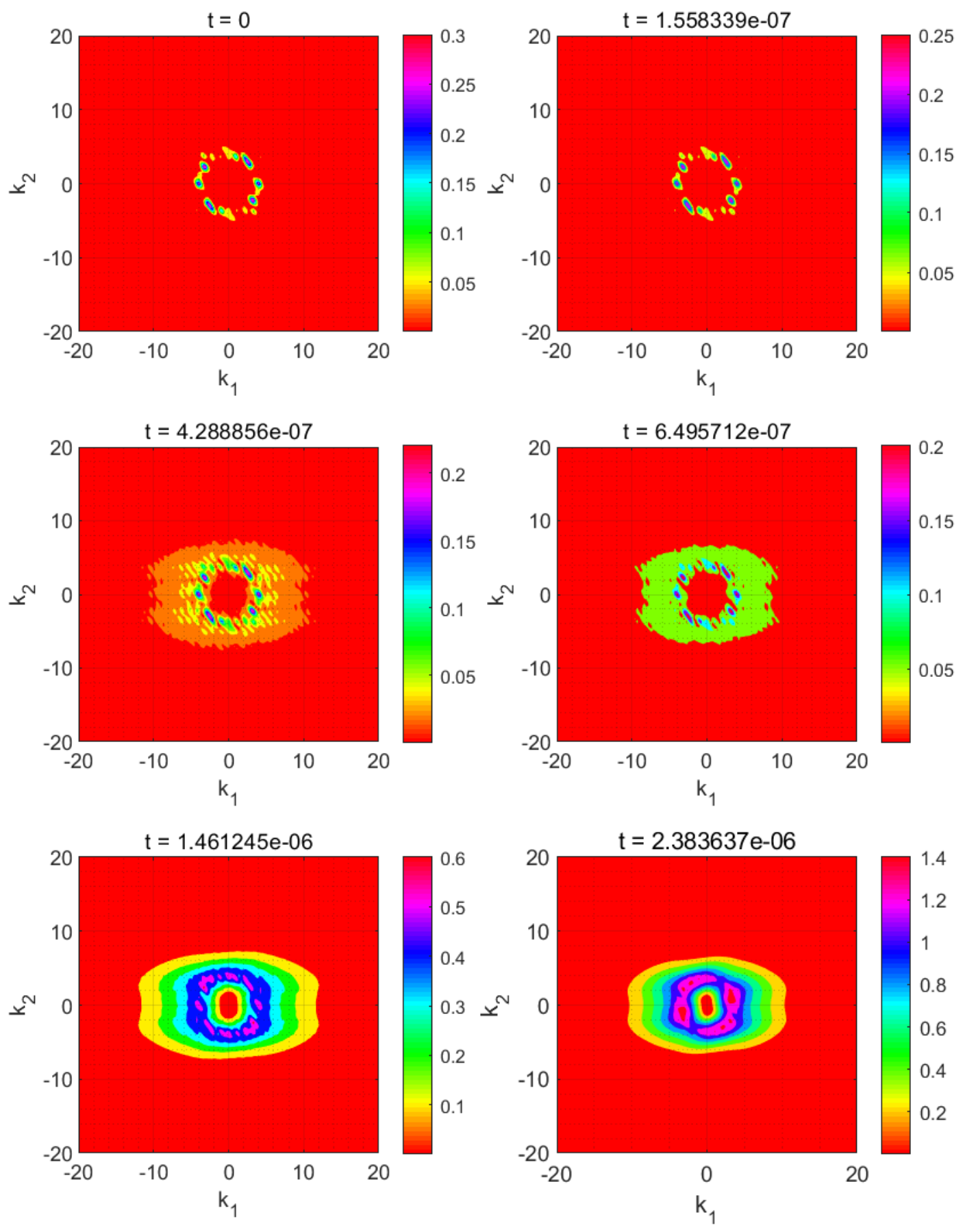

Figure 5.1-24 Amplitude spectrum of the same simulation as in Figure 5.2-22 (Case 5.1.2.B) 

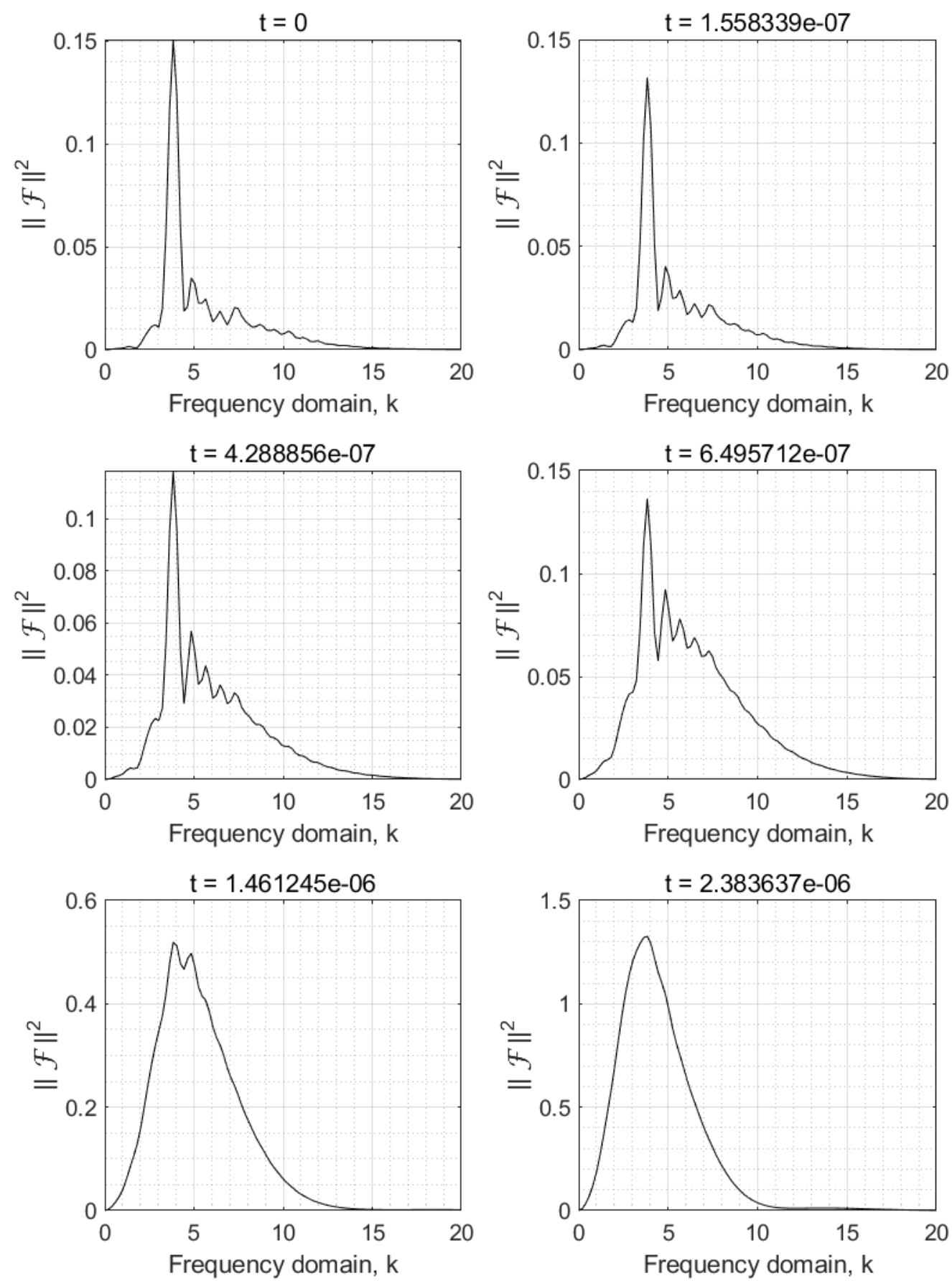

Figure 5.1-25 Circularly averaged Amplitude spectrum of the same simulation as in Figure 5.2-22 (Case 5.1.2.B) 


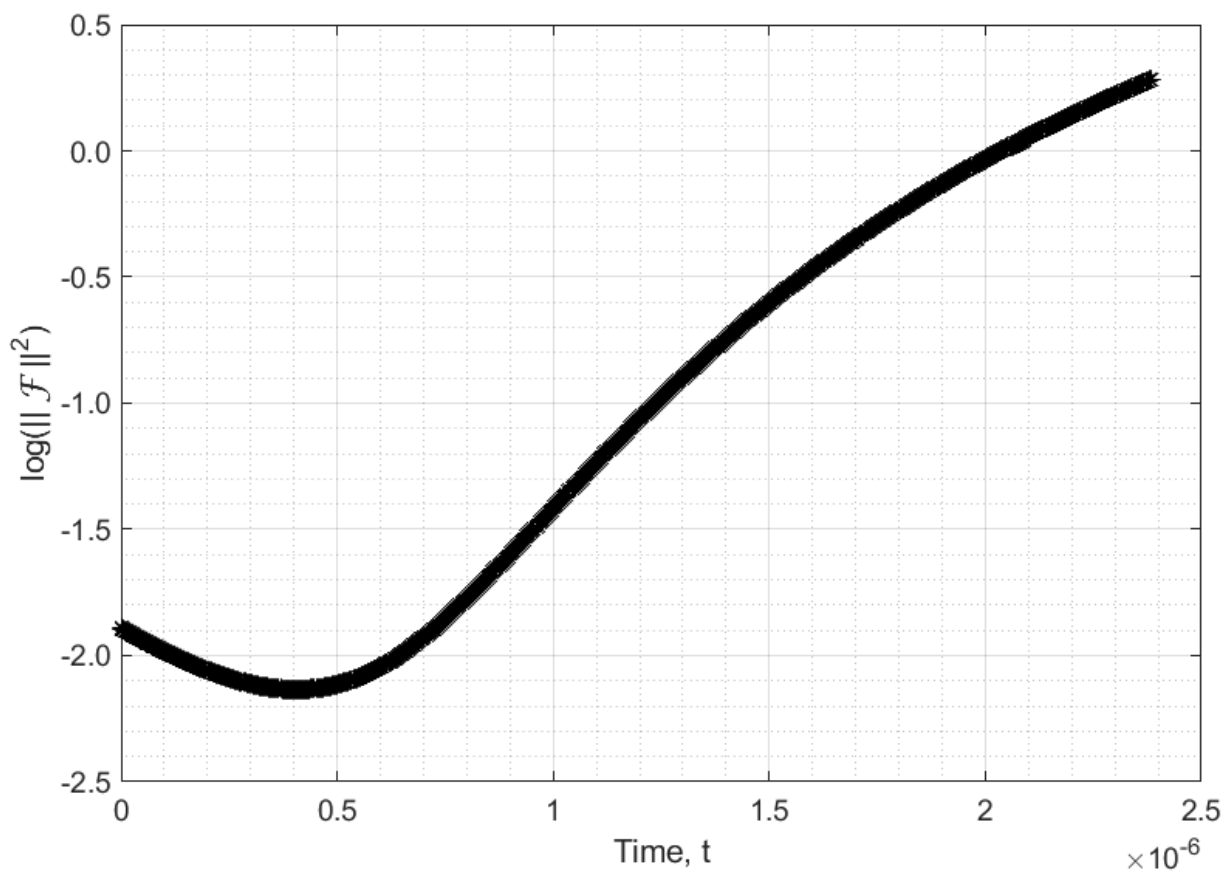

Figure 5.1-26 Log plot of maximum amplitude of the same simulation as in Figure 5.2-22 (Case 5.1.2.B)

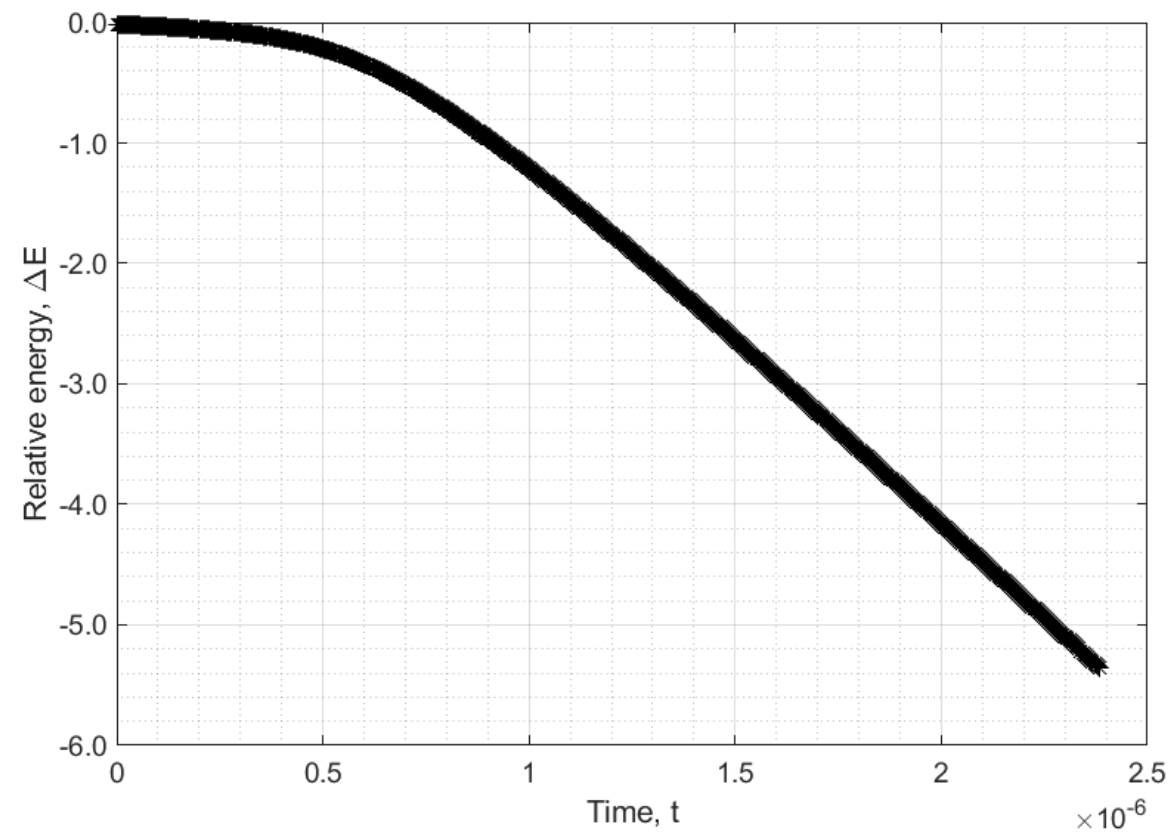

Figure 5.1-27 Relative energy of the same simulation as in Figure 5.2-22 (Case 5.1.2.B) 
While phase separated state with zero or negative relative energy will thermodynamically guarantee the growth with droplets acting as nucleation, if the solution starts from some positive relative energy in metastable region, it may or may not cross the relative energy of zero to become uniform or phase separated, respectively.

In order to demonstrate this, the configuration obtained from simulation for $D=$ 10000, $c_{\text {ave }}=0.3, T=0.3, n_{1}=n_{2}=1$ (Case 5.1.2.C), which is shown in Figure $\mathrm{B}-3$, with time frame $t=5.318622 \times 10^{-6}$ is taken. This is the beginning of the intermediate stage. The temperature is then quickly raised to $T=0.35$ (Case 5.1.2.D). In this condition, this configuration has relative energy of $E_{r}=32.0601$. By observing Figure 5.1-34, one may observe relatively quick drop in the energy in the initial step until around $t=8.0 \times 10^{-7}$. Around this time, relative energy has come close to zero and slowed down. The overall process can be visualized in Figure 5.1-28. Here, most of the droplets shrink quickly initially. However, at $t=1.50855 \times 10^{-6}$, droplets have almost disappeared or coarsened but some droplets persisted longer than the other. This trend keeps going until $t=2.247859 \times 10^{-6}$. This is when relative energy has become negative for the first time. After this time, the droplets that persisted for long time grows quickly, while the others continue to disappear. At $t=$ $5.232756 \times 10^{-6}$, the droplets at the boundary has grown faster than two growing droplets within the internal layer. This is because the droplets at the boundary has interfacial energy advantage. Nonetheless, the growth of two internal droplets eventually catch up as shown at $t=1.368102 \times 10^{-5}$. Two color contour plot in Figure 5.1-29 shows that every droplet simply coarsens over time. The droplets that are shrinking gradually conforms to co-continuous shape, well growing droplets retain droplet shape. Figure 5.1-30 shows the corresponding characteristic frequency for this particular double quench simulation. A sharp ring with radius 5.866149663 is seen at the beginning, $t=0$. This is the characteristic of single quench at $D=$ $10000, c_{\text {ave }}=0.3, T=0.3, n_{1}=n_{2}=1$ as predicted by linear assumption. Over time, this ring keeps shrinking in its altitude until just before relative energy becomes negative. At $t=$ $1.50855 \times 10^{-6}$, the ring at the start has shrunk in altitude, but the ring has formed circular 
region with approximately similar altitude inside it. Thus, the phenomenon in which structure factor with lower frequency has been evolving over time can be witnessed. Only after relative energy has reached negative value, $t=2.247859 \times 10^{-6}$, it seems that amplitude of lower frequency is becoming more dominant than surrounding area. The further away from the origin, the lower the structure factor is, as the simulation progresses. Thus, there is phase separation in such way that the structure factor decreases monotonically with the frequency. Please note that, Fourier transform formula here considers relative concentration rather than absolute one, thus the amplitude at the center is zero instead of a high value as expressed as dirac delta function. Figure 5.1-33 shows the log plot of the maximum of structure factor. There is a sharp kink at about $t=1.5 \times 10^{-6}$. This is the moment when the amplitude at about the characteristic frequency shrinks lower than the amplitude at the lower frequency, which is rising. It remarks the moment when new morphology has become important than the original one. The broad peak at low frequency will keep rising then approach more stationary state. Figure 5.1-31 is also appropriate for this isotropic double quench. At iteration $t=1.50855 \times$ $10^{-6}$, the significant peak has broadens, especially on the lower frequency side. There are three dominant peaks here at about $f=2.1,4$, and 6 . While the peak at $f=2.1$ is typical of metastable growth i.e. the structure factor at the lower frequencies, and the peak at $f=6$ is simply the remain of the initial condition, the peak at $f=4$ seems to be the result of two factors: First, at $t=0$, there is a slight amplitude is already present at this frequency. Second, it seems to rise as the result of droplets disappearing and enlarging, a phenomenon similar to unstable single quench in late stage. Recall that, in typical late stage, the most dominant structure factor does not shift to lower frequency gradually, but it is a step-by-step process. That is, as the droplets merge, new droplets with lower frequency result. Since the frequency is lower than characteristic frequency, it is expected to grow faster than characteristic frequency, if there is metastable growth at all. As the simulation proceeds to further to $t=1.368102 \times$ $10^{-5}$, the peak becomes more monotonically decreasing, which is the proof of metastable growth. Figure 5.1-32 shows relative concentration of configuration at $t=2.247859 \times 10^{-6}$ after applying the band-pass filter with frequency range indicated above each subplot. First, all of high concentration peaks at the boundary correspond to frequency higher than 7 , as only the band-pass filter higher than 7 shows significantly higher peaks mentioned. Thus, again, the 
droplets at the boundary have the energy advantage, therefore, they may adopts high frequency. While absolute concentration as shown in Figure 5.1-28 shows that some peaks are more dominant than the other, after band-pass filtering with $\left(r_{1}, r_{2}\right)=(3,5)$ and $(5,7)$, the all droplets seem to have relatively equal contrast and equally spread out. This shows that the structure factor at low frequencies have been rising surely. That is why many droplets did not disappear in contour plot of Figure 5.1-29. Because structure factor with high frequencies are quickly eliminated, especially when the droplets are entirely within the domain, not at the boundary, the collapse of many droplets as absolute concentration is seen in Figure 5.1-28. When observing band-pass filter of $\left(r_{1}, r_{2}\right)=(5,7)$, the highly concentrated droplets are the droplets that have enlarged by disappear-and-enlarge mechanism over time, as shown in Figure 5.1-28. Thus, this further strengthens discussion regarding high structure factor at $f=4$. Relative energy diagram in Figure 5.1-34 helps to break down the process into several step. First, there is a quick drop in relative energy until about $t=1.0 \times 10^{-6}$. This is when most of the droplets are shrinking rapidly. But the rate seems to be slower from this point as few droplets will persist longer than the most. It will then enter a relatively stationary state in which relative energy is about zero. From the beginning up to this point, all of the droplets, including the disappearing ones, have been conforming to favorable morphology, e.g. the configuration with broad, monotonically decreasing structure factor with the frequency. Once it has reached the negative relative energy value, the remaining 6 droplets rise closer to equilibrium values. This process is similar to the case in which the second metastable quench is started with relative energy of zero or lower. In this process, the droplets at the boundary will rise faster because of energy advantage. 

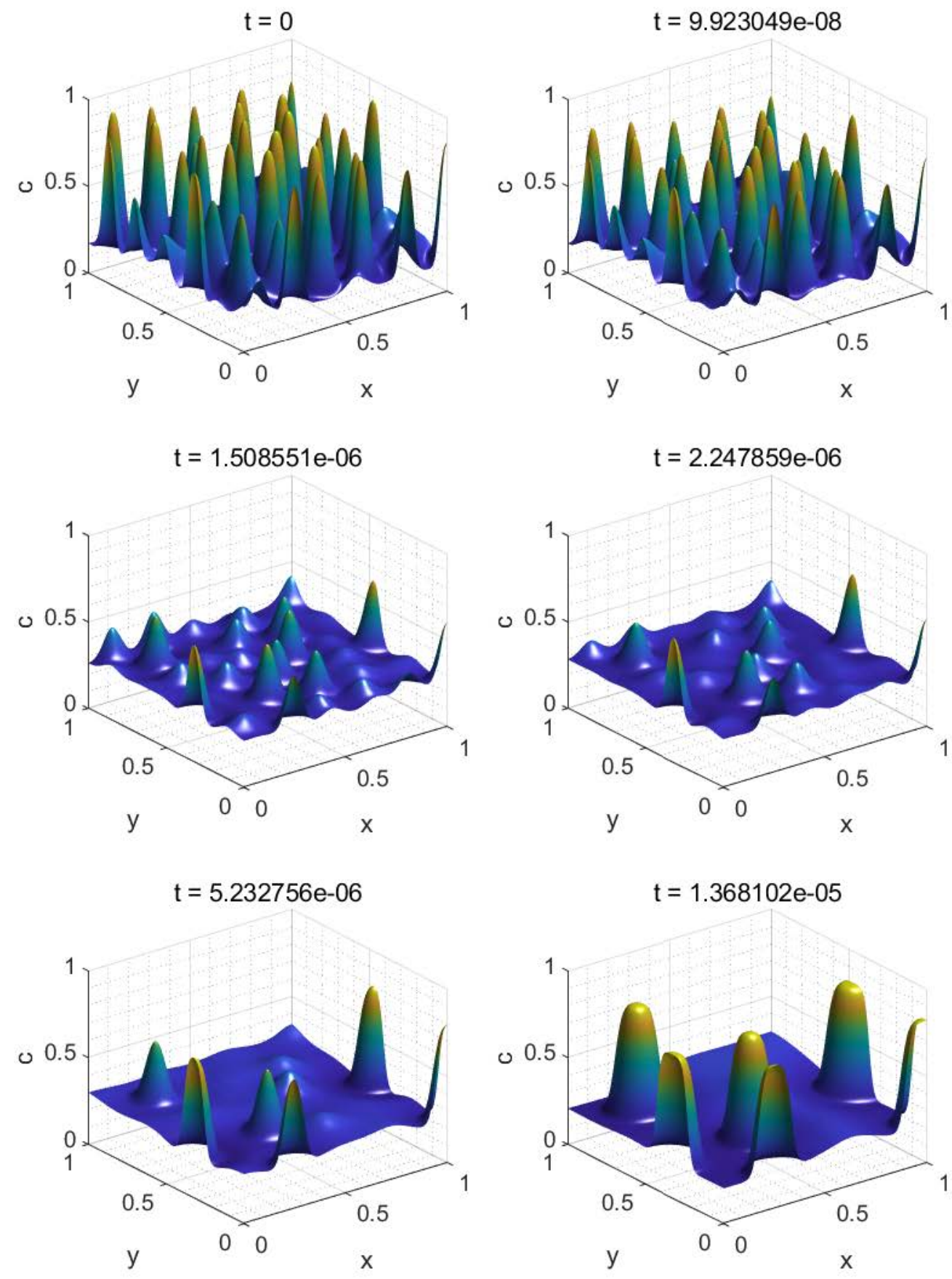

Figure 5.1-28 Concentration profile of metastable second quench (Case 5.1.2.D) 

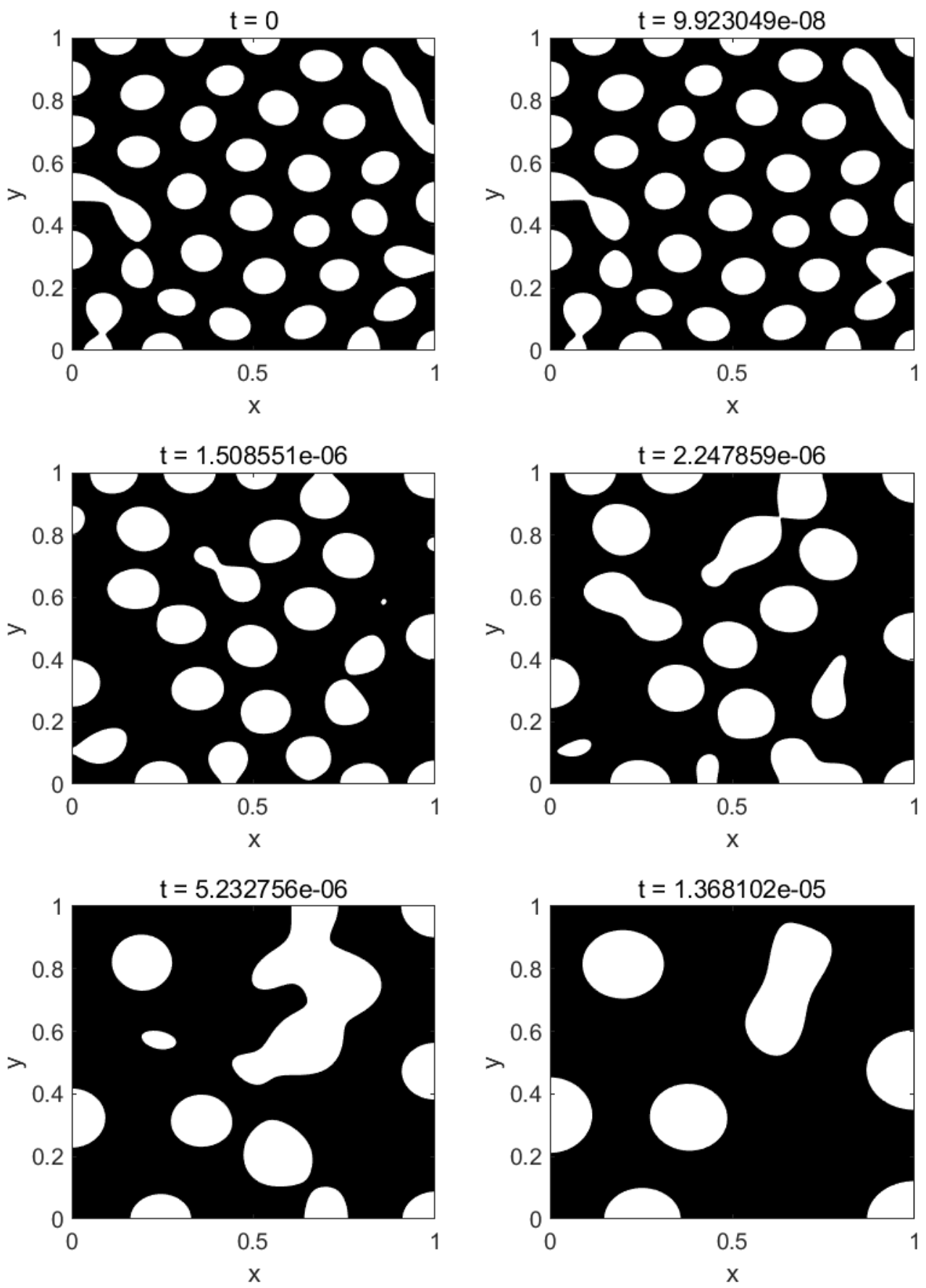

Figure 5.1-29 Contour plots of the same simulation as in Figure 5.1-28 (Case 5.1.2.D) 

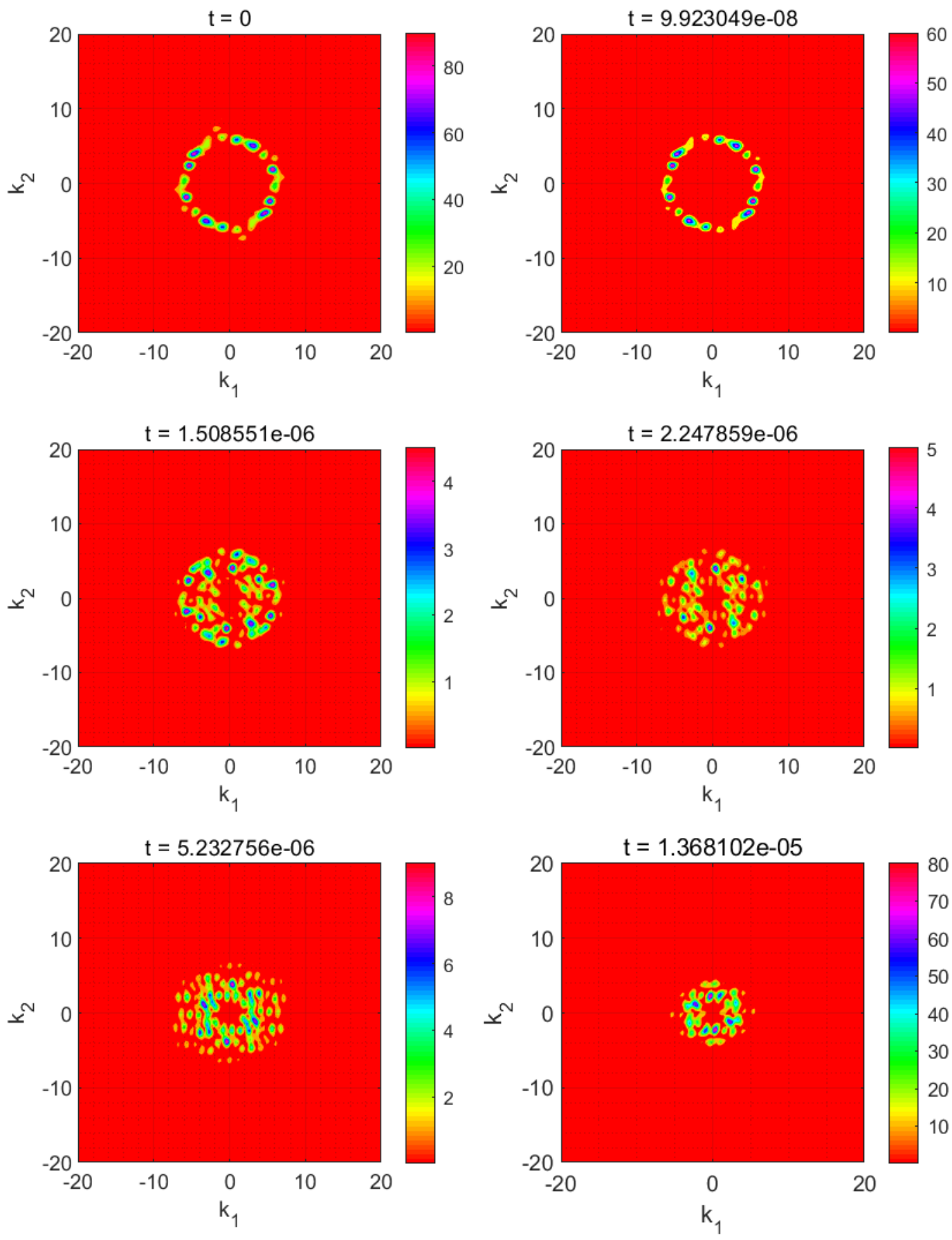

Figure 5.1-30 Amplitude spectrum of the same simulation as in Figure 5.1-28 (Case 5.1.2.D) 

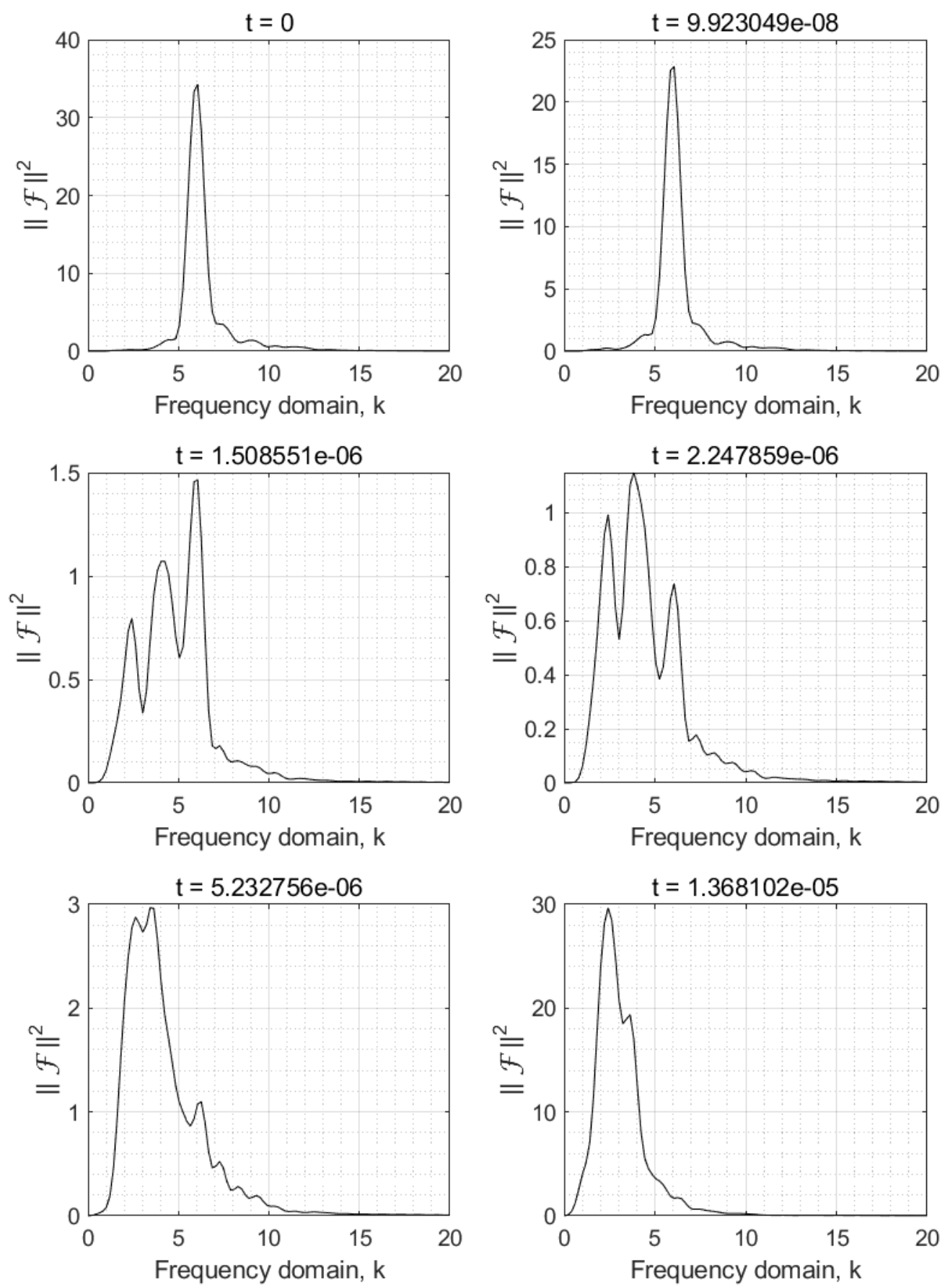

Figure 5.1-31 Circularly averaged amplitude spectrum of the same simulation as in Figure 5.1-28 (Case 5.1.2.D) 


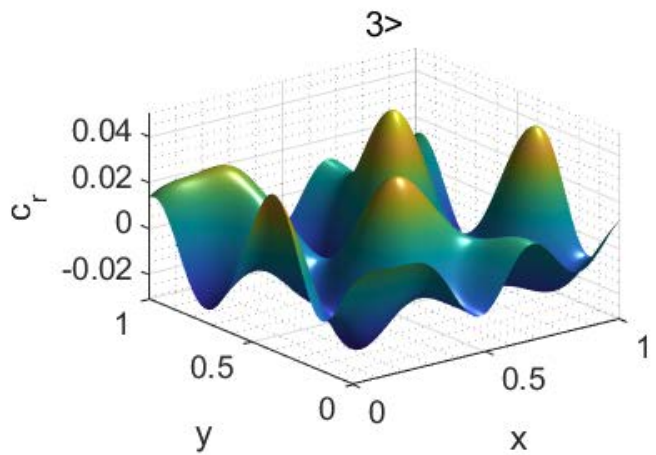

$(5,7)$

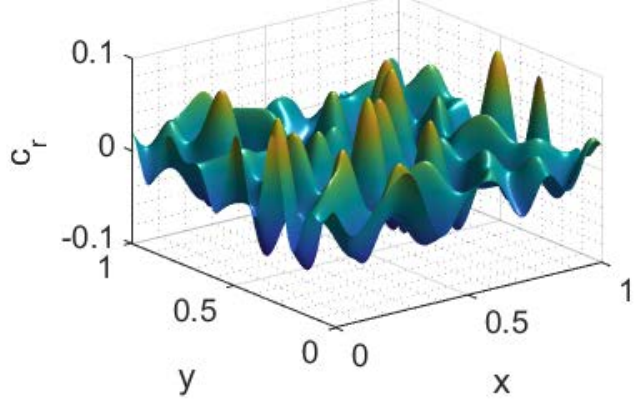

$(3,5)$

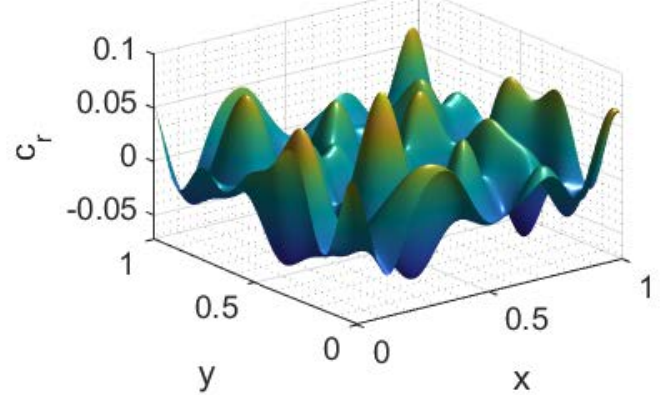

$7<$

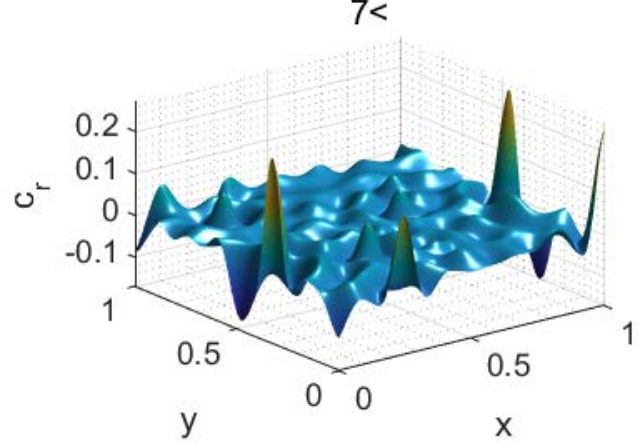

Figure 5.1-32 Band-pass filters were applied at $t=2.247859 \times 10^{-6}$ (Case 5.1.2.D)

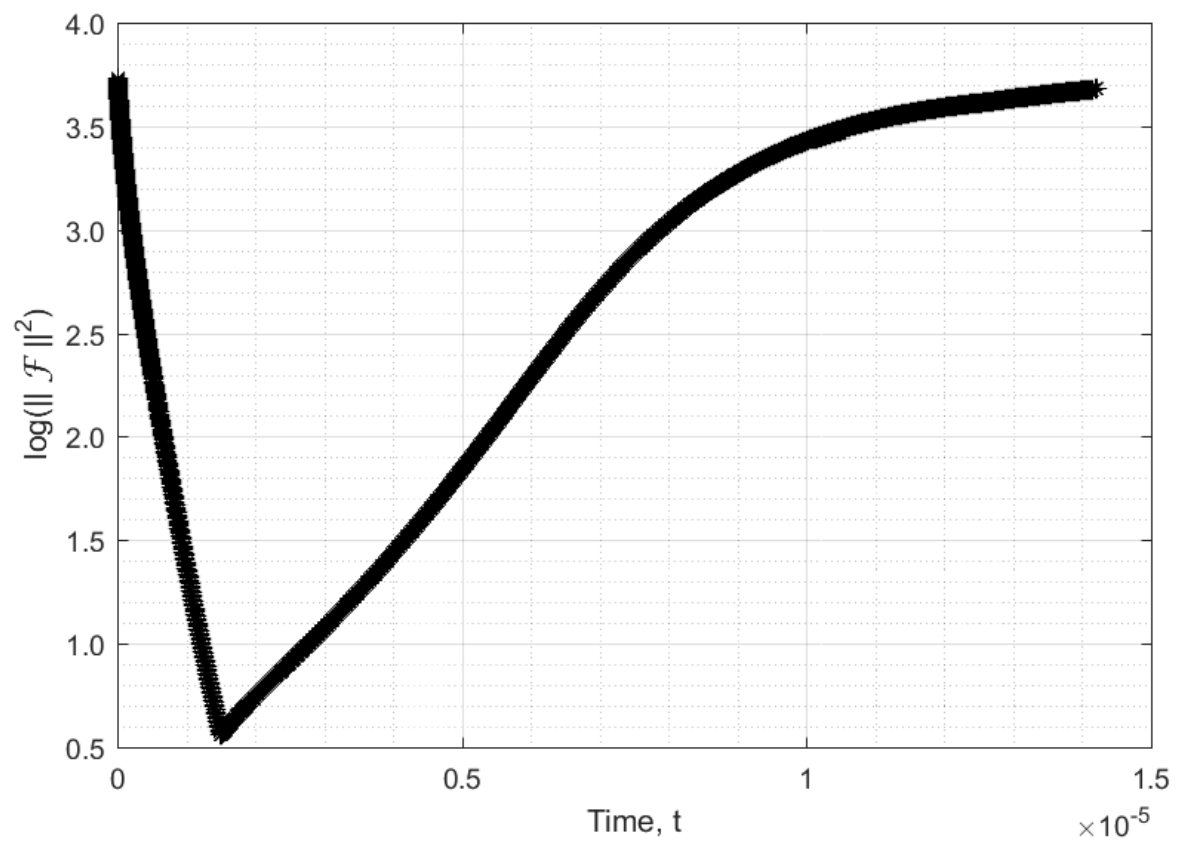

Figure 5.1-33 Log plot of maximum amplitude of the same simulation as in Figure 5.1-28 (Case 5.1.2.D) 


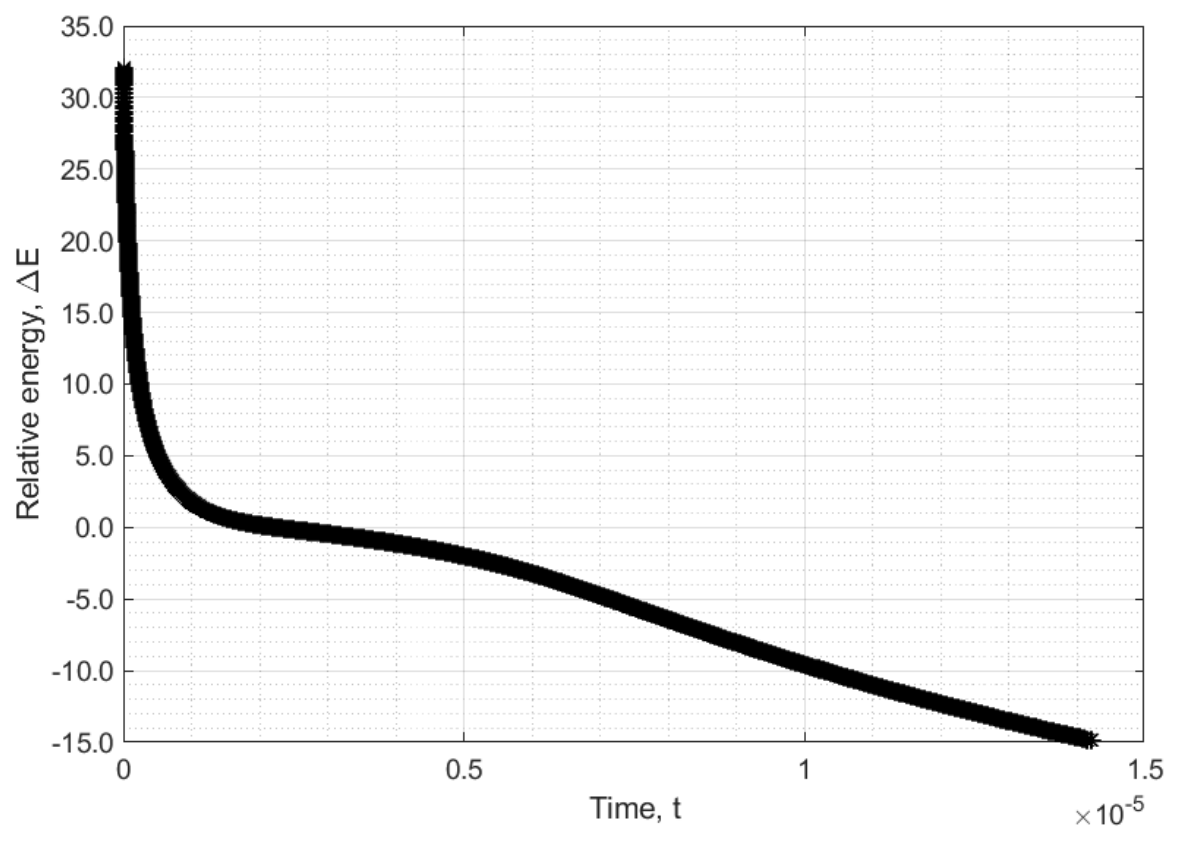

Figure 5.1-34 Relative energy of the same simulation as in Figure 5.1-28 (Case 5.1.2.D)

Double quench in metastable region will, in other cases, force the solution to go back to uniform state. This is expected the higher the starting relative energy is, with all other variables constant. This, then, is expected the further the second quench is away from spinodal line and close it is to binodal line. This time, the very same configuration as before is taken, i.e. $D=$ 10000, $c_{\text {ave }}=0.3, T=0.3, n_{1}=n_{2}=1$ (Case 5.1.2.C), with time frame $t=5.318622 \times 10^{-6}$, then the temperature is quickly raised to $T=0.36$ (Case 5.1.2.E). The overall process is shown in Figure 5.1-35. All of the droplets disappear eventually by the time $t=1.253219 \times 10^{-5}$. However, not all of the droplets seem to appear at the same rate. Initially, some droplets disappear and at the same time enlarging the width of the neighboring droplets. This mechanism is very much similar to the late stage of typical spinodal decomposition. This process takes place until at least $t=8.121522 \times 10^{-7}$. Once all of the droplets are brought close to average concentration, the merging of the droplets occur, creating co-continuous structures like the one seen at $t=1.607217 \times 10^{-6}$. Figure 5.1-37 shows the structure factor of the configuration. It can be seen that, as the time proceeds, the characteristic ring lowers its amplitude, exposing the structures at the lower frequency. In Figure 5.1-38, there are 3 
apparent peaks at $t=8.121522 \times 10^{-7}$. They are located at about $f=2.1, f=4.1$, and $f=$ 6 , in increasing order of the amplitude. In order to explain what each of these peaks means, band-pass filters are applied at configuration $t=8.121522 \times 10^{-7}$ and shown in Figure 5.1-39. It can be seen that, high frequency structures, $f>7$, corresponds to droplets at the boundaries. Because there is no interfacial energy between the solution and boundaries in present model, these droplets have less interfacial energy, therefore they collapse slower than the others. The filter $\left(r_{1}, r_{2}\right)=(5,7)$ shows that the structures are more concentrated within the internal region of the domain. This is the initially established characteristic structures that still remain and is dominant at this time frame. Of the structures with the frequencies below this point seem to spread out evenly. The peak at $f=4.1$ is possible the result of the droplets' disappearance and enlargement of neighboring droplets. The peak at $f=2.1$ shows metastable growth. To break down the behavior of all of these peaks over time, Figure 5.1-40 shows the log of structure factor at the frequencies mentioned. As can be seen here, the peaks at $f=4.1$ and $f=6$ monotonically decrease at the same time, but at the different rate. The collapsing of $f=6$ is explained as it is the most dominant structure factor initially, thus it has the highest interfacial energy. While the structure at $f=4.1$ takes its shape as the droplets disappear and enlarge in width, the amplitude still decrease due to its unfavorable interfacial energy. Most notably, there is rise in structure factor at $f=2.1$ initially. This shows that metastable phase separation was occurring in the lower range of frequency domain. However, eventually these also collapse because of high interfacial energy. Therefore, there are two factors at play in this scenario: one is metastable growth, in which, the structures develop in such way that the structure factor is monotonically decreasing in frequency. Another is by elimination of unfavorable energy due to fluctuation, especially by interfacial energy. This way, the dominant peaks at higher frequency is eliminated the fastest. For double quench from unstable region into metastable region, it is the characteristic structure factor. As this peak collapses, the next dominant peak at lower frequency is also dragged on and collapse, but at the slower rate, and so on, making it look like a domino effect. Eventually, these collapsing reach the low frequency structures that are evolving by NG, and cause structures to collapse to uniform state. The result is a monotonically decreasing structure factor in frequency domain. The ascending order of three peaks at $t=8.121522 \times 10^{-7}$ is completely switched by $t=$ 
$1.607217 \times 10^{-6}$, then yields monotonically decreasing structure factor at $t=1.253219 \times$ $10^{-5}$. Indeed, the solution returning to uniform state means that any fluctuation within it is eliminated, so the result is the only peak at very low frequency. Figure 5.1-41 shows the log plot of maximum structure factor. There are "kink" or sharply bent structure at about $t=1.0 \times$ $10^{-6}, t=3.0 \times 10^{-6}$ and $t=7.4 \times 10^{-6}$. These are when the most dominant peaks have decreased lower than the next dominant peaks at lower frequencies. Note that the curve becomes relatively less and less steep after each "kink" because the structures at lower frequencies collapse slower. Figure 5.1-42 shows relative energy's change throughout the entire time. Indeed, the energy decreases quickly initially, then approaches the stationary state around zero but never reaches it. The decrease in the energy is smooth. Quick drop in relative energy is the time period in which the solution quickly returns to uniform state. 

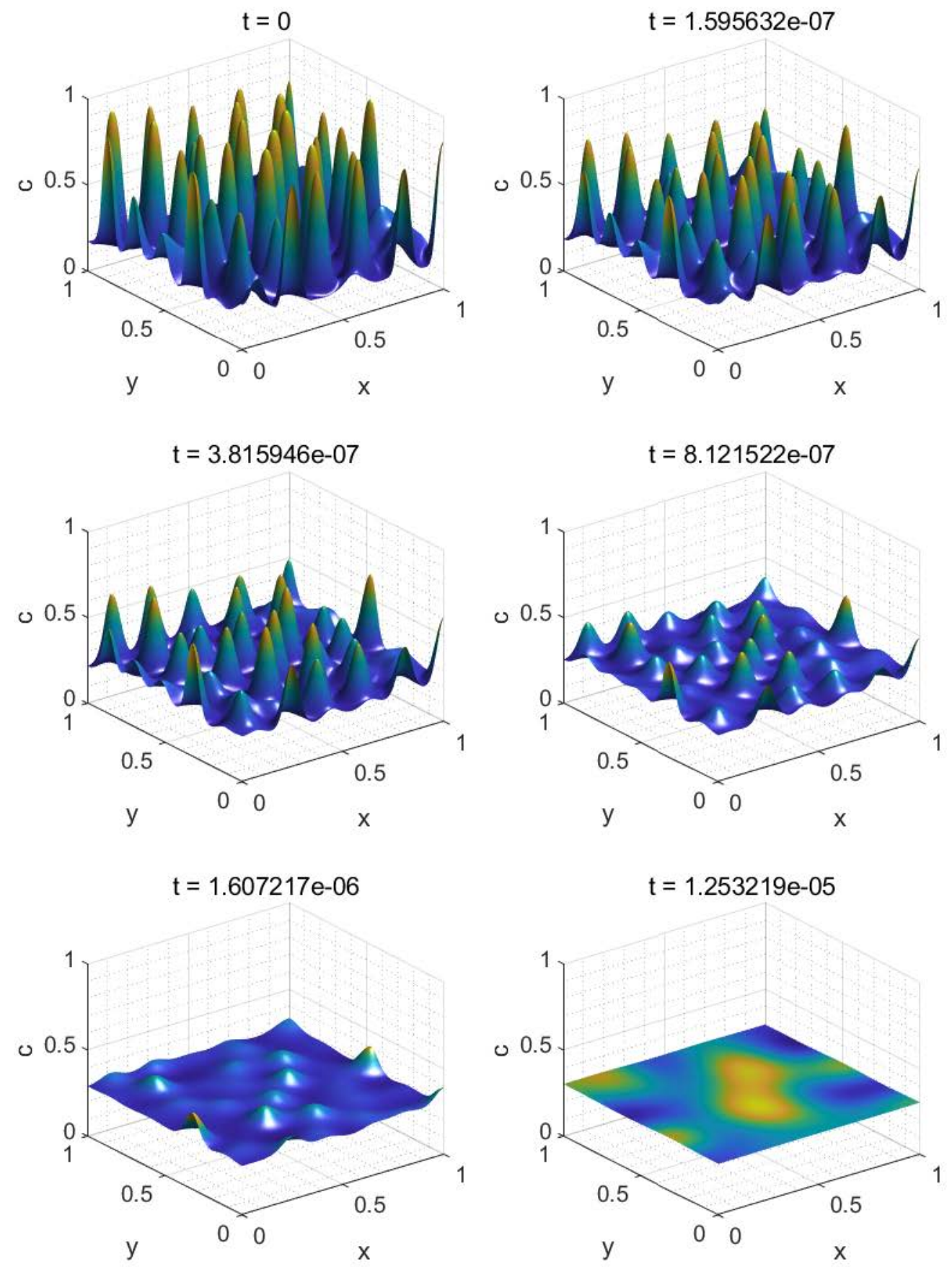

Figure 5.1-35 Second metastable quench with 10000, $c_{\text {ave }}=0.3, T=0.36, n_{1}=n_{2}=1$ where $c_{o}$ is taken from configuration obtained in Case 5.1.2.C at $t=5.318622 \times 10^{-6}$ (Case 5.1.2.E) 

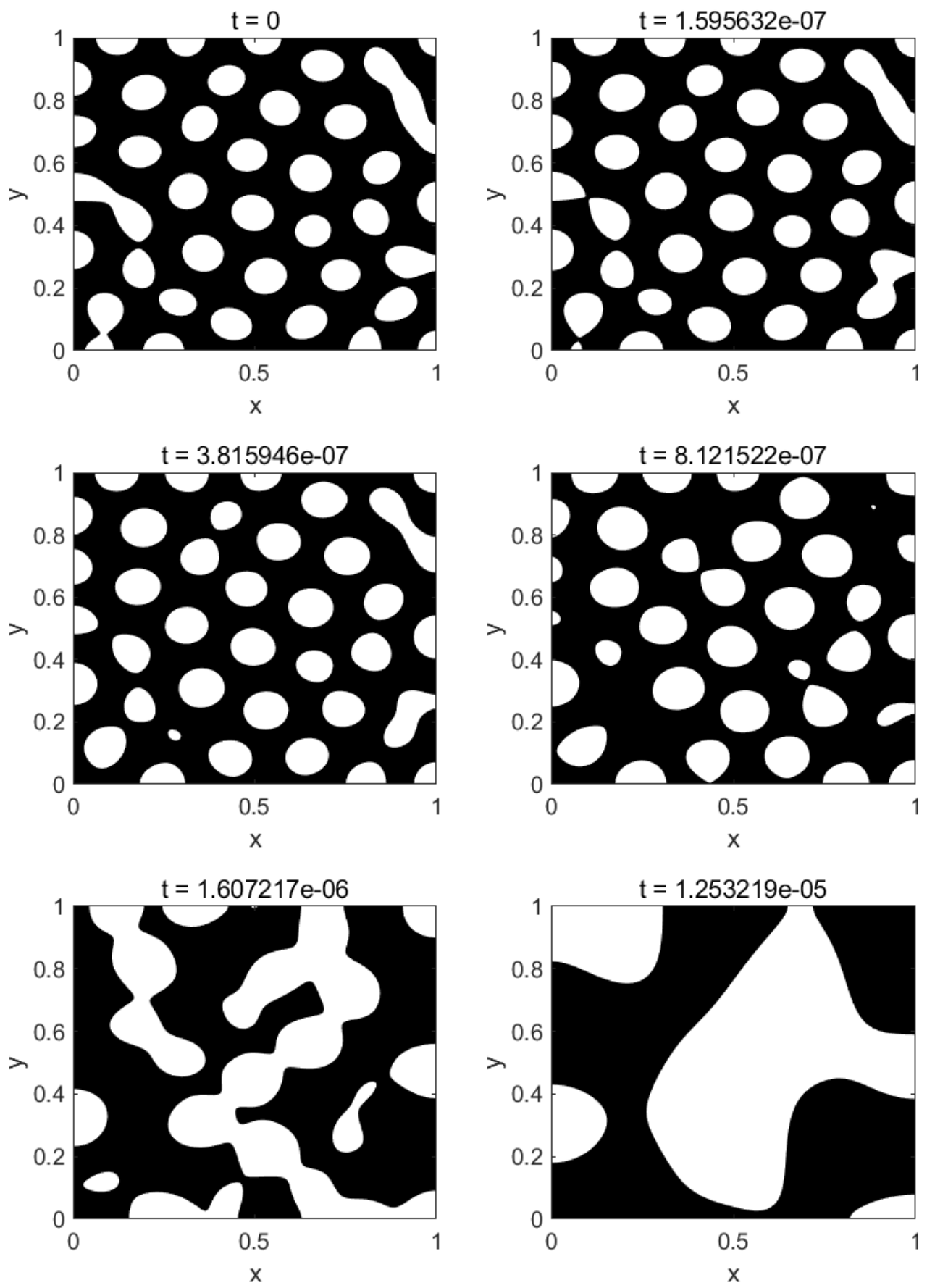

Figure 5.1-36 Contour plots of the same simulation as in Figure 5.1-35 (Case 5.1.2.E) 

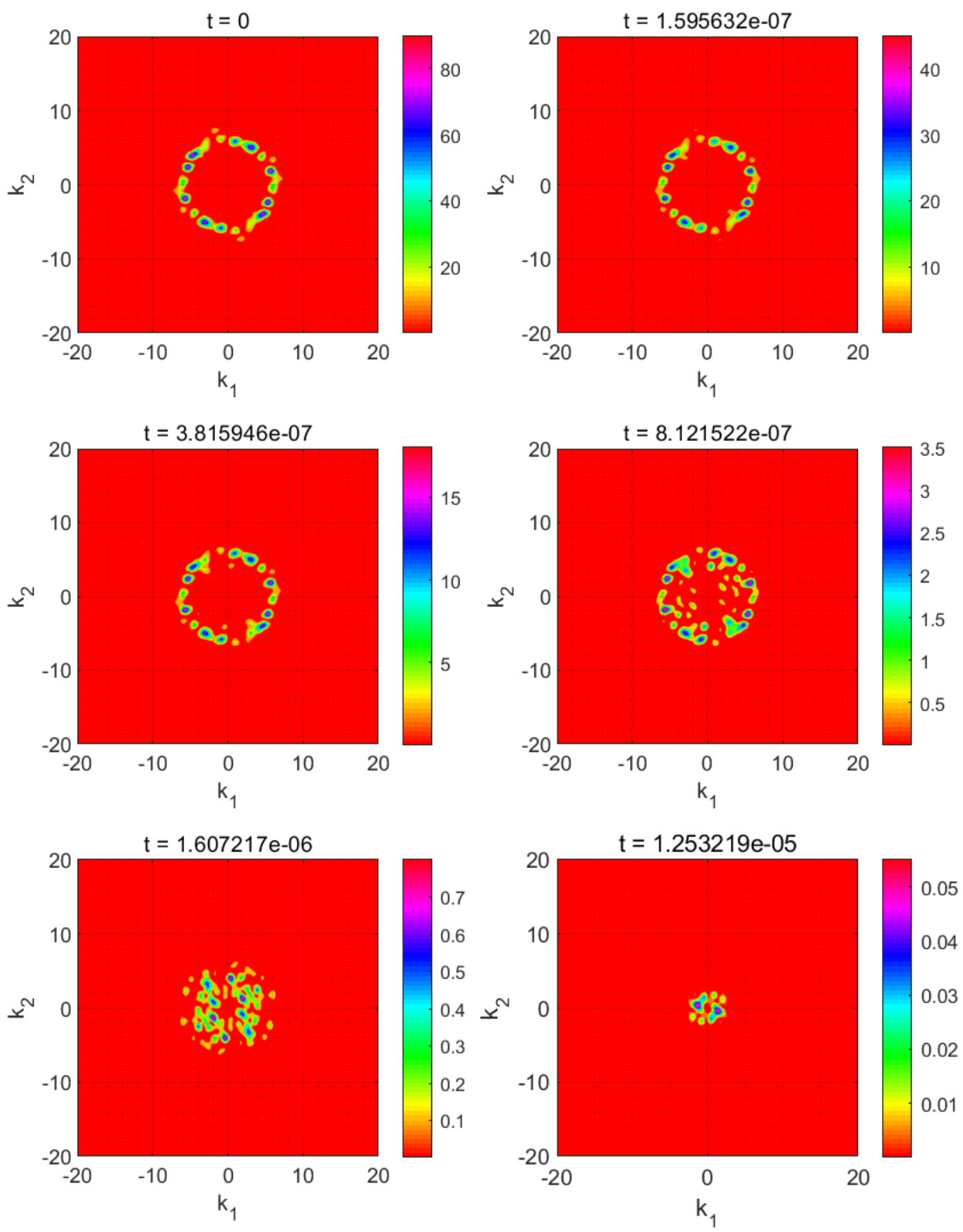

Figure 5.1-37 Amplitude spectrum of the same simulation as in Figure 5.1-35 (Case 5.1.2.E) 

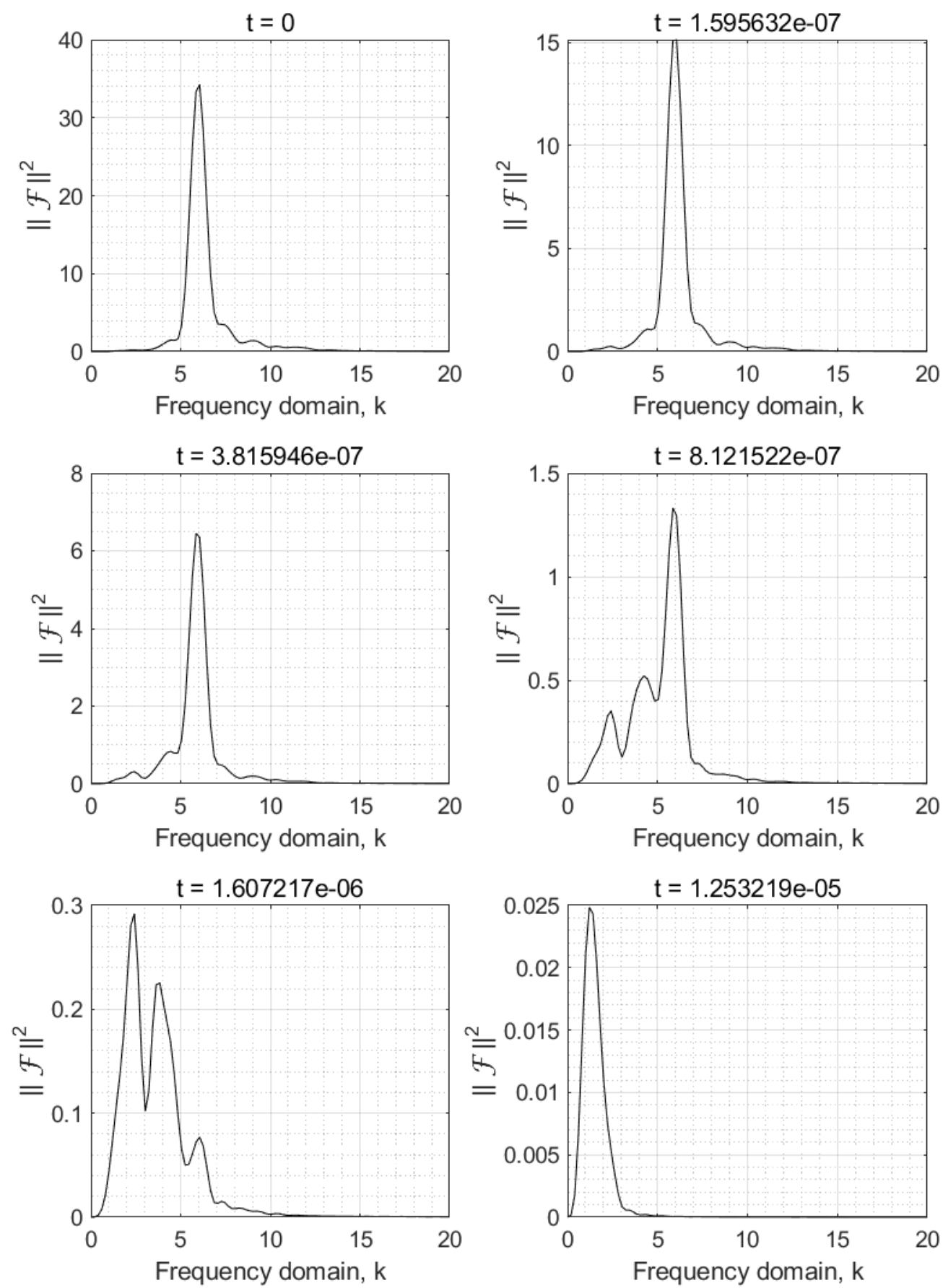

Figure 5.1-38 Circularly averaged amplitude spectrum of the same simulation as in Figure 5.1-35 (Case 5.1.2.E) 


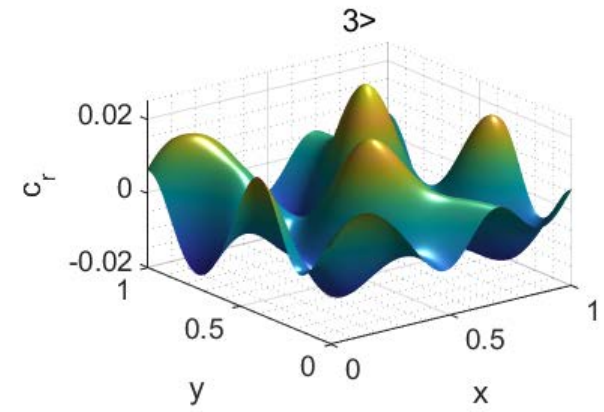

$(5,7)$

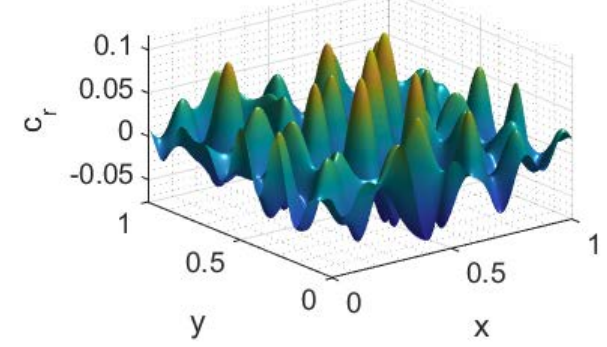

$(3,5)$

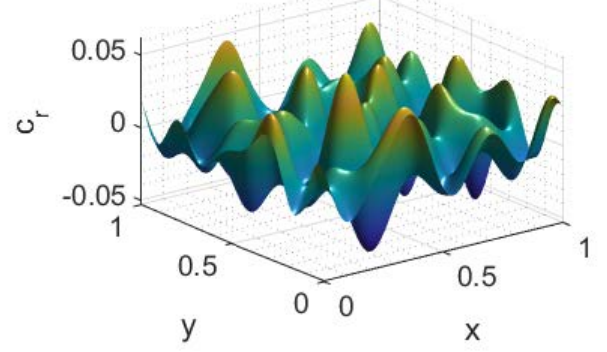

$7<$

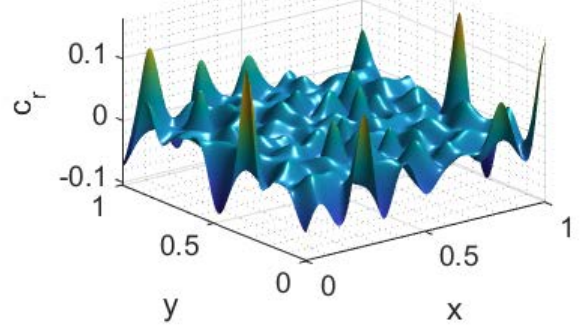

Figure 5.1-39 Band-pass filters applied to configuration at $t=8.121522 \times 10^{-7}$ (Case 5.1.2.E)

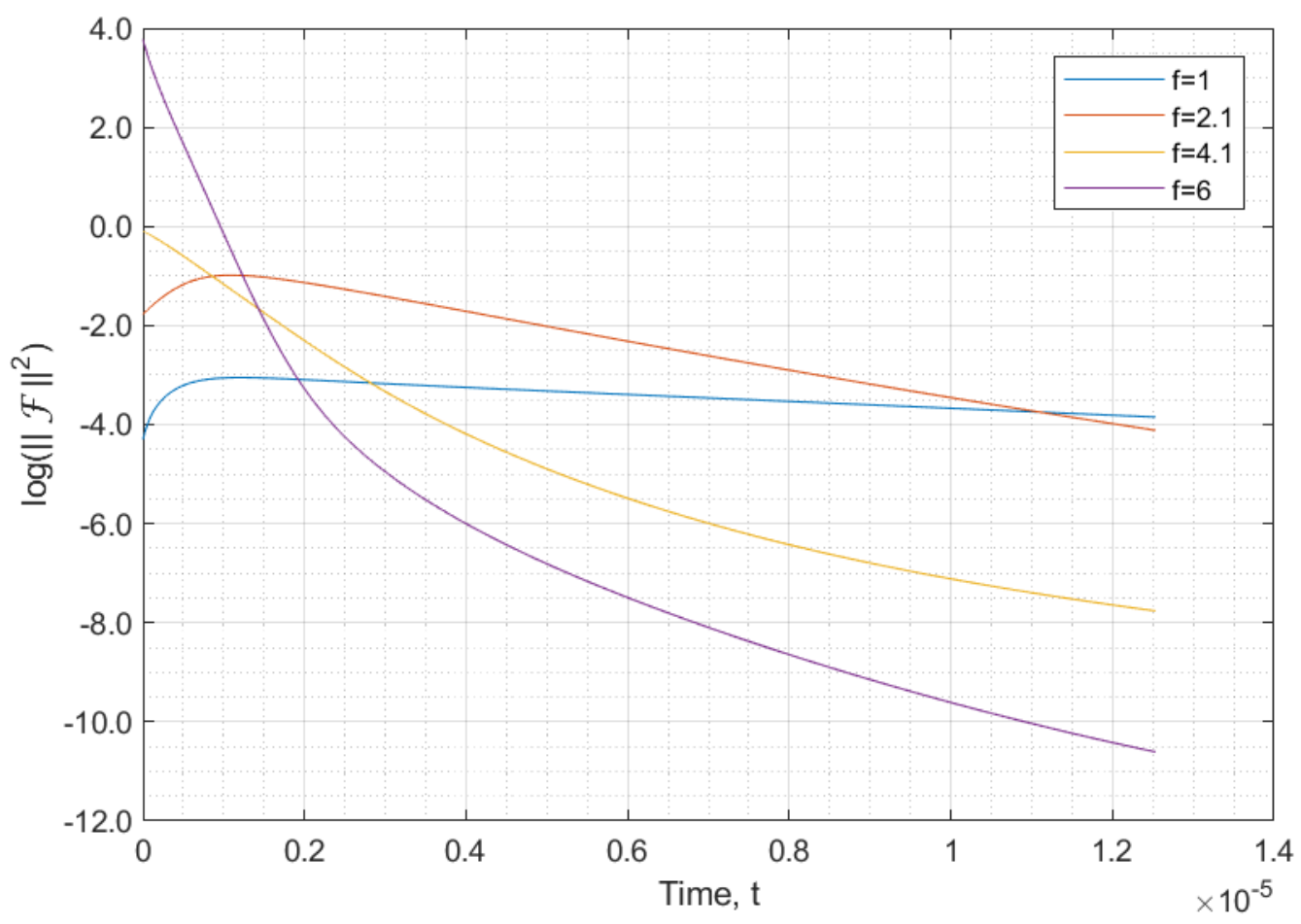

Figure 5.1-40 Log plot of amplitudes of specific frequencies of the same simulation as in Figure 5.1-35 (Case 5.1.2.E) 


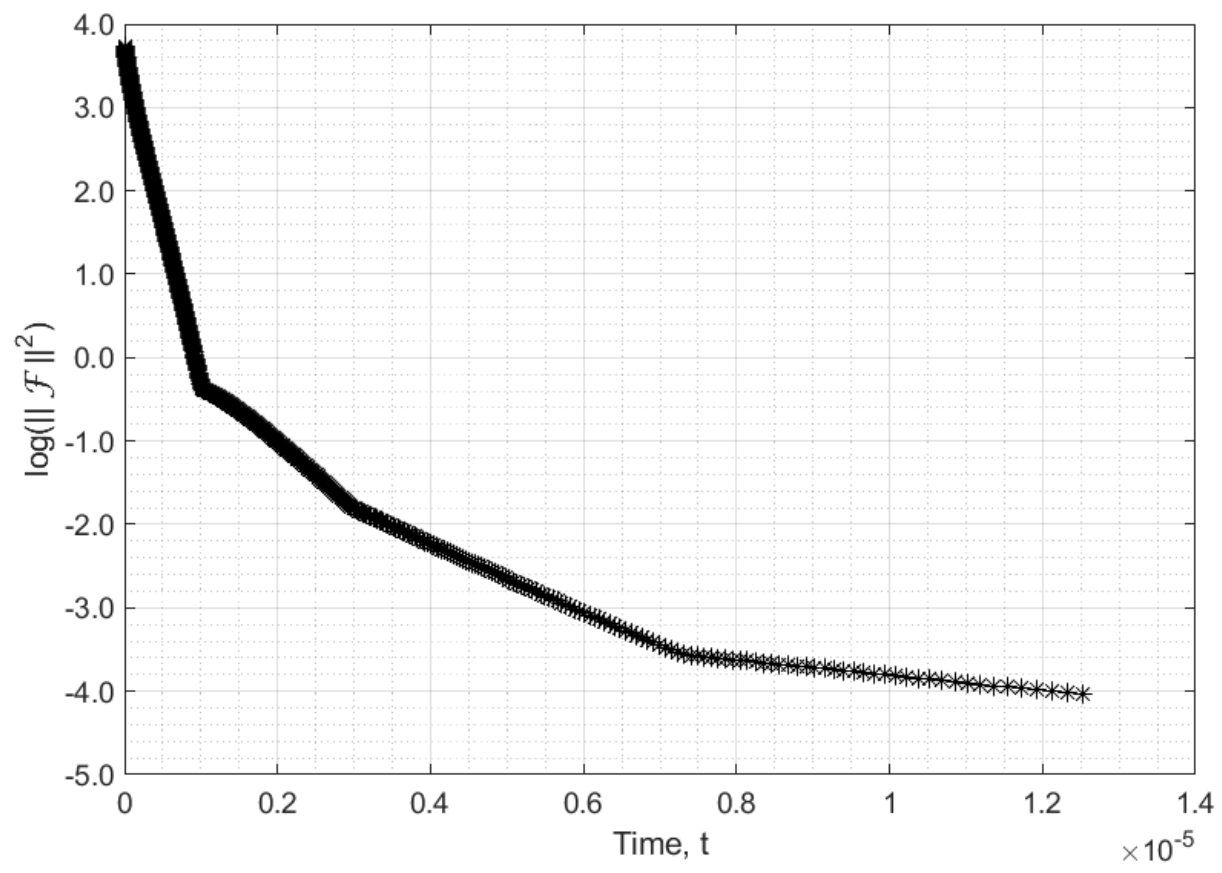

Figure 5.1-41 Log plot of maximum amplitude of the same simulation as in Figure 5.1-35 (Case 5.1.2.E)

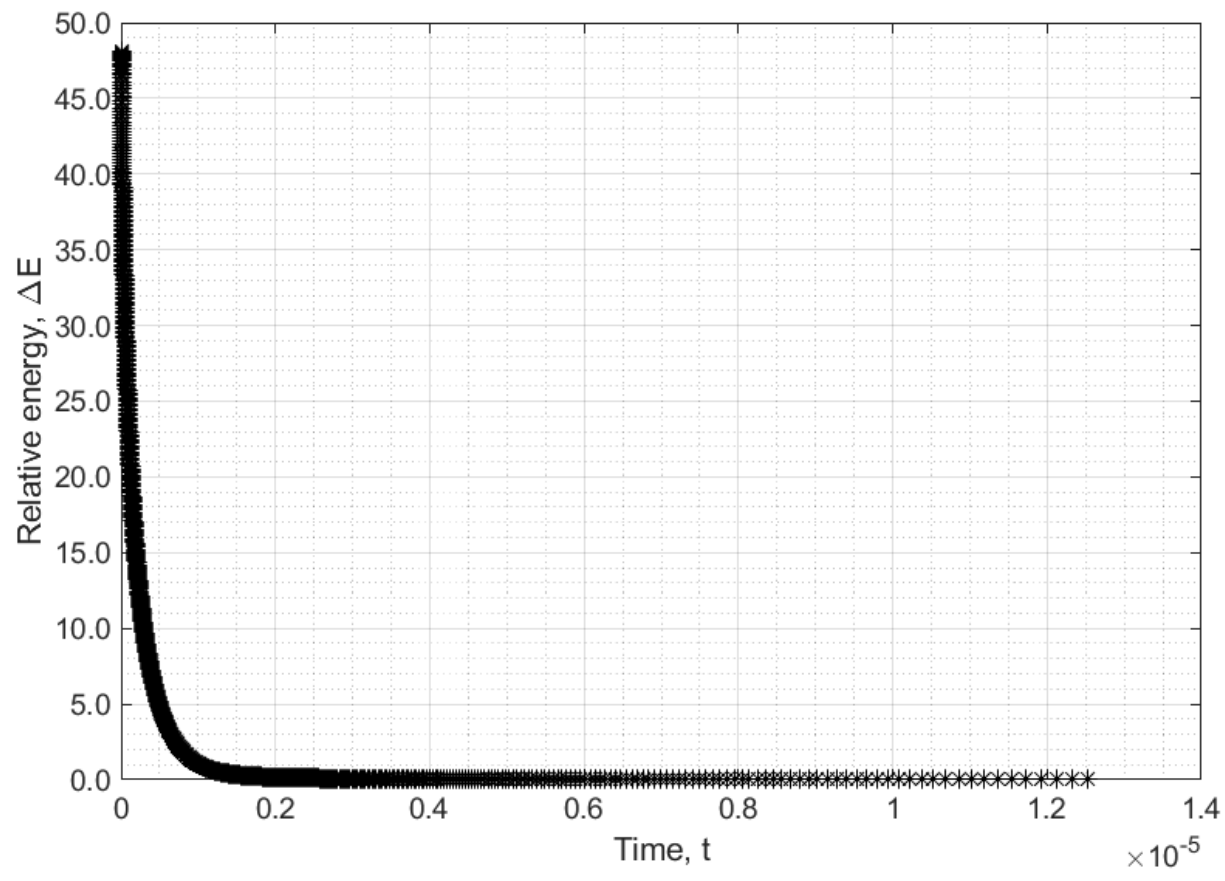

Figure 5.1-42 Relative energy of the same simulation as in Figure 5.1-35 (Case 5.1.2.E) 


\subsection{Anisotropic quenching}

\subsubsection{Temperature gradient}

Anisotropic quenching has been of great interest research for some time. Anisotropic quench is when the quenching parameters vary across the domain. The result is normally a non-uniform morphology if the condition is right. Since for present model, generally, the deeper the quench is, or the lower it is from spinodal line if all other parameters are constant, the higher the characteristic frequency is. Thus, if the temperature is varied across the domain, one might simply expect smaller droplets or interconnected structures on the deeper quench side if the temperature is varied across the domain. However, the process can be more complicated because some regions will phase-separate faster than the other regions, leading to special morphology due to already-established configuration, as seen in double quench region. Also, this time, there is a diffusion in the direction of the temperature gradient immediately from the start, because free energy model, Flory-Huggins, is dependent on the temperature. Thus, if all other parameters constant, the difference in the temperature can cause imbalance in potential difference across domain, leading to the flow due to energy imbalance alone. This effect is especially intensified if the initial noise is small, because the solution's phase separation depends less on the local concentration fluctuation, thereby flow solely in the direction of temperature gradient results. One must also be careful in choosing the type of gradient one chooses and be aware of the behavior that it may cause. For example, if linear temperature gradient is chosen, then at the uniform state, there is a potential difference immediately next to the boundaries in the direction of the gradient. This causes the flux to fall immediately toward the wall if the potential difference is lower in direction normal outward of the boundary, of away from the wall if the potential difference is higher in the direction normal outward of the wall. Because of no mass flux boundary condition, component will either get squeezed towards the wall or repelled from the wall, leading to a concentration fluctuation that takes shape according to the wall. For $2-d$ square domain, a lamellar structure results. Since spinodal decomposition is sensitive to initially established configuration, one may expect lamellar 
structures to propagate away from the walls and into the domain. Again, this phenomenon is more intensified with smaller noise.

Because in this thesis, the behavior of phase separation within the internal region of the domain is of more concern than the influence of the boundary, initial fluctuation's noise is raised to $\left\|\eta_{o}\right\|_{\max }=1.0 \times 10^{-3}$ and a temperature gradient that has zero derivative at the direction normal outward at all boundaries is chosen. More specifically, temperature gradient that has the form of third degree polynomial is chosen and it satisfied the following

$$
\begin{gathered}
\left.T\right|_{x=0}=T_{1} \\
\left.T\right|_{x=1}=T_{2} \\
\left.\nabla T\right|_{x=0}=\left.\nabla T\right|_{x=1}=\overrightarrow{0}
\end{gathered}
$$

However, third degree polynomial chosen here is still not perfect for all experimental observations as it may cause complication in the amount of mass flux, creating imbalance in the flow with direction of temperature gradient. Nonetheless, it still helps to shed some light into the behavior of anisotropic quenching and fulfill the purpose of this essay.

While multiple researches have been done on anisotropic quenching using non-uniform temperature, special attention is paid to Tran-Cong-Miyata and Okinaka's research [27] [28]. In their experiment, they used poly(2-chlorostyrene)/poly(vinyl methyl ether) blend with two heating adjusters at two ends. By using them, temperature gradient was applied gradually with time. The result was a spatially graded morphology in which the deeper quench region starts to show droplet structures because they've come to late stage earlier, while co-continuous structures are seen in the middle region of the domain as phase separation is just starting. For simulations, the temperature is not varied with time and no region will be exposed to stable region of phase diagram. Nonetheless, it still produces a result that closely mimic experimental observations.

First, the anisotropic quench with $D=100000, c_{\text {ave }}=0.3, n_{1}=n_{2}=1$ and temperature varies with $T_{1}=0.34$ with $T_{2}=0.30$ (Case 5.2.1.A) is shown in Figure 5.2-1. Concentration profile in Figure 5.2-1 shows that droplets start appearing from deeper side of the quench. By 
the time $t=5.065364 \times 10^{-9}$, Figure 5.2-2 shows that the size of co-continuous structures already varies across the domain in x-direction. At time $t=3.539219 \times 10^{-8}$, spatially graded structure has formed: the droplet structure on the deeper quench side because they have are entering the late stage, while the remaining portion of the domain has co-continuous structures because they are still forming into favorable morphology before fast phase separation begins. At this time, the sizes of co-continuous structures still varies in $\mathrm{x}$-direction with smaller sizes on right. Note that, at this time, most of shallow quench region is above average concentration, so it is not possible to view evolving co-continuous structures in such shallow region. Also note that this is due to uniaxial diffusion, which suggests that the diffusion is occurring along concentration gradient. This is the region where the effect of initial concentration fluctuation is inferior compared to potential difference created solely by the temperature difference. Over time, the region with droplet structures propagate towards shallower region, creating more droplets as shown until $t=1.046384 \times 10^{-7}$. Because the droplets in the deeper quench region is already in late stage, some droplets have already started to shrink and disappear, while the neighboring droplets enlarge. In these stages, new droplets seem to arise from cocontinuous structures that is aligned vertically in y-direction. In these regions where new droplets are formed, there seem to be a structure that has already been established by potential difference due to temperature gradient and it is more profound until this point. Also, the vertical co-continuous structure is explained by this: the already phase separated structures adjacent to newly evolving structures has acted as the "initiator" for the emerging droplets, just like the mechanism that helped to form concentric pattern in double quench study in previous section. With well phase-separated region in adjacency as initiator, which is nicely aligned in vertical line, the newly emerging co-continuous structures align according to it. The discussion so far is rather qualitative. Figure $5.2-3$ shows structure factor of this simulation. At $=$ $5.065364 \times 10^{-9}$, asymmetric peak at about $k_{1}= \pm 0.5$ and $k_{2}=0$ can be observed. Since these peaks appear only when $k_{2}=0$, there is a structure that varies only uniaxially. Figure 5.2-5 shows the configurations after band-pass filters have been applied. The structures with $f<2$ seems to vary in only $x$ direction and manifests sinusoidal-like shape. Structures with high frequency $f>15$ shows that there is growth in deeper quench region. All other filters in between show the droplets that spread out evenly. Thus, there are two distinct driving force 
here in phase separation: one is by initial concentration fluctuation. Here, they follow the simply rule that, the region with deeper quench will see higher frequency droplets first. At the low frequency, at structure develop uniaxially because of uniaxial temperature gradient. The concentration flows simply by potential difference created as a result varying temperature. This effect is seen everywhere across the domain. Since the driving force of spinodal decomposition by initial concentration fluctuation is lower in shallower region, most of this region has concentration that is higher than the average one. The peaks at $k_{1}= \pm 0.5$ and $k_{2}=0$ persisted and grow throughout the entire simulation time. However, in concentration profile, they are visible only when the mixture is re-organizing into favorable shapes prior to fast phase separation, which is slower in shallower region. Over time, spinodal decomposition due to initial concentration fluctuation prevails. At time $t=3.539219 \times 10^{-8}$, a ring structure is seen in Figure 5.2-3. This represents current dominating structures. In closer look, every point on the arc of the ring seems to be "stretched" in the direction of $k_{1}$. In order to understand this, bandpass filters are applied for this configuration and shown as contour plots in Figure 5.2-6. First of all, macro-structures of low frequency, $f<1$ is uniaxial throughout the entire domain, and has been evolving slowly. On the other hand, large frequency structure with $f>18.5$ are concentrated mostly on deeper quench side, in the droplets forms. In the intermediate range with $\left(r_{1}, r_{2}\right)=(17,18.5)$, the structure has evolved mostly on the deepest quench side, and second mostly on the shallowest quench side. It is least present within the intermediate range. This is because the temperature gradient has "pushed" component 1 towards shallower quench region. Once component 1 has become "trapped" in this shallow quench region causing uniaxial fluctuation, because of interfacial energy advantage, this ultimately leads to earlier rise in the droplets. In deeper quench region, while high frequency structures take the shape of the circular droplets, structures of the intermediate frequency seems to be stretched along $\mathrm{x}$ direction due to uniaxial flow in temperature gradient. In fact, there is a slight leftward shift of the droplets at this stage, causing these structures look a little bit stretched. Eventually, the ring becomes less "stretchy" in x-direction, and become somewhat more defined at time $t=$ $1.046384 \times 10^{-7}$. At this stage, many droplets have formed and the uniaxial flow by temperature gradient has become less significant. Figure 5.2-10 shows the log plot of maximum structure factor over time. Several kinks are present. These are when the maximum structure 
factor is surpassed by that of lower frequency. Figure 5.2-11 shows the change in relative energy over time. A quick sudden drop in relative energy does not start until at about $t=3.0 \times$ $10^{-8}$. Until this time, low frequency structures are forming significantly. After this time, the phases reach rapidly to their equilibrium values. To better identify structural variation across the domain quantitatively, Figure 5.1-7, Figure 5.2-8, and Figure 5.2-9 show the variance of the concentration profile along the vertical line from $y=0$ to $y=1$ for across the values of $x$, for $\mathrm{t}=5.065364 \times 10^{-9}, t=3.539219 \times 10^{-8}$, and $t=1.046384 \times 10^{-7}$ respectively. Recall that the variance is related to the measure of phase separation. At $t=5.065364 \times 10^{-9}$, only the variance for the filter with highest frequency, $r>15$, seems to vary across the domain most significantly. In particular, it increases with deeper quench. Thus, high frequency structures are more abundant in deeper quench region. At $t=3.539219 \times 10^{-8}$, the regions too close to $x=0$ and $x=1$ are disregarded, high frequency structures with $r>18.5$ is spread out without pattern from approximately $x=0.85$ to $x=0.95$. The spread is even wider at $\mathrm{t}=1.046384 \times 10^{-7}$ from approximately $x=0.5$ to $x=0.95$. However, at this time, the lower frequency structures with $\left(r_{1}, r_{2}\right)=(2,10)$ dominate in deeper quench regions. Thus, a "catch-up effect" is observed, where the structures of newly phase-separated regions become similar to those in the deeper quench regions, then as they proceed further into slower late stage, these structures become more synchronized and coarsen together. 

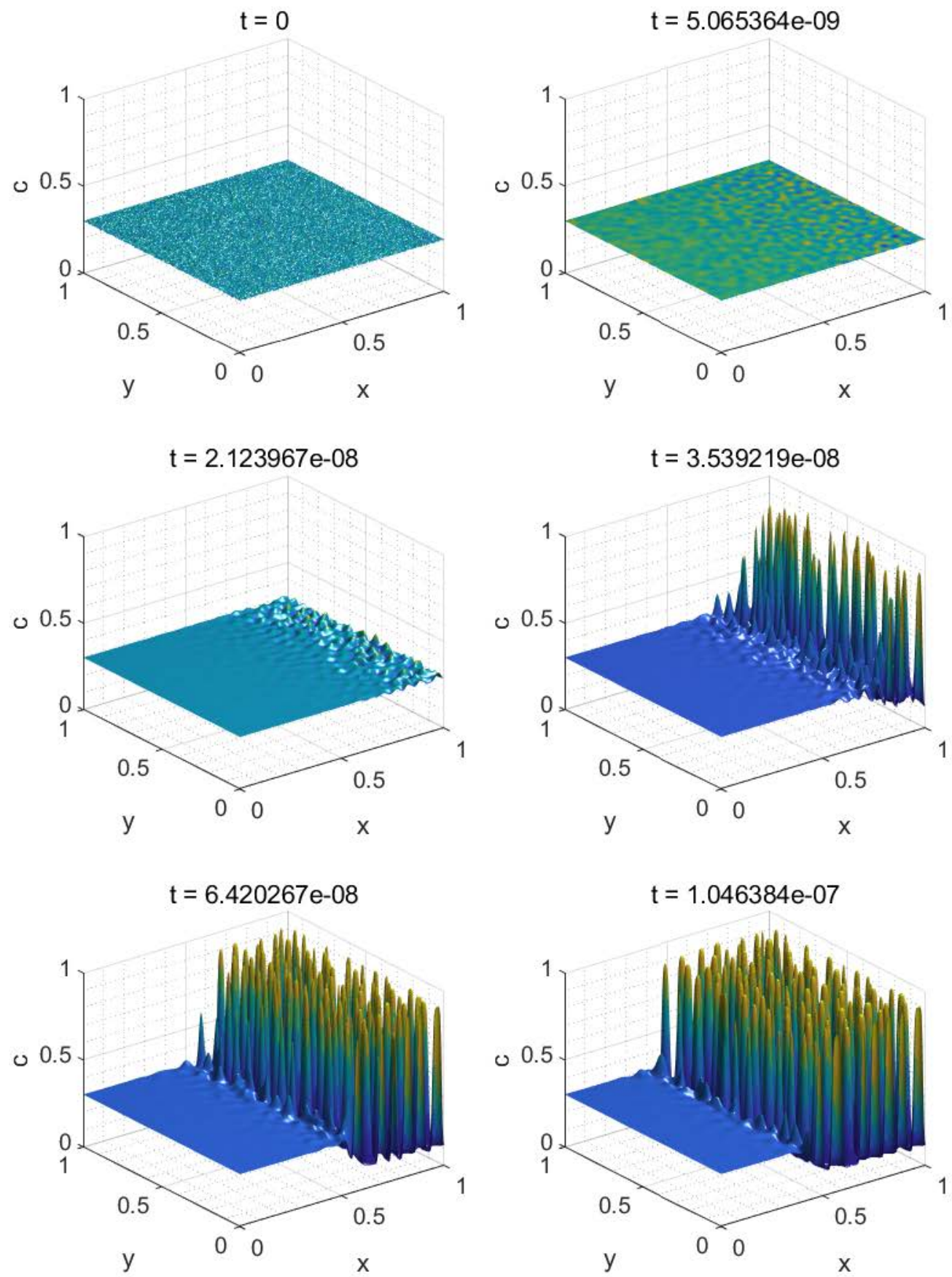

Figure 5.2-1 Concentration profile of the solution with $D=100000, c_{\text {ave }}=0.3, n_{1}=n_{2}=1$ and temperature varies with $T_{1}=0.34$ with $T_{2}=0.30$ (Case 5.2.1.A) 

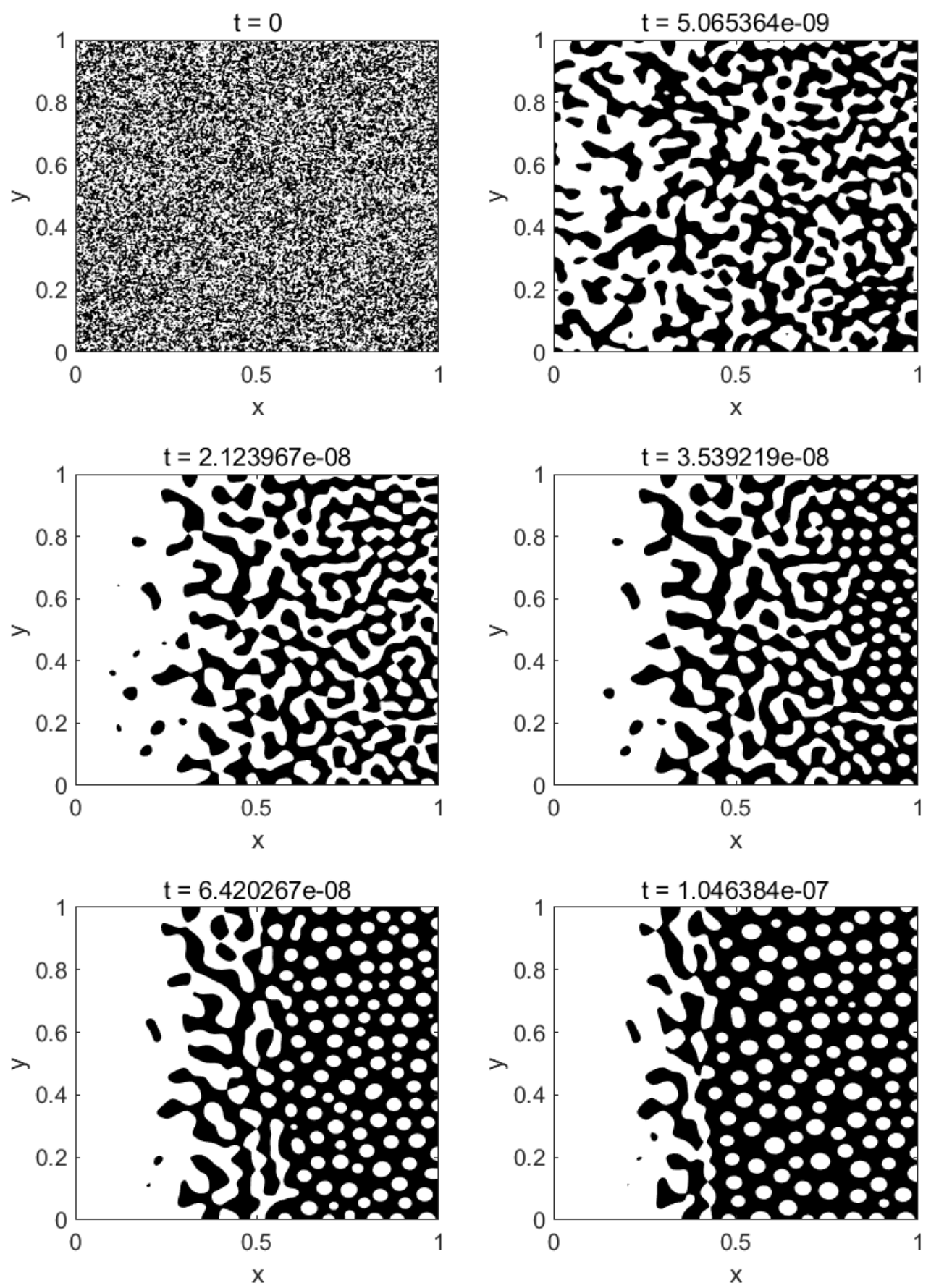

Figure 5.2-2 Two-colours contour plots of the same simulation as in Figure 5.2-1 (Case 5.2.1.A) 

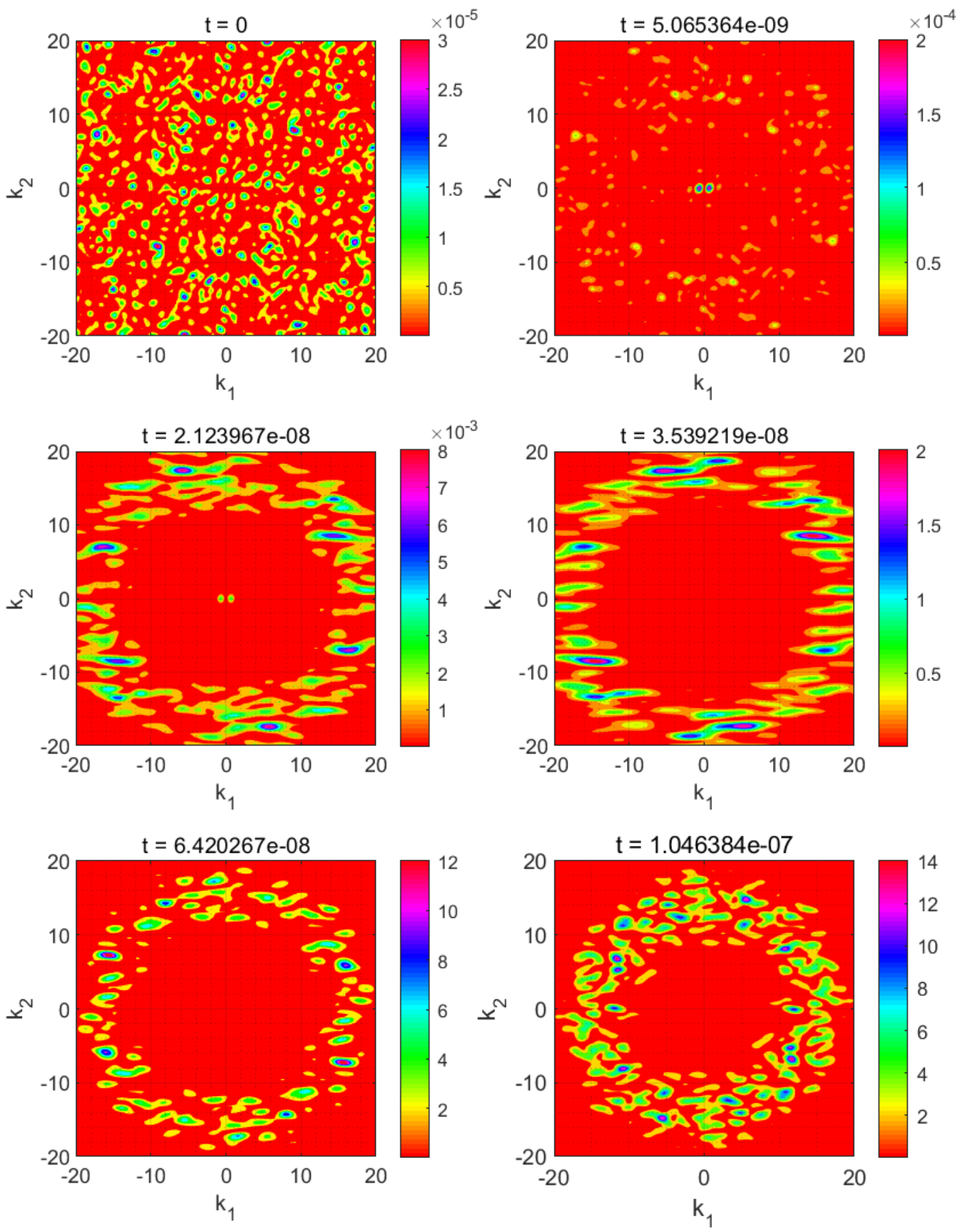

Figure 5.2-3 Structure factors of configurations of the same simulation as in Figure 5.2-1 (Case 5.2.1.A) 

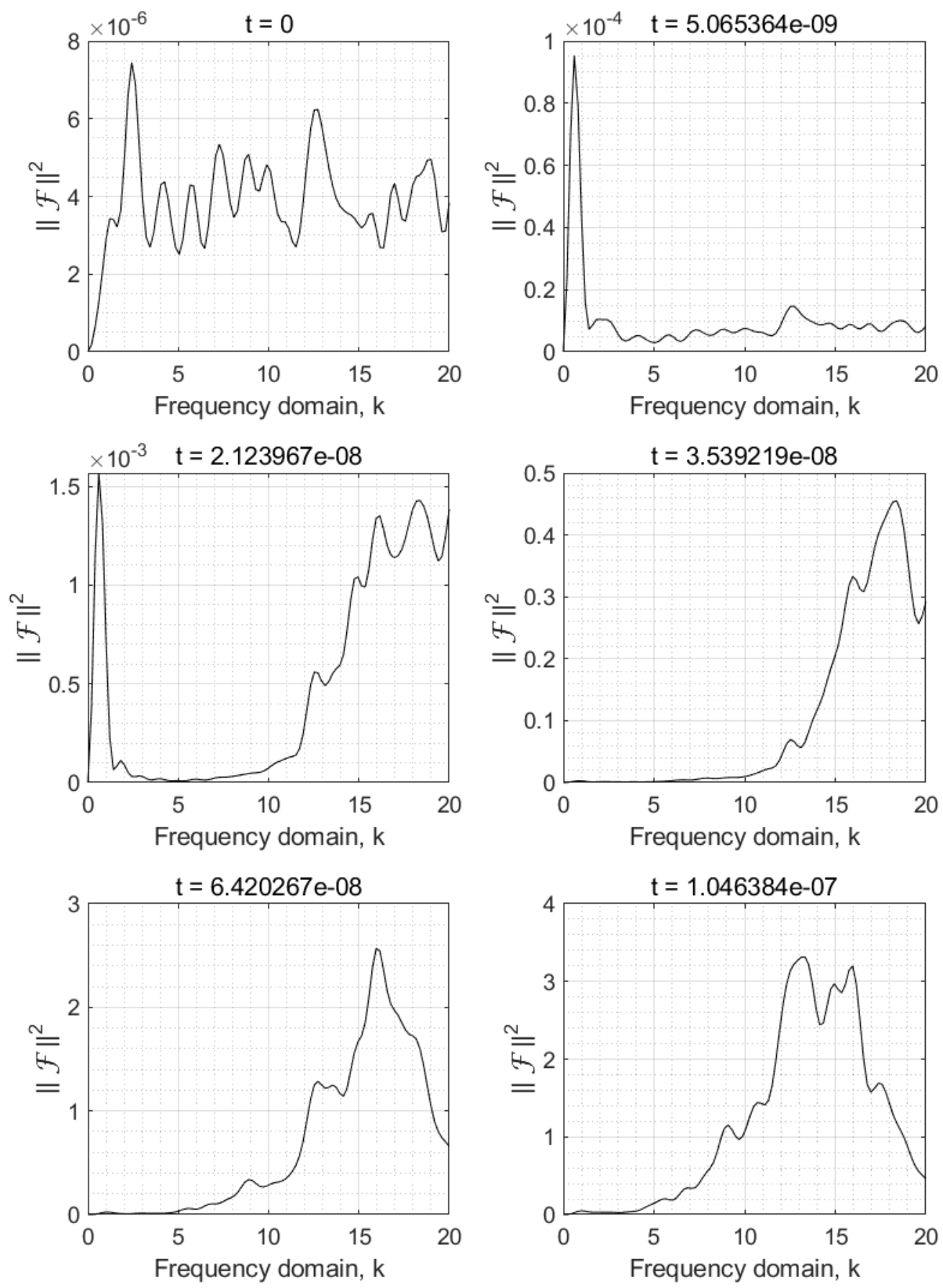

Figure 5.2-4 Circularly-averaged structure factor of the same simulation as in Figure 5.2-1 (Case 5.2.1.A) 

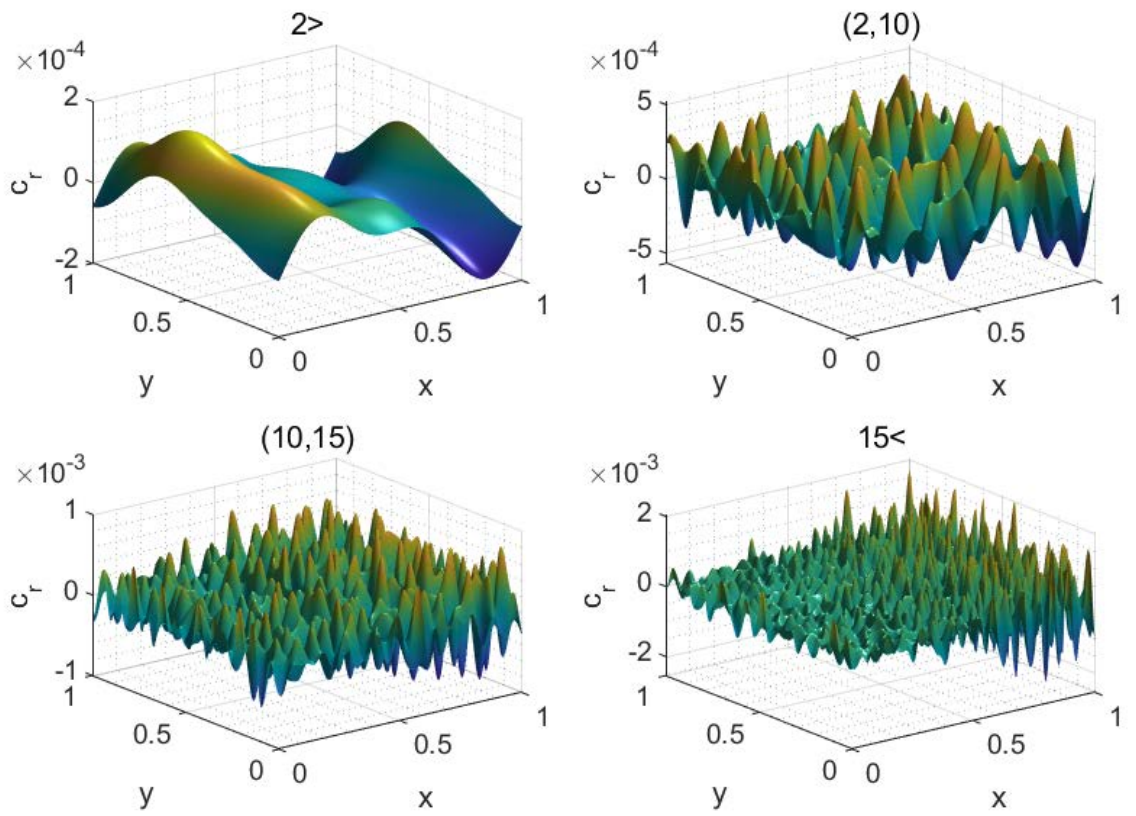

Figure 5.2-5 Band-pass filter is applied to configuration at $t=5.065364 \times 10^{-9}$ of the same simulation as in figure 5.2-1 (Case 5.2.1.A)
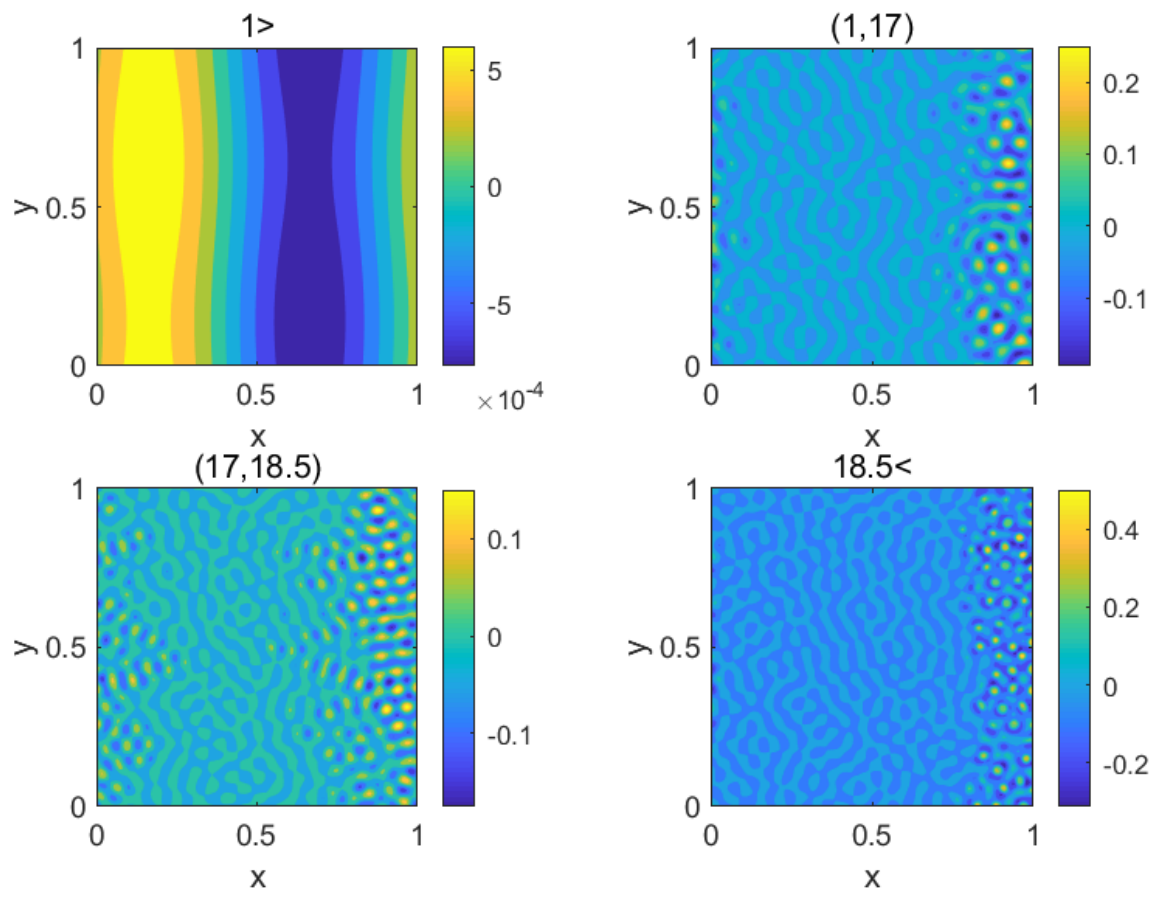

Figure 5.2-6 Band-pass filter is applied to configuration at $t=3.539219 \times 10^{-8}$ of the same simulation as in figure 5.2-1 (Case 5.2.1.A) 


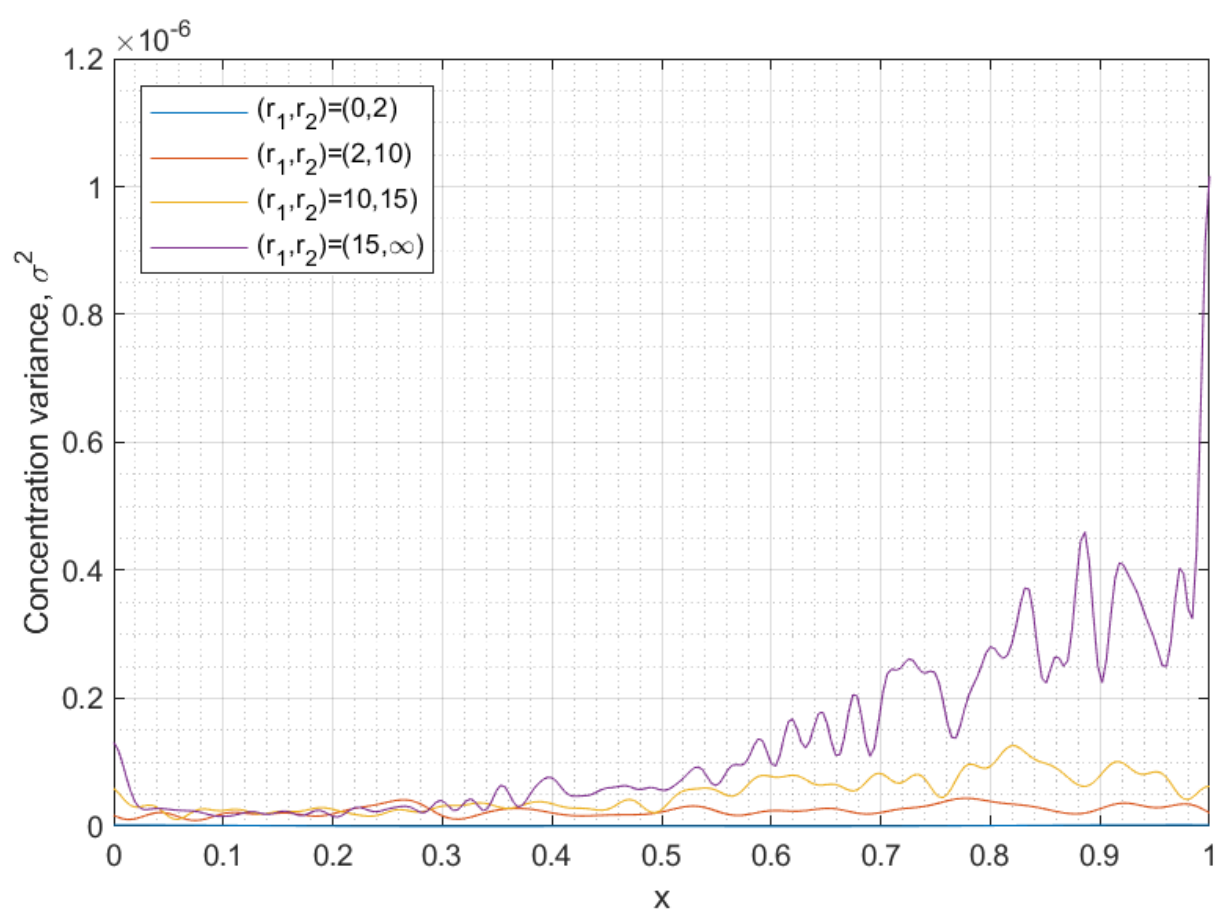

Figure 5.2-7 The variance of concentration of the concentration profiles after band-pass filters were applied at $t=5.065364 \times 10^{-9}$ of the same simulation as in figure 5.2-1 (Case 5.2.1.A)

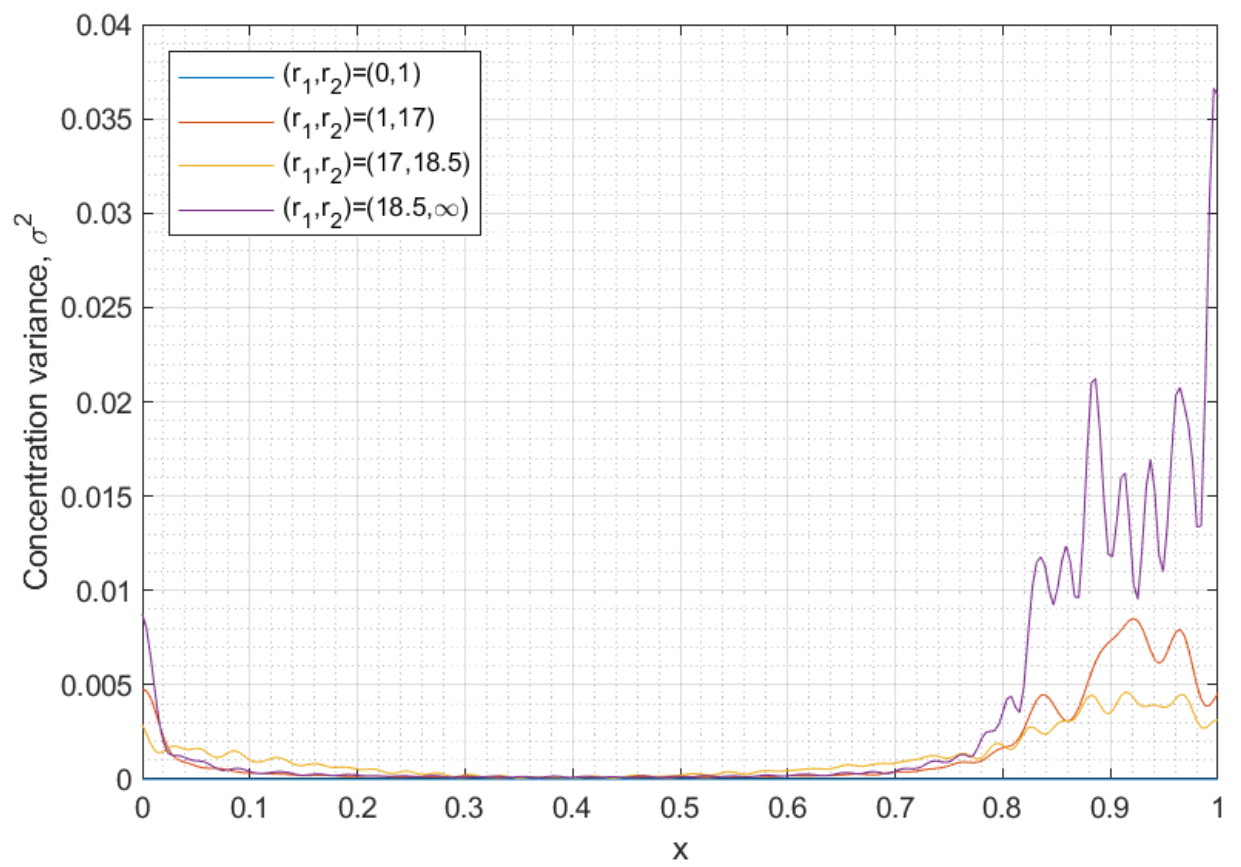

Figure 5.2-8 The variance of concentration of the concentration profiles after band-pass filters were applied at $t=3.539219 \times 10^{-8}$ of the same simulation as in figure 5.2-1 (Case 5.2.1.A) 


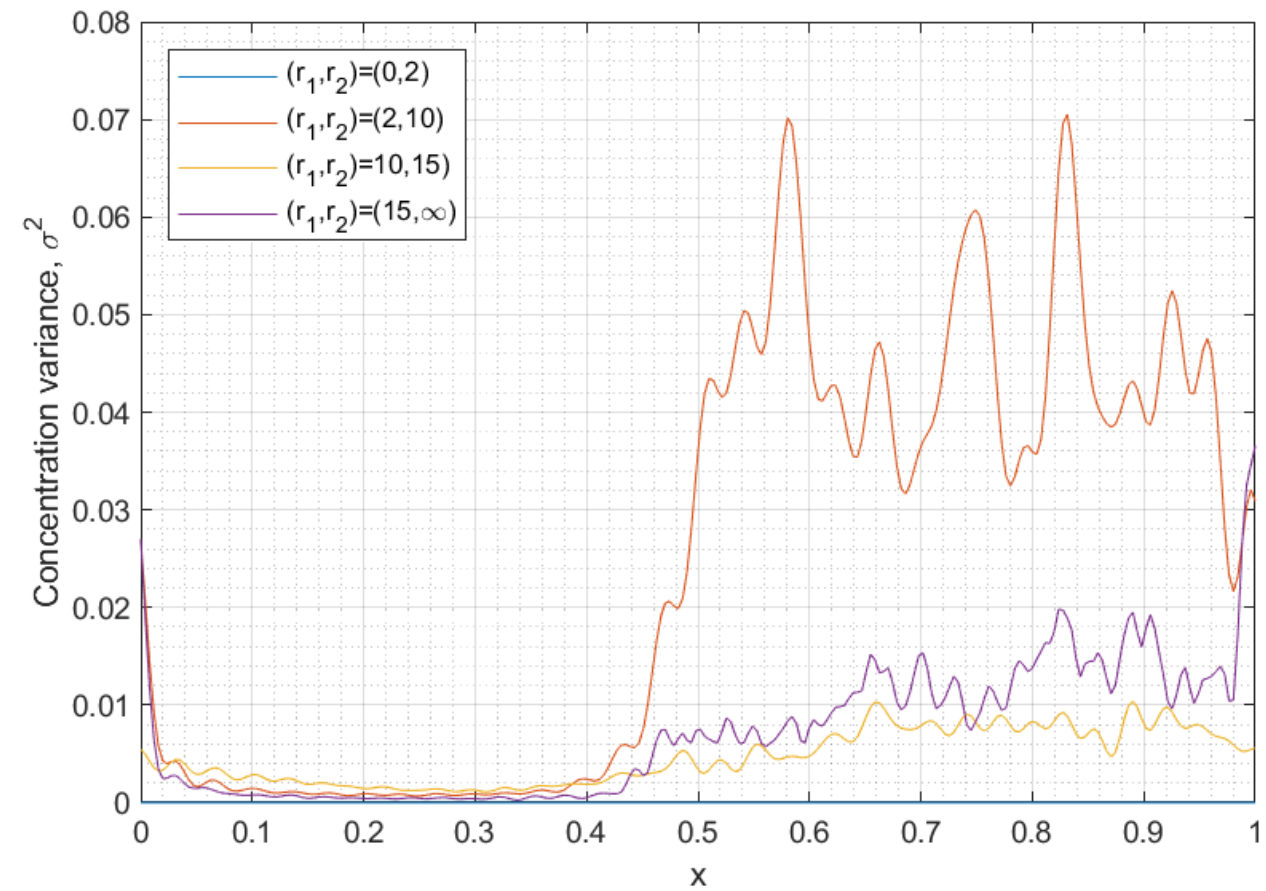

Figure 5.2-9 The variance of concentration of the concentration profiles after band-pass filters were applied at $t=1.046384 \times 10^{-7}$ of the same simulation as in figure 5.2-1 (Case 5.2.1.A)

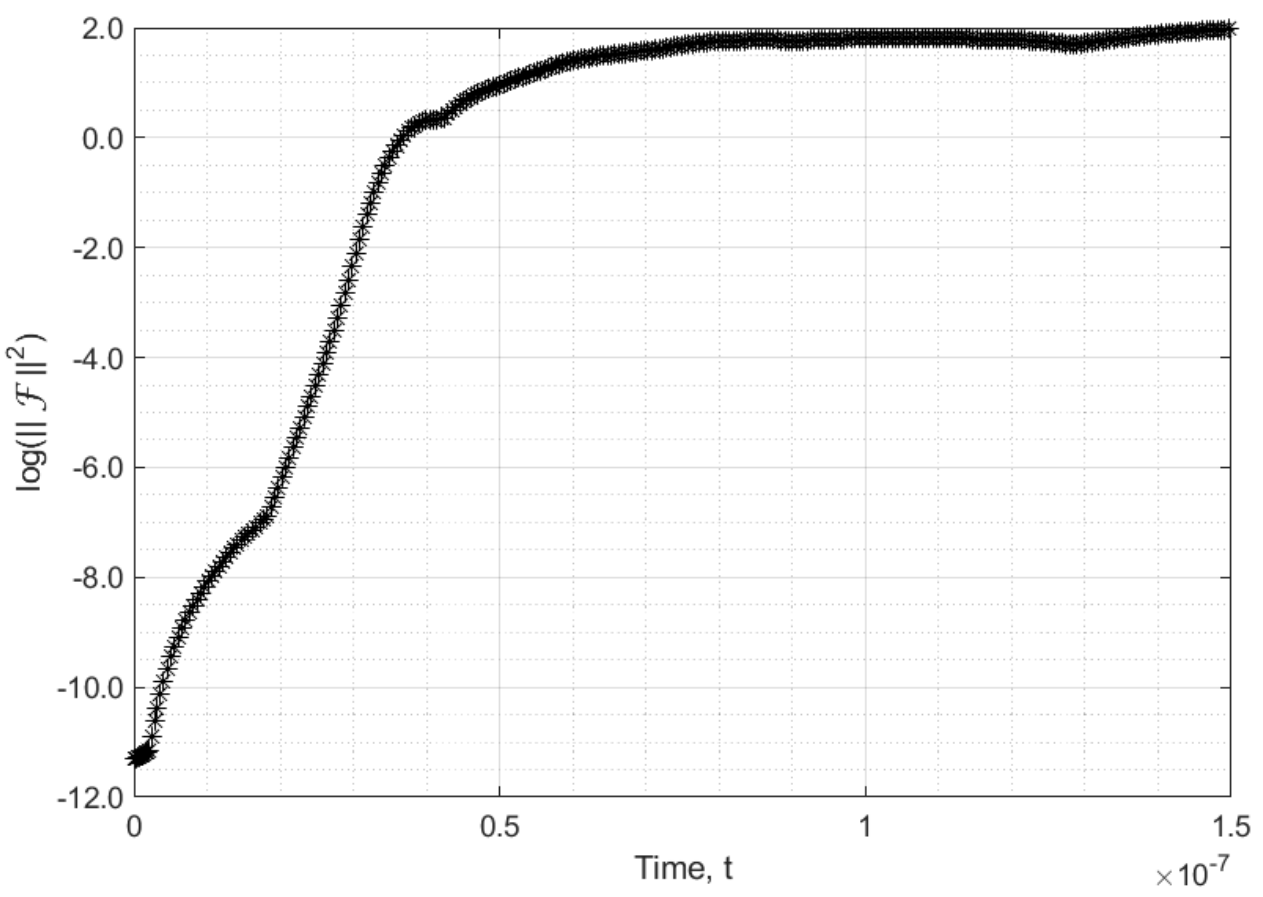

Figure 5.2-10 Log plot of maximum amplitude of the same simulation as in Figure 5.2-1 (Case 5.2.1.A) 


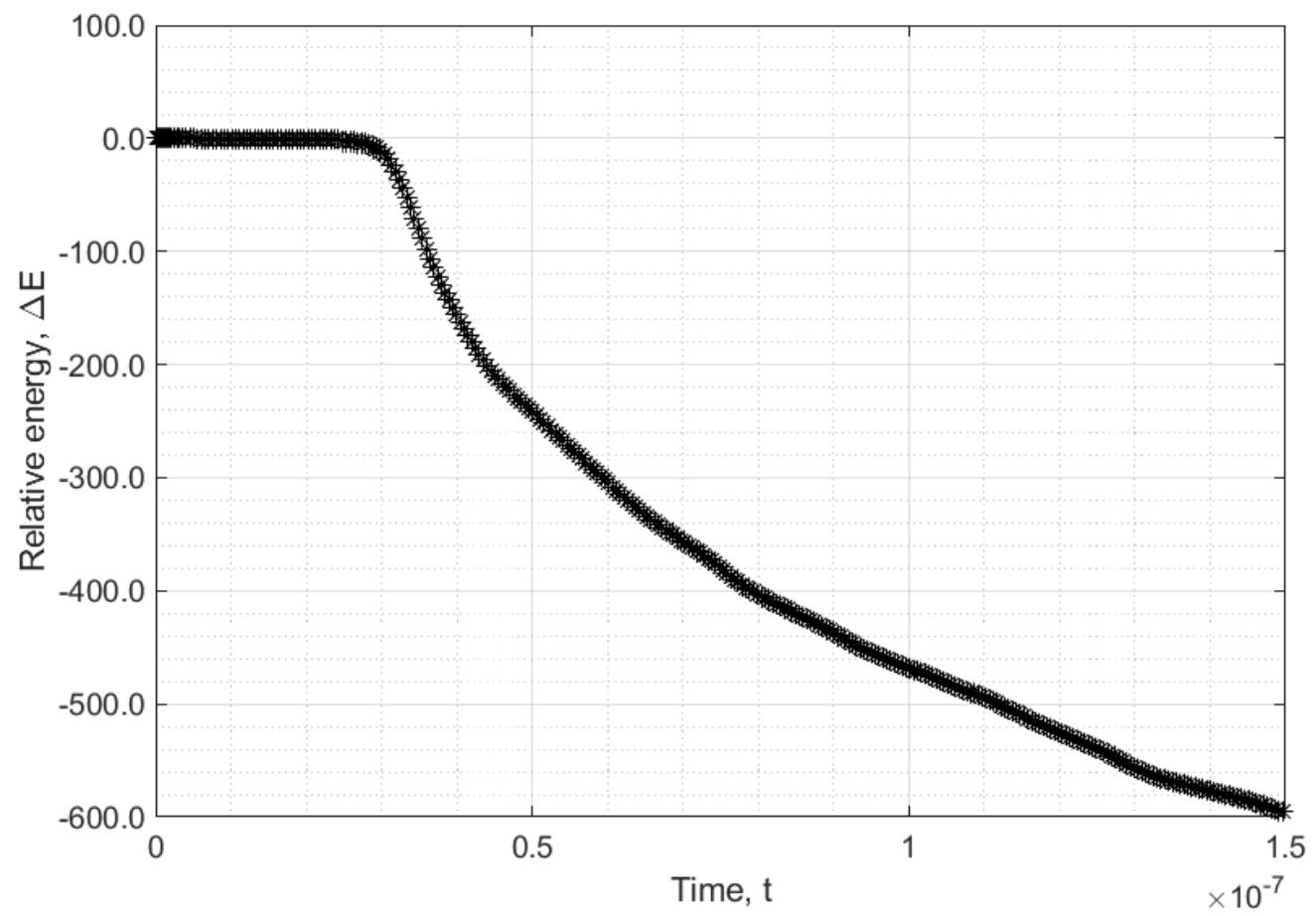

Figure 5.2-11 Relative energy of the same simulation as in Figure 5.2-1 (Case 5.2.1.A)

\subsubsection{Polymerization gradient}

For this research, polymerization gradient was generated. The analysis is very much similar to those with temperature gradient in that it can generate varying structures across the domain. The observation is omitted, but it is mentioned here as reader may find it useful for next sub-section.

\subsubsection{Biaxial gradient}

So far, anisotropic quenching by creating temperature or polymerization gradient has been carried out. The result is a structures that significantly vary across the domain. By creating gradient in one direction, morphology varies only in such direction uniaxially. If different parameters are independent from one another, then anisotropic quenching with multi different parameters may be feasible. Again, the idea of polymerization gradient is rather theoretical because in experimental settings, other effects such as viscosity and elasticity become significant, which are not accounted for in present model. Nonetheless, it is interesting to vary 
multiple parameters at the same time. In order to distinguish one effect from the other, it is ideal to have the components vary in the directions that are diagononal to one another. In Hirose et al.'s research [23], they have suggested that the biaxially varying morphology has been obtained using two different irradiation methods in two directions. They used polymer blend of Poly(ethyl acrylate)/poly(methyl methacrylate) (PEA/PMMA). In their publication, two different irradiation methods are used to generate a gradient of quench depth. One is simply by irradiating strong light intensity in Z-direction. The other is to use computer-assisted irradiation (CAI) method to create a gradient of light intensity. Though yet formally presented, they claimed that they have obtained biaxially graded morphology inside xy plane as well as z direction.

For this thesis, because the model for the gradients in temperature and polymerization has been established, simulations with gradients in these two parameters can be carried out. Figure 5.2-12 shows the overall phase separation process for Case 5.2.3.A where $D=60000$, $c_{\text {ave }}=$ 0.7 and $n_{1}=1$. Here, the gradients are established such that

$$
\begin{gathered}
\left.T\right|_{x=0}=0.34 \\
\left.T\right|_{x=1}=0.3 \\
\left.\nabla T\right|_{x=0}=\left.\nabla T\right|_{x=1}=\overrightarrow{0} \\
\left.n_{2}\right|_{y=0}=1 \\
\left.n_{2}\right|_{y=1}=1.3 \\
\left.\nabla n_{2}\right|_{y=0}=\left.\nabla n_{2}\right|_{x=1}=\overrightarrow{0}
\end{gathered}
$$

The temperature and polymerization fields are the function of third degree polynomials that satisfy all of the condition above. These parameters are deliberately selected so that the quench depth at $(x, y)=(0,0)$ is the lowest, those at $(x, y)=(1,0)$ and $(x, y)=(0,1)$ are about the same, and that at $(x, y)=(1,1)$ is the highest. This way, one may obtain a structure in which phase separation is expected to occur earliest near deepest quench corner, $(x, y)=$ $(1,1)$, then it propagates towards the shallowest quench corner at $(x, y)=(0,0)$. The description of the overall process are similar to what has been described for uniaxially graded morphology created by temperature and polymerization gradient. That is, there is a rise in low 
frequency structures that forms into the shape of the gradient created by the difference in potential energy across the domain. There is also a spinodal decomposition in which the phases separate by local fluctuation in concentration. To better illustrate this, Figure 5.2-16 shows the structures with band-pass filters applied at $t=3.539219 \times 10^{-8}$. Indeed, the structure with $f<1$ has formed into 2 -d sinusoidal like shape by the gradients. The analysis of this observations is similar as above, except this time, graded morphology in two perpendicular directions is obtained. 

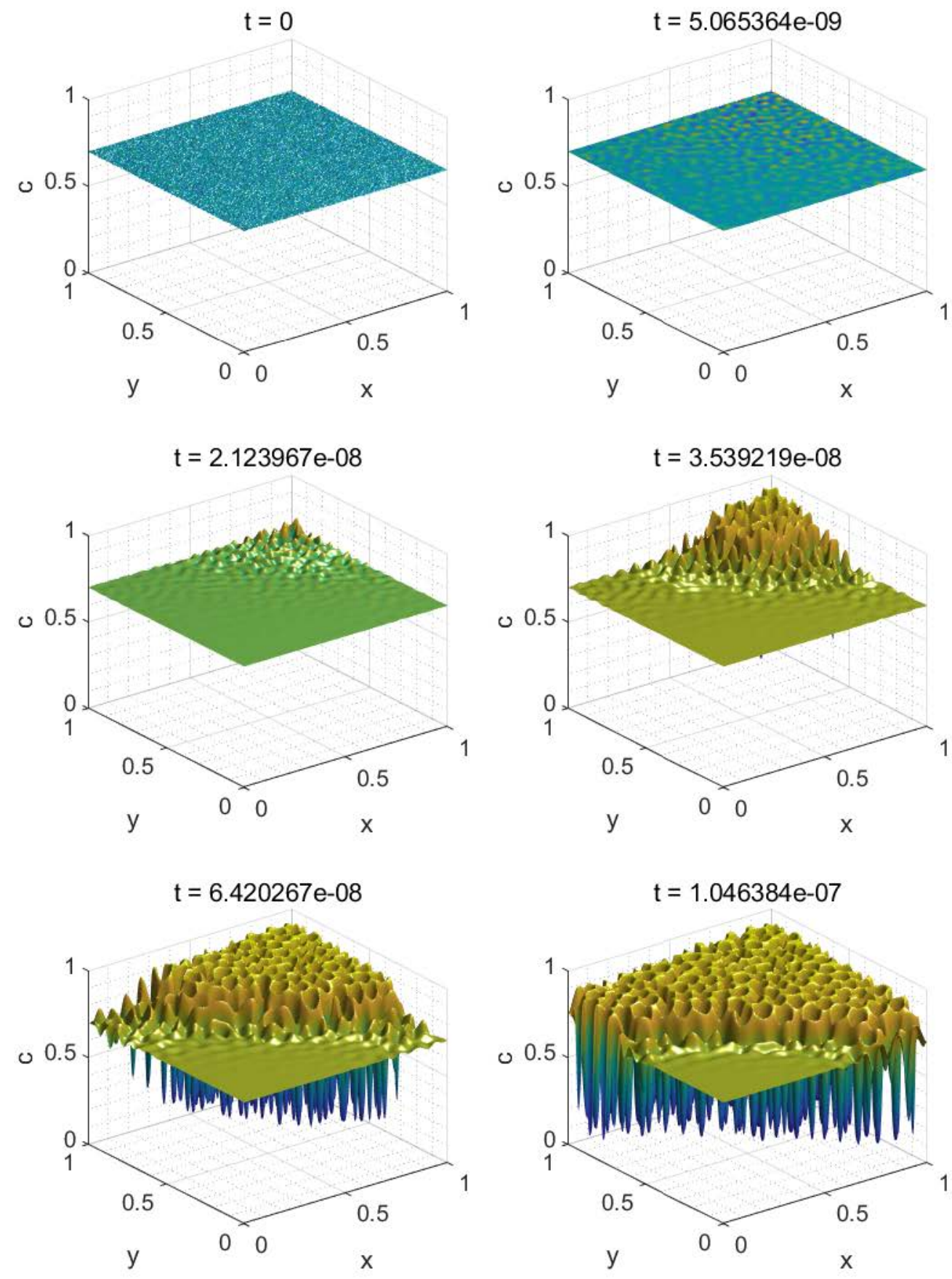

Figure 5.2-12 Concentration profile of the simulation with $D=60000, c_{\text {ave }}=0.7$ and $n_{2}=1$. The temperature varies in along $x$-direction with $T=0.34$ at $x=0$ and $T=0.3$ at $x=1$. Polymerization varies in $y$-direction with $n_{2}=1$ at $y=0$ and $n_{2}=1.3$ at $y=1$ (Case 5.2.3.A) 

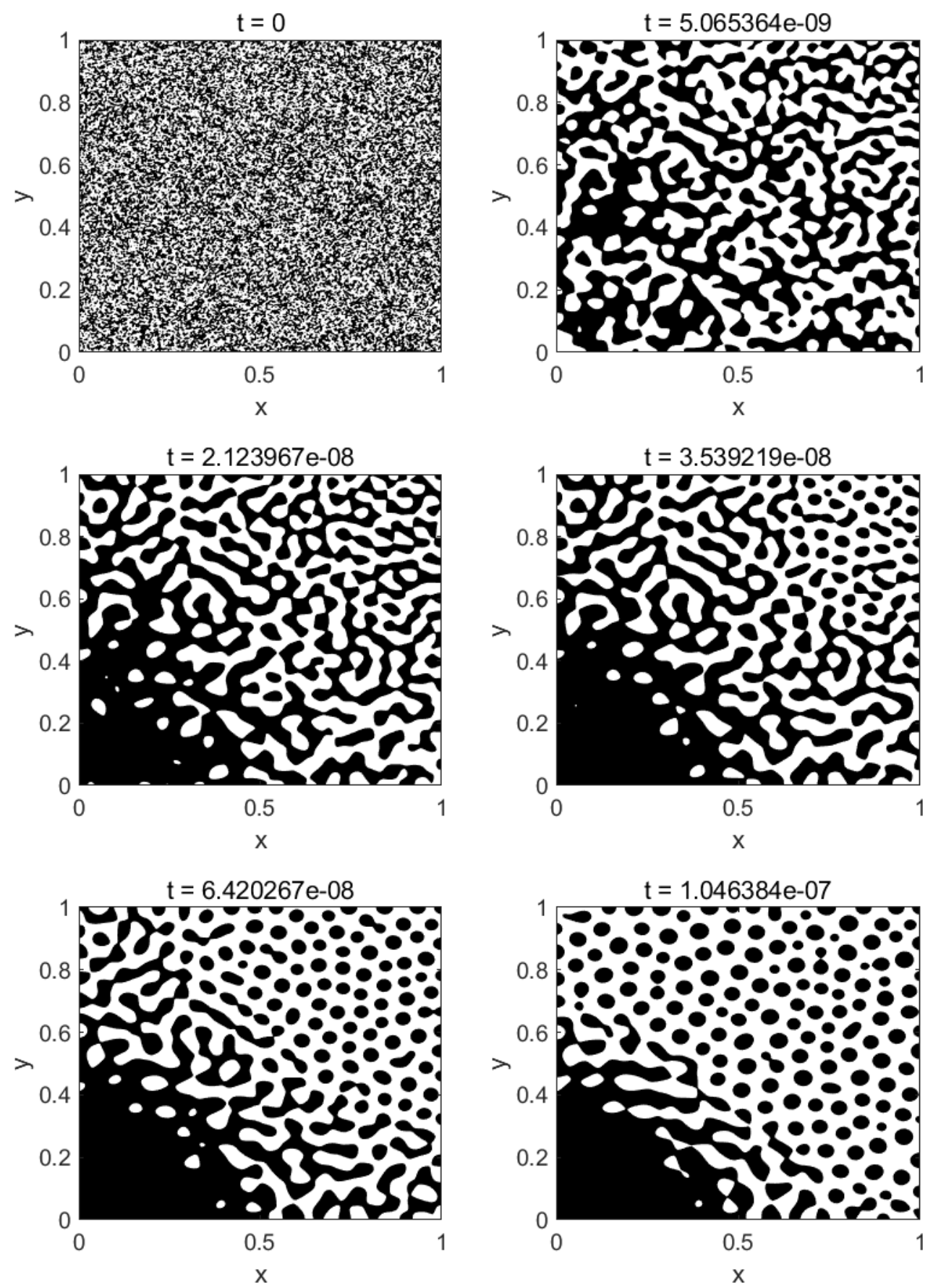

Figure 5.2-13 Two-colors contour plot of the same simulation as in Figure 5.3-9 (Case 5.2.3.A) 

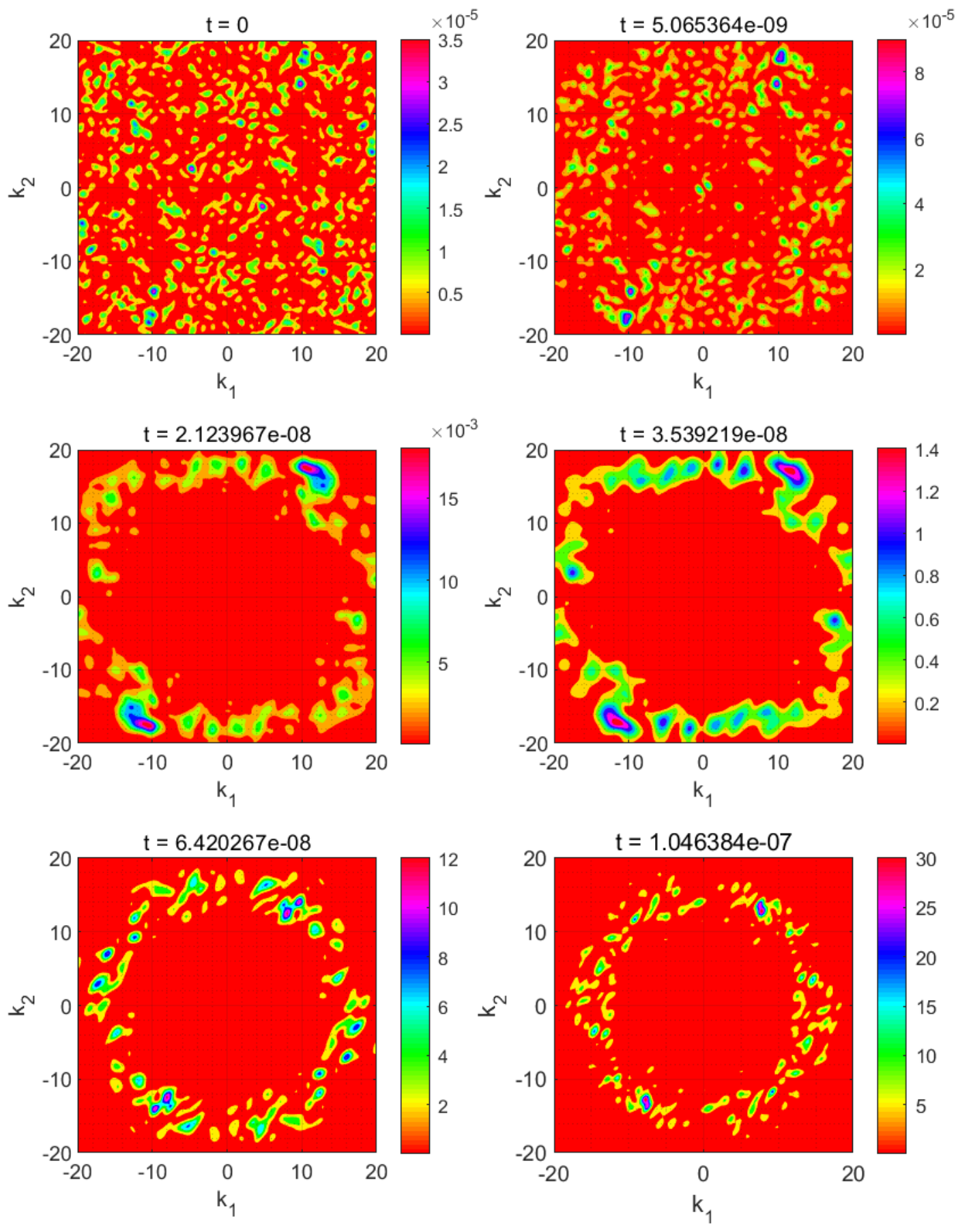

Figure 5.2-14 Structure factor of the same simulation as in Figure 5.3-9 (Case 5.2.3.A) 

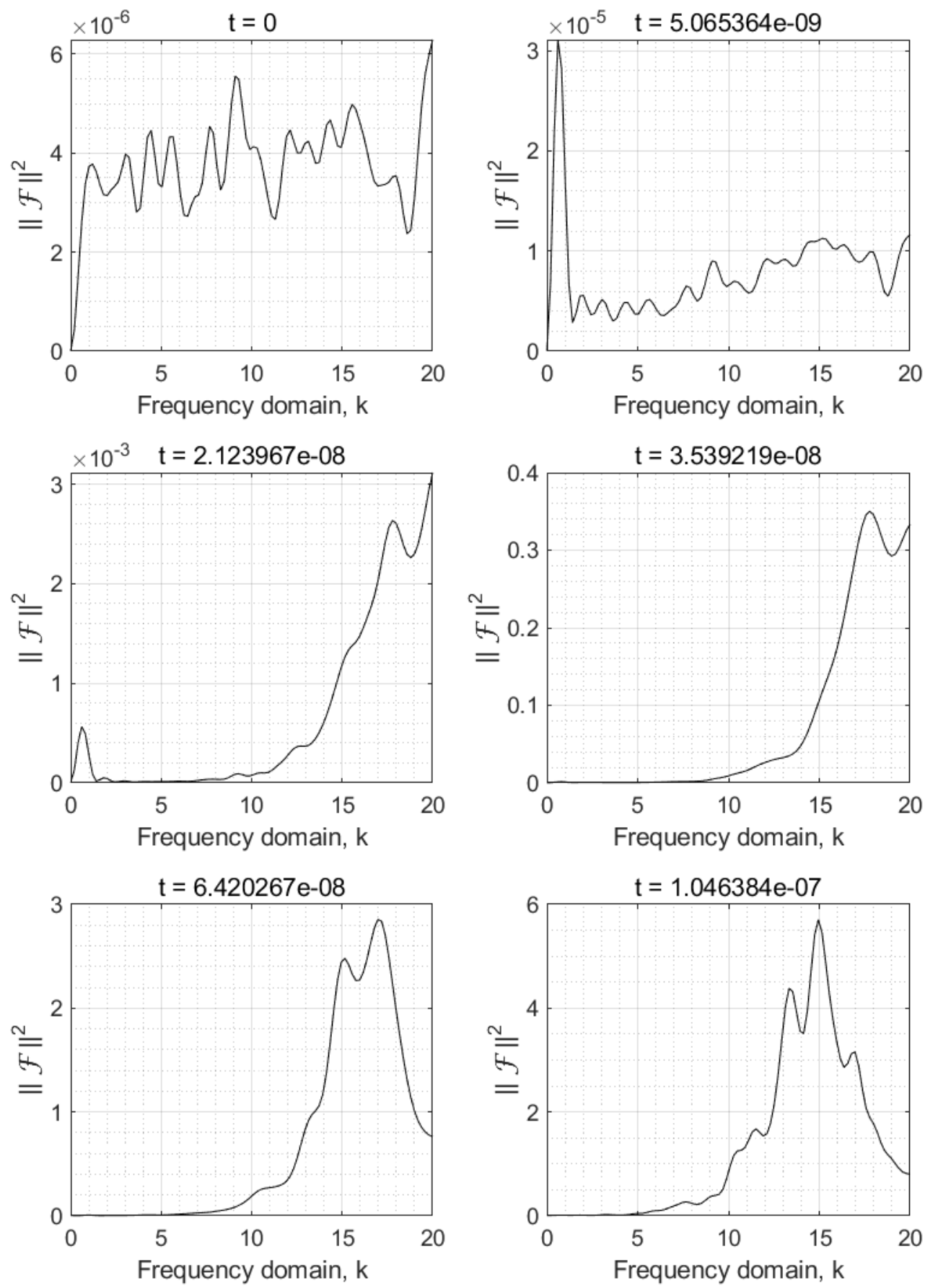

Figure 5.2-15 Circularly-averaged structure factor of the same simulation as in Figure 5.3-9 (Case 5.2.3.A) 

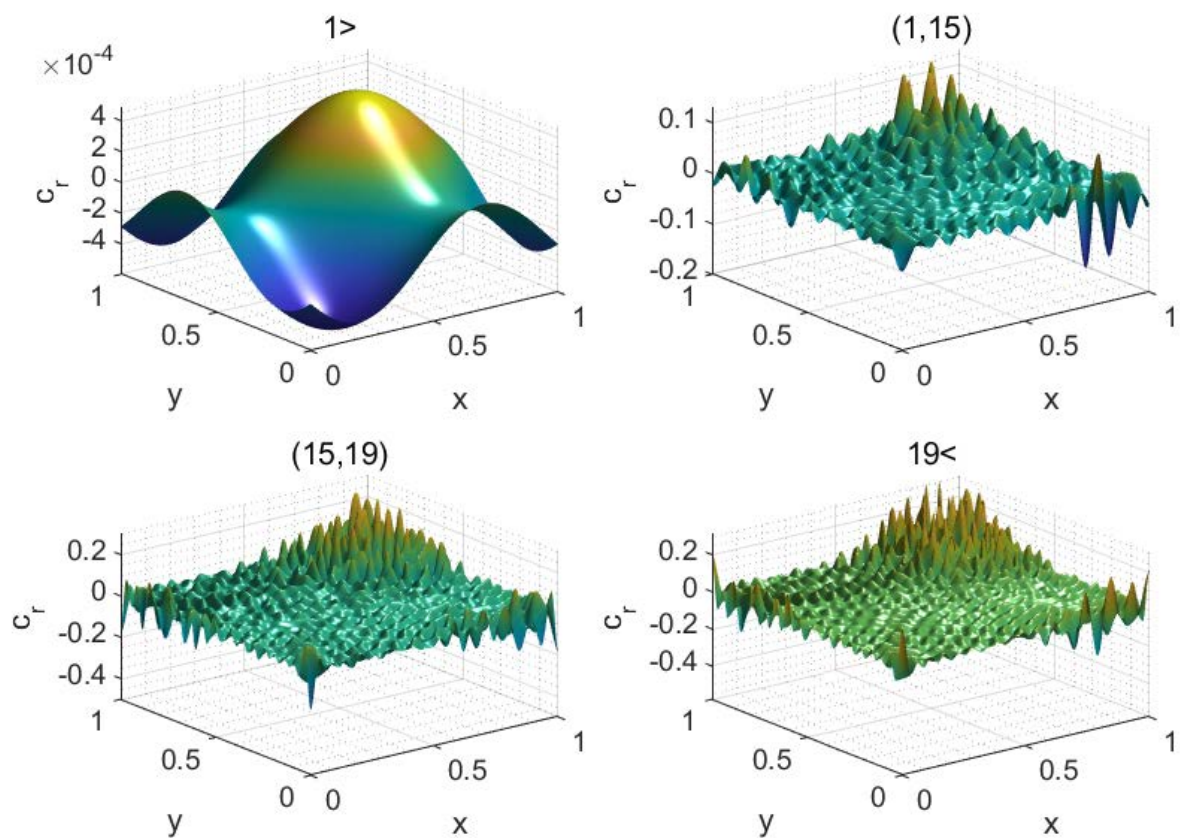

Figure 5.2-16 Band-pass filters of configuration at $t=3.734029 \times 10^{-7}$ (Case 5.2.3.A)

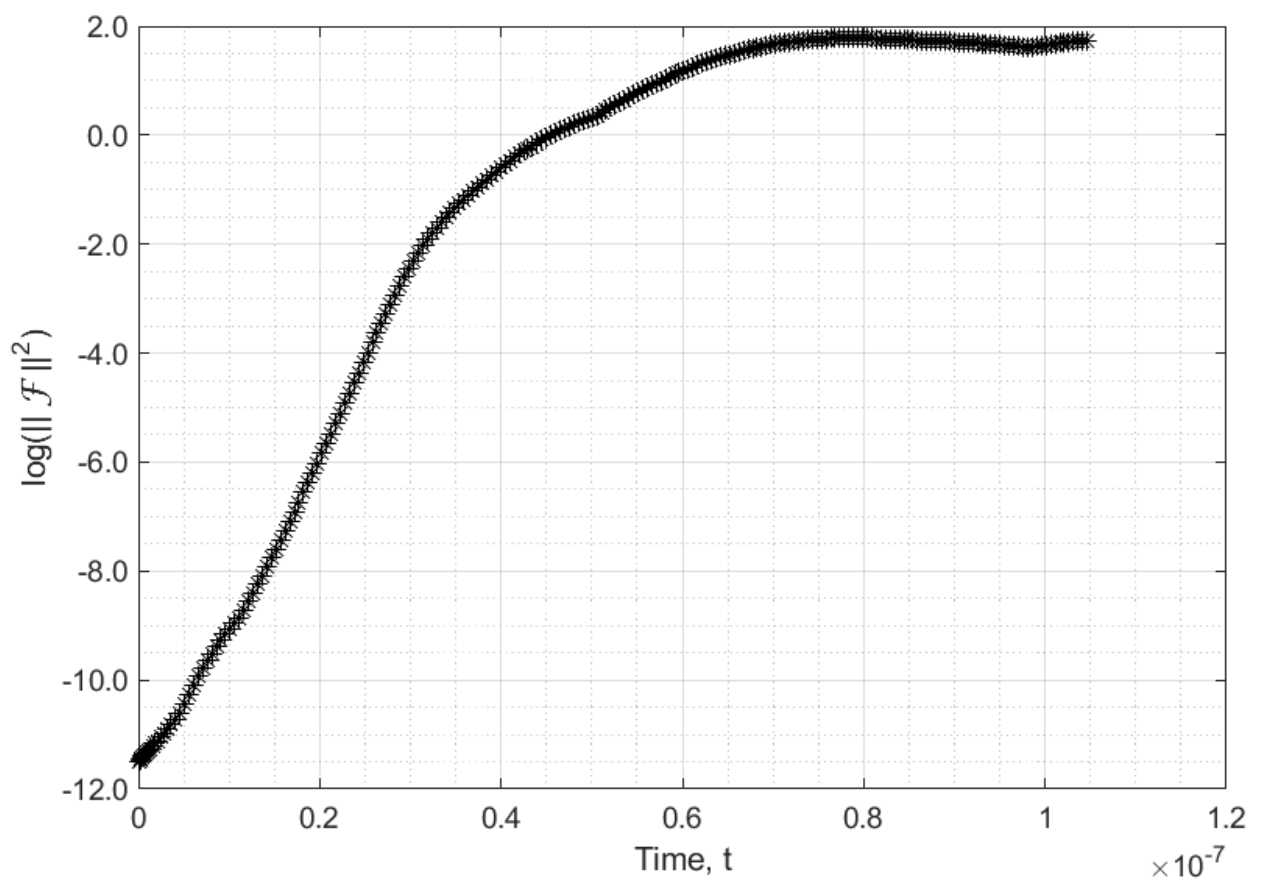

Figure 5.2-17 Maximum amplitude of structure factor of the same simulation as in Figure 5.3-9 (Case 5.2.3.A) 


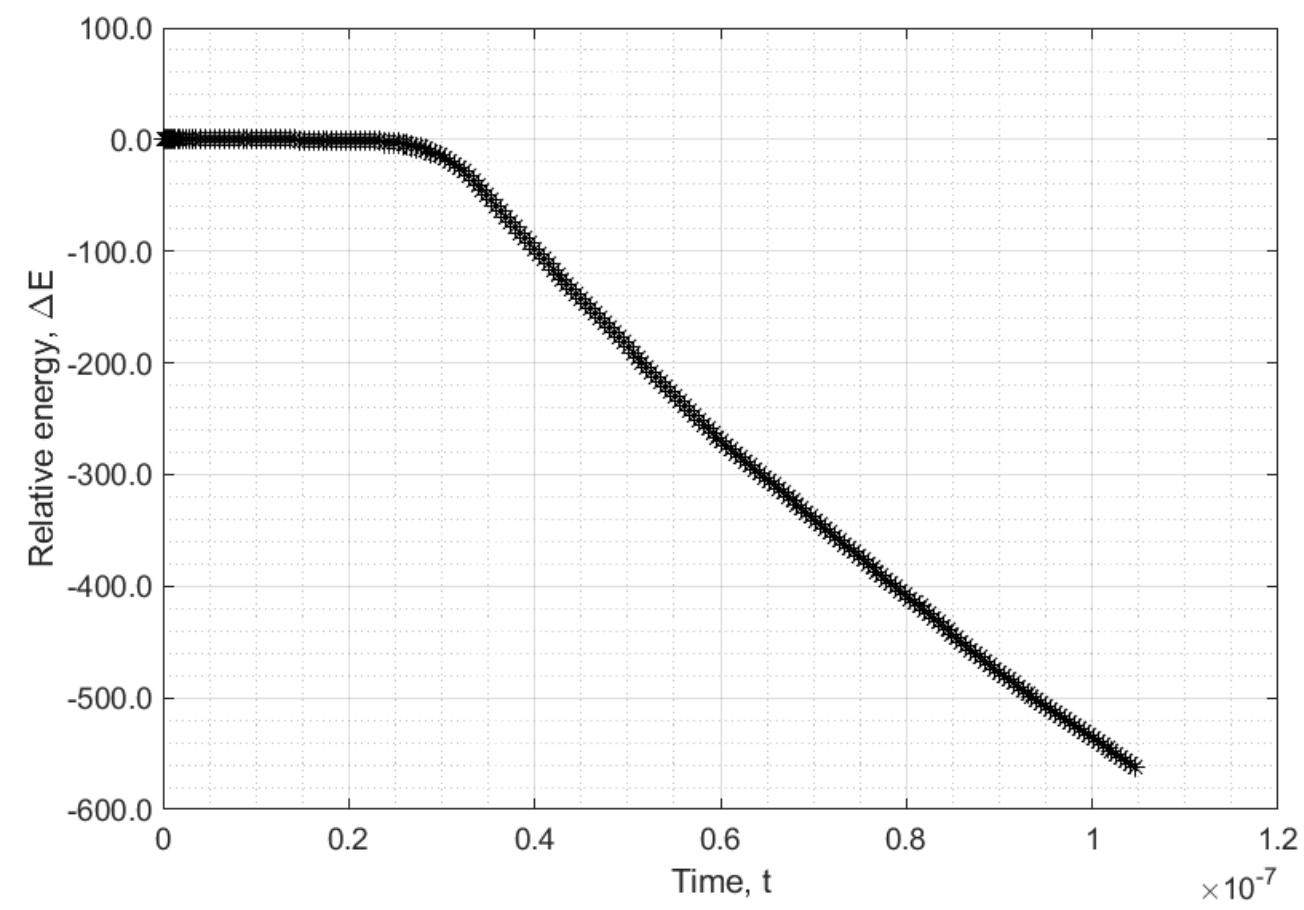

Figure 5.2-18 Relative energy over time of the same simulation as in Figure 5.3-9 (Case 5.2.3.A)

\subsection{Wetting}

So far, all simulations assumed boundary condition at the first derivative of concentration as:

$$
\left.\nabla c\right|_{\partial \Omega} \cdot \hat{n}=0
$$

Where $\hat{n}$ is a unit vector pointing outwards from the surface. This is not always true in nature, as the polymers may sometimes interact with the surfaces in contact with intermolecular forces. Such phenomenon is called the wetting in the polymer and it has been described. In two component polymer blends, such phenomenon is also possible. Schmidt and Binder [70] have suggested the numerical model to study it. Puri and Binder [34] has studied spinodal decomposition under the effect of wetting numerically. Often, it can be simplified as the linear function of concentration, i.e.

$$
\left.\nabla c\right|_{\partial \Omega} \cdot \hat{n}=g c+h
$$


For some constants $g$ and $h \in \mathbb{R}$. Physical meaning of each can be interpreted directly from equation (5.3.2): $h$ is the degree to which component 1 is attracted to independent of concentration, while $g$ manifests how the surface attraction or repulsion is dependent on concentration. In spinodal decomposition, generally, there is a competition between two processes: one is the usual spinodal decomposition, which yields a random structure. The other is the development in fluctuation due to the effect of wetting. According to equation (5.3.2), the wetting "bends" the layer of concentration, ultimately leading to some fluctuation immediately adjacent to the boundary. The energy is a summation of local energy density and the squared absolute of concentration gradient, the interfacial energy between the polymer blend and the boundary is no longer zero. So, reasoning for some phenomena observed so far, such as the tendency of higher frequency structures to stay at the boundaries of the domain because of zero interfacial energy barrier, is no longer valid. By taking advantage of force balance between adhesive and cohesive forces, one may strategically use surface to direct the course of polymer separation. Furthermore, it has been shown that different wetting patterns at different surfaces may generate variety of peculiar morphologies.

For this section, the same method is used to calculate total relative energy, which does not account for initial wetting, so one may expect to see a rise in relative energy. This time, instead of 2-d, 3-d model is used as wetting is normally applied with 2-d surface in real world. In order to reduce computational burden, serendipity function is used instead, i.e. the mixed term is no longer present at every node. By eliminating mixed term, one can also avoid complication arisen from non-constant boundary condition as explained section 3.9.2. In previous researches by others, it has been shown that, for certain parameters, the layer of fluctuation created by wetting layer propagates slower over time. Nonetheless, because they keep proceeding, it is interesting to note any effect created by it over long time. For the first time, growth and nucleation in metastable region without external noise, but with wetting effect, is observed numerically and described. Because spinodal decomposition is minimal in metastable region, the patterns created are quite well-defined without much external influence, and minimal asymmetricity created from the initial fluctuation of concentration. 
Figure 5.3-1 and Figure 5.3-2 show the overall process of metastable phase separation with $D=5000, c_{\text {ave }}=0.3, T=0.35, n_{1}=n_{2}=1$ (Case 5.3.A). The wetting parameters are $g=0$ and $h=-1.0$ at all boundary surfaces. This may be referred to as long-range surface-directed phase separation, as the wetting is present at all boundaries. For negative $h$ value, major component, or component 2 , is attracted towards the wall. Immediately after the simulation starts, the interfacial layer develops in cubic like shape within the domain at $t=2.864835 \times$ $10^{-5}$. Here, Figure 5.3-3 shows that there is anisotropy in $x, y$, and $z$ directions, as highamplitudes are shown along the axis of $k_{1}=0, k_{2}=0$, and $k_{3}=0$. These correspond to cubic-like structures that formed within the domain. In closer look at Figure 5.3-4, which shows circularly averaged structure factor, there are three most dominant peaks at about $f=1,2$ and 3.1. They are in descending orders. Upon applying band-pass filters at the configuration of $t=2.864835 \times 10^{-5}$ in Figure 5.3-5, there are several droplets attempting to evolve at different frequencies. The structure at $f=1$ corresponds to one large droplet, while that at $f=2$ has about equally shaped droplets that number in 2 in each direction, while that $f=3$ corresponds to structure that has 3 droplets in each direction. In each structure, all droplets seem to have about equal size, and fit tightly snugly into the domain. They are very welldefined due to the nature of symmetricity experienced as a result of wetting layer of all directions. Because they are so well-structured, spinodal decomposition is expected to be minimal because otherwise, one would expect to see more randomly distributed droplets. As concentration fluctuation is brute-forced with the effect of wetting, the solution attempts to adopt to morphology that has less energy, which is in spherical shape, or droplets, therefore the shapes. Over time, the structure at $f=2$ dominates, as seen at $t=7.879265 \times 10^{-5}$. Plausible explanation is the symmetricity: Because the effect of the wetting is felt most strongly at 8 corners of the domain, it ultimately makes $f=2$ structure most favorable as this symmetrically yields droplets at each corner. Indeed, in concentration profile in Figure 5.3-2, there are eight droplets that has just merged. Note that by this time, the process of NG has already occurred, as the maximum and minimum values of concentration has reached close to respective equilibriums $\left(e_{1}=0.1839\right.$ and $\left.e_{2}=0.8161\right)$. To analyze the structures individually, Figure 5.3-6 shows the log plot of amplitude at different frequencies until $t=7.879265 \times$ $10^{-5}$. Here, structure with $f=2$ dominates over this entire time range. There is a slight 
increase in the rate for $f=2$ from about $t=5.2 \times 10^{-5}$. This is when the droplet at each corner emerges. The rest of the simulation is assymetrical merging of all droplets. They take relatively longer time. Eventually, one large macro-sphere forms at $t=5.524906 \times 10^{-3}$ and it attaches to the wall. The asymmetricity is due to slight random initial fluctuation. Thus, only until very later time, the randomness of initial fluctuation becomes significant. Figure 5.3-7 shows the change in relative energy over time. First of all, a slight increase in relative energy is seen as a result of wetting. Several huge drops in energy is observed at about $t=3.0 \times 10^{-4}$, $t=1.9 \times 10^{-3}$, and $t=3.5 \times 10^{-3}$, just to mention a few. The first large drop is when the droplets form at each corner, while all others mentioned correspond to merging of the droplets into larger ones. For this reason, the stages between the emergences of new droplets are very slow process. But once it has reached a certain threshold, merging of the droplets happens very quickly.

In this chapter, NG under long-range surface-directed wetting has been shown and described. Because it is thrust into metastable region where the effect of random initial fluctuation in concentration is much less significant, the effect of symmetrical wetting prevails. The result is the formation of the new droplets that are very well sorted with the domain. The diffusion by wetting process is slow, therefore every time new droplets form, they tend to stay for a while. One a certain threshold is reached, the droplet merges very quickly to form larger droplets. 

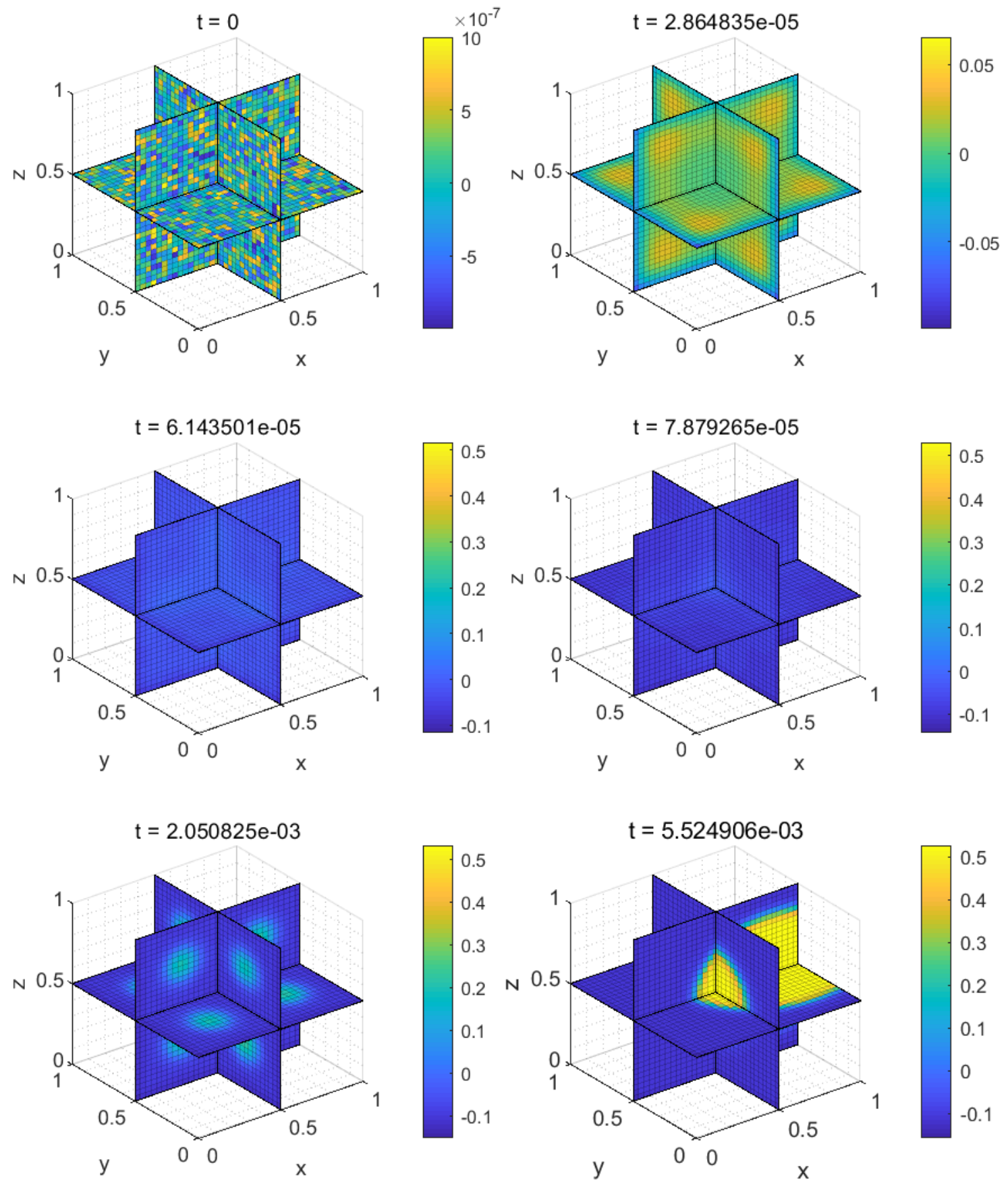

Figure 5.3-1 Slice plots of relative concentration profile for $D=5000, c_{\text {ave }}=0.3, T=$ $0.35, n_{1}=n_{2}=1$. The wetting effect is present at all boundary surfaces with $g=0$ and $h=$ -1.0 . The concentration on $x y, x z$, and $y z$ planes are shown. (Case 5.3.A) 

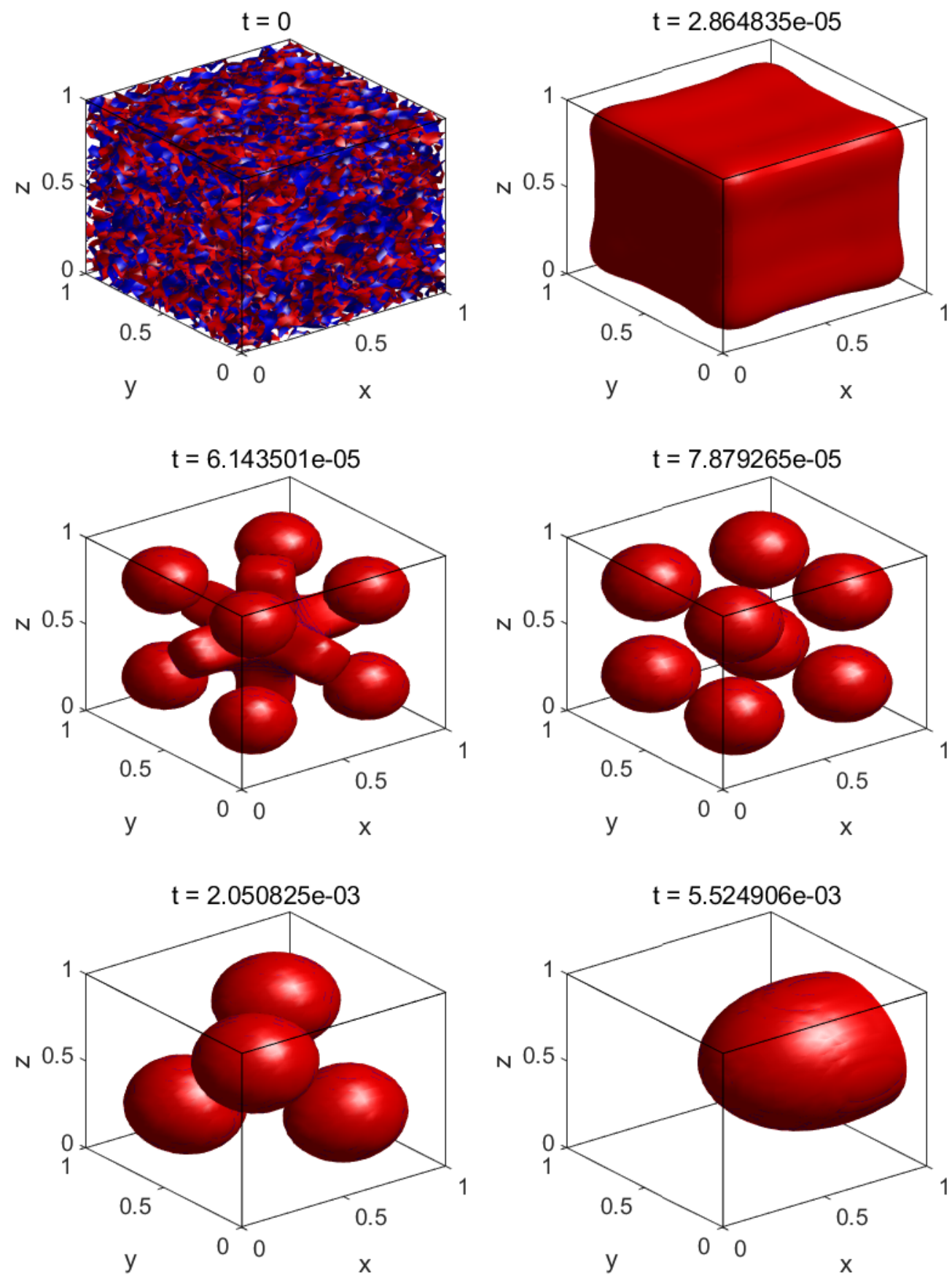

Figure 5.3-2 Isosurface plots of simulation shown in Figure 5.3-1. Blue surface is component-1 rich side, while red surface is component-2 rich side. (Case 5.3.A) 

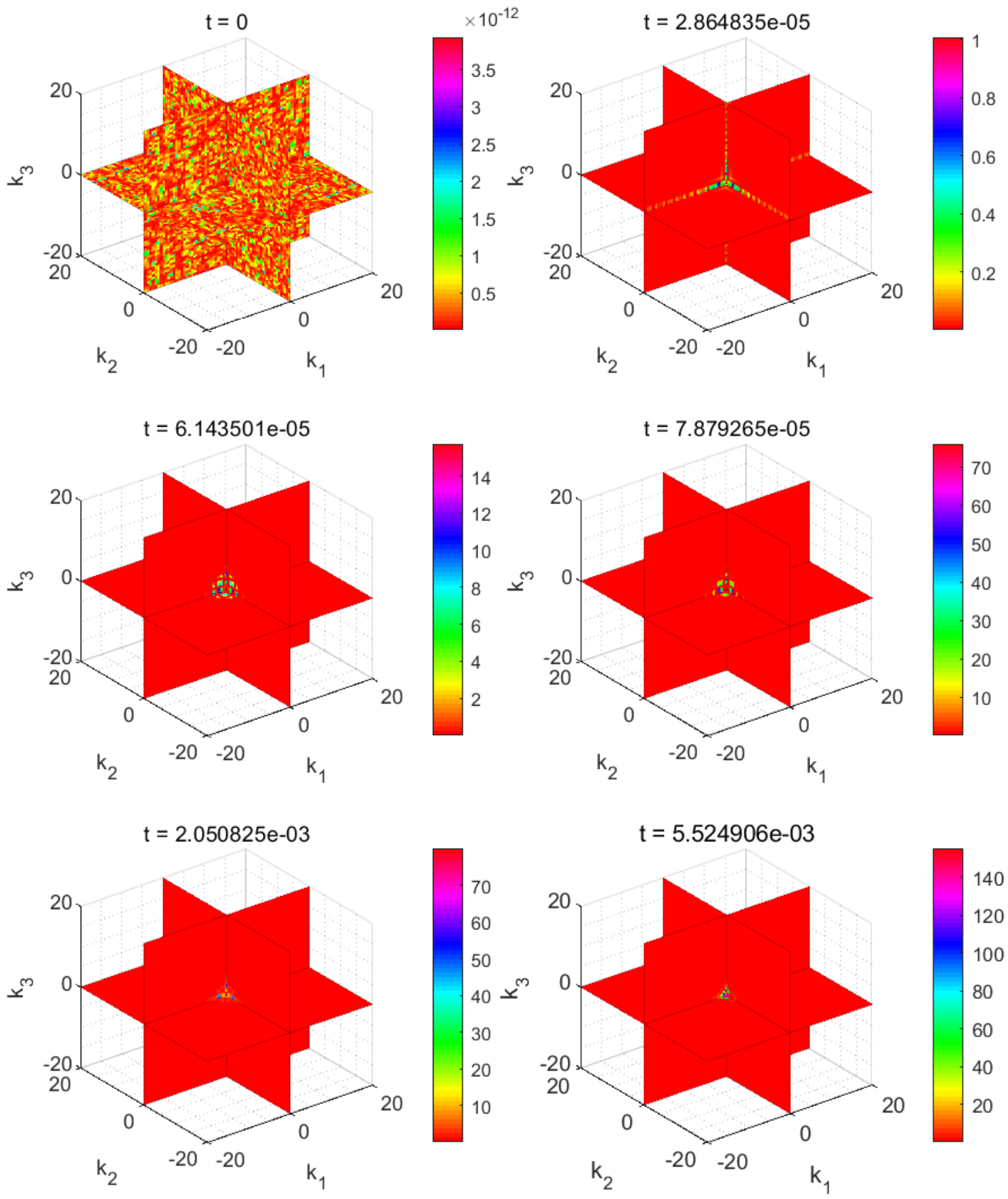

Figure 5.3-3 Structure factor of configurations shown in Figure 5.3-1 (Case 5.3.A) 

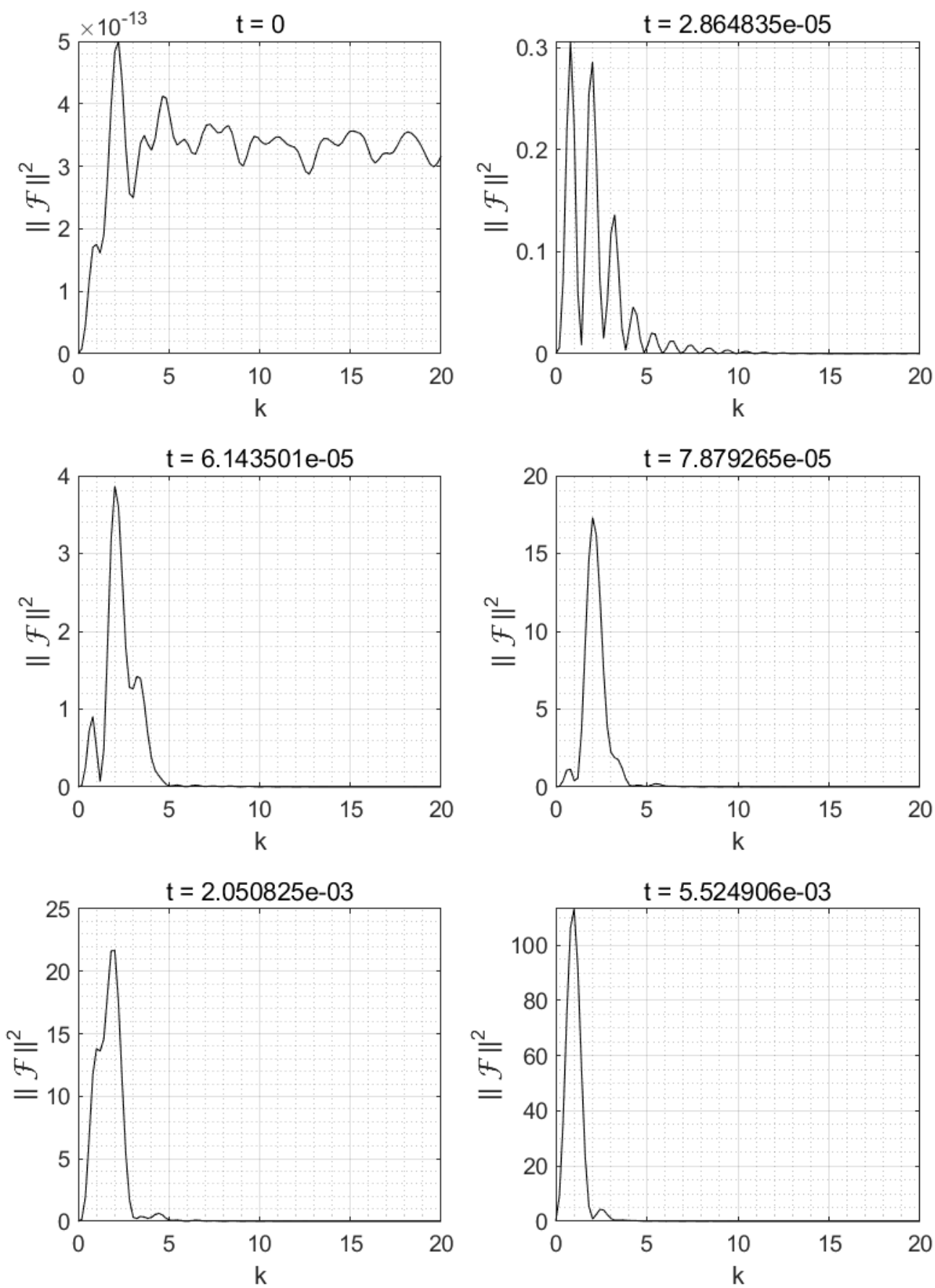

Figure 5.3-4 Circularly averaged structure factor of simulation in Figure 5.3-1 (Case 5.3.A) 

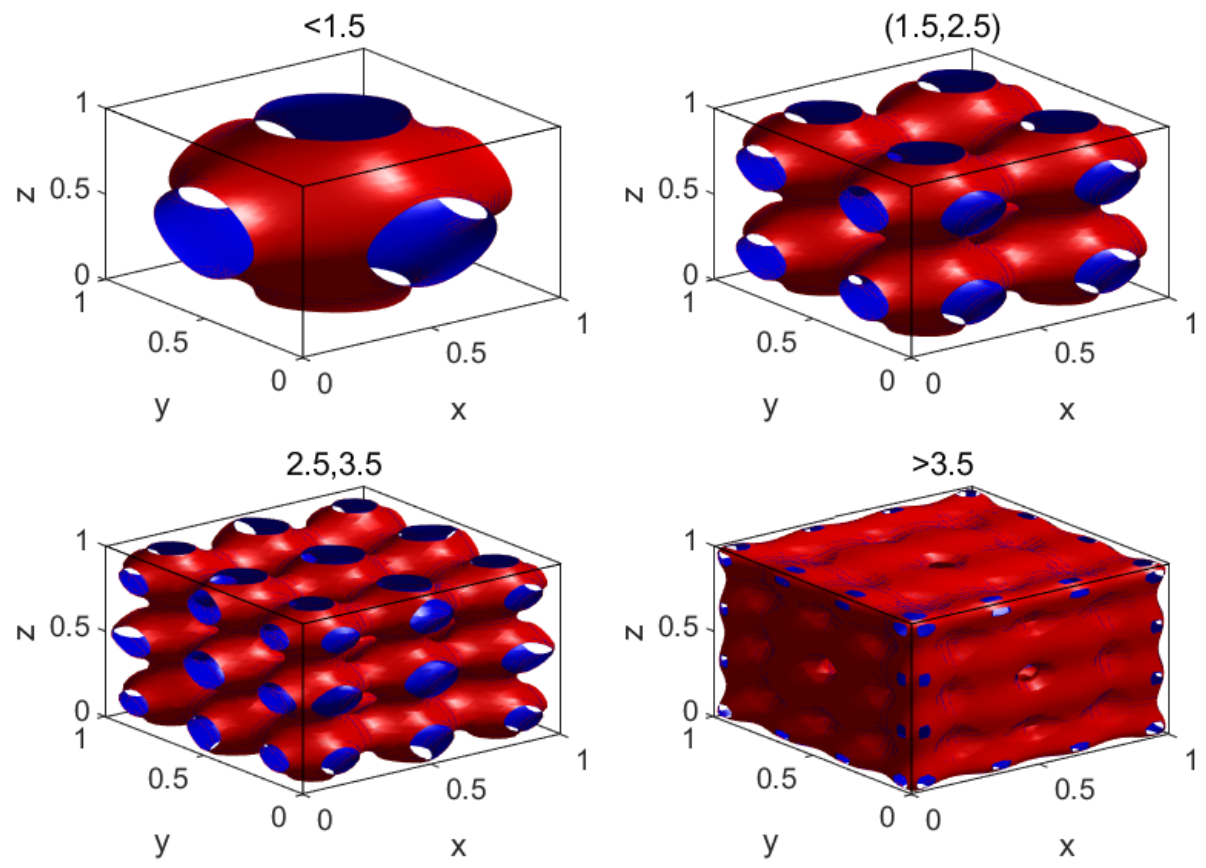

Figure 5.3-5 Isosurfaces of configuration in Figure 5.3-1 after band-pass filters are applied at $t=$ $2.864835 \times 10^{-5}$ (Case 5.3.A)

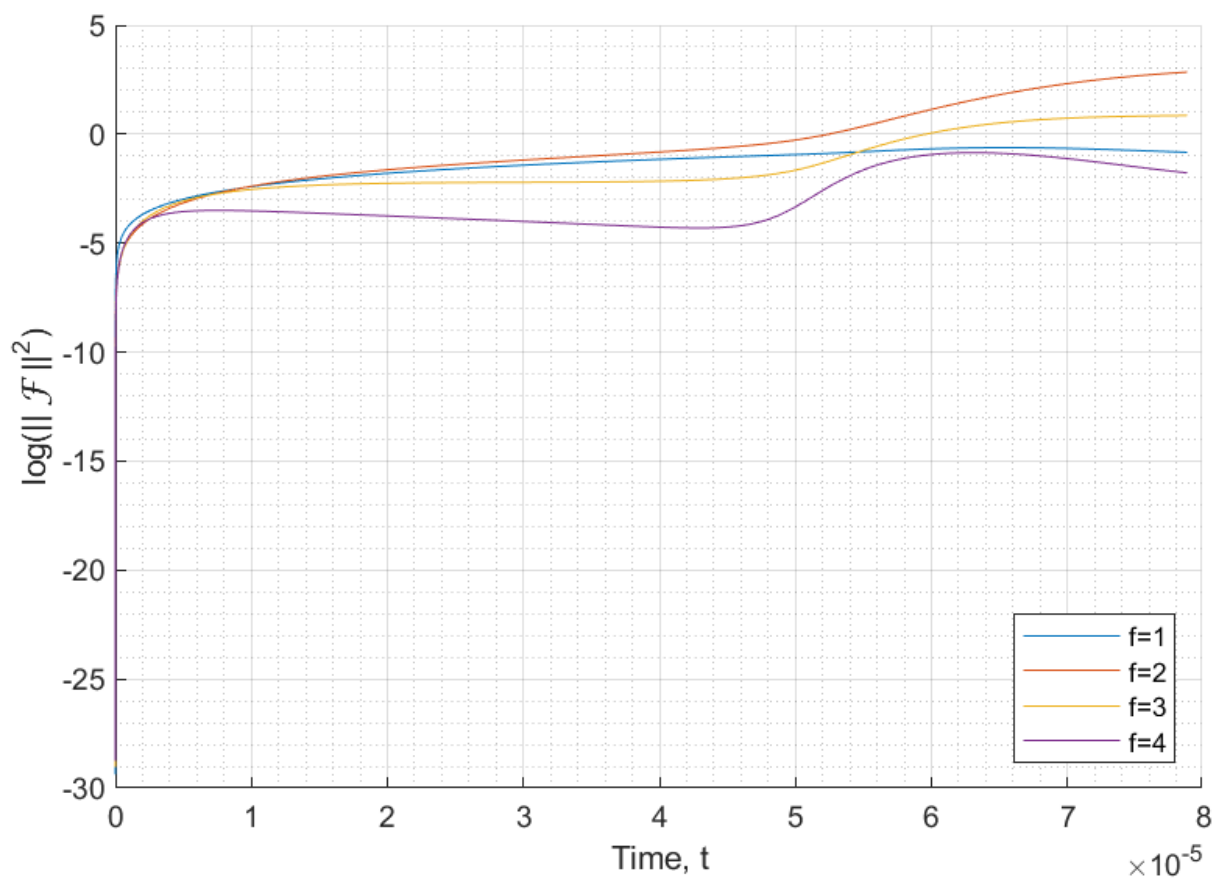

Figure 5.3-6 The amplitudes of structure factors at specific frequencies of the simulation in Figure 5.3-1 (Case 5.3.A) 


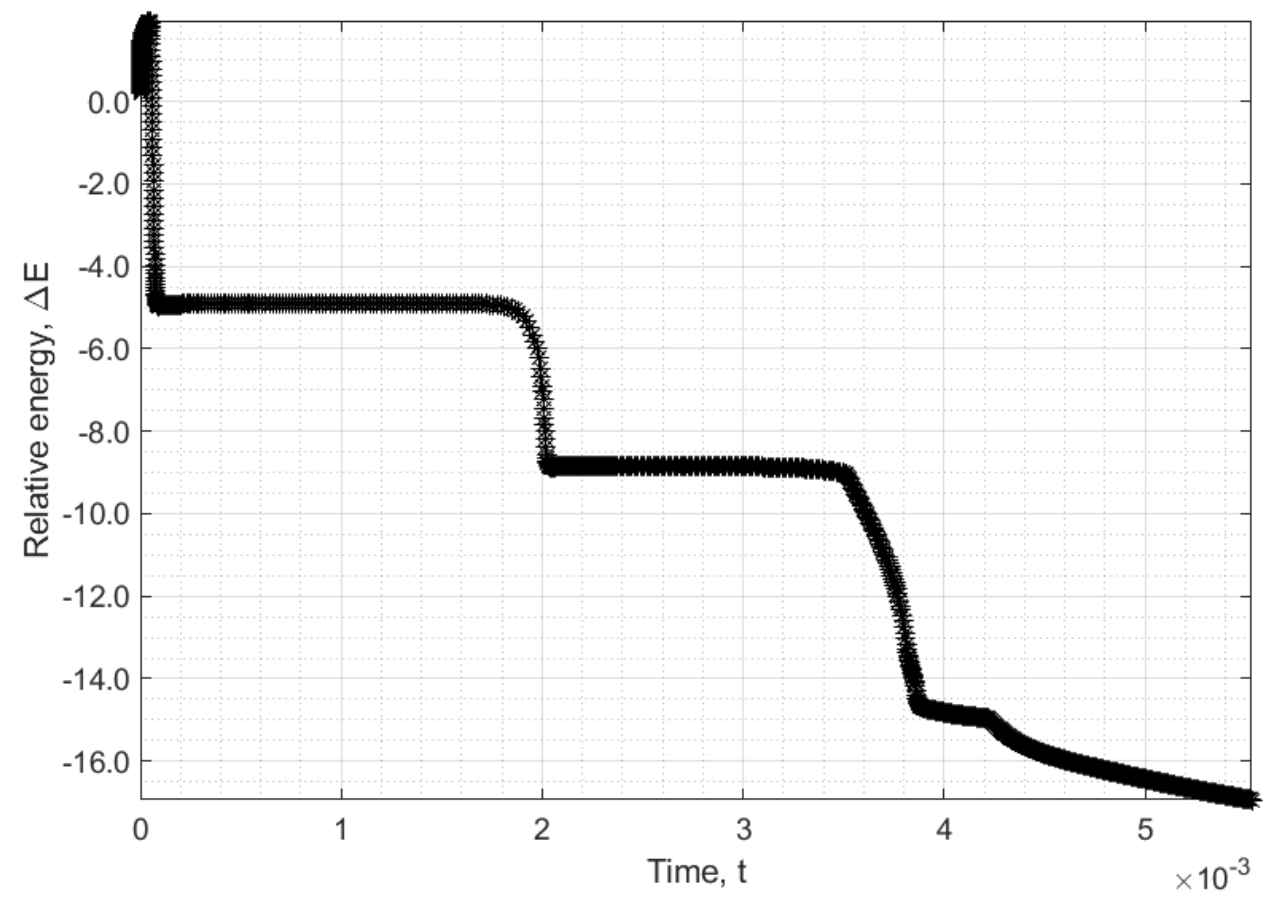

Figure 5.3-7 Relative energy of the process shown in Figure 5.3-1 (Case 5.3.A)

\subsection{Concentration gradient}

Preliminary results for concentration gradient has been established previously by Jiang and Chan [77]. Though not shown here, present model can replicate the results from previous studies easily. In this thesis, the case when the concentration gradient has crossed the spinodal curve is more of interest as the solution contains metastable region and unstable region. Here, in order to avoid potential energy density difference at the boundary, which may cause either of the component to become clumped at the boundary, large random initial noise, $\left\|\eta_{o}\right\|_{\max }=1.0 \times 10^{-3}$, generated by using randn function in Matlab, is used, and a concentration gradient that takes the form of third degree polynomial whose derivatives are zero at all boundaries in normal outward direction. For this chapter, 3 cases are considered based on average concentration among other parameters that would categorize the solution in metastable, unstable, or stable without initial fluctuation. 
First, metastable solution, based on the average concentration, is considered. In particular, a solution with $D=100000, T=0.35, n_{1}=n_{2}=1$ and concentration varies from $c_{1}=0.25$ at $x=0$ to $c_{2}=0.35$ at $x=1$ (Case 5.4.A) is considered. This way, the boundary that separates unstable and metastable region is slightly higher than $x=0.5$. Thus, the quench depth increases in increasing direction of $x$. Here, note that the average concentration is $c_{\text {ave }}=0.3$, thus the solution is in metastable region if uniformly mixed. The concentration profile and contour plots are shown in Figure 5.4-1 and Figure 5.4-2, respectively. Initially at $t=0$, increasing concentration is seen in positive $\mathrm{x}$ direction. The droplets are emerging at $t=1.735279 \times$ $10^{-7}$, near the boundary, where the quench depth is the deepest. The surrounding area of each droplet lowers in concentration. When it lowers below the average concentration, it creates eye-ball like pattern. This pattern then gradually shifts towards shallower quench side, bringing all regions except component-1 rich droplets lower than average concentration. At $t=$ $7.239773 \times 10^{-7}$, the boundary that separates the regions of higher and lower concentration is disappearing, the only regions that are component-1 rich are in the droplets. After this time, the simulation is let run for long time, until $t=2.376912 \times 10^{-6}$. Until this time, the droplets never appeared in the shallow regions. Meanwhile, the droplets that have appeared are experiencing late stage. The disappearing of small droplets and enlargement of neighboring droplets only happen in the deepest quench region, between $x=0.8$ and $x=1$. This mechanism is not seen in the leftmost droplets. Instead, they merge into larger droplets. Phase separation seems to occur only in deeper quench region near $x=1$ because this region's initial concentration has been raised to become locally unstable. On the other hand, phase separation, at least in the form of droplets, is not observed in the region near $x=0$ because this region is initially locally metastable and the fluctuation is not enough to initiate phase separation. However, as can be seen later, in later stage, the concentration in this region varies dynamically, i.e. it is affected by phase separation in locally unstable region. To analyze this result more quantitatively, structure factor of this simulation is shown in Figure 5.4-3. Initially, two peaks at about $k_{1}= \pm 0.8$ and $k_{2}=0$ is seen. This is the structural anisotropy initially created by concentration gradient. These two peaks seem to persist throughout the entire simulation range. Thus, concentration gradient apparently has very long term effect in low frequency structure. In fact, they are the dominant structures and increase throughout the process, as 
shown in Figure 5.4-8. Seemingly isotropic ring structure is seen most clearly (of all the time frames shown) at $t=3.734029 \times 10^{-7}$. This is the time frame when all the droplets will seem to have similar structure. As the simulation proceeds, lower-frequency droplets seem to emerge at $t=7.239773 \times 10^{-7}$. As the time proceeds further, high-frequency droplets, or smaller droplets, disappear while lower-frequency droplets become more apparent, as can be seen at $t=2.376912 \times 10^{-6}$. This is a typical structure in late stage. In order to understand which droplets account for what range of frequencies, band pass filters at $t=3.734029 \times$ $10^{-7}$ and $t=7.239773 \times 10^{-7}$ are shown in Figure 5.4-4 and Figure 5.4-5, respectively. Lower frequency structure, $r<3$, are anisotropic and they seem to vary only in $\mathrm{x}$ direction and a lot less in y direction. The higher the band-pass filter range is, the droplets are found more in deeper quench region. In order to better illustrate this, concentration variance along $\mathrm{x}$ direction is computed. It shows how vigorous the concentration fluctuate along $\mathrm{x}$-direction. It is computed by calculating the variance of concentration along $y$-direction at each coordinate of $x$. However, the reader must be aware of the limitations. As the droplets are not always nicely aligned in the similar way, some values will not be a good representation of the fluctuation. For example, if a vertical line (parallel to $y$-axis) drawn at certain $x$-value does not contain any droplets at all, by some chance, then the data is less reliable. Nonetheless, it still gives a reasonable overall evaluation of anisotropy within the domain. Figure 5.4-6 and Figure 5.4-7 show concentration variance at $t=3.734029 \times 10^{-7}$ and $t=7.239773 \times 10^{-7}$, respectively. At $t=3.734029 \times 10^{-7}$, concentration variance of highest-frequency structure, $\left(r_{1}, r_{2}\right)=$ $(12, \infty)$ is most apparent on the deepest quench region. Most remarkably however, is the variance of the structure $\left(r_{1}, r_{2}\right)=(8,12)$. These structures seem to be spread through the region that contain droplets, especially in the region between $x=0.7$ and $x=0.95$. Recall that, this is the time frame when spinodal-ring, as shown in Figure 5.4-6 is the sharpest. Thus, similar morphology is observed throughout the entire region that has droplets. Later, at $t=$ $7.239773 \times 10^{-7}$, a rise of structure of $\left(r_{1}, r_{2}\right)=(3,8)$ is seen, especially at the highest around $x=0.68$. This is where the newest and the last droplets will emerge. These last set of droplets thus has a unique structure that is different from the majority of the droplets. Also note that, at this points, structural an anisotropy has been established: higher frequency structures are more concentrated towards the deepest quench region. Finally, in order to 
investigate the overall process of the solution, the mean concentration along $x=0$ is monitored throughout the entire simulation range. Because the solution behaves as a whole by mass conservation, and also because the minimum energy is reached only when the entire solution is at the equilibrium values for unstable and metastable solutions, the behavior of shallow region cannot be ignored. The mean concentration along $x=0$ is shown in Figure 5.4-9. It can be seen that, the concentration increases continuously until about $t=2.1 \times 10^{-6}$. This is because, at first, this region mostly behaves locally. That is, since the concentration in this area is mostly within unstable region, any high frequency noise is quickly eliminated, and the solution tries to go into uniformly mixed state. Because the concentration is higher in the inner region, the concentration tends to flow towards shallowest quench boundary, raising to concentration. However, after $t=2.1 \times 10^{-6}$, the solution then starts decreasing. As the droplets in the deep quench region are stabilized, they lowered the concentration of the matrix, thus creating a wave that propagates towards shallowest quench side. Thus, this decrease can be thought of as the wave reaching the end of the domain. At this point, the solution no longer interacts only locally, and rather dynamically as component- 1 and component- 2 attempt to reach equilibrium values of $e_{1}=0.1839$ and $e_{2}=0.8161$, respectively. Thus, if the simulation is to proceed further, one can expect to see the concentration approaching close and closer towards $e_{1}=0.1839$ in Figure 5.4-9, while the droplets on the deeper quench sides to merge into macro-spheres with the peak at about $e_{2}=0.8161$. At this stage, the solution has phaseseparated and will not go back to its uniformly mixed state. The overall change in relative energy, as shown in Figure 5.4-10, shows that the solution goes to zero negative relative energy, thus the solution will remain phase separated as it has achieved a more favorable state. A lag time, or the duration in which relative energy changes slowly, is seen from the start to about $t=1.8 \times 10^{-7}$. This is the period when the solution is conforming to thermodynamically favorable morphology. Because concentration gradient is huge, this re-arrangement behaves differently in separate regions, i.e. droplet shape forms in deeper quench region, while the solution becomes more uniformly mixed in shallow quench region. After this, the droplets in deep quench region merges quickly. Thus, it has been shown that, by generating concentration gradient so that one region becomes temporarily unstable, metastable solution can still undergo phase separation. 

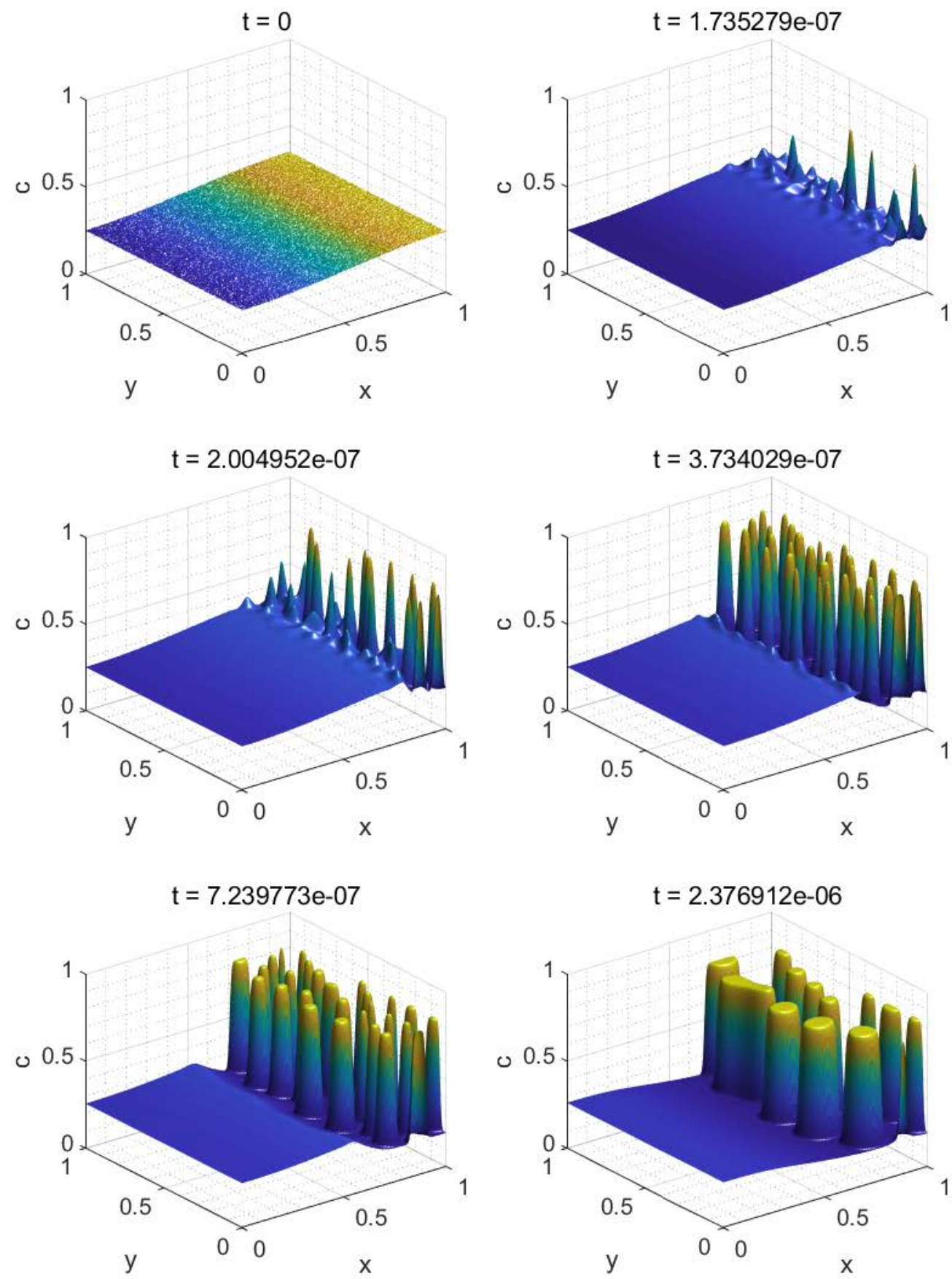

Figure 5.4-1 Concentration profile of the solution with $D=100000, T=0.35, n_{1}=n_{2}=1$. Initial concentration varies from $c=0.25$ at $x=0$ to $c=0.35$ at $x=1$ and also contains noise. (Case 5.4.A) 

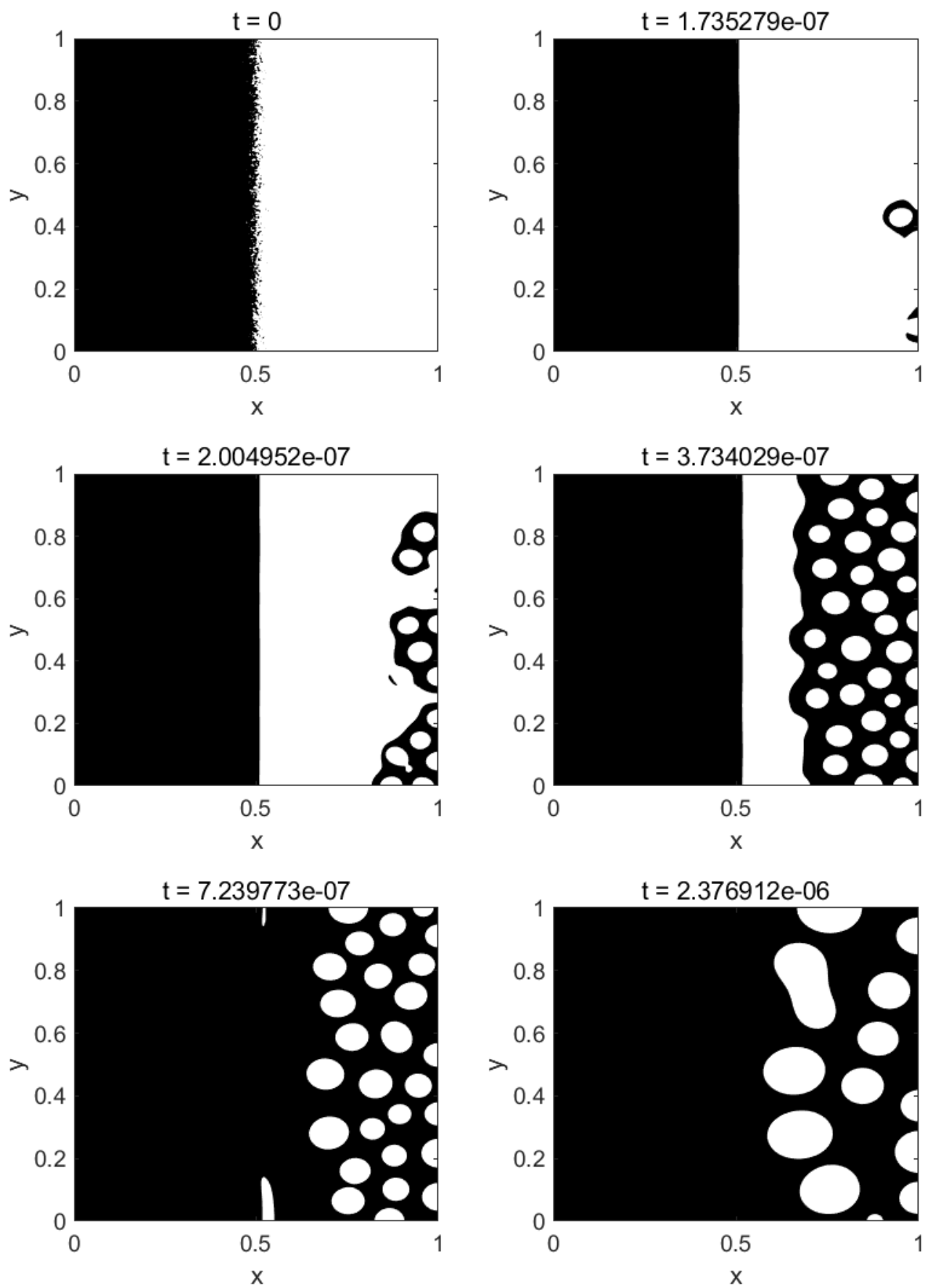

Figure 5.4-2 Contour plots of the simulation in Figure 5.4-1 (Case 5.4.A) 

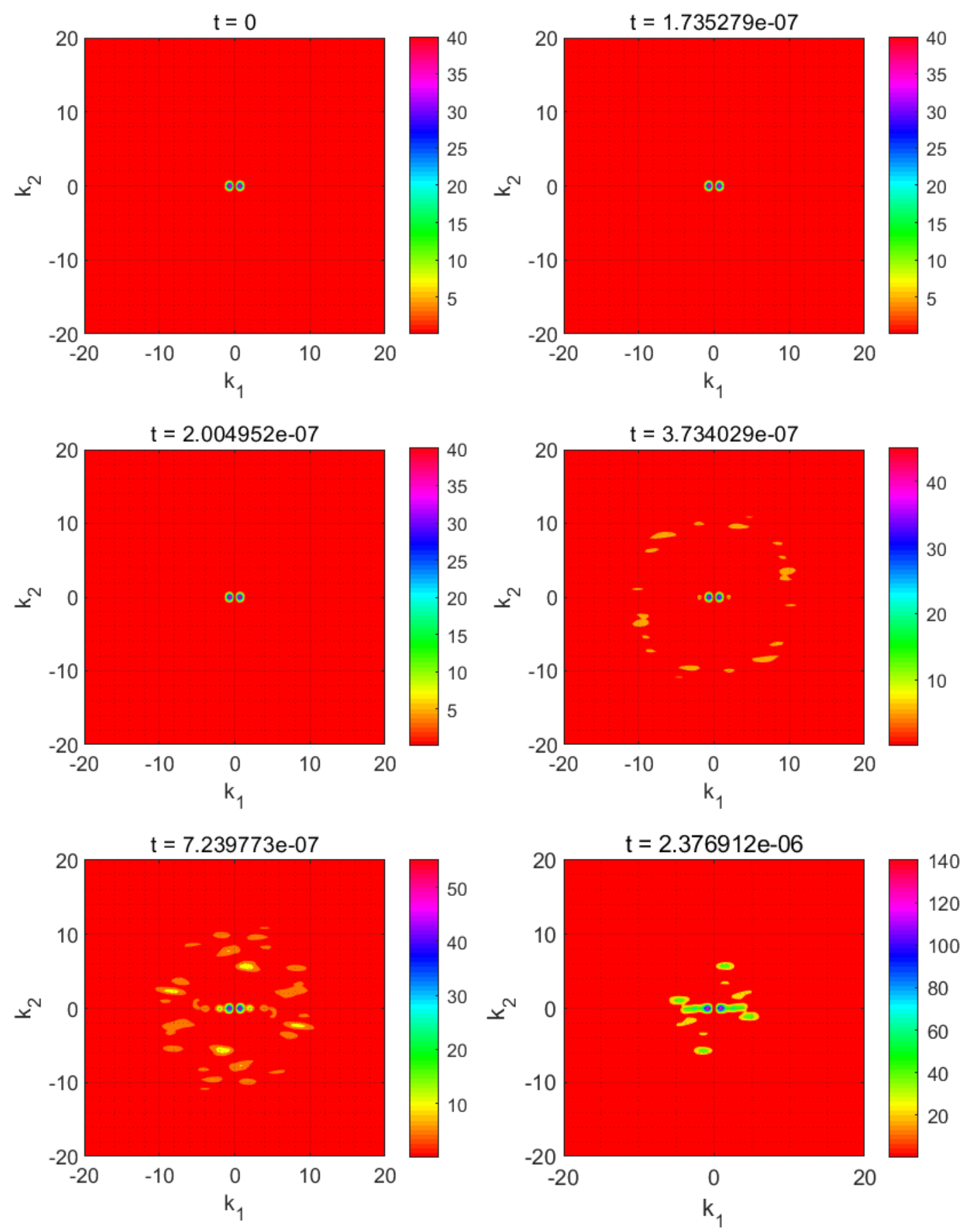

Figure 5.4-3 Structure factor of the process in Figure 5.4-1 (Case 5.4.A) 


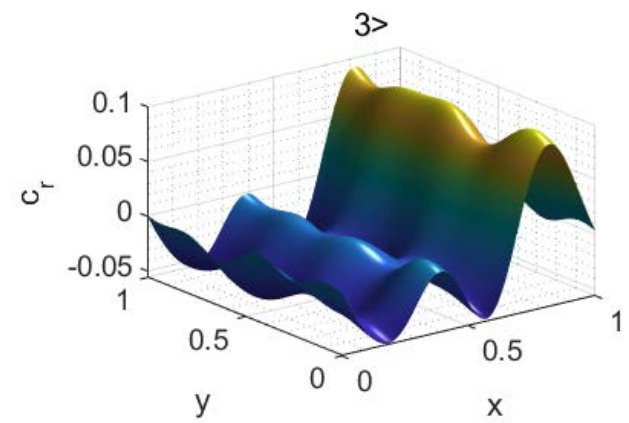

$(3,8)$
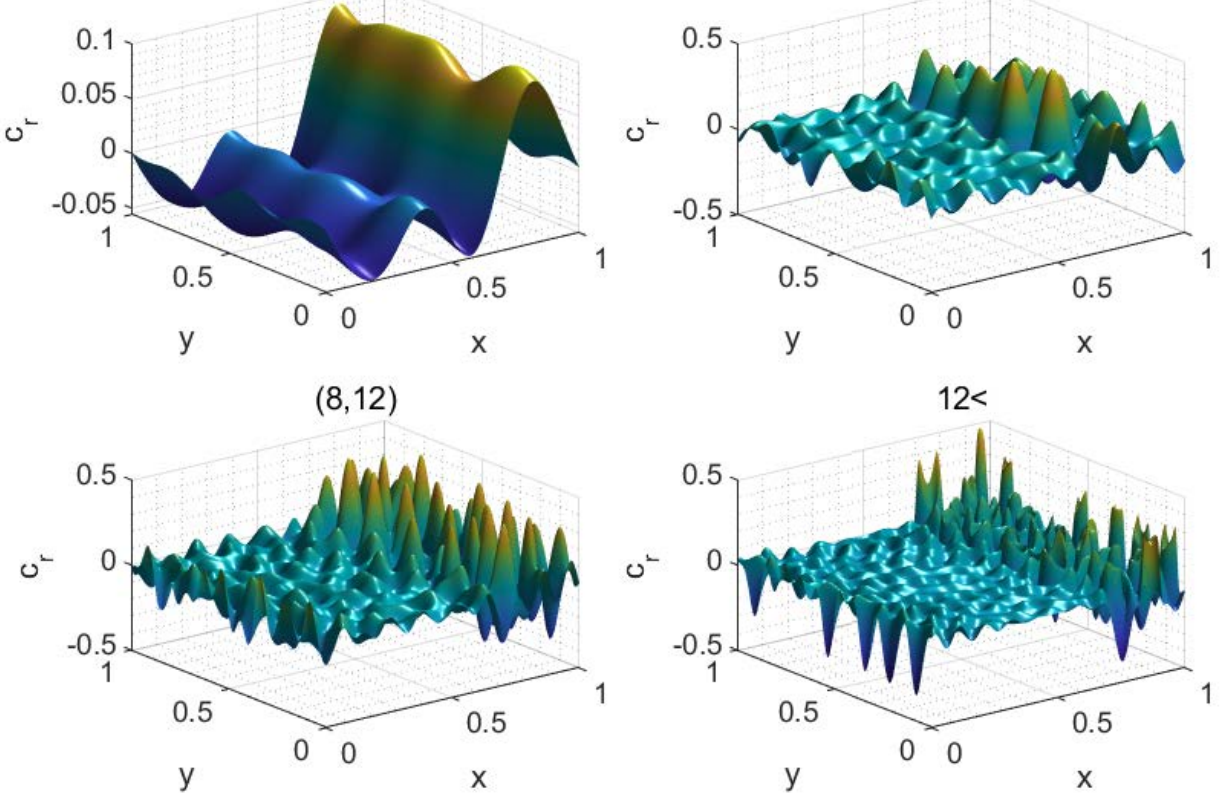

Figure 5.4-4 Band-pass filters of configuration at $t=3.734029 \times 10^{-7}$ in Figure 5.4-1 (Case 5.4.A)

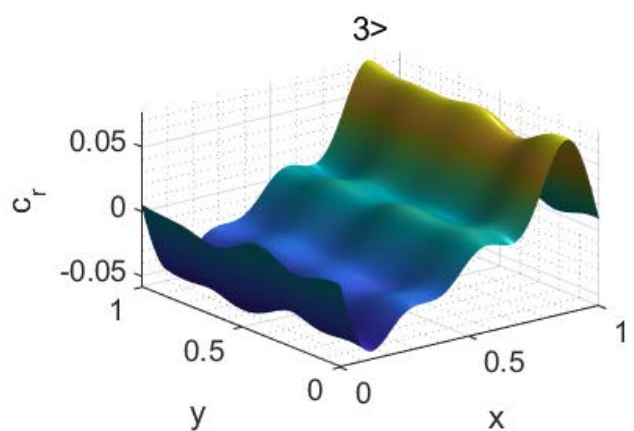

$(8,12)$

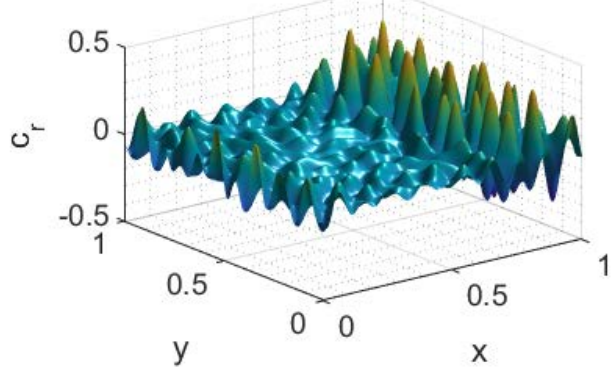

$(3,8)$

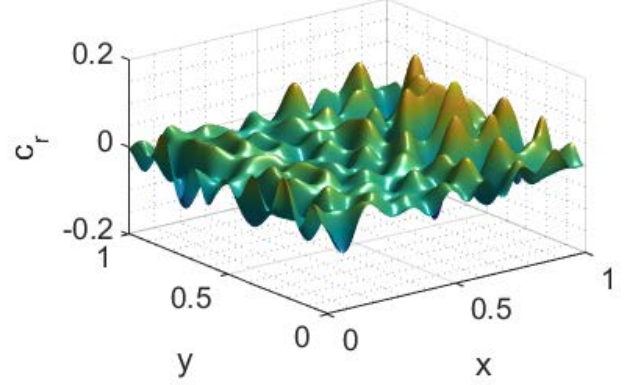

$12<$

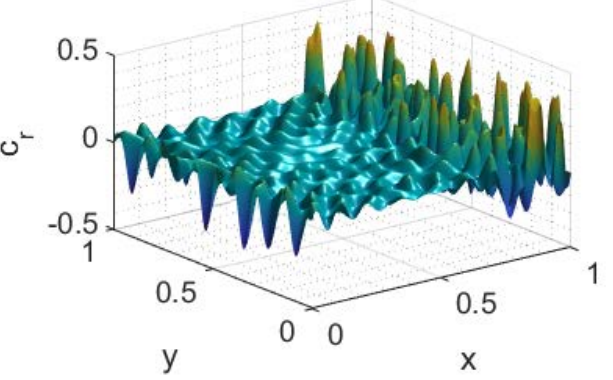

Figure 5.4-5 Band-pass filters of configuration at $t=7.239773 \times 10^{-7}$ in Figure 5.41 (Case 5.4.A) 


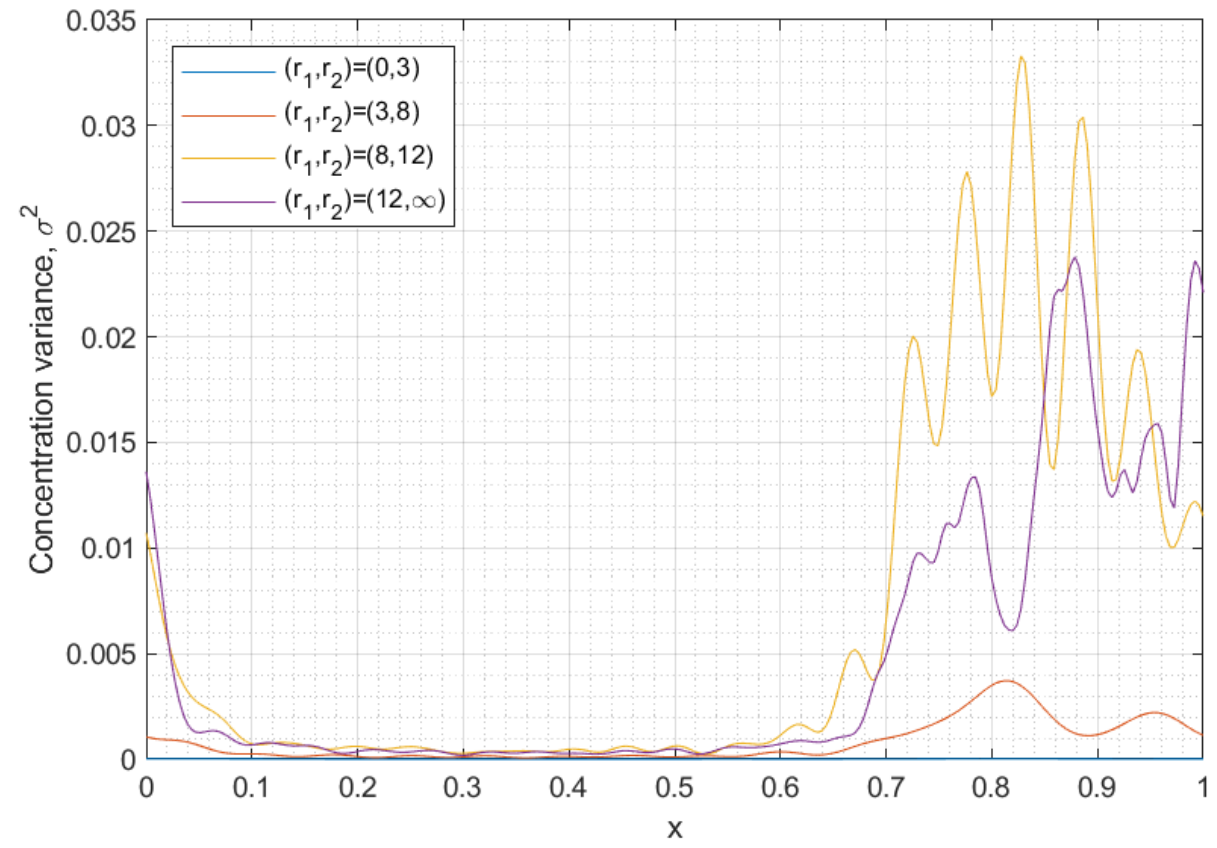

Figure 5.4-6 Concentration variance of configuration at $t=3.734029 \times 10^{-7}$ of the same simulation as in Figure 5.4-1 (Case 5.4.A)

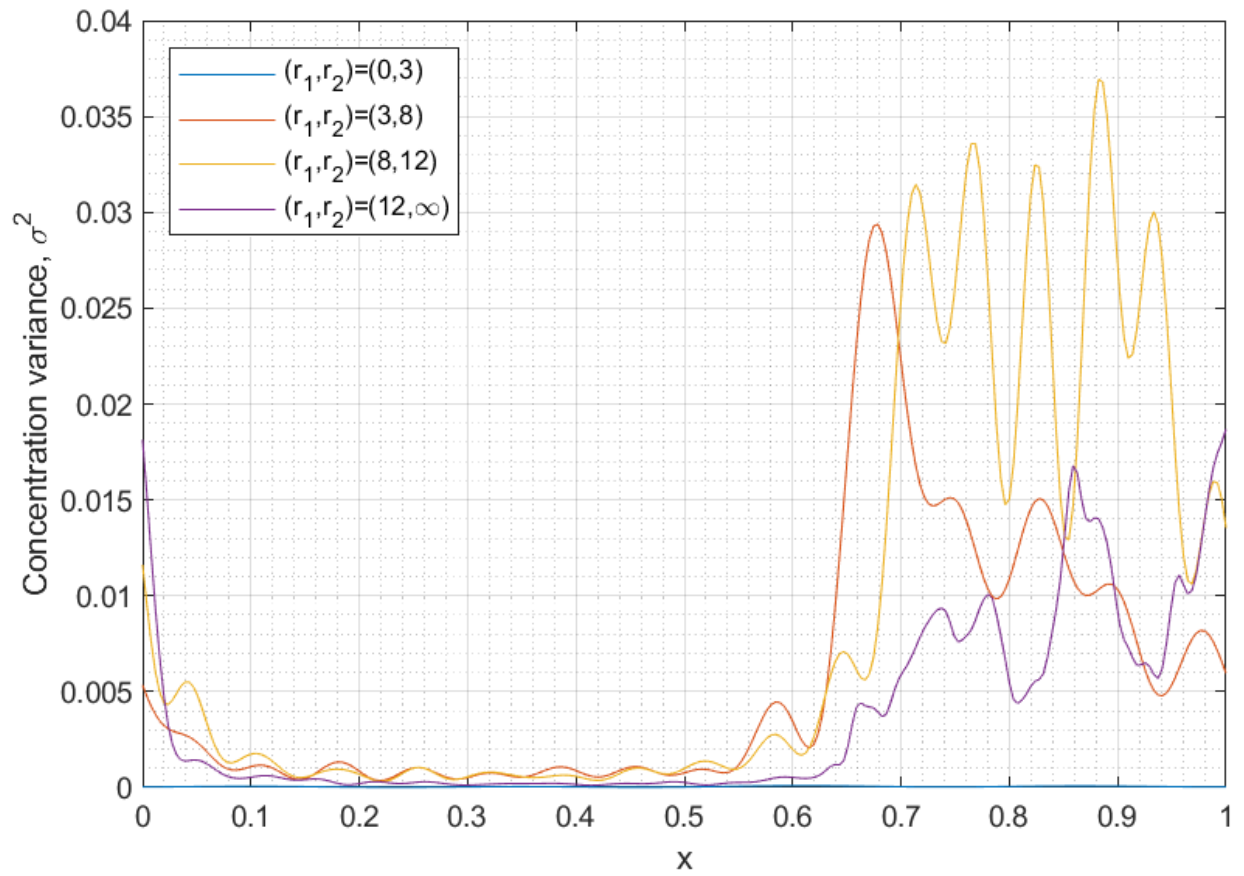

Figure 5.4-7 Concentration variance of configuration at $t=7.239773 \times 10^{-7}$ in of the same simulation as in Figure 5.4-1 (Case 5.4.A) 


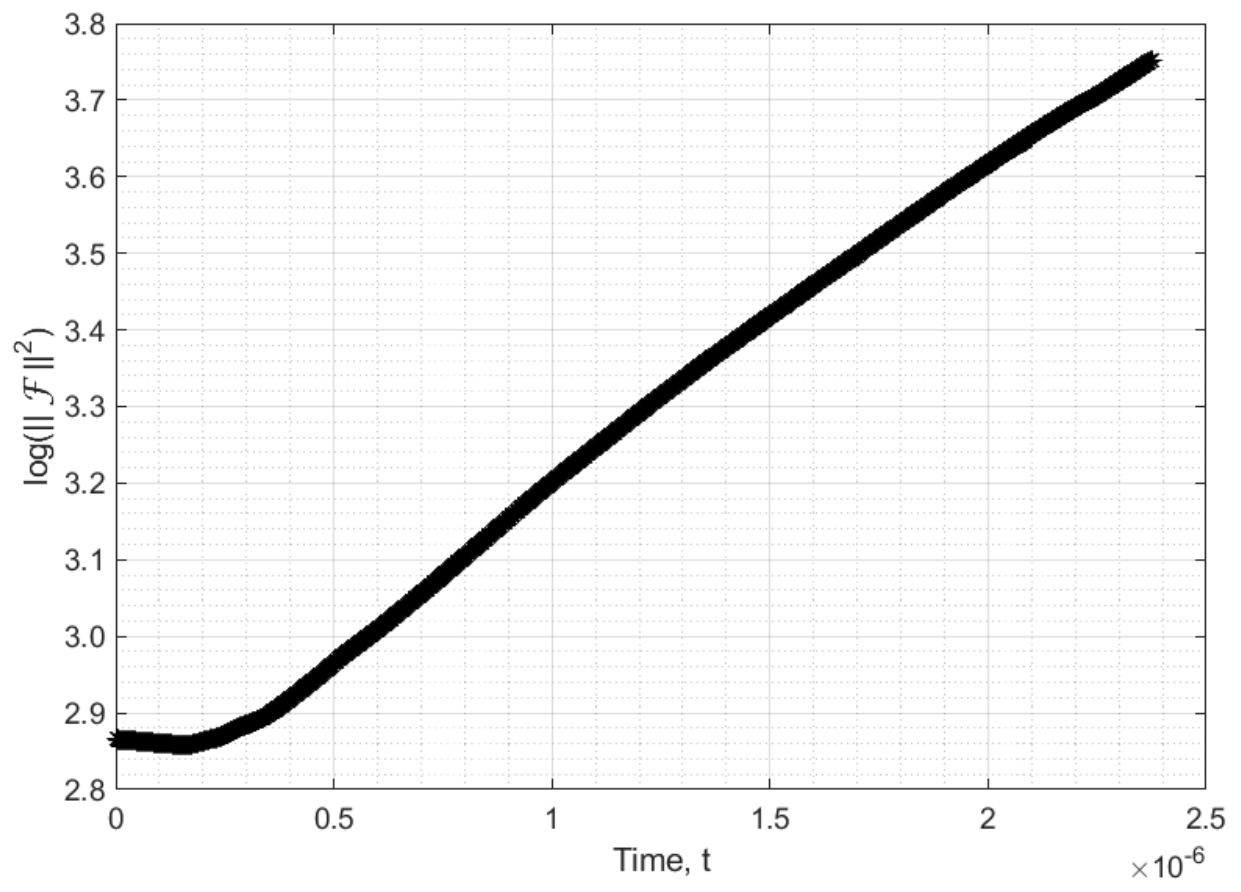

Figure 5.4-8 Maximum structure factor of the solution in Figure 5.4-1 (Case 5.4.A)

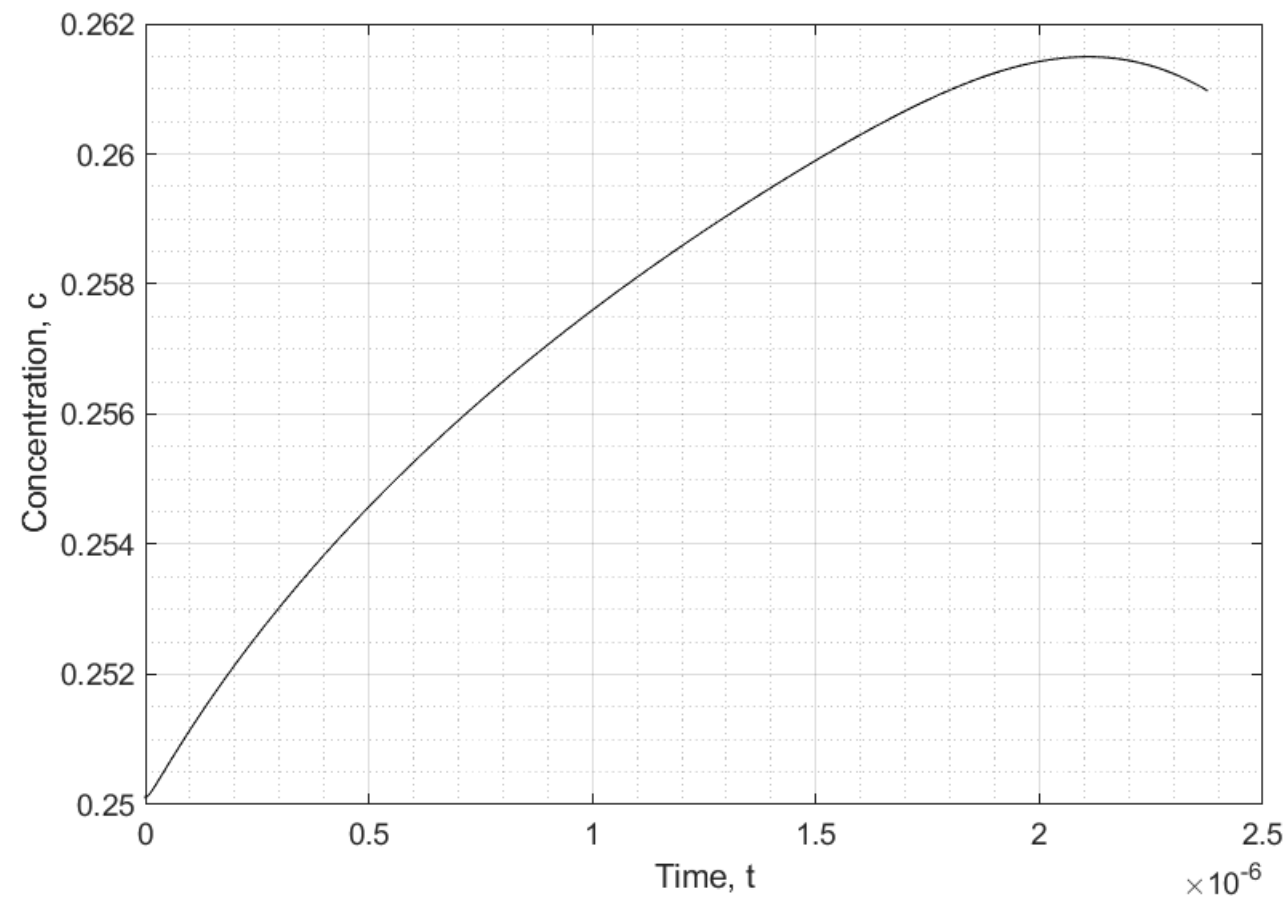

Figure 5.4-9 Average concentration along $x=0$ in the simulation in Figure 5.4-1 (Case 5.4.A) 


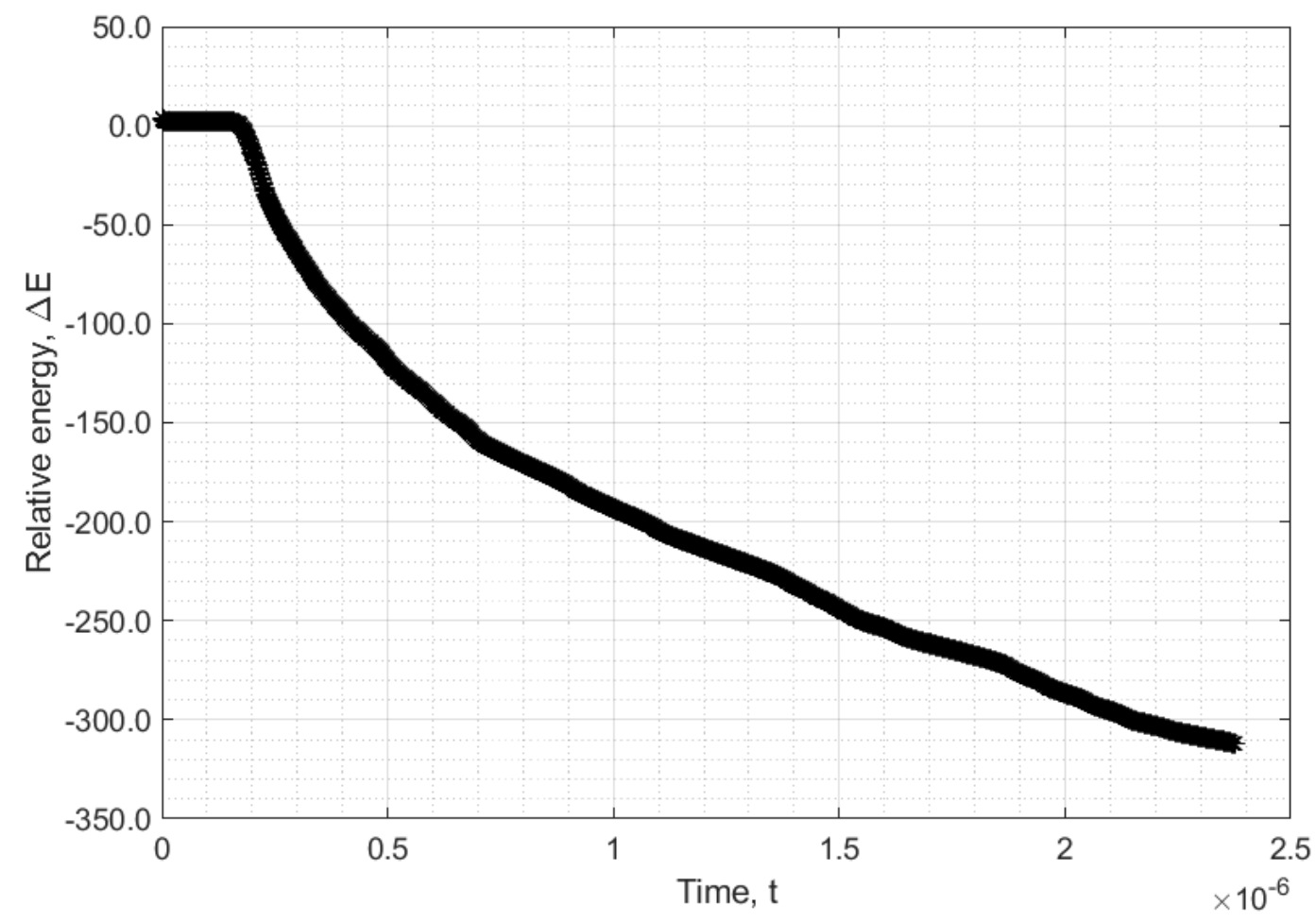

Figure 5.4-10 Relative energy of the process in Figure 5.4-1 (Case 5.4.A)

\subsection{Spinodal decomposition on the complex domain with curved boundaries}

All of the studies so far are done on a square domain of area of 1 unit squared. At this point, readers are reminded that this study is based on non-periodic boundary conditions. In other studies with periodic boundary conditions, the structures at one end continue to another end. In non-periodic boundary conditions, the solution behave somewhat differently from the major portion of the solution because of wetting and no mass flux boundary conditions. Since square domain is conveniently chosen, much of numerical methods are simplified, as explained in more details in Appendix A. Since the effects from the boundaries are apparent and should not be ignored, and because spinodal decomposition that occur in nature is not expected to occur on a conveniently square domain, one should be able to readily adapt the model to make it more realistic. For this reason, there are several studies of spinodal decomposition already 
done on complex geometries. In this section, it is demonstrated that, if needed, present model is appropriate for simulating the process on domains with complex geometries. More detailed numerical method and its advantages and disadvantages are mentioned in Appendix A. In short, because need to apply wetting boundary conditions, the basis functions at the boundaries are twisted so that they represent the nodal weights of the element in such way that one basis function within an element represent the first directional derivative normal to the surface, while another represents the first directional derivative tangent to the surface. The basis functions that are not at the boundary are also twisted appropriately. Because of the lack of experimental studies on a confined domain with complex geometry, this chapter solely serves as the numerical demonstration.

To the best of the author's knowledge, there has not been numerical study of surface directed spinodal decomposition with circular (or spherical for 3-d) domain. The trick of such study lies in wetting boundary condition, which requires that the first derivative of the concentration with respect to the normal of the surface must be at certain values. Present model is appropriate for such, because the numerical method specifically determines the weights of the derivative of the concentration at any desired direction. For neatness, this method is outlined in Appendix A. To demonstrate this in the simplest form, circular domain with the area of 1 unit squared is selected as demonstration. Spinodal decomposition is performed with the following parameters: $D=100000, c_{\text {ave }}=0.5, T=0.3, n_{1}=n_{2}=1$. The wetting boundary condition is applied for constant $h$, and for simplicity, it does not depend on the concentration. The overall processes are shown as concentration profile in Figure 5.5-1 and as contour plots distinguishing phases in Figure 5.5-2. Generally, when $h=0$, spinodal decomposition proceeds as one would expect in the a square domain. The result is a randomly distributed interconnecting structures within the domain. The morphology does not seem to vary across the domain. Visually, the closer look of concentration profile shows that, indeed, the concentration gradient becomes zero in the direction normal to the domain at the boundaries at all time. Thus, present numerical method is successful for curved boundaries. To strengthen the model, the values of $h$ on the entire circular boundary is gradually increased for different cases. Since the boundary condition is perfectly symmetrical from all directions, simulations on 2-d mesh is appropriate. 
As $h$ is increased, the interconnecting structures become less random. At a moderate value of $h=10^{-5}$, macrostructures with concentric shape emerge. Within each ring, there exists smaller wave-like structures that arise like droplets. In the latest frame, the convoluted circular arcs result. For highest $h$ values tested, the wave structures disappear very soon. The ring of high concentration at the boundary emerges quickly, followed by a well-defined arc region of low concentration. This pattern propagates inwards, leading well-structured target or concentric pattern.

The results observed for circular domain is quite analogous to square domain case: For sufficiently strong wetting effect, the fluctuation within the solution tends to align with the shape of the boundary because the concentration fluctuation adjacent to the boundary is the quickest to emerge. The result is, a well-organized morphology that is directed strongly by the shape of the boundary. This section serves to demonstrate that present model can effectively make numerical study of phase separation of multi-phase polymer solution possible with and without wetting. Because the shape of the domain can influence the course of phase separation, it is then, likely possible that one can achieve desired morphology by altering the shape of the domain by designing complex geometries. Here, it is left as suggestion for potential future projects. 


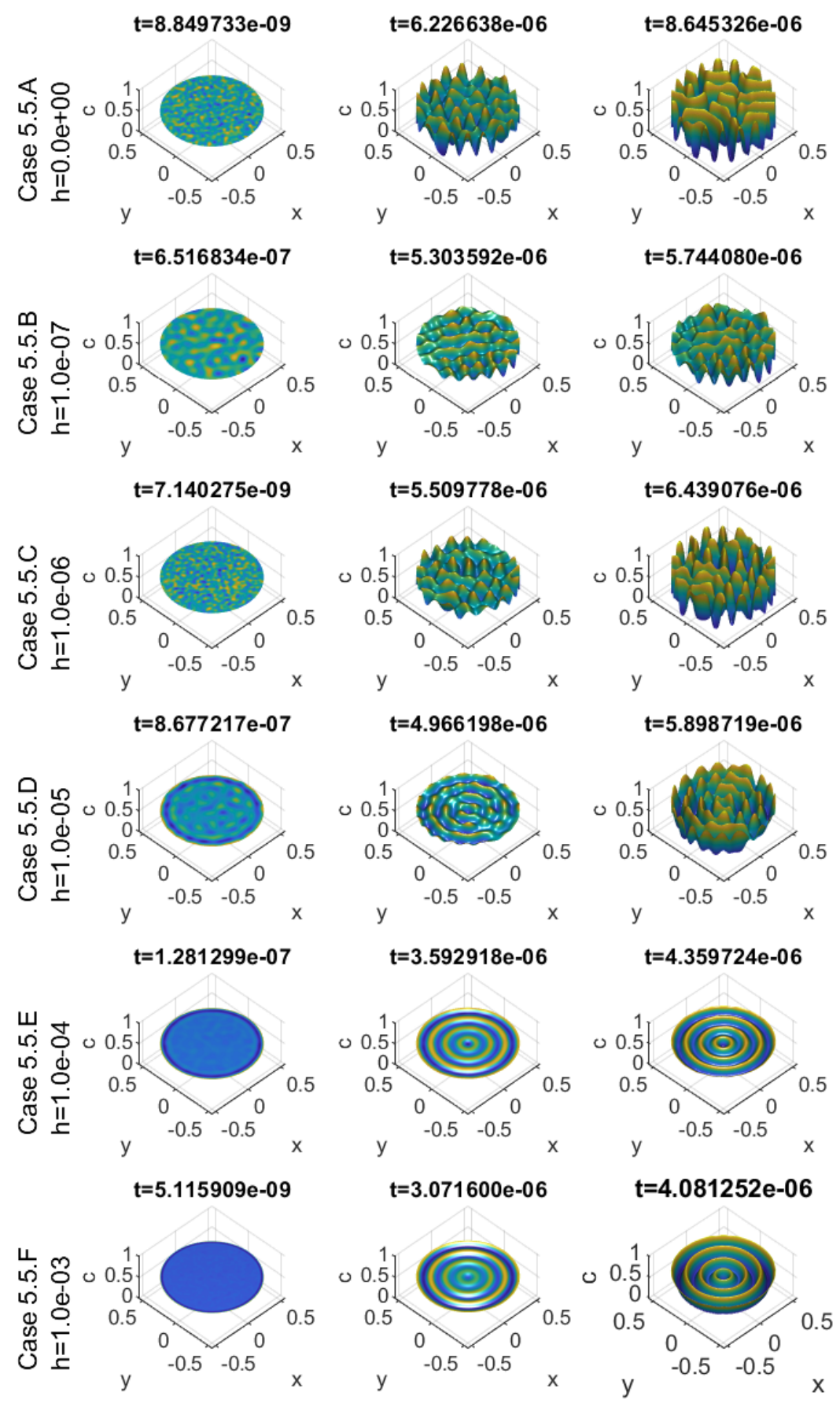

Figure 5.5-1 Concentration profile of $D=100000, c_{\text {ave }}=0.5, T=0.3, n_{1}=n_{2}=1$ with different $h$ values. 

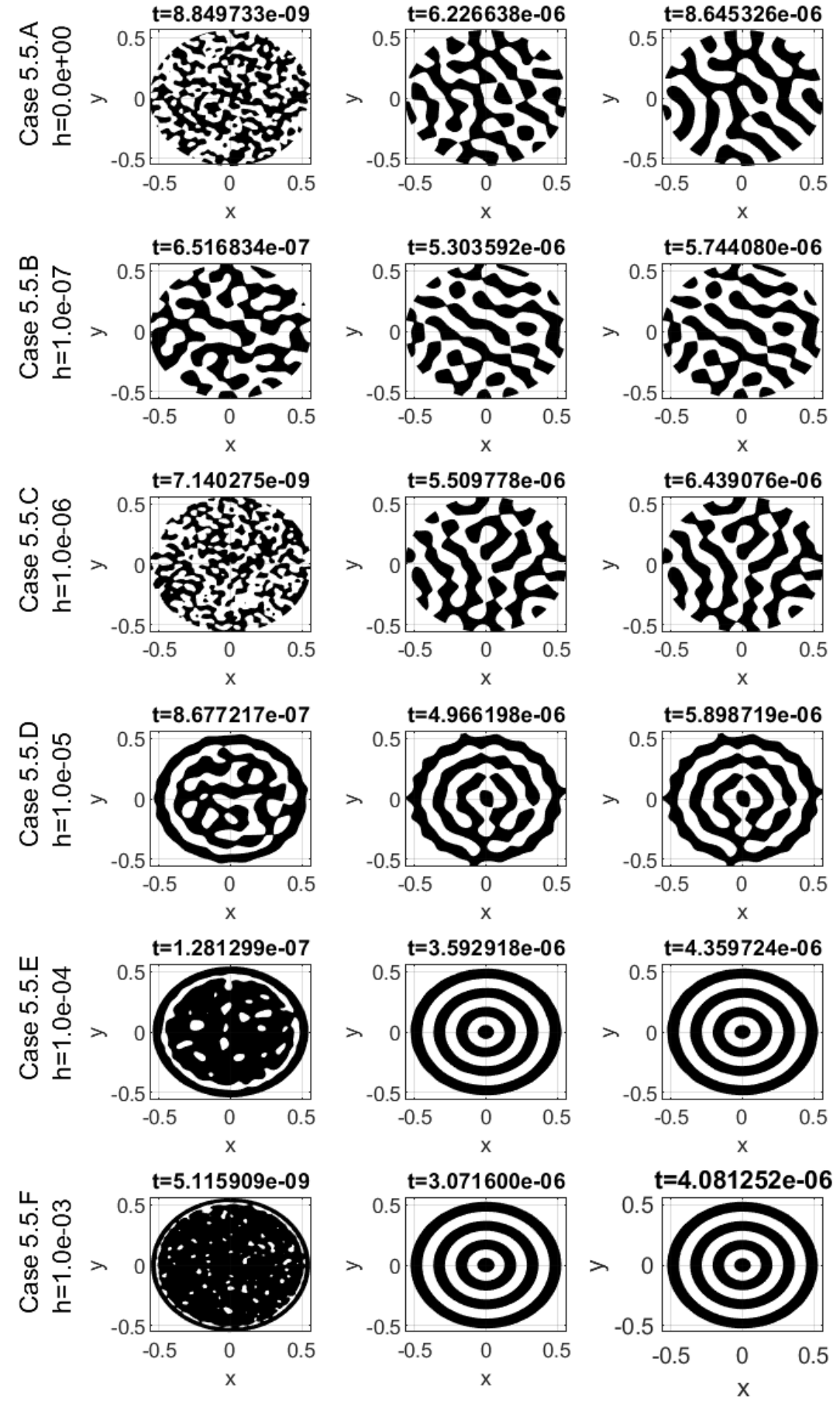

Figure 5.5-2 Contour plots of $D=100000, c_{\text {ave }}=0.5, T=0.3, n_{1}=n_{2}=1$ with different $h$ values. 


\section{Chapter 6 Conclusion}

In this thesis, variety of methods are shown to make phase separation in metastable solution possible. The noise was not included, except random initial fluctuation of the solution at the beginning, thus metastable phase separation from uniformly mixed state is impossible with single quench. Thus is good for the intentions of this thesis, because strategic phase separation

of metastable solution is of more interest, i.e. phase separation with the external influence.

Double quenching from unstable region to metastable region will either force the solution to go back to its uniformly mixed state or allow the phase separated structure to persist with new morphology and equilibrium values. Absolute energy of the solution in uniformly mixed state and its theoretical minimum energy can be determined using phase diagram and Cahn-Hilliard equation. By deliberately computing these, phase separation is guaranteed in metastable region if the first quench yields the configuration that has zero or negative relative energy at the onset of second quench. Otherwise if the relative energy is positive, characteristic structure factor is quickly eliminated while there is a little rise in the structure factors at low frequencies. Upon the drop in characteristic structure factor, if the solution does not drop to relative energy of zero soon enough, the solution will go back to its uniform state. If zero relative energy is reached quickly, on the other hand, the droplets will conform to new morphology and grow.

Aside from metastable quench, some of interesting morphologies that may be obtained with present model were generated. Remarkably, when double quench is carried out by varying polymerization of component 2 , if the timing and the parameters are right, one may be able to obtain a completely new random structures, concentric patterns, or cell-wall like structures. The later two are due to the direct influence and the persistence of already established droplets. The new fluctuations are influenced significantly by established droplets, causing the fluctuations surrounding it, thus forming an arcs around it. 
Anisotropic quenching can give graded structures in the solution. By combining multiple gradients, such as temperature and polymerization at the same time, a biaxial gradient can be produced. Readers should be aware, however, that numerical results obtained by polymerization gradient can be difficult to obtain in experimental settings because the process is usually accompanied by other influences such as volume shrinkage, polymer entanglements, elasticity, etc.

Metastable phase separation with wetting is possible. By taking advantage of growing fluctuation induced by preferential attraction of the domain, it may lead to the point where such fluctuation acts as trigger, or nucleation, for the growth to occur spontaneously. In the absence of spinodal decomposition, random high-frequency noise is quickly eliminated. Thus, if the preferential affinity is made the same in all boundaries, the droplets of almost perfectly symmetricity can be obtained. Only until the later stage, as the droplets merge, the symmetricity is destroyed.

Concentration gradient can also help to induce phase separation in solution whose average concentration would put it in metastable region. There seem to be 2 stages to it: at first, the fluctuations interact locally, i.e. spinodal decomposition takes place within unstable part of the solution, while high frequency noises are quickly eliminated in metastable part of the solution and this region slowly approaches average concentration, as high concentration flows down concentration gradient. After some time, structural anisotropy is established within the regions with the droplets. As the time proceeds, the solution acts more dynamically rather than locally: The metastable region now attempts to reach equilibrium value of the entire solution, while droplets emerged started to coarsen.

Present numerical method can be used on the curved boundary. Because the wetting can influence the morphology of the solution, it will be interesting to learn the behavior in a complex geometry in the future.

In summary, there are variety of methods to induce metastable growth in a polymer solution. It is hoped that some of these numerical results will inspire future experimental researches. In the future numerical studies, it is suggested that some other factors such as elasticity is 
included in the model. Also, the model did not include a "stopper" to freeze the morphology, thus the morphology obtained here are usually limited to different stages of local points in the solution. This, for example, may result in larger droplets in the deeper quench region of anisotropic structures because they enter late stage earlier. It is not yet possible, with present model, to achieve beginning of the intermediate stage at the same throughout the entire solution, though it may give some interesting graded morphology. Tran-Cong et al has utilized photo-cross-linking of the polymer to freeze the morphology, thus they were able to obtain graded co-continuous structures. While simple model is good to study isolated effect, more realistic model may better replicate experimental observations. 


\section{References}

[1] Cahn JW, Hilliard JE. Free energy of a nonuniform system. I. Interfacial free energy. The Journal of chemical physics. 1958 Feb;28(2):258-67.

[2] Toral R, Chakrabarti A, Gunton JD. Numerical study of the Cahn-Hilliard equation in three dimensions. Physical review letters. 1988 May 30;60(22):2311.

[3] Gómez H, Calo VM, Bazilevs Y, Hughes TJ. Isogeometric analysis of the Cahn-Hilliard phase-field model. Computer methods in applied mechanics and engineering. $2008 \mathrm{Sep} 15$;197(49-50):4333-52.

[4] Chan PK, Rey AD. A numerical method for the nonlinear Cahn-Hilliard equation with nonperiodic boundary conditions. Computational materials science. 1995 Jan 1;3(3):377-92.

[5] Davis TA. Direct methods for sparse linear systems. Siam; 2006 Sep 1.

[6] Novick-Cohen A, Segel LA. Nonlinear aspects of the Cahn-Hilliard equation. Physica D: Nonlinear Phenomena. 1984 Mar 1;10(3):277-98.

[7] Cook HE. Brownian motion in spinodal decomposition. Acta metallurgica. 1970 Mar 1;18(3):297306.

[8] Castro AJ, inventor; Akzona Inc, assignee. Methods for making microporous products. United States patent US 4,247,498. 1981 Jan 27.

[9] Graham PD, Pervan AJ, McHugh AJ. The dynamics of thermal-induced phase separation in PMMA solutions. Macromolecules. 1997 Mar 24;30(6):1651-5.

[10] Barton BF, Graham PD, McHugh AJ. Dynamics of spinodal decomposition in polymer solutions near a glass transition. Macromolecules. 1998 Mar 10;31(5):1672-9.

[11] West JL. Phase separation of liquid crystals in polymers. Molecular Crystals and Liquid Crystals Incorporating Nonlinear Optics. 1988 Apr 1;157(1):427-41.

[12] Kim JY, Palffy-Muhoray P. Phase separation kinetics of a liquid crystal-polymer mixture. Molecular Crystals and Liquid Crystals. $1991 \mathrm{Jul}$ 1;203(1):93-100.

[13] Liu K, Kiran E. Kinetics of pressure-induced phase separation (PIPS) in solutions of polydimethylsiloxane in supercritical carbon dioxide: crossover from nucleation and growth to 
spinodal decomposition mechanism. The Journal of supercritical fluids, 1999 Sep 15;16(1):59-79..

[14] Ohnaga T, Inoue T. Growth and decay of concentration fluctuations in polymer-polymer mixtures. Journal of Polymer Science Part B: Polymer Physics. 1989 Jul;27(8):1675-89.

[15] Tran TL, Chan PK, Rousseau D. Morphology control in symmetric polymer blends using spinodal decomposition. Chemical engineering science. 2005 Dec 1;60(24):7153-9.

[16] Kwak KD, Okada M, Chiba T, Nose T. Growth rate of microdomains during phase separation by a two-step temperature jump. Macromolecules. 1993 Jul;26(15):4047-9.

[17] Tanaka H. Pattern formation caused by double quenches in binary polymer mixtures: Response of phase-separated structure to a second quench within a two-phase region. Physical Review E. 1993 Apr 1;47(4):2946.

[18] Tran-Cong-Miyata Q, Nishigami S, Ito T, Komatsu S, Norisuye T. Controlling the morphology of polymer blends using periodic irradiation. Nature materials. $2004 \mathrm{Jul} ; 3(7): 448$.

[19] Harada A, Tran-Cong Q. Modulated phases observed in reacting polymer mixtures with competing interactions. Macromolecules. 1997 Mar 24;30(6):1643-50.

[20] Furukawa H. Pattern formations in unstable network: model for cross-linked polymer chains. Journal of the Physical Society of Japan. 1994 Nov;63(11):3919-22.

[21] Okada M, Masunaga H, Furukawa H. Concentric pattern formation during phase separation induced by a cross-linking reaction. Macromolecules. 2000 Oct 3;33(20):7238-40.

[22] Kyu T, Lee JH. Nucleation initiated spinodal decomposition in a polymerizing system. Physical review letters. 1996 May 13;76(20):3746.

[23] Hirose A, Shimada K, Hayashi C, Nakanishi H, Norisuye T, Tran-Cong-Miyata Q. Polymer networks with bicontinuous gradient morphologies resulting from the competition between phase separation and photopolymerization. Soft matter. 2016;12(6):1820-9.

[24] Enge W, Köhler W. Thermal diffusion in a critical polymer blend. Physical Chemistry Chemical Physics. 2004;6(9):2373-8.

[25] Kumaki J, Hashimoto T, Granick S. Temperature gradients induce phase separation in a miscible polymer solution. Physical review letters. 1996 Sep 2;77(10):1990.

[26] Furukawa H. Phase separation by directional quenching and morphological transition. Physica A: Statistical Mechanics and its Applications. 1992 Jan 1;180(1-2):128-55.

[27] Okinaka J, Tran-Cong Q. Directional phase separation of a binary polymer mixture driven by a temperature gradient. Physica D: Nonlinear Phenomena. 1995 Jun 15;84(1-2):23-30.

[28] Tran-Cong Q, Okinaka J. Polymer blends with spatially graded structures prepared by phase separation under a temperature gradient. Polymer Engineering \& Science. 1999 Feb;39(2):365-74. 
[29] Kukadiya SB, Chan PK, Mehrvar M. The Ludwig-Soret Effect on the Thermally Induced Phase Separation Process in Polymer Solutions: A Computational Study. Macromolecular Theory and Simulations. 2009 Feb 25;18(2):97-107.

[30] Anders D, Weinberg K. Thermophoresis in binary blends. Mechanics of Materials. 2012 Apr 1;47:3350.

[31] Voit A, Krekhov A, Enge W, Kramer L, Köhler W. Thermal patterning of a critical polymer blend. Physical review letters. 2005 Jun 2;94(21):214501.

[32] Lee KW, Chan PK, Feng X. Morphology development and characterization of the phase-separated structure resulting from the thermal-induced phase separation phenomenon in polymer solutions under a temperature gradient. Chemical Engineering Science, 2004 Apr 1;59(7):1491-504.

[33] Puri S, Binder K. Surface-directed spinodal decomposition: phenomenology and numerical results. Physical Review A. 1992 Oct 1;46(8):R4487.

[34] Puri S, Frisch HL. Surface-directed spinodal decomposition: modelling and numerical simulations. Journal of Physics: Condensed Matter. 1997 Mar 10;9(10):2109.

[35] Yan LT, Xie XM. Lamellar morphology induced by two-step surface-directed spinodal decomposition in binary polymer mixture films. The Journal of chemical physics. 2008 Jan 21;128(3):034901.

[36] Yan LT, Li J, Xie XM. Three-dimensional numerical simulations of lamellar structure via two-step surface-directed phase separation in polymer blend films. The Journal of chemical physics. $2008 \mathrm{Jun}$ 14;128(22):224906.

[37] Li JL, Yan LT, Xie XM. Phase dynamics and wetting layer formation mechanisms of pattern-directed phase separation in binary polymer mixture films with asymmetry compositions. Polymer. $2009 \mathrm{Apr}$ 24;50(9):2172-80.

[38] Yan LT, Xie XM. Wetting-layer formation mechanisms of surface-directed phase separation under different quench depths with off-critical compositions in polymer binary mixture. The Journal of chemical physics. 2007 Feb 14;126(6):064908.

[39] Shang Y, Kazmer D, Wei M, Mead J, Barry C. Numerical simulation of phase separation of immiscible polymer blends on a heterogeneously functionalized substrate. The Journal of chemical physics. 2008 Jun 14;128(22):224909.

[40] Lee BP, Douglas JF, Glotzer SC. Filler-induced composition waves in phase-separating polymer blends. Physical Review E. 1999 Nov 1;60(5):5812.

[41] Karim A, Douglas JF, Nisato G, Liu DW, Amis EJ. Transient target patterns in phase separating filled polymer blends. Macromolecules. 1999 Sep 7;32(18):5917-24.

[42] Tabatabaieyazdi M, Chan PK, Wu J. A computational study of short-range surface-directed phase separation in polymer blends under a linear temperature gradient. Chemical Engineering Science. 
2015 Dec 1;137:884-95.

[43] Jayalakshmi Y, Khalil B, Beysens D. Phase separation under a weak concentration gradient. Physical review letters. 1992 Nov 23;69(21):3088.

[44] Lacasta AM, Sancho JM, Yeung C. Phase separation dynamics in a concentration gradient. EPL (Europhysics Letters). 1994 Aug 1;27(4):291.

[45] Chan PK. Effect of concentration gradient on the thermal-induced phase separation phenomenon in polymer solutions. Modelling and Simulation in Materials Science and Engineering. 2006 Jan 5;14(1):41.

[46] Matsuyama H, Berghmans S, Batarseh MT, Lloyd DR. Effects of thermal history on anisotropic and asymmetric membranes formed by thermally induced phase separation. Journal of membrane science. 1998 Feb 2;142(1):27-42.

[47] He D, Ulbricht $M$. Surface-selective photo-grafting on porous polymer membranes via a synergist immobilization method. Journal of Materials Chemistry. 2006;16(19):1860-8.

[48] Yu G, Zou X, Sun L, Liu B, Wang Z, Zhang P, Zhu G. Constructing Connected Paths between UiO-66 and PIM-1 to Improve Membrane CO2 Separation with Crystal-Like Gas Selectivity. Advanced Materials. 2019 Feb 25:1806853.

[49] Barton BF, McHugh AJ. Modeling the dynamics of membrane structure formation in quenched polymer solutions. Journal of Membrane Science. 2000 Feb 14;166(1):119-25.

[50] Matsuyama H, Yuasa M, Kitamura Y, Teramoto M, Lloyd DR. Structure control of anisotropic and asymmetric polypropylene membrane prepared by thermally induced phase separation. Journal of membrane science. 2000 Nov 15;179(1-2):91-100.

[51] Pulko I, Wall J, Krajnc P, Cameron NR. Ultra-High Surface Area Functional Porous Polymers by Emulsion Templating and Hypercrosslinking: Efficient Nucleophilic Catalyst Supports. Chemistry-A European Journal. 2010 Feb 22;16(8):2350-4.

[52] Detoni C, Gierlich CH, Rose M, Palkovits R. Selective liquid phase adsorption of 5hydroxymethylfurfural on nanoporous hyper-cross-linked polymers. ACS Sustainable Chemistry \& Engineering. 2014 Aug 25;2(10):2407-15.

[53] Dalmoro A, Barba AA, Lamberti G, Grassi M, d'Amore M. Pharmaceutical applications of biocompatible polymer blends containing sodium alginate. Advances in Polymer Technology. 2012 Sep;31(3):219-30.

[54] Evans DF, Wennerström H. The colloidal domain: where physics, chemistry, biology, and technology meet.

[55] Nyholm L, Nyström G, Mihranyan A, Strømme M. Toward flexible polymer and paper-based energy storage devices. Advanced Materials. 2011 Sep 1;23(33):3751-69. 
[56] Yamaguchi T, Miyata F, Nakao SI. Polymer Electrolyte Membranes with a Pore-Filling Structure for a Direct Methanol Fuel Cell. Advanced Materials. 2003 Jul 17;15(14):1198-201.

[57] Bahadur B, editor. Liquid crystals: applications and uses. World scientific; 1990.

[58] Higgins DA. Probing the mesoscopic chemical and physical properties of polymer-dispersed liquid crystals. Advanced Materials. 2000 Feb;12(4):251-64.

[59] Norde W. Colloids and interfaces in life sciences. CRC Press; 2003 Jun 20.

[60] Gray JJ. The interaction of proteins with solid surfaces. Current opinion in structural biology. 2004 Feb 1;14(1):110-5.

[61] Yaseen M, Salacinski HJ, Seifalian AM, Lu JR. Dynamic protein adsorption at the polyurethane copolymer/water interface. Biomedical Materials. 2008 Sep 3;3(3):034123.

[62] Mishra K, Hashmi SA, Rai DK. Nanocomposite blend gel polymer electrolyte for proton battery application. Journal of Solid State Electrochemistry. 2013 Mar 1;17(3):785-93.

[63] Ramanathan T, Abdala AA, Stankovich S, Dikin DA, Herrera-Alonso M, Piner RD, Adamson DH, Schniepp HC, Chen XR, Ruoff RS, Nguyen ST. Functionalized graphene sheets for polymer nanocomposites. Nature nanotechnology. 2008 Jun;3(6):327.

[64] Kimmins SD, Cameron NR. Functional porous polymers by emulsion templating: recent advances. Advanced Functional Materials. 2011 Jan 21;21(2):211-25.

[65] Guy D, Lestriez B, Guyomard D. New composite electrode architecture and improved battery performance from the smart use of polymers and their properties. Advanced Materials. 2004 Mar 18;16(6):553-7.

[66] Mukherjee D, Gowda Y. K G, Makri Nimbegondi Kotresh H, Sampath S. Porous, hyper-cross-linked, three-dimensional polymer as stable, high rate capability electrode for lithium-ion battery. ACS applied materials \& interfaces. 2016 Nov 22;9(23):19446-54.

[67] Tran-Cong Q, Nakano H, Okinaka J, Kawakubo R. Miscibility of poly (2-chlorostyrene) and poly (vinyl methyl ether) blends. Polymer. 1994 Mar 1;35(6):1242-7.

[68] Elliott CM. The Cahn-Hilliard model for the kinetics of phase separation. InMathematical models for phase change problems 1989 (pp. 35-73). Birkhäuser Basel.

[69] De Gennes PG, Gennes PG. Scaling concepts in polymer physics. Cornell university press; 1979.

[70] Schmidt I, Binder K. Model calculations for wetting transitions in polymer mixtures. Journal de Physique. 1985 Oct 1;46(10):1631-44.

[71] Flory PJ. Thermodynamics of high polymer solutions. The Journal of chemical physics. 1942 Jan;10(1):51-61. 
[72] Bates PW, Xun JP. Metastable patterns for the Cahn-Hilliard equation, part I. Journal of differential equations. $1994 \mathrm{Jul}$ 15;111(2):421-57.

[73] Bates PW, Xun JP. Metastable patterns for the Cahn-Hilliard equation: Part II. Layer dynamics and slow invariant manifold. Journal of differential equations. 1995 Mar 20;117(1):165-216.

[74] Bates PW, Fife PC. The dynamics of nucleation for the Cahn-Hilliard equation. SIAM Journal on Applied Mathematics. 1993 Aug;53(4):990-1008.

[75] Tran-Cong Q, Harada A. Reaction-induced ordering phenomena in binary polymer mixtures. Physical review letters. $1996 \mathrm{Feb}$ 12;76(7):1162.

[76] Tamai T, Imagawa A, Tran-Cong Q. Semi-interpenetrating polymer networks prepared by in situ photo-crosslinking of miscible polymer blends. Macromolecules. 1994 Dec;27(25):7486-9.

[77] Jiang BT, Chan PK. Effect of concentration gradient on the morphology development in polymer solutions undergoing thermally induced phase separation. Macromolecular Theory and Simulations. 2007 Sep 25;16(7):690-702.

[78] Lapidus L, Pinder GF. Numerical solution of partial differential equations in science and engineering. John Wiley \& Sons; 2011 Feb 14.

[79] Chan PK. Formation and Performance of Polymer Dispersed Liquid Crystal Films. McGill University; 1997 May.

[80] Ermi BD, Karim A, Douglas JF. Formation and dissolution of phase-separated structures in ultrathin blend films. Journal of Polymer Science Part B: Polymer Physics. 1998 Jan 15;36(1):191-200.

[81] Huston EL, Cahn JW, Hilliard JE. Spinodal decomposition during continuous cooling. Acta metallurgica. 1966 Sep 1;14(9):1053-62.

[82] Tanaka H, Lovinger AJ, Davis DD. Pattern evolution caused by dynamic coupling between wetting and phase separation in binary liquid mixture containing glass particles. Physical review letters. 1994 Apr 18;72(16):2581.

[83] Tran-Cong Q, Kawai J, Endoh K. Modes selection in polymer mixtures undergoing phase separation by photochemical reactions. Chaos: An Interdisciplinary Journal of Nonlinear Science. 1999 Jun;9(2):298-307.

[84] Tran-Cong-Miyata $Q$, Nakanishi H. Phase separation of polymer mixtures driven by photochemical reactions: current status and perspectives. Polymer International. 2017 Feb;66(2):213-22.

[85] Yu G, Zou X, Sun L, Liu B, Wang Z, Zhang P, Zhu G. Constructing Connected Paths between UiO-66 and PIM-1 to Improve Membrane CO2 Separation with Crystal-Like Gas Selectivity. Advanced Materials. 2019 Feb 25:1806853. 


\section{Appendix A Complimentary theoretical}

\section{background and numerical methods}

In this chapter, some of the fundamental theories of dynamics in binary polymer mixtures as well as numerical methods are demonstrated. They may be of assistance in reading preceding chapters. Though the readers are encouraged to review previous publications and textbooks for most of the mathematical background, some of the simplest background is shown here for the ease of reading through this thesis and also for the sake of neatness in other chapters. Some research methods are also explained throughout this chapter.

\section{A.1 Solution to linearized Cahn-Hilliard and determining characteristic frequency in all dimensions}

In this section, the formula for obtaining characteristic frequency is derived. This formula essentially plays crucial role in this entire thesis. Each simulation is run strategically so that the most important structures are within appropriate frequency range for analysis. If the frequency is too large, some numerical errors might results because it is too close to or even exceeds Nyquist frequency, or the droplets might not be in reasonable shapes. 2-d mesh for this thesis is $256 \times 256$, but $3-d$ mesh is only $32 \times 32 \times 32$, thus one must pay close attention so the characteristic frequency of the deepest quench is not too high. On the other hand, if the characteristic frequency is too low, a large macro-structures that are too few in number may result. Such results might make the observations statistically less valuable for a number of analyses, such as droplets count. 
The characteristic frequency is obtained with linearized Cahn-Hilliard equation, which is appropriate for phase separation in early stage and can be solved easily with undergraduate level mathematics.

Let us consider 2-d case. Suppose the extremum of growth of structure occurs at a specific frequency

$$
k_{1}=k_{1}^{\prime} \text { and } k_{2}=k_{2}^{\prime}
$$

In 2-d (xy-coordinates):

$$
\begin{gathered}
\nabla^{2} c=\frac{\partial^{2} c}{\partial x^{2}}+\frac{\partial^{2} c}{\partial y^{2}} \\
\nabla^{4} c=\frac{\partial^{4} c}{\partial x^{4}}+2 \frac{\partial^{4} c}{\partial x^{2} \partial y^{2}}+\frac{\partial^{4} c}{\partial y^{4}}
\end{gathered}
$$

Recall linearized Cahn-Hilliard equation:

$$
\frac{\partial c}{\partial t}=\left.D T \frac{\partial^{2} f}{\partial c^{2}}\right|_{c=c_{a v e}} \nabla^{2} c-\nabla^{4} c
$$

Then, linearized Cahn-Hilliard in 2-d equation can be written as:

$$
\frac{\partial c}{\partial t}=\left.D T \frac{\partial^{2} f}{\partial c^{2}}\right|_{c=c_{\text {ave }}}\left(\frac{\partial^{2} c}{\partial x^{2}}+\frac{\partial^{2} c}{\partial y^{2}}\right)-\left(\frac{\partial^{4} c}{\partial x^{4}}+2 \frac{\partial^{4} c}{\partial x^{2} \partial y^{2}}+\frac{\partial^{4} c}{\partial y^{4}}\right)
$$

In order to Fourier transform this equation, $x$-component is Fourier transformed. Define:

$$
\mathcal{F}_{x}[c]=\hat{c}\left(k_{1}\right)=\int_{-\infty}^{\infty} c e^{-2 \pi k_{1} x} \partial x
$$


Thus, one obtains the equation with only one component transformed

$$
\frac{\partial \hat{c}}{\partial t}=\left.\mathrm{D} T \frac{\partial^{2} f}{\partial c^{2}}\right|_{c=c_{\text {ave }}}\left[\left(2 \pi k_{1} i\right)^{2} \hat{c}+\frac{\partial^{2} \hat{c}}{\partial y^{2}}\right]-\left[\left(2 \pi k_{1} i\right)^{4} \hat{c}+2\left(2 \pi k_{1} i\right)^{2} \frac{\partial^{2} \hat{c}}{\partial y^{2}}+\frac{\partial^{4} \hat{c}}{\partial y^{4}}\right]
$$

To further simplify complex number:

$$
\frac{\partial \hat{c}}{\partial t}=\left.\mathrm{D} T \frac{\partial^{2} f}{\partial c^{2}}\right|_{c=c_{a v e}}\left[-\left(2 \pi k_{1}\right)^{2} \hat{c}+\frac{\partial^{2} \hat{c}}{\partial y^{2}}\right]-\left[\left(2 \pi k_{1}\right)^{4} \hat{c}-2\left(2 \pi k_{1}\right)^{2} \frac{\partial^{2} \hat{c}}{\partial y^{2}}+\frac{\partial^{4} \hat{c}}{\partial y^{4}}\right]
$$

Next, y-component is also transformed:

$$
\mathcal{F}_{y}[\hat{c}]=\hat{\hat{c}}\left(k_{1}, k_{2}\right)=\int_{-\infty}^{\infty} \hat{c}\left(k_{1}\right) e^{-2 \pi k_{2} y} \partial y
$$

To complete Fourier transform:

$$
\begin{aligned}
\frac{\partial \hat{\hat{c}}}{\partial t}=\left.\mathrm{D} T \frac{\partial^{2} f}{\partial c^{2}}\right|_{c=c_{\text {ave }}}\left[-\left(2 \pi k_{1}\right)^{2} \hat{\hat{c}}\right. \\
\\
\left.+\left(2 \pi k_{2} i\right)^{2} \hat{\hat{c}}\right]-\left[\left(2 \pi k_{1}\right)^{4} \hat{\hat{c}}-2\left(2 \pi k_{1}\right)^{2}\left(2 \pi k_{2} i\right)^{2} \hat{\hat{c}}+\left(2 \pi k_{2} i\right)^{4} \hat{\hat{c}}\right]
\end{aligned}
$$

By computing complex number:

$$
\begin{aligned}
\frac{\partial \hat{\hat{c}}}{\partial t}=-\left.\mathrm{D} T \frac{\partial^{2} f}{\partial c^{2}}\right|_{c=c_{\text {ave }}}\left[\left(2 \pi k_{1}\right)^{2} \hat{\hat{c}}+\left(2 \pi k_{2}\right)^{2} \hat{\hat{c}}\right]-\left[\left(2 \pi k_{1}\right)^{4} \hat{\hat{c}}\right. \\
\left.+2\left(2 \pi k_{1}\right)^{2}\left(2 \pi k_{2}\right)^{2} \hat{\hat{c}}+\left(2 \pi k_{2}\right)^{4} \hat{\hat{c}}\right]
\end{aligned}
$$

To further re-arrange and simplify:

$$
\frac{\partial \hat{c}}{\partial t}+\left\{\left.\mathrm{D} T \frac{\partial^{2} f}{\partial c^{2}}\right|_{c=c_{\text {ave }}}\left[\left(2 \pi k_{1}\right)^{2}+\left(2 \pi k_{2}\right)^{2}\right]+\left[\left(2 \pi k_{1}\right)^{2}+\left(2 \pi k_{2}\right)^{2}\right]^{2}\right\} \hat{\hat{c}}=0
$$


This is a linear and first order ordinary differential equation. Solving it is straightforward with initial condition also transformed as following:

$$
\hat{\hat{c}}\left(k_{1}, k_{2}, t=0\right)=\hat{\hat{c}}_{0}
$$

Note that boundary conditions are not accounted in solving using Fourier transform. Thus, the results are normally best manifested far away from the boundaries. Indeed, throughout this entire thesis, structures at the boundaries usually have characteristic frequency that is higher than predicted.

Now, one arrives at analytical solution by solving simply ODE:

$$
\hat{\hat{c}}=\hat{\hat{c}}_{0} e^{-t\left\{\left.\mathrm{D} T \frac{\partial^{2} f}{\partial c^{2}}\right|_{c=c_{O}}\left[\left(2 \pi k_{1}\right)^{2}+\left(2 \pi k_{2}\right)^{2}\right]+\left[\left(2 \pi k_{1}\right)^{2}+\left(2 \pi k_{2}\right)^{2}\right]^{2}\right\}}
$$

This expression shows that the rate of growth at specific frequency is dictated by power term of analytical solution above. It is often defined as a growth factor:

$$
R\left(k_{1}, k_{2}\right)=-t\left\{\left.\mathrm{D} T \frac{\partial^{2} f}{\partial c^{2}}\right|_{c=c_{o}}\left[\left(2 \pi k_{1}\right)^{2}+\left(2 \pi k_{2}\right)^{2}\right]+\left[\left(2 \pi k_{1}\right)^{2}+\left(2 \pi k_{2}\right)^{2}\right]^{2}\right\}
$$

In an attempt to determine the extremum, or the slowest or fastest growth rate, set:

$$
\left.\nabla^{k} R\right|_{k_{1}=k_{1}^{\prime}, k_{2}=k_{2}^{\prime}}=\overrightarrow{0}
$$

Where:

$$
\nabla^{k}=\frac{\partial}{\partial k_{1}} \hat{k}_{1}+\frac{\partial}{\partial k_{2}} \hat{k}_{2}
$$

In other words: 


$$
\left.\frac{\partial R}{\partial k_{1}}\right|_{k_{1}=k_{1}^{\prime}, k_{2}=k_{2}^{\prime}}=\left.\frac{\partial R}{\partial k_{2}}\right|_{k_{1}=k_{1}^{\prime}, k_{2}=k_{2}^{\prime}}=0
$$

Given equations above, solving this is a straightforward process and one can easily obtain:

$$
k_{1}^{\prime 2}+k_{2}^{\prime 2}=\left(\frac{1}{2 \pi} \sqrt{\left.\frac{-1}{2} \mathrm{D} T \frac{\partial^{2} f}{\partial c^{2}}\right|_{c=c_{o}}}\right)^{2}
$$

This is an equation of the circle with right-hand-side as radius. Thus, in the early stage of single quench from uniformly mixed state, where linear Cahn-Hilliard equation is generally valid, one expects to see a ring-structure on the amplitude plot of Fourier-transform with the radius as computed from the right-hand-side. This equation will play extremely important role throughout this thesis as each simulation is strategically carried out within appropriate range.

Note that, the radicant of right-hand-side is real only when $\left.\frac{\partial^{2} f}{\partial c^{2}}\right|_{c=c_{o}} \leq 0$, or only when the solution is in unstable region. When the solution is in metastable or stable region, growth factor is negative at all frequencies. Furthermore, the growth factor is larger negative when absolute values of $k_{1}$ and $k_{2}$ are larger. Thus, high frequency noises get eliminated quickly first, while low frequency structure persists the longest. Nonetheless, the solution will constantly go back to uniformly mixed state unless there is appropriate external influence such as noise.

By following similar procedure as above, one can further obtain equations to obtain characteristic frequency for 1-d and 3-d:

For 1-d:

$$
k^{\prime 2}=\left(\frac{1}{2 \pi} \sqrt{\left.\frac{-1}{2} \mathrm{D} T \frac{\partial^{2} f}{\partial c^{2}}\right|_{c=c_{o}}}\right)^{2}
$$

For 3-d: 


$$
k_{1}^{\prime 2}+k_{2}^{\prime 2}+k_{3}^{\prime 2}=\left(\frac{1}{2 \pi} \sqrt{\left.\frac{-1}{2} \mathrm{D} T \frac{\partial^{2} f}{\partial c^{2}}\right|_{c=c_{o}}}\right)^{2}
$$

Obviously, the equation for 3-d is the surface of a sphere with radius as shown on right-handside. The radius is ultimately the characteristic frequency, i.e.

$$
f_{c}=\frac{1}{2 \pi} \sqrt{\left.\frac{-1}{2} \mathrm{D} T \frac{\partial^{2} f}{\partial c^{2}}\right|_{c=c_{o}}}
$$

\section{A.2 Flory-Huggins equation}

Flory-Huggins theory [71] is commonly used to describe multi-phase polymer solutions. It is derived based on finite lattice model using statistics accompanied with some approximations and assumptions. Therefore, it has its own limitations as well. In this chapter, the derivations for free energy of binary polymer solution are shown. The theory can be extended to the solutions with even more phases.

In a binary polymer solution, the Gibbs free energy of mixing is the competing process of the solution in mixed state and the solution in the unmixed state. Therefore, it is expressed as following:

$$
\Delta G^{M}=G_{12}-\left(G_{1}+G_{2}\right)
$$

Where $G_{i}$ the Gibb's free energy, and the subscript $i$ indicates phases. $i=12$ means the mixed state. This way, if $\Delta G^{M}<0$, mixed state is more favorable. The opposite is true for $\Delta G^{M}>0$.

Next, finite element lattice model is defined: Suppose a domain contains $N_{0}$ cells of equal volume. In each cell, either the solvent molecule or a segment of polymer occupies. Each solvent molecule and polymer segment has the size of 1 unit cell. This way, the volume of the entire cell is: 


$$
V=V_{1} N_{0}=V_{1} N_{1}+V_{2} N_{2}
$$

Where $V_{i}$ is the total volume of component $i$. For this section, $i=1$ is the solvent and $i=2$ is the polymer. Let $r=V_{2} / V_{1}$, then $r$ means the number of cells a polymer occupies, or the chain length. One can also infer the relationship:

$$
N_{0}=N_{1}+r N_{2}
$$

Now, suppose one is to add polymer chains into the domain one by one. Every time before new polymer chain is added, the number of cells that are still vacant are the difference between the total number of cells and the total number of cells occupied by each segment of each polymer chain already in place:

$$
N_{0}-r i
$$

Where $i$ is the number of polymers that are already in place. This is utlimately the number of possible ways the first segment of a polymer chain is added as one desires to add polymer segment one by one. Next, the contiguous polymer segment is added adjacent to the first segment. First, one can recognize that, at this point, the probability that the adjacent cell is empty is, to a reasonable approximation for large volume of cell:

$$
\left(N_{0}-r i\right) / N_{0}
$$

Suppose $z$ is the number of cells adjacent to it. Then, the number of possible way the second segment is added is:

$$
z\left(N_{0}-r i\right) / N_{0}
$$


Now, from the third segment, since it is certain that one adjacent cell has already been filled by the first segment, one less cell is available. Thus, the number of ways third segment and so forth can be added is:

$$
(z-1)\left(N_{0}-r i\right) / N_{0}
$$

This process is repeated until the entire polymer chain has been added. Thus, the number of ways a polymer chain is added is the multiplications of possibilities of each segment shown above. After simplification, it is:

$$
\omega_{i+1}=\left(N_{0}-r i\right)^{r}\left[(z-1) / N_{o}\right]^{r-1}
$$

This way, the number of ways all polymers can be added into a cell is:

$$
\prod_{i=1}^{N_{2}} \omega_{i}
$$

After all polymers have been added, the remaining vacant cells are filled with solvent molecules. Thus, this is also the total number of possible overall configurations. Assume that all polymers are identical or indistinguishable, then the total number of ways all polymers set into the domain is, by combinatorics and simplification:

$$
\Omega_{p}=\frac{1}{N_{2} !} \prod_{i=1}^{N_{2}} \omega_{i}=\frac{1}{N_{2} !}\left(\frac{Z-1}{N_{0}}\right)^{N_{2}(r-1)} r^{N_{2} r} \prod_{i=1}^{N_{2}}\left(\frac{N_{0}}{r}-i+1\right)^{r}
$$

The product term,

$$
\left(\frac{N_{0}}{r}+1-1\right)^{r}\left(\frac{N_{0}}{r}+1-2\right)^{r}\left(\frac{N_{0}}{r}+1-3\right)^{r} \ldots\left(\frac{N_{0}}{r}+1-N_{2}\right)^{r}
$$

Is equivalent to 


$$
\left[\frac{\left(\frac{N_{o}}{r}\right) !}{\left(\frac{N_{o}}{r}-N_{2}\right) !}\right]^{r}=\left[\frac{\left(\frac{N_{o}}{r}\right) !}{\left(\frac{N_{1}}{r}\right) !}\right]^{r}
$$

Thus, a simpler version to work with now becomes:

$$
\Omega_{p}=\frac{r^{N_{2} r}}{N_{2} !}\left(\frac{Z-1}{N_{0}}\right)^{N_{2}(r-1)}\left[\frac{\left(\frac{N_{o}}{r}\right) !}{\left(\frac{N_{1}}{r}\right) !}\right]^{r}
$$

By Boltzmann equation completes the equation for the entropy of mixing:

$$
S^{M}=k_{b} \ln \Omega_{p}
$$

Where $k_{b}$ is Boltzmann constant. After substitution of $\Omega_{p}$ and some approximations, which will not be shown here, and further modifications, the entropy of mixing is:

$$
S^{M}=k_{b}\left\{-N_{1} \ln \frac{N_{1}}{N_{o}}-N_{2} \ln \frac{N_{2}}{N_{o}}+N_{2}(r-1) \ln \frac{z-1}{e}\right\}
$$

To convert this into a more useful form, $N_{2} \ln r$ is added and subtracted, simplifying the equation to:

$$
S^{M}=k_{b}\left\{-N_{1} \ln \frac{N_{1}}{N_{o}}-N_{2} \ln \frac{r N_{2}}{N_{o}}+N_{2}\left[(r-1) \ln \frac{z-1}{e}+\ln r\right]\right\}
$$

The entropy of pure solvent can be determined by setting $N_{1}=N_{o}$ and $N_{2}=0$, which yields $S_{1}=0$. Similarly, the entropy of pure polymer is determined by $N_{1}=0$ and $N_{2}=N_{o}$, which yields:

$$
S_{2}=N_{2}\left[(r-1) \ln \frac{z-1}{e}+\ln r\right]
$$


The change in the entropy of mixing is the competition of the entropy of mixed state and that of demixed state, so:

$$
\Delta S^{M}=S^{M}-\left(S_{1}+S_{2}\right)=-k_{b}\left(N_{1} \ln \frac{N_{1}}{N_{o}}+N_{2} \ln \frac{r N_{2}}{N_{o}}\right)
$$

At this point, the volumetric fraction of the solvent and polymer are defined as $c_{1}$ and $c_{2}$, respectively:

$$
\begin{gathered}
c_{1}=\frac{V_{1} N_{1}}{V}=\frac{N_{1}}{N_{o}} \\
c_{2}=1-c_{1}=\frac{V_{2} N_{2}}{V}=\frac{r N_{2}}{N_{o}}
\end{gathered}
$$

$n_{1}$ and $n_{2}$ are defined here as the chain lengths of solvent and polymer, respectively. Furthermore, $v$ is defined as the volume of one cell. This way, $V_{1}=n_{1} v$ and $V_{2}=n_{2} v$. The change in the entropy of mixing has the following final form which is used for this thesis:

$$
\Delta S^{M}=-\frac{k_{b} V}{v}\left(\frac{c_{1}}{n_{1}} \ln c_{1}+\frac{c_{2}}{n_{2}} \ln c_{2}\right)
$$

The change of enthalpy of mixing can be thought of as the total energy generated by first breaking the intermolecular interactions of same entities then create another ones between two different entities. Thus, for each new intermolecular interaction of two different species, the energy in the energy is:

$$
\Delta \varepsilon_{12}=\varepsilon_{12}-\frac{1}{2}\left(\varepsilon_{11}+\varepsilon_{22}\right)
$$

Where $\varepsilon_{12}, \varepsilon_{11}$, and $\varepsilon_{22}$ are the energy of intermolecular interaction between two different species, that of the same species of component 1, and that of the same species between 
component 2, respectively. The enthalpy of mixing is the summation of all process described above, thus the number of newly formed interactions between different species. Recall that each segment of polymer has $z$ adjacent cells, and there are $r$ segments. The chance of the adjacent cell containing solvent molecule is really the concentration of solvent, $c_{1}$. Thus, each polymer is surrounded by $c_{1} r z$ solvent molecules. Since there are $N_{2}$ polymers, the enthalpy of mixing can be derived as van Laar expression:

$$
\Delta H^{M}=N_{2} c_{1} r z \Delta \varepsilon_{12}=N_{1} c_{2} z \Delta \varepsilon_{12}=\frac{V c_{1} c_{2} z \Delta \varepsilon_{12}}{v n_{1}}
$$

Often, the study of multiphase polymer solution is done by introducing dimensionless parameter and relating intermolecular attraction to it, Boltzmann constant and temperature:

$$
k_{b} T \chi=z \Delta \varepsilon_{12}
$$

Now, the final form of enthalpy of mixing for this thesis is:

$$
\Delta H^{M}=\frac{k_{b} T \chi c_{1} c_{2}}{v n_{1}}
$$

The dimensionless parameter, $\chi$, is still under the subject of study. It is often measured experimentally by curve fitting. Often, for upper critical temperature solution, it is related to the temperature by the following form:

$$
\chi=a+\frac{b}{T} \text { where } a, b \in \mathbb{R}
$$

Sometimes, it shows some non-linearity as following:

$$
\chi=a+\frac{b}{T}+\frac{c}{T^{2}} \text { where } a, b, c \in \mathbb{R}
$$

Now, it is ready to derive Gibb's free energy of mixing for local energy of solution: 


$$
\begin{gathered}
\Delta G^{M}=\Delta H^{M}-T \Delta S^{M} \\
\Delta G^{M}=\frac{k_{b} T V}{v}\left(\frac{c_{1}}{n_{1}} \ln c_{1}+\frac{c_{2}}{n_{2}} \ln c_{2}+\frac{c_{1} c_{2}}{n_{1}} \chi\right)
\end{gathered}
$$

This is to assume that $c_{1}$ and $c_{2}$ are the same throughout entire domain. The free energy of solution with concentration fluctuation is determined by volume integral of local free energy. Given this reasoning, one may write local free energy in a more convenient form which has concentration of solvent $c$ as parameter:

$$
f=\frac{k_{b} T}{v}\left(\frac{c}{n_{1}} \ln c+\frac{1-c}{n_{2}} \ln (1-c)+\frac{c(1-c)}{n_{1}} \chi\right)
$$

This completes the derivation for Flory-Huggins equation.

\section{A.3 Theory of Cahn-Hilliard}

In an attempt to express free energy of a binary polymer solution, Cahn and Hilliard [1] attributed local free energy density of the solution to the contributions from local concentration and its environments, namely derivatives. They assumed that concentration gradient is small as compared to reciprocal of the intermolecular distance. This way, concentration and its derivatives can be treated as independent. Thus, local free energy of a system can be expressed as Taylor expansion of its derivatives, evaluated at about average concentration: 


$$
\begin{aligned}
f(\vec{c})=f\left(c, c_{x}, c_{y}, c_{z}, c_{x x}, c_{y y}, c_{z z}, c_{x y}, c_{x z}, \ldots\right) \\
=\left.f\right|_{\vec{c}=\vec{c}_{o}}+\left.\sum_{i}\left(\partial_{i} f\right)\right|_{\vec{c}=\vec{c}_{o}}\left(\partial_{i} c-\partial_{i} c_{o}\right) \\
+\left.\frac{1}{2} \sum_{i j}\left(\partial_{i j} f\right)\right|_{\vec{c}=\vec{c}_{o}}\left(\partial_{i} c-\partial_{i} c_{o}\right)\left(\partial_{j} c-\partial_{j} c_{o}\right) \\
+\left.\frac{1}{3} \sum_{i j k}\left(\partial_{i j k} f\right)\right|_{\vec{c}=\vec{c}_{o}}\left(\partial_{i} c-\partial_{i} c_{o}\right)\left(\partial_{j} c-\partial_{j} c_{o}\right)\left(\partial_{k} c-\partial_{k} c_{o}\right) \\
+\cdots
\end{aligned}
$$

Where:

$$
\begin{gathered}
\vec{c}=\left[c, c_{x}, c_{y}, c_{z}, c_{x x}, c_{y y}, c_{z z}, c_{x y}, c_{x z}, \ldots\right] \\
\vec{c}_{o}=[c, 0,0,0, \ldots] \\
\partial_{i} c, \partial_{j} c, \partial_{k} c, \ldots=c_{x}, c_{y}, c_{z}, c_{x x}, c_{y y}, c_{z z}, c_{x y}, c_{x z}, \ldots \\
\partial_{i} f=\frac{\partial f}{\partial c_{x}}, \frac{\partial f}{\partial c_{y}}, \frac{\partial f}{\partial c_{z}}, \frac{\partial f}{\partial c_{x x}}, \ldots \\
\partial_{i j} f=\frac{\partial^{2} f}{\left(\partial c_{x}\right)^{2}}, \frac{\partial^{2} f}{\left(\partial c_{y}\right)^{2}}, \frac{\partial^{2} f}{\left(\partial c_{z}\right)^{2}}, \frac{\partial^{2} f}{\partial c_{x} \partial c_{y}}, \ldots \\
\partial_{i j k} f=\frac{\partial^{3} f}{\left(\partial c_{x}\right)^{3}}, \frac{\partial^{3} f}{\left(\partial c_{y}\right)^{3}}, \frac{\partial^{3} f}{\left(\partial c_{z}\right)^{3}}, \frac{\partial^{3} f}{\left(\partial c_{x}\right)^{2} \partial c_{y}}, \ldots
\end{gathered}
$$

For uniform solution, $\partial_{i} c_{0}=0$. By taking only leading terms, Taylor expansion can be reorganized as following:

$$
f=\left.f\right|_{\vec{c}=\vec{c}_{0}}+\sum_{i} L_{i} c_{i}+\sum_{i j} \kappa_{i j}^{(1)} c_{x_{i} x_{j}}+\frac{1}{2} \sum_{i j} \kappa_{i j}^{(2)} c_{x_{i}} c_{x_{j}}+\cdots
$$

Where:

$$
\begin{gathered}
c_{x_{i}}, c_{x_{j}}, \ldots=c_{x}, c_{y}, \ldots \\
c_{x_{i} x_{j}}=c_{x x}, c_{y y}, c_{z z}, c_{x y}, c_{x z}, \ldots
\end{gathered}
$$




$$
\begin{gathered}
L_{i}=\left.\frac{\partial f}{\partial c_{x_{i}}}\right|_{\vec{c}=\vec{c}_{0}} \\
\kappa_{i j}{ }^{(1)}=\left.\frac{\partial f}{\partial c_{x_{i} x_{j}}}\right|_{\vec{c}=\vec{c}_{0}} \\
\kappa_{i j}{ }^{(2)}=\left.\frac{\partial^{2} f}{\partial c_{x_{i}} \partial c_{x_{j}}}\right|_{\vec{c}=\vec{c}_{0}}
\end{gathered}
$$

$L_{i}$ is a polarization vector, which is expected to be zero in isotropic medium as there is no preferred direction of flow for the solution. Furthermore, since $\kappa_{i j}{ }^{(1)}$ and $\kappa_{i j}{ }^{(2)}$ are now assumed to be isotropic tensors, they are the multiples of Kronecker delta funcions, i.e.

$$
\kappa_{i j}^{(1)}=\kappa_{i j}^{(2)}=0 \text { if } i \neq j
$$

Now, the Taylor expansion only consists of a constant and terms with laplacian and gradient squared in the leading terms. Thus it simplifies to:

$$
f=\left.f\right|_{\vec{c}=\vec{c}_{0}}+\kappa_{1} \nabla^{2} c+\kappa_{2}|\nabla c|^{2}+\cdots
$$

Where:

$$
\begin{gathered}
\kappa_{1}=\left.\frac{\partial f}{\partial \nabla^{2} c}\right|_{\vec{c}=\vec{c}_{0}} \\
\kappa_{2}=\left.\frac{\partial^{2} f}{(\partial|\nabla c|)^{2}}\right|_{\vec{c}=\vec{c}_{0}}
\end{gathered}
$$

Thus, the free energy of the entire solution is:

$$
E=\int_{\Omega}\left(\left.f\right|_{\vec{c}=\vec{c}_{0}}+\kappa_{1} \nabla^{2} c+\kappa_{2}|\nabla c|^{2}+\cdots\right) \partial v
$$

Recall zero potential boundary condition: 


$$
\nabla c \cdot \hat{n}=0 \text { on } \partial \Omega
$$

Given this, the second term in the integral can be further modified:

$$
\begin{aligned}
\int_{\Omega}\left(\kappa_{1} \nabla^{2} c\right) \partial v & =\int_{\Omega} \nabla \cdot\left(\kappa_{1} \nabla c\right) \partial v-\int_{\Omega} \frac{\partial \kappa_{1}}{\partial c}|\nabla c|^{2} \partial v \\
& =\left.\int_{\Omega} \kappa_{1}(\nabla c \cdot \hat{n})\right|_{\partial \Omega} \partial v \\
& -\int_{\Omega} \frac{\partial \kappa_{1}}{\partial c}|\nabla c|^{2} \partial v=-\int_{\Omega} \frac{\partial \kappa_{1}}{\partial c}|\nabla c|^{2} \partial v
\end{aligned}
$$

Thus, the energy equation simplified to:

$$
E=\int_{\Omega}\left(\left.f\right|_{\vec{c}=\vec{c}_{0}}+\kappa|\nabla c|^{2}+\cdots\right) \partial v
$$

Where:

$$
\kappa=-\frac{\partial \kappa_{1}}{\partial c}+\kappa_{2}
$$

If the gradient contributes to energy term, i.e. higher gradient is less thermodynamically favorable, then $\kappa$ must be positive. This is the famous Cahn-Hilliard equation that inspired many researches in polymer mixtures. It is crucial that, upon research, one is able to derive this equation in order to understand some of the mechanisms and limitations from the assumptions, especially if one desires to study phase separation in case where the assumptions mentioned above are not valid, or when one desires to add more assumptions. For instance, it has been assumed in this thesis that $\kappa$ is a positive constant rather than dependent on composition. 


\section{A.4 Minimum energy of a binary mixture as pointed out by C.M. Elliott}

In this chapter, a configuration of a solution that yields optimal minimum energy is sought, i.e one seeks to obtain optimal concentration(s), $c_{e}$, if there is only 1 value, or $c_{e_{1}}, c_{e_{2}}, \ldots$, if there are multiple optimal concentrations within the solution. These concentrations are also called equilibrium concentration(s). Such configuration must satisfy:

$$
\min (E)=\min \int_{\Omega}\left[f(c)+\kappa|\nabla c|^{2}\right] \partial v
$$

Subject to boundary condition:

$$
\frac{1}{V} \int_{\Omega} c \partial v=c_{a v e}
$$

The second term is always zero or positive, thus the energy is minimum when:

$$
|\nabla c|^{2}=0 \forall \vec{x} \in \Omega
$$

Thus, problem reduces to only local free energy term:

$$
\min (E)=\min \left(E_{F H}\right)=\min \int_{\Omega} f \partial v
$$

Subject to boundary conditions:

$$
\int_{\Omega}\left(c-c_{0}\right)=0
$$

In order to optimize two equations above, Lagrange's multiplier is introduced: 


$$
E_{\alpha}=\int\left[f-\mu\left(c-c_{0}\right)\right] \partial v
$$

For some:

$$
\mu \in \mathbb{R}
$$

To minimize this integral, Euler-Lagrange operation is established:

$$
\frac{\partial E_{\alpha}}{\partial c}-\nabla \cdot \frac{\partial E_{\alpha}}{\partial(\nabla c)}+\cdots=\frac{\partial E_{\alpha}}{\partial c}=\frac{\partial f}{\partial c}-\mu=0
$$

By choosing appropriate value of $\mu, E_{\alpha}$ can be minimized. There are two assumptions here.

Hypothesis 1: There is only 1 equilibrium concentration.

Assume that:

$$
\mu=\left.\left(\frac{\partial f}{\partial c}\right)\right|_{c=c_{0}}
$$

This way, the following must be true for Euler-Lagrange equation to satisfy:

$$
c_{e}=c_{0}
$$

For this hypothesis, $c_{e}$ cannot be any other value.

Proof:

Assume:

$$
\mu=\left.\left(\frac{\partial f}{\partial c}\right)\right|_{c=c_{1}} \text { such that } c_{1} \neq c_{0}
$$

But: 


$$
\int_{\Omega}\left(c_{1}-c_{0}\right) \partial v \neq 0
$$

By this contradiction, proof is complete.

QED

Hypothesis 2: There exists two equilibrium values.

By noting that local free energy density has double well form, there exists

$$
\left.\left(\frac{\partial f}{\partial c}\right)\right|_{c=c_{a}}=\left.\left(\frac{\partial f}{\partial c}\right)\right|_{c=c_{b}}=\frac{\left.f\right|_{c=c_{b}}-\left.f\right|_{c=c_{a}}}{c_{b}-c_{a}}
$$

For some concentration:

$$
0 \leq c_{a} \& c_{b} \leq 1
$$

Graphically, this means that there exists a tangent line that passes through exactly two separate points on local free energy density curve. Indeed, this is true for all binary polymer blends in unstable and metastable region. It is also valid for Flory-Huggins theory. For this hypothesis, it is assumed that these two values are the equilibrium points:

$$
c_{e_{1}}=c_{a} \& c_{e_{2}}=c_{b}
$$

Thus:

$$
\mu=\left.\left(\frac{\partial f}{\partial c}\right)\right|_{c=c_{a}}=\left.\left(\frac{\partial f}{\partial c}\right)\right|_{c=c_{b}}
$$

This way, $\mu$ can always be the same value, regardless of whether $c=c_{a}$ or $c=c_{b}$ locally. 
This is a configuration in which, the concentration at any point must be either of the equilibrium values:

$$
c=\left\{\begin{array}{l}
c_{e_{1}} \forall \vec{x} \in \Omega_{e_{1}} \\
c_{e_{2}} \forall \vec{x} \in \Omega_{e_{2}}
\end{array}\right.
$$

Here, $\Omega_{e_{1}}$ and $\Omega_{e_{2}}$ are the domains in which the concentration is at $c_{e_{1}}$ and $c_{e_{2}}$, respectively. They make up the entire domain:

$$
\Omega=\Omega_{e_{1}} \cup \Omega_{e_{2}}
$$

By conservation of mass:

$$
\left.\int_{\Omega_{e_{1}}} c\right|_{e_{1}} \partial v+\left.\int_{\Omega_{e_{2}}} c\right|_{e_{2}} \partial v=\int_{\Omega} c_{0} \partial v
$$

This way, the following is true:

$$
\left.I_{E_{\alpha}}\right|_{c=c_{e_{1}}}=\left.I_{E_{\alpha}}\right|_{c=c_{e_{2}}}
$$

Where $I_{E_{\alpha}}$ is the integrand of $E_{\alpha}$. This can be explained easily graphically: Consider a tangent line touching $f$ at both $c=c_{a}$ and $c=\left.c_{b} \cdot I_{E_{\alpha}}\right|_{c=c_{e_{1}}}$ and $\left.I_{E_{\alpha}}\right|_{c=c_{e_{2}}}$ are represented as a point on this tangent line where $c=c_{o}$. This condition cannot be satisfied with any other value of $\mu$. Now, note that:

$$
\begin{aligned}
& \int_{\Omega_{e_{1}}}\left[\left.f\right|_{c=c_{e_{1}}}\right.\left.-\left.\frac{\partial f}{\partial c}\right|_{c=c_{e_{1}}}\left(c_{e_{1}}-c_{0}\right)\right] \partial v \\
&+\int_{\Omega_{e_{2}}}\left[\left.f\right|_{c=\Omega_{e_{2}}}-\left.\frac{\partial f}{\partial c}\right|_{c=\Omega_{e_{2}}}\left(c_{e_{2}}-c_{0}\right)\right] \partial v \\
&=\int_{\Omega}\left[\left.f\right|_{c=c_{e_{1}}}-\left.\frac{\partial f}{\partial c}\right|_{c=c_{e_{1}}}\left(c_{e_{1}}-c_{0}\right)\right] \partial v
\end{aligned}
$$




$$
<\left.\int_{\Omega} f\right|_{c=c_{o}} \partial v \forall c_{e_{1}}<c_{o}<c_{e_{2}}
$$

This is true because, graphically, the tangent line through $f$ at $c=c_{a}$ and $c=c_{b}$ is always below the curve $f$, by the nature of double-well potential. This says that, the assumption of hypothesis 2 gives lower energy than that of hypothesis 1 .

Thus, for $c_{e_{1}}<c_{o}<c_{e_{2}}$, or more clearly, for any metastable and unstable solutions, theoretically minimum energy is achieved when the local concentration of the entire solution is at either of equilibrium values, subject to mass conservation. This way, theoretical minimum energy can be quantitatively computed. Note however that, in the other section, growth factor of a mixture from uniformly mixed state is positive for unstable solution and negative for metastable solution. Due to partial-stability (metastability), hypothesis 1 is true for metastable solution, i.e. metastable solution has temporal minimum in energy when it is in uniformly mixed state, but the solution with equilibrium values everywhere still yields even lower energy. Thus, some kind of "activation" is required for phase separation in metastable solution.

On the other hand, if the solution has $c_{o}<c_{e_{1}}<c_{e_{2}}$ or $c_{e_{1}}<c_{e_{2}}<c_{o}$, the mass conservation used for hypothesis 2 cannot be satisfied.

Proof:

$$
\begin{gathered}
\left.\int_{\Omega_{e_{1}}} c\right|_{e_{1}} \partial v+\left.\int_{\Omega_{e_{2}}} c\right|_{e_{2}} \partial v>\int_{\Omega} c_{o} \partial v \text { if } c_{o}<c_{e_{1}}<c_{e_{2}} \\
\left.\int_{\Omega_{e_{1}}} c\right|_{e_{1}} \partial v+\left.\int_{\Omega_{e_{2}}} c\right|_{e_{2}} \partial v<\int_{\Omega} c_{o} \partial v \text { if } c_{e_{1}}<c_{e_{2}}<c_{o}
\end{gathered}
$$

This is a contradiction to mass conservation above. 
Thus, for stable mixture, hypothesis 1 is true. i.e. the energy of the mixture is minimized when solution is in uniformly mixed state.

\section{A.5 Dynamics of polymer mixture}

Potential difference of binary polymer solution can be obtained by taking functional derivative of the energy:

$$
\mu_{2}-\mu_{1}=\frac{\delta E}{\delta c}=\frac{\partial I}{\partial c}-\nabla \cdot \frac{\partial I}{\partial(\nabla c)}+\cdots=\frac{\partial f}{\partial c}-2 \kappa \nabla^{2} c
$$

Where $I$ is the integrant of $E$. Here, $f$ is obtained by Flory-Huggins' theory. Recall the assumption that $\kappa$ is a constant independent of concentration is made. This assumptions have been observed in multiple researches, and this still gives satisfactory results to fulfill the intentions of this thesis.

The mass flux within the solution can be obtained by Fick's law:

$$
\vec{J}=-M \nabla\left(\mu_{2}-\mu_{1}\right)
$$

Where $M$ is a positive mobility constant.

Here, another assumption is made, that $M$ is a positive constant, though it should be dependent on the concentration. However, this simplification enables us to compute expected characteristic frequency range throughout the domain, making the study more strategic and better infer the conclusions.

By the law of continuity:

$$
\frac{\partial c}{\partial t}=-\nabla \cdot \vec{J}=\nabla\left[M \nabla\left(\mu_{2}-\mu_{1}\right)\right]
$$

By combining equations above: 


$$
\frac{\partial c}{\partial t}=\nabla \cdot\left[M \nabla\left(\frac{\partial f}{\partial c}-2 \kappa \nabla^{2} c\right)\right]
$$

For a closed system, there must be no mass-flux at the boundaries:

$$
\vec{J} \cdot \hat{n}=0 \text { on } \partial \Omega
$$

This boundary condition ensures that mass conservation is satisfied.

Proof:

By divergence theorem and equations above, the change in the mass of the entire solution is:

$$
\frac{\partial}{\partial t} m=\frac{\partial}{\partial t} \int_{\Omega} c \partial v=\int_{\Omega} \frac{\partial c}{\partial t} \partial v=\int_{\Omega}(-\nabla \cdot \vec{J}) \partial v=-\left.\int_{\Omega}(\vec{J} \cdot \hat{n})\right|_{\partial \Omega} \partial v=0
$$

\section{QED}

For a variational problem, a wetting condition with no surface potential is also imposed:

$$
\nabla c \cdot \hat{n}=0 \text { on } \partial \Omega
$$

Because the energy of the solution has absolute gradient square term, this gives the structures at the boundary energy advantage.

Furthermore, the solution's dynamical change in morphology always starts from some configuration:

$$
\mathrm{c}(\vec{x}, t=0)=c_{o}
$$

Given boundary conditions above, Amy Novick-Cohen has shown that the solution is the problem in equation above is unique, and mass conservation is also satisfied. 


\section{A.6 Change of energy in binary polymer mixture}

Unless there is an external influence such as surface potential, thermodynamically, one may expect the change of energy in the solution to be zero or negative at all time as it approaches equilibrium state. In fact, the change in energy is the integral of squared absolute mass flux divided by positive mobility.

Proof:

By using chain rule and equations above, change of energy is:

$$
\frac{\partial E}{\partial t}=\frac{\partial}{\partial t} \int_{\Omega}\left(f+\kappa|\nabla c|^{2}\right) \partial v=\int_{\Omega}\left[\frac{\partial f}{\partial c} \frac{\partial c}{\partial t}+2 \kappa\left(\nabla c \cdot \nabla \frac{\partial c}{\partial t}\right)\right] \partial v
$$

By divergence theorem and zero-surface-potential boundary condition:

$$
\begin{aligned}
\int_{\Omega} \nabla c \cdot\left(\nabla \frac{\partial c}{\partial t}\right) & \partial v=\int_{\Omega} \nabla \cdot\left[(\nabla c) \frac{\partial c}{\partial t}\right] \partial v-\int_{\Omega} \nabla^{2} c \frac{\partial c}{\partial t} \partial v \\
& =\left.\int_{\Omega}\left[\frac{\partial c}{\partial t}(\nabla c \cdot \vec{n})\right]\right|_{\partial \Omega} \partial v-\int_{\Omega} \nabla^{2} c \frac{\partial c}{\partial t} \partial v=-\int_{\Omega} \nabla^{2} c \frac{\partial c}{\partial t} \partial v
\end{aligned}
$$

Using this, equation above can now be simplified. By further using divergence theorem once more and no mass flux boundary conditions: 


$$
\begin{aligned}
\frac{\partial E}{\partial t}=\int_{\Omega} \frac{\partial c}{\partial t}( & \left.\frac{\partial f}{\partial c}-2 \kappa \nabla^{2} c\right) \partial v=\int_{\Omega}(-\nabla \cdot \vec{J})\left(\mu_{2}-\mu_{1}\right) \partial v \\
& =-\int_{\Omega} \nabla \cdot\left[\left(\mu_{2}-\mu_{1}\right) \vec{J}\right] \partial v+\int_{\Omega} \vec{J} \cdot\left[\nabla\left(\mu_{2}-\mu_{1}\right)\right] \partial v \\
& =-\left.\int_{\Omega}\left[\left(\mu_{2}-\mu_{1}\right)(\vec{J} \cdot \vec{n})\right]\right|_{\partial \Omega} \partial v \\
& -\int_{\Omega}\left[M \nabla\left(\mu_{2}-\mu_{1}\right)\right] \cdot\left[\nabla\left(\mu_{2}-\mu_{1}\right)\right] \partial v \\
& =-\int_{\Omega} M\left|\nabla\left(\mu_{2}-\mu_{1}\right)\right|^{2} \partial v=-\int_{\Omega} \frac{|\vec{J}|^{2}}{M} \partial v \leq 0
\end{aligned}
$$

QED

\section{A.7 Basis functions in 1-d}

Cahn-Hilliard equation has up to fourth derivative, making it quite challenging to simulate, especially in the past, when computer technology was still not as advanced as it is today. To reduce computational cost, the method of Galerkin Finite element method has helped to reduce fourth order problem into essentially second order problem. Furthermore, recall that surface potential boundary condition must be imposed, and that such boundary has not been imposed during the course of model simplification, just like no mass flux boundary condition. To solve it, basis functions that are up to first order continuous, or $C^{1}$-continuous is desired. This way, the weights that account for up to first order derivatives are made available for computations.

If the local element has the width of one element, then it would contain 4 basis functions, accounting for weights of absolute value and first derivative at each end. Because each basis

function is required to have 4 constraints to it, $3^{\text {rd }}$ degree polynomial is required. In summary, the basis functions are:

Has the form of $3^{\text {rd }}$ degree polynomial, i.e. 


$$
\Phi_{\tau}^{\omega}=\sum_{i=1}^{4} c_{i} \alpha^{(i-1)} \text { where } c_{i} \in \mathbb{R}
$$

where $\omega=\{0,1\}$ is the orientation which dictates which end the weight is on. More specifically, if $\omega=0$, then the weight is at $\alpha=0$, while if $\omega=1$ then the weight is at $\alpha=1 . \tau=\{0,1\}$ is the type of basis function. If $\tau=0$, then the basis function has weight of the absolute value, else if $\tau=1$, then the basis function has weight of the first derivative.

With the definitions as above, the constraints are required such that:

$$
\begin{aligned}
\left.\Phi_{0}^{0}\right|_{\alpha=0}=1 & \left.\Phi_{0}^{1}\right|_{\alpha=0}=0 & \left.\Phi_{1}^{0}\right|_{\alpha=0}=0 & \left.\Phi_{1}^{1}\right|_{\alpha=0}=0 \\
\left.\Phi_{0}^{0}\right|_{\alpha=1}=0 & \left.\Phi_{0}^{1}\right|_{\alpha=1}=1 & \left.\Phi_{1}^{0}\right|_{\alpha=1}=0 & \left.\Phi_{1}^{1}\right|_{\alpha=1}=0 \\
\left.\frac{\partial}{\partial \alpha} \Phi_{0}^{0}\right|_{\alpha=0}=0 & \left.\frac{\partial}{\partial \alpha} \Phi_{0}^{1}\right|_{\alpha=0}=0 & \left.\frac{\partial}{\partial \alpha} \Phi_{1}^{0}\right|_{\alpha=0}=1 & \left.\frac{\partial}{\partial \alpha} \Phi_{1}^{1}\right|_{\alpha=0}=0 \\
\left.\frac{\partial}{\partial \alpha} \Phi_{0}^{0}\right|_{\alpha=1}=0 & \left.\frac{\partial}{\partial \alpha} \Phi_{0}^{1}\right|_{\alpha=1}=0 & \left.\frac{\partial}{\partial \alpha} \Phi_{1}^{0}\right|_{\alpha=1}=0 & \left.\frac{\partial}{\partial \alpha} \Phi_{1}^{1}\right|_{\alpha=1}=1
\end{aligned}
$$

Thus, 4 linear relationships for each basis function have been established. Upon solving it:

$$
\begin{gathered}
\Phi_{0}^{0}=2 \alpha^{3}-3 \alpha^{2}+1 \\
\Phi_{1}^{0}=\alpha^{3}-2 \alpha^{2}+\alpha \\
\Phi_{0}^{1}=-2 \alpha^{3}+3 \alpha^{2} \\
\Phi_{1}^{1}=\alpha^{3}-\alpha^{2}
\end{gathered}
$$

The weights within a local element are distributed as the summation of 4 polynomials as above. This way, the constraints at each node is preserved. This serves as basis functions:

$$
c(\alpha)=\sum_{\tau=\{0,1\}} \sum_{\omega=\{0,1\}} c_{\tau}^{\omega} \Phi_{\tau}^{\omega}
$$

Where $c_{\tau}^{\omega} \in \mathbb{R}$ is the corresponding weights. For example, the derivative with respect to $\alpha$ at the node $\alpha=1$ is $c_{1}^{1}$. 
These are four basis functions within local element and are commonly referred to as bicubic Hermitian functions, which arise often in nature, statistics, and engineering applications just like this thesis. Remarkable advantage is that, evaluation with 3 points Gaussian quadrature gives accurate integration. By using similar method, one may obtain Hermitian polynomials of higher degrees to make it $C^{2}, C^{3}, \ldots$-continuous for even more accurate results but at the expense of computational cost due to more constraints. For this thesis, bicubic Hermitian basis functions with $C^{1}$-continuous property will be suffice to satisfy the intent of this thesis.

\section{A.8 Bicubic Hermitian basis functions in 2-d}

Bicubic Hermitian basis functions are plain multiplications of 1-d Hermitian basis functions in each dimension, $\alpha$ and $\beta$, which are orthonormal to one another. This way, each element consists of 16 basis functions, and each node has 4 weights of types $\tau=\{00,10,01,11\}$

representing, in order, absolute value, first derivative with respect to $\alpha\left(\frac{\partial}{\partial \alpha}\right)$, first derivative with respect to $\beta\left(\frac{\partial}{\partial \beta}\right)$, and mixed derivative with respect to both $\left(\frac{\partial^{2}}{\partial \alpha \partial \beta}\right)$. Suppose $\omega=$ $\{00,10,01,11\}$ is the orientation which determines at which corner of the element the weight is in order $(\alpha, \beta)=(0,0),(1,0),(0,1),(1,1)$. This way, 16 local basis functions can be listed:

$$
\begin{gathered}
\Phi_{00}^{00}=\left(2 \alpha^{3}-3 \alpha^{2}+1\right)\left(2 \beta^{3}-3 \beta^{2}+1\right) \\
\Phi_{10}^{00}=\left(\alpha^{3}-2 \alpha^{2}+\alpha\right)\left(2 \beta^{3}-3 \beta^{2}+1\right) \\
\Phi_{01}^{00}=\left(2 \alpha^{3}-3 \alpha^{2}+1\right)\left(\beta^{3}-2 \beta^{2}+\beta\right) \\
\Phi_{11}^{00}=\left(\alpha^{3}-2 \alpha^{2}+\alpha\right)\left(\beta^{3}-2 \beta^{2}+\beta\right) \\
\Phi_{00}^{10}=\left(-2 \alpha^{3}+3 \alpha^{2}\right)\left(2 \beta^{3}-3 \beta^{2}+1\right) \\
\Phi_{10}^{10}=\left(\alpha^{3}-\alpha^{2}\right)\left(2 \beta^{3}-3 \beta^{2}+1\right) \\
\Phi_{01}^{10}=\left(-2 \alpha^{3}+3 \alpha^{2}\right)\left(\beta^{3}-2 \beta^{2}+\beta\right) \\
\Phi_{11}^{10}=\left(\alpha^{3}-\alpha^{2}\right)\left(\beta^{3}-2 \beta^{2}+\beta\right) \\
\Phi_{00}^{01}=\left(2 \alpha^{3}-3 \alpha^{2}+1\right)\left(-2 \beta^{3}+3 \beta^{2}\right) \\
\Phi_{10}^{01}=\left(\alpha^{3}-2 \alpha^{2}+\alpha\right)\left(-2 \beta^{3}+3 \beta^{2}\right) \\
\Phi_{01}^{01}=\left(2 \alpha^{3}-3 \alpha^{2}+1\right)\left(\beta^{3}-\beta^{2}\right) \\
\Phi_{11}^{01}=\left(\alpha^{3}-2 \alpha^{2}+\alpha\right)\left(\beta^{3}-\beta^{2}\right)
\end{gathered}
$$




$$
\begin{gathered}
\Phi_{00}^{11}=\left(-2 \alpha^{3}+3 \alpha^{2}\right)\left(-2 \beta^{3}+3 \beta^{2}\right) \\
\Phi_{10}^{11}=\left(\alpha^{3}-\alpha^{2}\right)\left(-2 \beta^{3}+3 \beta^{2}\right) \\
\Phi_{01}^{11}=\left(-2 \alpha^{3}+3 \alpha^{2}\right)\left(\beta^{3}-\beta^{2}\right) \\
\Phi_{11}^{11}=\left(\alpha^{3}-\alpha^{2}\right)\left(\beta^{3}-\beta^{2}\right)
\end{gathered}
$$

The weights within a local element is the summation of all polynomials above:

$$
c(\alpha, \beta)=\sum_{\tau=\{00,10,01,11\}} \sum_{\omega=\{00,10,01,11\}} c_{\tau}^{\omega} \Phi_{\tau}^{\omega} \text { where } c_{\tau}^{\omega} \in \mathbb{R}
$$

Again, these equations yield accurate 3-points Gaussian quadrature.

\section{A.9 Hermitian basis functions with serendipity in 3-d}

Bicubic Hermitian basis functions can be constructed in the same way as in 2-d. However, each node will now consist of 8 elements, and each now cubic element will consist of 64 basis functions. With reasonably fine mesh, this is computationally too expensive. Recall that imposing surface boundary conditions does not require mixed term. Not only that, when nonzero surface potential is imposed, the mixed term will cause some complications at the boundaries, because the variation of the first derivative is no longer constant, i.e. the mixed terms at the boundaries are no longer zero. Thus, one needs to resort to other type of basis functions for 3-d model with surface potential that do not contain mixed terms. Suppose each node of local element consists of 4 weights representing absolute value and the first derivatives with respect to each coordinate, which in local coordinates are $\alpha, \beta$, and $\gamma$, then each basis function within an element will now only 32 constraints. This brings significant reduction in computing power. Earlier, 1-d and 2-d basis functions with third degree polynomials have been derived. By following similar procedure: 


$$
\begin{aligned}
P(\alpha, \beta, \gamma)=( & \left.\sum_{i=1}^{4} c_{i} \alpha^{(i-1)}\right)\left(\sum_{j=1}^{4} c_{j} \beta^{(j-1)}\right)\left(\sum_{k=1}^{4} c_{k} \gamma^{(k-1)}\right) \\
& =c_{1} \alpha^{3} \beta^{3} \gamma^{3}+c_{2} \alpha^{3} \beta^{3} \gamma^{2}+c_{3} \alpha^{3} \beta^{3} \gamma+c_{4} \alpha^{3} \beta^{3}+c_{5} \alpha^{3} \beta^{2} \gamma^{3} \\
& +c_{6} \alpha^{3} \beta^{2} \gamma^{2}+c_{7} \alpha^{3} \beta^{2} \gamma+c_{8} \alpha^{3} \beta^{2}+c_{9} \alpha^{3} \beta \gamma^{3}+c_{10} \alpha^{3} \beta \gamma^{2} \\
& +c_{11} \alpha^{3} \beta \gamma+c_{12} \alpha^{3} \beta+c_{13} \alpha^{3} \gamma^{3}+c_{14} \alpha^{3} \gamma^{2}+c_{15} \alpha^{3} \gamma \\
& +c_{16} \alpha^{3}+c_{17} \alpha^{2} \beta^{3} \gamma^{3}+c_{18} \alpha^{2} \beta^{3} \gamma^{2}+c_{19} \alpha^{2} \beta^{3} \gamma+c_{20} \alpha^{2} \beta^{3} \\
& +c_{21} \alpha^{2} \beta^{2} \gamma^{3}+c_{22} \alpha^{2} \beta^{2} \gamma^{2}+c_{23} \alpha^{2} \beta^{2} \gamma+c_{24} \alpha^{2} \beta^{2} \\
& +c_{25} \alpha^{2} \beta \gamma^{3}+c_{26} \alpha^{2} \beta \gamma^{2}+c_{27} \alpha^{2} \beta \gamma+c_{28} \alpha^{2} \beta+c_{29} \alpha^{2} \gamma^{3} \\
& +c_{30} \alpha^{2} \gamma^{2}+c_{31} \alpha^{2} \gamma+c_{32} \alpha^{2}+c_{33} \alpha \beta^{3} \gamma^{3}+c_{34} \alpha \beta^{3} \gamma^{2} \\
& +c_{35} \alpha \beta^{3} \gamma+c_{36} \alpha \beta^{3}+c_{37} \alpha \beta^{2} \gamma^{3}+c_{38} \alpha \beta^{2} \gamma^{2}+c_{39} \alpha \beta^{2} \gamma \\
& +c_{40} \alpha \beta^{2}+c_{41} \alpha \beta \gamma^{3}+c_{42} \alpha \beta \gamma^{2}+c_{43} \alpha \beta \gamma+c_{44} \alpha \beta \\
& +c_{45} \alpha \gamma^{3}+c_{46} \alpha \gamma^{2}+c_{47} \alpha \gamma+c_{48} \alpha+c_{49} \beta^{3} \gamma^{3}+c_{50} \beta^{3} \gamma^{2} \\
& +c_{51} \beta^{3} \gamma+c_{52} \beta^{3}+c_{53} \beta^{2} \gamma^{3}+c_{54} \beta^{2} \gamma^{2}+c_{55} \beta^{2} \gamma+c_{56} \beta^{2} \\
& +c_{57} \beta \gamma^{3}+c_{58} \beta \gamma^{2}+c_{59} \beta \gamma+c_{60} \beta+c_{61} \gamma^{3}+c_{62} \gamma^{2}+c_{63} \gamma \\
& +c_{64}
\end{aligned}
$$

As shown above, there are 64 constraints if 3 bicubic Hermitian basis functions were multiplied. Given the reasoning above, constraints are now cut down to just 32. In order to ensure simplicity and maximize linearity, the terms in which no more than one parameter has a power of larger than 1 are kept. Thus, the polynomial of each basis function now becomes:

$$
\begin{aligned}
P(\alpha, \beta, \gamma)= & \Phi_{\tau}^{\omega} \\
& =c_{1}+c_{2} \alpha+c_{3} \beta+c_{4} \gamma+c_{5} \alpha^{2}+c_{6} \beta^{2}+c_{7} \gamma^{3}+c_{8} \alpha \beta \\
& +c_{9} \alpha \gamma+c_{10} \beta \gamma+c_{11} \alpha^{3}+c_{12} \beta^{3}+c_{13} \gamma^{3}+c_{14} \alpha^{2} \beta \\
& +c_{15} \alpha^{2} \gamma+c_{16} \alpha \beta^{2}+c_{17} \beta^{2} \gamma+c_{18} \alpha \gamma^{2}+c_{19} \beta \gamma^{2}+c_{20} \alpha \beta \gamma \\
& +c_{21} \alpha^{3} \beta+c_{22} \alpha^{3} \gamma+c_{23} \alpha \beta^{3}+c_{24} \beta^{3} \gamma+c_{25} \alpha \gamma^{3}+c_{26} \beta \gamma^{3} \\
& +c_{27} \alpha^{2} \beta \gamma+c_{28} \alpha \beta^{2} \gamma+c_{29} \alpha \beta \gamma^{2}+c_{30} \alpha^{3} \beta \gamma+c_{31} \alpha \beta^{3} \gamma \\
& +c_{32} \alpha \beta \gamma^{3}
\end{aligned}
$$


These are the formats of the basis functions. Now, the constraints are imposed in similar way as before, i.e.:

$$
\begin{aligned}
\left.\Phi_{000}^{000}\right|_{\alpha=0, \beta=0, \gamma=0} & =1 \\
\left.\Phi_{000}^{000}\right|_{\alpha=1, \beta=0, \gamma=0} & =0 \\
\left.\Phi_{000}^{000}\right|_{\alpha=0, \beta=1, \gamma=0} & =0 \\
\ldots & =0 \\
\left.\frac{\partial}{\partial \beta} \Phi_{010}^{011}\right|_{\alpha=0, \beta=1, \gamma=0} & =1 \\
\left.\frac{\partial}{\partial \beta} \Phi_{010}^{011}\right|_{\alpha=1, \beta=1, \gamma=0} & =1 \\
\left.\frac{\partial}{\partial \gamma} \Phi_{001}^{111}\right|_{\alpha=1, \beta=1, \gamma=1} & =1
\end{aligned}
$$

Where $\omega=\{000,100,010,110,001,101,011,111\}$ is the orientation of the basis function which determines at which node (corner) of the local cubic element the weight is on, and $\tau=$ $\{000,100,010,001\}$ is the type, which determines at which component the derivative has a weight of 1 . Note that each of the type has the weight of derivative in no more than 1 component, as the mixed terms have been eliminated. The solution to the problem of linear system of equations above with 32 constraints for each basis function is unique and can be determined easily. All the constants are computed and shown below:

\begin{tabular}{|c|c|c|c|c|c|c|c|c|c|c|c|c|c|c|c|c|}
\hline & $c_{1}$ & $c_{2}$ & $c_{3}$ & $c_{4}$ & $c_{5}$ & $c_{6}$ & $c_{7}$ & $c_{8}$ & $c_{9}$ & $c_{10}$ & $c_{11}$ & $c_{12}$ & $c_{13}$ & $c_{14}$ & $c_{15}$ & $c_{16}$ \\
\hline$\Phi_{000}^{000}$ & 1 & 0 & 0 & 0 & -3 & -3 & -3 & -1 & -1 & -1 & 2 & 2 & 2 & 3 & 3 & 3 \\
\hline$\Phi_{100}^{000}$ & 0 & 1 & 0 & 0 & -2 & 0 & 0 & -1 & -1 & 0 & 1 & 0 & 0 & 2 & 2 & 0 \\
\hline$\Phi_{010}^{000}$ & 0 & 0 & 1 & 0 & 0 & -2 & 0 & -1 & 0 & -1 & 0 & 1 & 0 & 0 & 0 & 2 \\
\hline$\Phi_{001}^{000}$ & 0 & 0 & 0 & 1 & 0 & 0 & -2 & 0 & -1 & -1 & 0 & 0 & 1 & 0 & 0 & 0 \\
\hline$\Phi_{000}^{100}$ & 0 & 0 & 0 & 0 & 3 & 0 & 0 & 1 & 1 & 0 & -2 & 0 & 0 & -3 & -3 & -3 \\
\hline$\Phi_{100}^{100}$ & 0 & 0 & 0 & 0 & -1 & 0 & 0 & 0 & 0 & 0 & 1 & 0 & 0 & 1 & 1 & 0 \\
\hline$\Phi_{010}^{100}$ & 0 & 0 & 0 & 0 & 0 & 0 & 0 & 1 & 0 & 0 & 0 & 0 & 0 & 0 & 0 & -2 \\
\hline
\end{tabular}




\begin{tabular}{|c|c|c|c|c|c|c|c|c|c|c|c|c|c|c|c|c|}
\hline$\Phi_{001}^{100}$ & 0 & 0 & 0 & 0 & 0 & 0 & 0 & 0 & 1 & 0 & 0 & 0 & 0 & 0 & 0 & 0 \\
\hline$\Phi_{000}^{010}$ & 0 & 0 & 0 & 0 & 0 & 3 & 0 & 1 & 0 & 1 & 0 & -2 & 0 & -3 & 0 & -3 \\
\hline$\Phi_{100}^{010}$ & 0 & 0 & 0 & 0 & 0 & 0 & 0 & 1 & 0 & 0 & 0 & 0 & 0 & -2 & 0 & 0 \\
\hline$\Phi_{010}^{010}$ & 0 & 0 & 0 & 0 & 0 & -1 & 0 & 0 & 0 & 0 & 0 & 1 & 0 & 0 & 0 & 1 \\
\hline$\Phi_{001}^{010}$ & 0 & 0 & 0 & 0 & 0 & 0 & 0 & 0 & 0 & 1 & 0 & 0 & 0 & 0 & 0 & 0 \\
\hline$\Phi_{000}^{110}$ & 0 & 0 & 0 & 0 & 0 & 0 & 0 & -1 & 0 & 0 & 0 & 0 & 0 & 3 & 0 & 3 \\
\hline$\Phi_{100}^{110}$ & 0 & 0 & 0 & 0 & 0 & 0 & 0 & 0 & 0 & 0 & 0 & 0 & 0 & -1 & 0 & 0 \\
\hline$\Phi_{010}^{110}$ & 0 & 0 & 0 & 0 & 0 & 0 & 0 & 0 & 0 & 0 & 0 & 0 & 0 & 0 & 0 & -1 \\
\hline$\Phi_{001}^{110}$ & 0 & 0 & 0 & 0 & 0 & 0 & 0 & 0 & 0 & 0 & 0 & 0 & 0 & 0 & 0 & 0 \\
\hline$\Phi_{000}^{001}$ & 0 & 0 & 0 & 0 & 0 & 0 & 3 & 0 & 1 & 1 & 0 & 0 & -2 & 0 & -3 & 0 \\
\hline$\Phi_{100}^{001}$ & 0 & 0 & 0 & 0 & 0 & 0 & 0 & 0 & 1 & 0 & 0 & 0 & 0 & 0 & -2 & 0 \\
\hline$\Phi_{010}^{001}$ & 0 & 0 & 0 & 0 & 0 & 0 & 0 & 0 & 0 & 1 & 0 & 0 & 0 & 0 & 0 & 0 \\
\hline$\Phi_{001}^{001}$ & 0 & 0 & 0 & 0 & 0 & 0 & -1 & 0 & 0 & 0 & 0 & 0 & 1 & 0 & 0 & 0 \\
\hline$\Phi_{000}^{101}$ & 0 & 0 & 0 & 0 & 0 & 0 & 0 & 0 & -1 & 0 & 0 & 0 & 0 & 0 & 3 & 0 \\
\hline$\Phi_{100}^{101}$ & 0 & 0 & 0 & 0 & 0 & 0 & 0 & 0 & 0 & 0 & 0 & 0 & 0 & 0 & -1 & 0 \\
\hline$\Phi_{010}^{101}$ & 0 & 0 & 0 & 0 & 0 & 0 & 0 & 0 & 0 & 0 & 0 & 0 & 0 & 0 & 0 & 0 \\
\hline$\Phi_{001}^{101}$ & 0 & 0 & 0 & 0 & 0 & 0 & 0 & 0 & 0 & 0 & 0 & 0 & 0 & 0 & 0 & 0 \\
\hline$\Phi_{000}^{011}$ & 0 & 0 & 0 & 0 & 0 & 0 & 0 & 0 & 0 & -1 & 0 & 0 & 0 & 0 & 0 & 0 \\
\hline$\Phi_{100}^{011}$ & 0 & 0 & 0 & 0 & 0 & 0 & 0 & 0 & 0 & 0 & 0 & 0 & 0 & 0 & 0 & 0 \\
\hline$\Phi_{010}^{011}$ & 0 & 0 & 0 & 0 & 0 & 0 & 0 & 0 & 0 & 0 & 0 & 0 & 0 & 0 & 0 & 0 \\
\hline$\Phi_{001}^{011}$ & 0 & 0 & 0 & 0 & 0 & 0 & 0 & 0 & 0 & 0 & 0 & 0 & 0 & 0 & 0 & 0 \\
\hline$\Phi_{000}^{111}$ & 0 & 0 & 0 & 0 & 0 & 0 & 0 & 0 & 0 & 0 & 0 & 0 & 0 & 0 & 0 & 0 \\
\hline$\Phi_{100}^{111}$ & 0 & 0 & 0 & 0 & 0 & 0 & 0 & 0 & 0 & 0 & 0 & 0 & 0 & 0 & 0 & 0 \\
\hline$\Phi_{010}^{111}$ & 0 & 0 & 0 & 0 & 0 & 0 & 0 & 0 & 0 & 0 & 0 & 0 & 0 & 0 & 0 & 0 \\
\hline$\Phi_{001}^{111}$ & 0 & 0 & 0 & 0 & 0 & 0 & 0 & 0 & 0 & 0 & 0 & 0 & 0 & 0 & 0 & 0 \\
\hline
\end{tabular}

\begin{tabular}{|c|c|c|c|c|c|c|c|c|c|c|c|c|c|c|c|c|}
\hline & $c_{17}$ & $c_{18}$ & $c_{19}$ & $c_{20}$ & $c_{21}$ & $c_{22}$ & $c_{23}$ & $c_{24}$ & $c_{25}$ & $c_{26}$ & $c_{27}$ & $c_{28}$ & $c_{29}$ & $c_{30}$ & $c_{31}$ & $c_{32}$ \\
\hline$\Phi_{000}^{000}$ & 3 & 3 & 3 & 2 & -2 & -2 & -2 & -2 & -2 & -2 & -3 & -3 & -3 & 2 & 2 & 2 \\
\hline$\Phi_{100}^{000}$ & 0 & 0 & 0 & 1 & -1 & -1 & 0 & 0 & 0 & 0 & -2 & 0 & 0 & 1 & 0 & 0 \\
\hline
\end{tabular}




\begin{tabular}{|c|c|c|c|c|c|c|c|c|c|c|c|c|c|c|c|c|}
\hline$\Phi_{010}^{000}$ & 2 & 0 & 0 & 1 & 0 & 0 & -1 & -1 & 0 & 0 & 0 & -2 & 0 & 0 & 1 & 0 \\
\hline$\Phi_{001}^{000}$ & 0 & 2 & 2 & 1 & 0 & 0 & 0 & 0 & -1 & -1 & 0 & 0 & -2 & 0 & 0 & 1 \\
\hline$\Phi_{000}^{100}$ & 0 & -3 & 0 & -2 & 2 & 2 & 2 & 0 & 2 & 0 & 3 & 3 & 3 & -2 & -2 & -2 \\
\hline$\Phi_{100}^{100}$ & 0 & 0 & 0 & 0 & -1 & -1 & 0 & 0 & 0 & 0 & -1 & 0 & 0 & 1 & 0 & 0 \\
\hline$\Phi_{010}^{100}$ & 0 & 0 & 0 & -1 & 0 & 0 & 1 & 0 & 0 & 0 & 0 & 2 & 0 & 0 & -1 & 0 \\
\hline$\Phi_{001}^{100}$ & 0 & -2 & 0 & -1 & 0 & 0 & 0 & 0 & 1 & 0 & 0 & 0 & 2 & 0 & 0 & -1 \\
\hline$\Phi_{000}^{010}$ & -3 & 0 & -3 & -2 & 2 & 0 & 2 & 2 & 0 & 2 & 3 & 3 & 3 & -2 & -2 & -2 \\
\hline$\Phi_{100}^{010}$ & 0 & 0 & 0 & -1 & 1 & 0 & 0 & 0 & 0 & 0 & 2 & 0 & 0 & -1 & 0 & 0 \\
\hline$\Phi_{010}^{010}$ & 1 & 0 & 0 & 0 & 0 & 0 & -1 & -1 & 0 & 0 & 0 & -1 & 0 & 0 & 1 & 0 \\
\hline$\Phi_{001}^{010}$ & 0 & 0 & -2 & -1 & 0 & 0 & 0 & 0 & 0 & 1 & 0 & 0 & 2 & 0 & 0 & -1 \\
\hline$\Phi_{000}^{110}$ & 0 & 0 & 0 & 2 & -2 & 0 & -2 & 0 & 0 & 0 & -3 & -3 & -3 & 2 & 2 & 2 \\
\hline$\Phi_{100}^{110}$ & 0 & 0 & 0 & 0 & 1 & 0 & 0 & 0 & 0 & 0 & 1 & 0 & 0 & -1 & 0 & 0 \\
\hline$\Phi_{010}^{110}$ & 0 & 0 & 0 & 0 & 0 & 0 & 1 & 0 & 0 & 0 & 0 & 1 & 0 & 0 & -1 & 0 \\
\hline$\Phi_{001}^{110}$ & 0 & 0 & 0 & 1 & 0 & 0 & 0 & 0 & 0 & 0 & 0 & 0 & -2 & 0 & 0 & 1 \\
\hline$\Phi_{000}^{001}$ & -3 & -3 & -3 & -2 & 0 & 2 & 0 & 2 & 2 & 2 & 3 & 3 & 3 & -2 & -2 & -2 \\
\hline$\Phi_{100}^{001}$ & 0 & 0 & 0 & -1 & 0 & 1 & 0 & 0 & 0 & 0 & 2 & 0 & 0 & -1 & 0 & 0 \\
\hline$\Phi_{010}^{001}$ & -2 & 0 & 0 & -1 & 0 & 0 & 0 & 1 & 0 & 0 & 0 & 2 & 0 & 0 & -1 & 0 \\
\hline$\Phi_{001}^{001}$ & 0 & 1 & 1 & 0 & 0 & 0 & 0 & 0 & -1 & -1 & 0 & 0 & -1 & 0 & 0 & 1 \\
\hline$\Phi_{000}^{101}$ & 0 & 3 & 0 & 2 & 0 & -2 & 0 & 0 & -2 & 0 & -3 & -3 & -3 & 2 & 2 & 2 \\
\hline$\Phi_{100}^{101}$ & 0 & 0 & 0 & 0 & 0 & 1 & 0 & 0 & 0 & 0 & 1 & 0 & 0 & -1 & 0 & 0 \\
\hline$\Phi_{010}^{101}$ & 0 & 0 & 0 & 1 & 0 & 0 & 0 & 0 & 0 & 0 & 0 & -2 & 0 & 0 & 1 & 0 \\
\hline$\Phi_{001}^{101}$ & 0 & -1 & 0 & 0 & 0 & 0 & 0 & 0 & 1 & 0 & 0 & 0 & 1 & 0 & 0 & -1 \\
\hline$\Phi_{000}^{011}$ & 3 & 0 & 3 & 2 & 0 & 0 & 0 & -2 & 0 & -2 & -3 & -3 & -3 & 2 & 2 & 2 \\
\hline$\Phi_{100}^{011}$ & 0 & 0 & 0 & 1 & 0 & 0 & 0 & 0 & 0 & 0 & -2 & 0 & 0 & 1 & 0 & 0 \\
\hline$\Phi_{010}^{011}$ & -1 & 0 & 0 & 0 & 0 & 0 & 0 & 1 & 0 & 0 & 0 & 1 & 0 & 0 & -1 & 0 \\
\hline$\Phi_{001}^{011}$ & 0 & 0 & -1 & 0 & 0 & 0 & 0 & 0 & 0 & 1 & 0 & 0 & 1 & 0 & 0 & -1 \\
\hline$\Phi_{000}^{111}$ & 0 & 0 & 0 & -2 & 0 & 0 & 0 & 0 & 0 & 0 & 3 & 3 & 3 & -2 & -2 & -2 \\
\hline$\Phi_{100}^{111}$ & 0 & 0 & 0 & 0 & 0 & 0 & 0 & 0 & 0 & 0 & -1 & 0 & 0 & 1 & 0 & 0 \\
\hline$\Phi_{010}^{111}$ & 0 & 0 & 0 & 0 & 0 & 0 & 0 & 0 & 0 & 0 & 0 & -1 & 0 & 0 & 1 & 0 \\
\hline
\end{tabular}




\begin{tabular}{|l|l|l|l|l|l|l|l|l|l|l|l|l|l|l|l|l|}
\hline$\Phi_{001}^{111}$ & 0 & 0 & 0 & 0 & 0 & 0 & 0 & 0 & 0 & 0 & 0 & 0 & -1 & 0 & 0 & 1 \\
\hline
\end{tabular}

Just as before, the weights within an element is distributed as a summation of all local basis functions above.

$$
\begin{aligned}
& c(\alpha, \beta, \gamma) \\
& =\sum_{\tau=\{000,100,010,001\}} \sum_{\omega=\{000,100,010,110,001,101,011,111\}} c_{\tau}^{\omega} \Phi_{\tau}^{\omega} \text { where } c_{\tau}^{\omega} \in \mathbb{R}
\end{aligned}
$$

Thus, basis functions with serendipity for 3-d have been derived, effectively saving tremendous computational cost. The value of it also lies within its nature wherein integral evaluation with Gaussian quadrature gives accurate results.

\section{A.10 Gaussian quadrature and isoparametric mapping in 1-d}

3-points Gaussian quadrature in 1-d

Conventionally, for some real function $f$, Gaussian quadrature is evaluated as following:

$$
\int_{-1}^{1} f(x) \partial x=\sum_{i=1}^{3} w_{i} f\left(x_{i}\right)
$$

Where:

$$
\begin{array}{r}
\left(x_{1}, x_{2}, x_{3}\right)=\left(-\sqrt{\frac{3}{5}}, 0, \sqrt{\frac{3}{5}}\right) \\
\left(w_{1}, w_{2}, w_{3}\right)=\left(\frac{5}{9}, \frac{8}{9}, \frac{5}{9}\right)
\end{array}
$$

In previous chapters, the basis functions within the domain that extends from $\alpha=0$ to $\alpha=1$ were derived. Now the integral's boundaries need to be adjusted accordingly. 
The domain the expands from -1 to 1 can be mapped into the domain that expands from 0 to 1 as following:

$$
\alpha=\frac{x+1}{2} \Leftrightarrow x=2 \alpha-1
$$

Integration obeys the chain rule for isoparametric mapping:

$$
\int_{0}^{1} f(\alpha) \partial \alpha=\int_{-1}^{1} f(\alpha(x)) \frac{\partial \alpha}{\partial x} \partial x=\frac{1}{2} \int_{-1}^{1} f(\alpha(x)) \partial x=\sum_{i=1}^{3} w_{i}^{*} f\left(\alpha_{i}\right)
$$

Where:

$$
\begin{gathered}
\left(w_{1}^{*}, w_{2}^{*}, w_{3}^{*}\right)=\left(\frac{5}{18}, \frac{4}{9}, \frac{5}{18}\right) \\
\left(\alpha_{1}, \alpha_{2}, \alpha_{3}\right)=\left(\frac{-\sqrt{\frac{3}{5}}+1}{2}, \frac{1}{2}, \frac{\sqrt{\frac{3}{5}}+1}{2}\right)
\end{gathered}
$$

This is Guassian quadrature for local basis functions. Now, global coordinates need to be converted into local coordinates in order to give us the freedom of choosing mesh. The mapping is as following:

Suppose the global element spans from $x_{1}$ to $x_{2}$, then:

$$
\alpha=\frac{x-x_{1}}{\Delta x} \Leftrightarrow x=\Delta x \alpha+x_{1}
$$

Where:

$$
\Delta x=x_{2}-x_{1}
$$

Now, the remaining derivation is similar as above: 


$$
\begin{aligned}
\left.\int_{x_{1}}^{x_{2}} f(x)\right) \partial x & =\int_{0}^{1} f(x(\alpha)) \frac{\partial x}{\partial \alpha} \partial \alpha=\Delta x \int_{0}^{1} f(x(\alpha)) \partial \alpha \\
& =\Delta x \sum_{i=1}^{3} w_{i}^{*} f\left(\alpha_{i}\right)
\end{aligned}
$$

Where $w_{i}^{*}$ and $\alpha_{i}$ are the same as (A.10.3).

\section{A.11 Gaussian quadrature in 2-d and 3-d}

Gaussian quadrature in higher dimensions are easy if each of the component (i.e. $x, y, \ldots$ ) is orthonormal to all others. These are the cases in which each mesh is in rectangular shape for 2$d$ or rectangular prism shape for 3-d. However, if one is to use coordinates in which each component is not orthonormal from one another, which can arise from curved boundaries, it is a more complicated process. Here, the general method to convert global to local coordinates is shown.

First, let us consider 2-d case. Three points 2-d Gaussian quadrature is, by convention:

$$
\int_{-1}^{1} \int_{-1}^{1} f(x, y) \partial x \partial y=\sum_{i=1}^{3} \sum_{j=1}^{3} w_{i} w_{j} f\left(x_{i}, y_{j}\right)
$$

Where:

$$
\begin{aligned}
\left(x_{1}, x_{2}, x_{3}\right)=\left(y_{1}, y_{2}, y_{3}\right) & =\left(-\sqrt{\frac{3}{5}}, 0, \sqrt{\frac{3}{5}}\right) \\
\left(w_{1}, w_{2}, w_{3}\right) & =\left(\frac{5}{9}, \frac{8}{9}, \frac{5}{9}\right)
\end{aligned}
$$

Just as before, local basis function spans from value of 0 to 1 in each othonormal component. By following the similar derivations, the integration within the region of the local basis functions can be derived. Given: 


$$
\alpha=\frac{x+1}{2} \Leftrightarrow x=2 \alpha-1 \text { and } \beta=\frac{y+1}{2} \Leftrightarrow y=2 \beta-1
$$

Then:

$$
\begin{aligned}
\int_{0}^{1} \int_{0}^{1} f(\alpha, \beta) \partial \alpha \partial \beta & =\int_{-1}^{1} \int_{-1}^{1} f(\alpha(x, y), \beta(x, y)) J \partial x \partial y \\
= & \frac{1}{4} \int_{-1}^{1} \int_{-1}^{1} f(\alpha(x), \beta(y)) \partial x \partial y=\sum_{i=1}^{3} \sum_{j=1}^{3} w_{i}^{*} w_{j}^{*} f\left(\alpha_{i}, \beta_{j}\right)
\end{aligned}
$$

Where:

$$
\begin{gathered}
\left(w_{1}^{*}, w_{2}^{*}, w_{3}^{*}\right)=\left(\frac{5}{18}, \frac{4}{9}, \frac{5}{18}\right) \\
\left(\alpha_{1}, \alpha_{2}, \alpha_{3}\right)=\left(\beta_{1}, \beta_{2}, \beta_{3}\right)=\left(\frac{-\sqrt{\frac{3}{5}}+1}{2}, \frac{1}{2}, \frac{\sqrt{\frac{3}{5}}+1}{2}\right) \\
J=\left|\begin{array}{ll}
\frac{\partial \alpha}{\partial x} & \frac{\partial \alpha}{\partial y} \\
\frac{\partial \beta}{\partial x} & \frac{\partial \beta}{\partial y}
\end{array}\right|=\frac{\partial \alpha}{\partial x} \frac{\partial \beta}{\partial y}=\frac{1}{4}
\end{gathered}
$$

This is how integration of local basis element is evaluated. Now, global basis functions must be converted into local basis functions.

$$
\begin{array}{r}
\int_{y_{1}}^{y_{2}} \int_{x_{1}}^{x_{2}} f(x, y) \partial x \partial y=\int_{0}^{1} \int_{0}^{1} f(x(\alpha, \beta), y(\alpha, \beta)) J \partial \alpha \partial \beta \\
=\sum_{i=1}^{3} \sum_{j=1}^{3} w_{i}^{*} w_{j}^{*} J\left(\alpha_{i}, \beta_{j}\right) f\left(x\left(\alpha_{i}, \beta_{j}\right), x\left(\alpha_{i}, \beta_{j}\right)\right)
\end{array}
$$

Where:

$$
J\left(\alpha_{i}, \beta_{j}\right)=\left|\begin{array}{ll}
\frac{\partial x}{\partial \alpha} & \frac{\partial x}{\partial \beta} \\
\frac{\partial y}{\partial \alpha} & \frac{\partial y}{\partial \beta}
\end{array}\right|
$$


Where $\left(\alpha_{1}, \alpha_{2}, \alpha_{3}\right),\left(\beta_{1}, \beta_{2}, \beta_{3}\right)$, and $\left(w_{1}^{*}, w_{2}^{*}, w_{3}^{*}\right)$ are same as above.

For rectangular element, the integration is simple since the change of variables is as following:

$$
\alpha=\frac{x-x_{1}}{\Delta x} \Leftrightarrow x=\Delta x \alpha+x_{1} \text { and } \beta=\frac{y-y_{1}}{\Delta y} \Leftrightarrow y=\Delta y \beta+y_{1}
$$

Where:

$$
\Delta x=x_{2}-x_{1} \text { and } \Delta y=y_{2}-y_{1}
$$

Thus:

$$
\mathrm{J}=\Delta x \Delta y
$$

And:

$$
\int_{y_{1}}^{y_{2}} \int_{x_{1}}^{x_{2}} f(x, y) \partial x \partial y=\Delta x \Delta y \sum_{i=1}^{3} \sum_{j=1}^{3} w_{i}^{*} w_{j}^{*} f\left(x\left(\alpha_{i}\right), y\left(\beta_{j}\right)\right)
$$

Gaussian quadrature in 3-d is derived the same way.

$$
\begin{aligned}
& \int_{z_{1}}^{z_{2}} \int_{y_{1}}^{y_{2}} \int_{x_{1}}^{x_{2}} f(x, y) \partial x \partial y \partial z \\
& =\sum_{k=1}^{3} \sum_{j=1}^{3} \sum_{i=1}^{3} w_{i}^{*} w_{j}^{*} w_{k}^{*} J\left(\alpha_{i}, \beta_{j}, \gamma_{k}\right) f\left(x\left(\alpha_{i}, \beta_{j}, \gamma_{k}\right), y\left(\alpha_{i}, \beta_{j}, \gamma_{k}\right), z\left(\alpha_{i}, \beta_{j}, \gamma_{k}\right)\right)
\end{aligned}
$$

Where:

$$
J\left(\alpha_{i}, \beta_{j}, \gamma_{k}\right)=\left|\begin{array}{lll}
\frac{\partial x}{\partial \alpha} & \frac{\partial x}{\partial \beta} & \frac{\partial x}{\partial \gamma} \\
\frac{\partial y}{\partial \alpha} & \frac{\partial y}{\partial \beta} & \frac{\partial y}{\partial \gamma} \\
\frac{\partial z}{\partial \beta} & \frac{\partial z}{\partial \beta} & \frac{\partial z}{\partial \gamma}
\end{array}\right|
$$


And $\left(\alpha_{1}, \alpha_{2}, \alpha_{3}\right),\left(\beta_{1}, \beta_{2}, \beta_{3}\right),\left(\gamma_{1}, \gamma_{2}, \gamma_{3}\right)$, and $\left(w_{1}^{*}, w_{2}^{*}, w_{3}^{*}\right)$ are same as above. For rectangular prism, the equation simplifies to:

$$
\begin{aligned}
& \int_{z_{1}}^{z_{2}} \int_{y_{1}}^{y_{2}} \int_{x_{1}}^{x_{2}} f(x, y, z) \partial x \partial y \partial z \\
& \quad=\Delta x \Delta y \Delta z \sum_{k=1}^{3} \sum_{j=1}^{3} \sum_{i=1}^{3} w_{i}^{*} w_{j}^{*} w_{k}^{*} f\left(x\left(\alpha_{i}\right), y\left(\beta_{j}\right), z\left(\gamma_{k}\right)\right)
\end{aligned}
$$

Now that the method for evaluating the integral of global coordinates in local coordinates has been shown, in the next section, the type of transformations that are selected for this thesis is explicitly explained.

\section{A.12 Isoparametric mapping of global basis functions from local basis functions}

In the previous sections, it has shown how to evaluate integrals of global basis functions in local basis functions. This requires one critical step: transformation of global basis functions to local basis functions. In section is intended for tackling this problem.

In most of this thesis, phase separation in square domain is investigated most of the time. Normally, for rectangular domain, since the edges are straight, the simplest type of isoparametric mapping is suffice: linear isoparametric mapping. This is computationally cheapest, yet can yield reasonable results for intended purpose. For curved boundary domains, different types of mapping is typically recommended in other to account for the weights in the regions other than nodal points. For this section, the mapping in 2-d is demonstrated. The mapping in 3-d follows similar way.

In linear isoparametric mapping, quadrilateral with 4 corners, $\left(x_{1}, y_{1}\right),\left(x_{2}, y_{2}\right),\left(x_{3}, y_{3}\right)$, and $\left(x_{4}, y_{4}\right)$, are mapped from local element as following:

$$
x=x_{1}(1-\alpha)(1-\beta)+x_{2} \alpha(1-\beta)+x_{3}(1-\alpha) \beta+x_{4} \alpha \beta
$$




$$
y=y_{1}(1-\alpha)(1-\beta)+y_{2} \alpha(1-\beta)+y_{3}(1-\alpha) \beta+y_{4} \alpha \beta
$$

Though straightforward, for the ease of discussion, the derivatives are listed here:

$$
\begin{gathered}
\frac{\partial x}{\partial \alpha}=-x_{1}(1-\beta)+x_{2}(1-\beta)-x_{3} \beta+x_{4} \beta \\
\frac{\partial x}{\partial \beta}=-x_{1}(1-\alpha)-x_{2} \alpha+x_{3}(1-\alpha)+x_{4} \alpha \\
\frac{\partial y}{\partial \alpha}=-y_{1}(1-\beta)+y_{2}(1-\beta)-y_{3} \beta+y_{4} \beta \\
\frac{\partial y}{\partial \beta}=-y_{1}(1-\alpha)-y_{2} \alpha+y_{3}(1-\alpha)+y_{4} \alpha \\
\frac{\partial^{2} x}{\partial \alpha \partial \beta}=x_{1}-x_{2}-x_{3}+x_{4} \\
\frac{\partial^{2} y}{\partial \alpha \partial \beta}=y_{1}-y_{2}-y_{3}+y_{4} \\
\frac{\partial^{2} x}{\partial \alpha^{2}}=\frac{\partial^{2} x}{\partial \beta^{2}}=\frac{\partial^{3} x}{\partial \alpha^{2} \partial \beta}=\cdots=0
\end{gathered}
$$

The type of mapping has been selected, next how basis functions can map is demonstrated. In an excellent textbook by Leon Lapidus [78], Hermitian basis functions in 2-d can be mapped from local coordinate to global coordinate as following:

$$
\begin{gathered}
\varphi_{00}^{\omega}=\Phi_{00}^{\omega} \\
\varphi_{10}^{\omega}=\Phi_{10}^{\omega} \frac{\partial x}{\partial \alpha}+\Phi_{01}^{\omega} \frac{\partial x}{\partial \beta}+\Phi_{11}^{\omega} \frac{\partial^{2} x}{\partial \alpha \partial \beta} \\
\varphi_{01}^{\omega}=\Phi_{10}^{\omega} \frac{\partial y}{\partial \alpha}+\Phi_{01}^{\omega} \frac{\partial y}{\partial \beta}+\Phi_{11}^{\omega} \frac{\partial^{2} y}{\partial \alpha \partial \beta} \\
\varphi_{11}^{\omega}=\Phi_{11}^{\omega}\left(\frac{\partial x}{\partial \beta} \frac{\partial y}{\partial \alpha}+\frac{\partial x}{\partial \alpha} \frac{\partial y}{\partial \beta}\right) \\
\varphi_{20}^{\omega}=\Phi_{11}^{\omega} \frac{\partial x}{\partial \alpha} \frac{\partial x}{\partial \beta} \\
\varphi_{02}^{\omega}=\Phi_{11}^{\omega} \frac{\partial y}{\partial \alpha} \frac{\partial y}{\partial \beta}
\end{gathered}
$$


There are 6 constraints in total. $\varphi_{20}^{\omega}$ and $\varphi_{02}^{\omega}$ represent the basis functions that account for second derivative with respect to $x$ and $y$, respectively. For a rectangular mesh, mapping of global coordinate to local coordinate is a lot simplified since $x_{1}=x_{3}, x_{2}=x_{4}, y_{1}=y_{2}$, and $y_{3}=y_{4}$. Supposed global coordinate has a width of $\Delta x=x_{2}-x_{1}$ and height of $\Delta y=y_{3}-y_{1}$, then:

$$
\begin{gathered}
\varphi_{00}^{\omega}=\Phi_{00}^{\omega} \\
\varphi_{10}^{\omega}=\Delta x \Phi_{10}^{\omega} \\
\varphi_{01}^{\omega}=\Delta y \Phi_{01}^{\omega} \\
\varphi_{11}^{\omega}=\Delta x \Delta y \Phi_{11}^{\omega}
\end{gathered}
$$

For governing equation, one needs to be able to evaluate several derivatives of the concentration in global coordinate. Such evaluation can be done by taking advantage of the relationship of global and local coordinates using chain rules. By chain rule:

$$
\left(\begin{array}{c}
\frac{\partial \varphi}{\partial a} \\
\frac{\partial \varphi}{\partial \beta} \\
\frac{\partial^{2} \varphi}{\partial a^{2}} \\
\frac{\partial^{2} \varphi}{\partial \beta^{2}} \\
\frac{\partial^{2} \varphi}{\partial \alpha \partial \beta}
\end{array}\right)=M_{\text {chain }}\left(\begin{array}{c}
\frac{\partial \varphi}{\partial x} \\
\frac{\partial \varphi}{\partial y} \\
\frac{\partial^{2} \varphi}{\partial x^{2}} \\
\frac{\partial^{2} \varphi}{\partial y^{2}} \\
\frac{\partial^{2} \varphi}{\partial x \partial y}
\end{array}\right)
$$

Where: 


$$
M_{\text {chain }}=\left[\begin{array}{ccccc}
\frac{\partial x}{\partial \alpha} & \frac{\partial y}{\partial \alpha} & 0 & 0 & 0 \\
\frac{\partial x}{\partial \beta} & \frac{\partial y}{\partial \beta} & 0 & 0 & 0 \\
\frac{\partial^{2} x}{\partial \alpha^{2}} & \frac{\partial^{2} y}{\partial \alpha^{2}} & \left(\frac{\partial x}{\partial \alpha}\right)^{2} & \left(\frac{\partial y}{\partial \alpha}\right)^{2} & 2 \frac{\partial x}{\partial \alpha} \frac{\partial y}{\partial \alpha} \\
\frac{\partial^{2} x}{\partial \beta^{2}} & \frac{\partial^{2} y}{\partial \beta^{2}} & \left(\frac{\partial x}{\partial \beta}\right)^{2} & \left(\frac{\partial y}{\partial \beta}\right)^{2} & 2 \frac{\partial x}{\partial \beta} \frac{\partial y}{\partial \beta} \\
\frac{\partial^{2} x}{\partial \alpha \partial \beta} & \frac{\partial^{2} y}{\partial \alpha \partial \beta} & \frac{\partial x}{\partial \alpha} \frac{\partial x}{\partial \beta} & \frac{\partial y}{\partial \alpha} \frac{\partial y}{\partial \beta} & \frac{\partial x}{\partial \alpha} \frac{\partial y}{\partial \beta}+\frac{\partial x}{\partial \beta} \frac{\partial y}{\partial \alpha}
\end{array}\right]
$$

By inverting $M_{\text {chain }}$, one can evaluate required derivatives:

$$
\left(\begin{array}{c}
\frac{\partial \varphi}{\partial x} \\
\frac{\partial \varphi}{\partial y} \\
\frac{\partial \varphi}{\partial x^{2}} \\
\frac{\partial^{2} \varphi}{\partial y^{2}} \\
\frac{\partial^{2} \varphi}{\partial x \partial y}
\end{array}\right)=M_{\text {chain }}^{-1}\left(\begin{array}{c}
\frac{\partial \varphi}{\partial a} \\
\frac{\partial \varphi}{\partial \beta} \\
\frac{\partial^{2} \varphi}{\partial a^{2}} \\
\frac{\partial^{2} \varphi}{\partial \beta^{2}} \\
\frac{\partial^{2} \varphi}{\partial \alpha \partial \beta}
\end{array}\right)
$$

The derivatives of global basis functions with respect to component(s) of local coordinates is done using the transformations listed above. For a rectangular element, this problem is much simplified, i.e.

$$
\begin{gathered}
\frac{\partial}{\partial \alpha} \varphi_{00}^{\omega}=\frac{\partial}{\partial \alpha} \Phi_{00}^{\omega} \\
\frac{\partial}{\partial \beta} \varphi_{00}^{\omega}=\frac{\partial}{\partial \beta} \Phi_{00}^{\omega} \\
\cdots \\
\frac{\partial^{2}}{\partial \alpha^{2}} \varphi_{10}^{\omega}=\Delta x \frac{\partial^{2}}{\partial \alpha^{2}} \Phi_{10}^{\omega}
\end{gathered}
$$




$$
\frac{\partial^{2}}{\partial \alpha \partial \beta} \varphi_{11}^{\omega}=\Delta x \Delta y \frac{\partial^{2}}{\partial \alpha \partial \beta} \Phi_{11}^{\omega}
$$

Where the local basis functions are as defined in previous chapters. One can also realize that $M_{\text {chain }}$ becomes simple diagonal matrix in rectangular mesh. Eventually, one can solve, for rectangular mesh:

$$
\begin{array}{ccccc}
\frac{\partial \varphi_{00}^{\omega}}{\partial x}=\frac{1}{\Delta x} \frac{\partial \Phi_{00}^{\omega}}{\partial \alpha} & \frac{\partial \varphi_{10}^{\omega}}{\partial x}=\frac{\partial \Phi_{10}^{\omega}}{\partial \alpha} & \frac{\partial \varphi_{01}^{\omega}}{\partial x}=\frac{\Delta y}{\Delta x} \frac{\partial \Phi_{01}^{\omega}}{\partial \alpha} & \frac{\partial \varphi_{11}^{\omega}}{\partial x}=\Delta y \frac{\partial \Phi_{11}^{\omega}}{\partial \alpha} \\
\frac{\partial \varphi_{00}^{\omega}}{\partial y}=\frac{1}{\Delta y} \frac{\partial \Phi_{00}^{\omega}}{\partial \beta} & \frac{\partial \varphi_{10}^{\omega}}{\partial y}=\frac{\Delta x}{\Delta y} \frac{\partial \Phi_{10}^{\omega}}{\partial \beta} & \frac{\partial \varphi_{01}^{\omega}}{\partial y}=\frac{\partial \Phi_{01}^{\omega}}{\partial \beta} & \frac{\partial \varphi_{11}^{\omega}}{\partial y}=\Delta x \frac{\partial \Phi_{11}^{\omega}}{\partial \beta} \\
\frac{\partial^{2} \varphi_{00}^{\omega}}{\partial x^{2}}=\frac{1}{\Delta x^{2}} \frac{\partial^{2} \Phi_{00}^{\omega}}{\partial \alpha^{2}} & \frac{\partial^{2} \varphi_{10}^{\omega}}{\partial x^{2}}=\frac{1}{\Delta x} \frac{\partial^{2} \Phi_{10}^{\omega}}{\partial \alpha^{2}} & \frac{\partial^{2} \varphi_{01}^{\omega}}{\partial x^{2}}=\frac{\Delta y}{\Delta x^{2}} \frac{\partial^{2} \Phi_{01}^{\omega}}{\partial \alpha^{2}} & \frac{\partial^{2} \varphi_{11}^{\omega}}{\partial x^{2}}=\frac{\Delta y}{\Delta x} \frac{\partial^{2} \Phi_{11}^{\omega}}{\partial \alpha^{2}} \\
\frac{\partial^{2} \varphi_{00}^{\omega}}{\partial y^{2}}=\frac{1}{\Delta y^{2}} \frac{\partial^{2} \Phi_{00}^{\omega}}{\partial \beta^{2}} & \frac{\partial^{2} \varphi_{10}^{\omega}}{\partial y^{2}}=\frac{\Delta x}{\Delta y^{2}} \frac{\partial^{2} \Phi_{10}^{\omega}}{\partial \beta^{2}} & \frac{\partial^{2} \varphi_{01}^{\omega}}{\partial y^{2}}=\frac{1}{\Delta y} \frac{\partial^{2} \Phi_{01}^{\omega}}{\partial \beta^{2}} & \frac{\partial^{2} \varphi_{11}^{\omega}}{\partial y^{2}}=\frac{\Delta x}{\Delta y} \frac{\partial^{2} \Phi_{11}^{\omega}}{\partial \beta^{2}}
\end{array}
$$

With all these tools, it is possible to evaluate the integral within a span of rectangular element.

\section{A.13 Domain with curved boundaries and irregularly shaped mesh: Demonstration with 2-d serendipity bicubic Hermitian basis functions}

For most of this thesis, numerical simulations are conveniently done on square mesh, unnecessitating longer expansions by chain rule for several derivations. However, if one is to investigate the phenomena in curved domain, full expansions cannot not be simplified as much. Furthermore, more complications arise with the boundary conditions. As can be seen in other sections, the wetting boundary condition, more specifically, the first order derivative of the concentration in the direction normal to the surface, must be imposed by forcing some constants unchanged upon linear matrix division. This requires that these constants represent the derivatives of the concentration normal to the surface. Ultimately, the basis functions corresponding to those constants must be twisted in global coordinates accordingly. 
Furthermore, the mixed terms will cause complications in the curved boundaries, as the derivative in Cartesian coordinate is no longer appropriate. Therefore, bicubic Hermitian basis functions with serendipity is chosen so that the mixed terms can be dropped. In this chapter, the idea is demonstrated in 2-d. The idea can be extended to 3-d.

2-d bicubic Hermitian basis functions are derived in similar manner as seen in previous sections. The weights are distributed as following:

$$
c(\alpha, \beta)=\sum_{\tau=\{00,10,01\}} \sum_{\omega=\{00,10,01,11\}} c_{\tau}^{\omega} \Phi_{\tau}^{\omega} \text { where } c_{\tau}^{\omega} \in \mathbb{R}
$$

Where each basis function is the polynomial of the form with 12 constraints:

$$
\begin{aligned}
\Phi_{\tau}^{\omega}=P(\alpha, \beta) & =c_{1}+c_{2} \alpha+c_{3} \beta+c_{4} \alpha^{2}+c_{5} \beta^{2}+c_{6} \alpha \beta+c_{7} \alpha^{3}+c_{8} \beta^{3} \\
& +c_{9} \alpha^{2} \beta+c_{10} \alpha \beta^{2}+c_{11} \alpha^{3} \beta+c_{12} \alpha \beta^{3}
\end{aligned}
$$

Where the constants are as shown below:

\begin{tabular}{|c|c|c|c|c|c|c|c|c|c|c|c|c|}
\hline & $c_{1}$ & $c_{2}$ & $c_{3}$ & $c_{4}$ & $c_{5}$ & $c_{6}$ & $c_{7}$ & $c_{8}$ & $c_{9}$ & $c_{10}$ & $c_{11}$ & $c_{12}$ \\
\hline$\Phi_{00}^{00}$ & 1 & 0 & 0 & -3 & -3 & -1 & 2 & 2 & 3 & 3 & -2 & -2 \\
\hline$\Phi_{10}^{00}$ & 0 & 1 & 0 & -2 & 0 & -1 & 1 & 0 & 2 & 0 & -1 & 0 \\
\hline$\Phi_{01}^{00}$ & 0 & 0 & 1 & 0 & -2 & -1 & 0 & 1 & 0 & 2 & 0 & -1 \\
\hline$\Phi_{00}^{10}$ & 0 & 0 & 0 & 3 & 0 & 1 & -2 & 0 & -3 & -3 & 2 & 2 \\
\hline$\Phi_{10}^{10}$ & 0 & 0 & 0 & -1 & 0 & 0 & 1 & 0 & 1 & 0 & -1 & 0 \\
\hline$\Phi_{01}^{10}$ & 0 & 0 & 0 & 0 & 0 & 1 & 0 & 0 & 0 & -2 & 0 & 1 \\
\hline$\Phi_{00}^{01}$ & 0 & 0 & 0 & 0 & 3 & 1 & 0 & -2 & -3 & -3 & 2 & 2 \\
\hline$\Phi_{10}^{01}$ & 0 & 0 & 0 & 0 & 0 & 1 & 0 & 0 & -2 & 0 & 1 & 0 \\
\hline$\Phi_{01}^{01}$ & 0 & 0 & 0 & 0 & -1 & 0 & 0 & 1 & 0 & 1 & 0 & -1 \\
\hline$\Phi_{00}^{11}$ & 0 & 0 & 0 & 0 & 0 & -1 & 0 & 0 & 3 & 3 & -2 & -2 \\
\hline$\Phi_{10}^{11}$ & 0 & 0 & 0 & 0 & 0 & 0 & 0 & 0 & -1 & 0 & 1 & 0 \\
\hline$\Phi_{01}^{11}$ & 0 & 0 & 0 & 0 & 0 & 0 & 0 & 0 & 0 & -1 & 0 & 1 \\
\hline
\end{tabular}


These are the basis functions to be worked on in this section. Leon Lapidus [78] has related global basis functions to local basis functions as following:

$$
\begin{gathered}
\varphi_{00}^{\omega}=\Phi_{00}^{\omega} \\
\varphi_{10}^{\omega}=\Phi_{10}^{\omega} \frac{\partial x^{\prime}}{\partial \alpha}+\Phi_{01}^{\omega} \frac{\partial x^{\prime}}{\partial \beta} \\
\varphi_{01}^{\omega}=\Phi_{10}^{\omega} \frac{\partial y^{\prime}}{\partial \alpha}+\Phi_{01}^{\omega} \frac{\partial y^{\prime}}{\partial \beta}
\end{gathered}
$$

The above equations serve to twist the basis function along $x^{\prime}$ and $y^{\prime}$ axis upon mapping. Here, the sign " " " is to indicate that this axis of $x^{\prime}$ and $y$ ' have been twisted, or rotated, from the original axis of $x$ and $y$. These will be explained later. First, one needs to determine the derivatives of global basis functions with respect to each component in local coordinate. By expanding the above, one obtains the following:

$$
\begin{gathered}
\frac{\partial \varphi_{00}^{\omega}}{\partial \alpha}=\frac{\partial \Phi_{00}^{\omega}}{\partial \alpha} ; \frac{\partial \varphi_{00}^{\omega}}{\partial \beta}=\frac{\partial \Phi_{00}^{\omega}}{\partial \beta} \\
\frac{\partial^{2} \varphi_{00}^{\omega}}{\partial \alpha^{2}}=\frac{\partial^{2} \Phi_{00}^{\omega}}{\partial \alpha^{2}} ; \frac{\partial^{2} \varphi_{00}^{\omega}}{\partial \beta^{2}}=\frac{\partial^{2} \Phi_{00}^{\omega}}{\partial \beta^{2}} ; \frac{\partial^{2} \varphi_{00}^{\omega}}{\partial \alpha \partial \beta}=\frac{\partial^{2} \Phi_{00}^{\omega}}{\partial \alpha \partial \beta} \\
\frac{\partial \varphi_{10}^{\omega}}{\partial \alpha}=\frac{\partial \Phi_{10}^{\omega}}{\partial \alpha} \frac{\partial x^{\prime}}{\partial \alpha}+\Phi_{10}^{\omega} \frac{\partial^{2} x^{\prime}}{\partial \alpha^{2}}+\frac{\partial \Phi_{01}^{\omega}}{\partial \alpha} \frac{\partial x^{\prime}}{\partial \beta}+\Phi_{01}^{\omega} \frac{\partial^{2} x^{\prime}}{\partial \alpha \partial \beta} \\
\frac{\partial \varphi_{10}^{\omega}}{\partial \beta}=\frac{\partial \Phi_{10}^{\omega}}{\partial \beta} \frac{\partial x^{\prime}}{\partial \alpha}+\Phi_{10}^{\omega} \frac{\partial^{2} x^{\prime}}{\partial \alpha \partial \beta}+\frac{\partial \Phi_{01}^{\omega}}{\partial \beta} \frac{\partial x^{\prime}}{\partial \beta}+\Phi_{01}^{\omega} \frac{\partial^{2} x^{\prime}}{\partial \beta^{2}} \\
\frac{\partial^{2} \varphi_{10}^{\omega}}{\partial \alpha^{2}}=\frac{\partial^{2} \Phi_{10}^{\omega}}{\partial \alpha^{2}} \frac{\partial x^{\prime}}{\partial \alpha}+2 \frac{\partial \Phi_{10}^{\omega}}{\partial \alpha} \frac{\partial^{2} x^{\prime}}{\partial \alpha^{2}}+\Phi_{10}^{\omega} \frac{\partial^{3} x^{\prime}}{\partial \alpha^{3}}+\frac{\partial^{2} \Phi_{01}^{\omega}}{\partial \alpha^{2}} \frac{\partial x^{\prime}}{\partial \beta}+2 \frac{\partial \Phi_{01}^{\omega}}{\partial \alpha} \frac{\partial^{2} x^{\prime}}{\partial \alpha \partial \beta} \\
+\Phi_{01}^{\omega} \frac{\partial^{3} x^{\prime}}{\partial \alpha^{2} \partial \beta} \\
\frac{\partial^{2} \varphi_{10}^{\omega}}{\partial \beta^{2}}=\frac{\partial^{2} \Phi_{10}^{\omega}}{\partial \beta^{2}} \frac{\partial x^{\prime}}{\partial \alpha}+2 \frac{\partial \Phi_{10}^{\omega}}{\partial \beta} \frac{\partial^{2} x^{\prime}}{\partial \alpha \partial \beta}+\Phi_{10}^{\omega} \frac{\partial^{3} x^{\prime}}{\partial \alpha \partial \beta^{2}}+\frac{\partial^{2} \Phi_{01}^{\omega}}{\partial \beta^{2}} \frac{\partial x^{\prime}}{\partial \beta} \\
+2 \frac{\partial \Phi_{01}^{\omega}}{\partial \beta} \frac{\partial^{2} x^{\prime}}{\partial \beta^{2}}+\Phi_{01}^{\omega} \frac{\partial^{3} x^{\prime}}{\partial \beta^{3}}
\end{gathered}
$$




$$
\begin{gathered}
\frac{\partial^{2} \varphi_{10}^{\omega}}{\partial \alpha \partial \beta}=\frac{\partial^{2} \Phi_{10}^{\omega}}{\partial \alpha \partial \beta} \frac{\partial x^{\prime}}{\partial \alpha}+\frac{\partial \Phi_{10}^{\omega}}{\partial \alpha} \frac{\partial^{2} x^{\prime}}{\partial \alpha \partial \beta}+\frac{\partial \Phi_{10}^{\omega}}{\partial \beta} \frac{\partial^{2} x^{\prime}}{\partial \alpha^{2}}+\Phi_{10}^{\omega} \frac{\partial^{3} x^{\prime}}{\partial \alpha^{2} \partial \beta}+\frac{\partial^{2} \Phi_{01}^{\omega}}{\partial \alpha \partial \beta} \frac{\partial x^{\prime}}{\partial \beta} \\
+\frac{\partial \Phi_{01}^{\omega}}{\partial \alpha} \frac{\partial^{2} x^{\prime}}{\partial \beta^{2}}+\frac{\partial \Phi_{01}^{\omega}}{\partial \beta} \frac{\partial^{2} x^{\prime}}{\partial \alpha \partial \beta}+\Phi_{01}^{\omega} \frac{\partial^{3} x^{\prime}}{\partial \alpha \partial \beta^{2}} \\
\frac{\partial \varphi_{01}^{\omega}}{\partial \alpha}=\frac{\partial \Phi_{10}^{\omega}}{\partial \alpha} \frac{\partial y^{\prime}}{\partial \alpha}+\Phi_{10}^{\omega} \frac{\partial^{2} y^{\prime}}{\partial \alpha^{2}}+\frac{\partial \Phi_{01}^{\omega}}{\partial \alpha} \frac{\partial y^{\prime}}{\partial \beta}+\Phi_{01}^{\omega} \frac{\partial^{2} y^{\prime}}{\partial \alpha \partial \beta} \\
\frac{\partial \varphi_{01}^{\omega}}{\partial \beta}=\frac{\partial \Phi_{10}^{\omega}}{\partial \beta} \frac{\partial y^{\prime}}{\partial \alpha}+\Phi_{10}^{\omega} \frac{\partial^{2} y^{\prime}}{\partial \alpha \partial \beta}+\frac{\partial \Phi_{01}^{\omega}}{\partial \beta} \frac{\partial y^{\prime}}{\partial \beta}+\Phi_{01}^{\omega} \frac{\partial^{2} y^{\prime}}{\partial \beta^{2}} \\
+\frac{\partial^{2} \varphi_{01}^{\omega}}{\partial \alpha^{2}}=\frac{\partial^{2} \Phi_{10}^{\omega}}{\partial \alpha^{2}} \frac{\partial y^{\prime}}{\partial \alpha}+2 \frac{\partial \Phi_{10}^{\omega}}{\partial \alpha} \frac{\partial^{2} y^{\prime}}{\partial \alpha^{2}}+\Phi_{10}^{\omega} \frac{\partial^{3} y^{\prime}}{\partial \alpha^{3}}+\frac{\partial^{2} \Phi_{01}^{\omega}}{\partial \alpha^{2}} \frac{\partial y^{\prime}}{\partial \beta}+2 \frac{\partial \Phi_{01}^{\omega}}{\partial \alpha} \frac{\partial^{2} y^{\prime}}{\partial \alpha \partial \beta} \\
+\frac{\partial^{2} \varphi_{01}^{\omega}}{\partial \beta^{2}}=\frac{\partial^{2} \Phi_{10}^{\omega}}{\partial \beta^{2}} \frac{\partial \Phi^{\prime}}{\partial \alpha}+2 \frac{\partial \Phi_{10}^{\omega}}{\partial \beta} \frac{\partial^{2} y^{\prime}}{\partial \alpha \partial \beta}+\Phi_{10}^{\omega} \frac{\partial^{3} y^{\prime}}{\partial \alpha \partial \beta^{2}}+\frac{\partial^{2} \Phi_{01}^{\omega}}{\partial \beta^{2}} \frac{\partial y^{\prime}}{\partial \beta} \\
+2 \frac{\partial \Phi_{01}^{\omega}}{\partial \beta} \frac{\partial^{2} y^{\prime}}{\partial \beta^{2}}+\Phi_{01}^{\omega} \frac{\partial^{3} y^{\prime}}{\partial \beta^{3}} \\
\frac{\partial^{2} \varphi_{01}^{\omega}}{\partial \alpha \partial \beta}=\frac{\partial^{2} \Phi_{10}^{\omega}}{\partial \alpha \partial \beta} \frac{\partial y^{\prime}}{\partial \alpha}+\frac{\partial \Phi_{10}^{\omega}}{\partial \alpha} \frac{\partial^{2} y^{\prime}}{\partial \alpha \partial \beta}+\frac{\partial \Phi_{10}^{\omega}}{\partial \beta} \frac{\partial^{2} y^{\prime}}{\partial \alpha^{2}}+\Phi_{10}^{\omega} \frac{\partial^{3} y^{\prime}}{\partial \alpha^{2} \partial \beta}+\frac{\partial^{2} \Phi_{01}^{\omega}}{\partial \alpha \partial y^{\prime}} \frac{\partial^{3} y^{\prime}}{\partial \alpha \partial \beta^{2}} \\
+
\end{gathered}
$$

For linear isoparametric mapping, many terms in the equations above cancel to zero. Though linear isoparametric mapping can be used given that the mesh is fine enough to represent curvature, other appropriate types of mapping representing the curvatures within an element are recommended. Once these derivatives are computed, one may obtain the derivatives in global component by using the same way as in previous sections, i.e. as in (A.12.7) given (A.12.6).

Next, the rotation of the axis is discussed. Present model has required that, upon linear matrix division, some constants representing the first derivative of the solution normal to the surface. Therefore, corresponding basis functions at the boundary must be twisted along this direction. A convenient tool for this is rotation axis. For 2-d, typically the new coordinate is related to original coordinate as following: 


$$
\begin{aligned}
& \left(\begin{array}{l}
x^{\prime} \\
y^{\prime}
\end{array}\right)=\left(\begin{array}{cc}
\cos \theta & \sin \theta \\
-\sin \theta & \cos \theta
\end{array}\right)\left(\begin{array}{l}
x \\
y
\end{array}\right) \\
& \left(\begin{array}{l}
x \\
y
\end{array}\right)=\left(\begin{array}{cc}
\cos \theta & -\sin \theta \\
\sin \theta & \cos \theta
\end{array}\right)\left(\begin{array}{l}
x^{\prime} \\
y^{\prime}
\end{array}\right)
\end{aligned}
$$

Where $\theta$ is the angle of newly twisted coordinate. This way, the derivatives of the twisted global coordinate with respect to local coordinate can be determined as following:

$$
\begin{aligned}
\left(\begin{array}{l}
\frac{\partial x^{\prime}}{\partial \alpha} \\
\frac{\partial y^{\prime}}{\partial \alpha}
\end{array}\right) & =\left(\begin{array}{cc}
\cos \theta & \sin \theta \\
-\sin \theta & \cos \theta
\end{array}\right)\left(\begin{array}{l}
\frac{\partial x}{\partial \alpha} \\
\frac{\partial y}{\partial \alpha}
\end{array}\right) \\
\left(\begin{array}{c}
\frac{\partial x^{\prime}}{\partial \beta} \\
\frac{\partial y^{\prime}}{\partial \beta}
\end{array}\right) & =\left(\begin{array}{cc}
\cos \theta & \sin \theta \\
-\sin \theta & \cos \theta
\end{array}\right)\left(\begin{array}{l}
\frac{\partial x}{\partial \beta} \\
\frac{\partial y}{\partial \beta}
\end{array}\right) \\
\left(\begin{array}{c}
\frac{\partial^{2} x^{\prime}}{\partial \beta^{2}} \\
\frac{\partial^{2} y^{\prime}}{\partial \beta^{2}}
\end{array}\right) & =\left(\begin{array}{cc}
\cos \theta & \sin \theta \\
-\sin \theta & \cos \theta
\end{array}\right)\left(\begin{array}{l}
\frac{\partial^{2} x}{\partial \beta^{2}} \\
\frac{\partial^{2} y}{\partial \beta^{2}}
\end{array}\right) \\
\left(\begin{array}{c}
\partial^{3} x^{\prime} \\
\partial \alpha \partial \beta^{2}
\end{array}\right. & =\left(\begin{array}{cc}
\cos \theta & \sin \theta \\
-\sin \theta & \cos \theta
\end{array}\right)\left(\begin{array}{c}
\frac{\partial^{3} x}{\partial \alpha \partial \beta^{2}} \\
\frac{\partial^{3} y}{\partial \alpha \partial y^{\prime}}
\end{array}\right)
\end{aligned}
$$

Now, it has been shown how to deliberately twist the basis functions so that they can represent the directional derivative in the desired direction. On the side note, however, it comes with its own weakness. If the basis functions as twisted too differently at different nodes within one element, it may lead to complications in global basis functions, causing determining of absolute weights and their derivatives less realistic. Thus, one should always be wary of advantages and 
disadvantages by fully understanding the numerical methods that should be used. Once the numerical method has been fully understood, one may lay out several potential strategies for countering problems, such as increasing the mesh size to better account each structure at the expense of computational cost or limit the studies to only structures that are mainly low in frequency. 


\section{Appendix B Complimentary table and figures}

This section serves to help readers to understand some structures of the thesis by summarizing all simulations into Table B - 1 . Keep in mind that, for this entire thesis, $\Psi=1$ and $n_{1}=1$. For neatness, the types of several parameters are defined. Each type is unique for given 1 or more values (e.g. Concentration gradient of type A is defined by two values, $c_{o_{1}}$ and $c_{o_{2}}$, which are the initial concentration without noise at $x=0$ and $x=1$, respectively.). The types of relevant parameters are defined as following:

- Concentration gradient types:

o Type A: Nonlinear concentration gradient obeying the following equation:

$$
c_{o}(x, y)-\eta_{o}=c_{1}+c_{2} x+c_{2} x^{2}+c_{1} x^{3}
$$

Where $c_{1}, c_{2}, c_{3}$, and $c_{4} \in \mathbb{R}$ such that they satisfy the following 4 constraints:

$$
\begin{gathered}
\left.c_{o}\right|_{x=0}=c_{o_{1}} \\
\left.c_{o}\right|_{x=1}=c_{o_{2}} \\
\left.\nabla c_{o}\right|_{x=0}=\left.\nabla c_{o}\right|_{x=1}=\overrightarrow{0}
\end{gathered}
$$

- Temperature gradient types:

o Type A: Linear temperature gradient obeying the following equation:

$$
T(x, y)=c_{1}+c_{2} x
$$

Where $c_{1}$ and $c_{2} \in \mathbb{R}$ such that they satisfy the following 2 constraints:

$$
\left.T\right|_{x=0}=T_{1}
$$




$$
\left.T\right|_{x=1}=T_{2}
$$

o Type B: Nonlinear temperature gradient obeying the following equation:

$$
T(x, y)=c_{1}+c_{2} x+c_{2} x^{2}+c_{1} x^{3}
$$

Where $c_{1}, c_{2}, c_{3}$, and $c_{4} \in \mathbb{R}$ such that they satisfy the following 4 constraints:

$$
\begin{aligned}
\left.T\right|_{x=0} & =T_{1} \\
\left.T\right|_{x=1} & =T_{2} \\
\left.\nabla T\right|_{x=0} & =\left.\nabla T\right|_{x=1}=\overrightarrow{0}
\end{aligned}
$$

- Polymerization gradient:

o Type A: Nonlinear temperature gradient obeying the following equation:

$$
n_{2}(x, y)=c_{1}+c_{2} x+c_{2} x^{2}+c_{1} x^{3}
$$

Where $c_{1}, c_{2}, c_{3}$, and $c_{4} \in \mathbb{R}$ such that they satisfy the following 4 constraints:

$$
\begin{gathered}
\left.n_{2}\right|_{x=0}=n_{21} \\
\left.n_{2}\right|_{x=1}=n_{22} \\
\left.\nabla n_{2}\right|_{x=0}=\left.\nabla n_{2}\right|_{x=1}=\overrightarrow{0}
\end{gathered}
$$

o Type B: Same as type A except the gradient exists along y-direction instead.

- Wetting patterns

o Type A: Uniform surface potential regardless of domain shape, i.e.

$$
\left.\nabla c\right|_{\partial \Omega} \cdot \hat{n}=h_{1}+g_{1} c
$$

o Type B: Stripe pattern consisting of 8 rectangular regions alternating between $h_{1}$ with $g_{1}$ and $h_{2}$ with $g_{2}$. The wetting surface is located only on the plane $z=1$. 
o Type C: Wetting on two surfaces, $z=0$ and $z=1$ with parameters $h_{1}$ with $g_{1}$ and $h_{2}$ with $g_{2}$, respectively.

o Type D: Wetting on the surface $z=1$ within the circular region centered at $(x, y)=(0.5,0.5)$ with radius of 0.05 . Wetting is specified by $g_{1}$ and $h_{1}$

- Mesh types and their basis functions (Note that every element for this thesis is quadrilateral and no element repeats or overlaps the other, but may only share the edges):

o Type A: 2-d mesh with 256x256 nodes (65536 nodal points in total) spread equally on square domain whose edges are at $(0,0),(1,0),(0,1)$, and $(1,1)$ on $x y$ coordinates. This way, every element has the same size. 2-d bicubic Hermitian basis functions are used.

o Type B: 3-d mesh with 32×32×32 nodes (32768 nodal points in total) spread equally on cubic domain whose edges are at $(0,0,0),(1,0,0),(0,1,0),(1,1,0)$, $(0,0,1),(1,0,1),(0,1,1),(1,1,1)$ on xyz coordinates. This way, every element has the same size. 3-d bicubic Hermitian basis functions with serendipity are used.

o Type C: 2 -d mesh with $256 \times 256$ nodes (65536 nodal points in total) on a circular domain whose area is 1 unit squared and the origin is centered at $(0,0)$ on $x y$ coordinates. Nodal points are defined as following: First, all points are spread equally on a square domain whose edges are at $(-1,-1),(1,-1),(-1,1)$, and $(1,1)$ on xy coordinates. Each point is then mapped to the circle as following:

$$
\begin{aligned}
& x_{\text {circle }}=\frac{x_{\text {square }}}{\sqrt{\pi}} \sqrt{1-\frac{y_{\text {square }}{ }^{2}}{2}} \\
& y_{\text {circle }}=\frac{y_{\text {square }}}{\sqrt{\pi}} \sqrt{1-\frac{x_{\text {square }}{ }^{2}}{2}}
\end{aligned}
$$

Where the subscript "square" indicates the coordinates defined on square domain as above, and the subscript "circle" indicates the newly mapped coordinates of nodal points, which define mesh Type C. Note that, the basis functions for this type are "twisted" accordingly, as explained in section A.13. 
Table B - 1 summarizes all parameters. If the type is not shown, then the value is the same throughout the entire domain. If the type is shown, then all the values that are necessary to define the parameter are also shown. The quench is classified as critical base on solely average concentration, $c_{\text {ave }}$, and $n_{2}$ given that polymerization gradient does not exists. If the quench is critical, they are indicated underneath values of $c_{\text {ave }} . f_{c}$ is the characteristic frequency computed based on (A.1.22). The local free energy term is as shown on (4.2.24), evaluated at $c_{\text {ave. }}$ This value can be evaluated as real number only in unstable region, thus if the quench is metastable, it will be indicated as such. If the quench is anisotropic with varying $T$ or $n_{2}$, then $f_{c}$ for shallowest and deepest quenches are computed using the values of $T$ and $n_{2}$ that lie within the simulation range.

\begin{tabular}{|c|c|c|c|c|c|c|c|c|c|c|}
\hline Case & $D$ & $c_{\text {ave }}$ & $c_{o}$ & $\left\|\eta_{o}\right\|_{\max }$ & $T$ & $D_{T}$ & $n_{2}$ & Wetting & $f_{c}$ & Mesh \\
\hline 3.A & 5,000 & $\begin{array}{l}0.5 \\
\text { (Critical) }\end{array}$ & Uniform & $10^{-6}$ & 0.3 & - & 1 & None & 5.629831 & Type B \\
\hline $3 . B$ & 5,000 & $\begin{array}{l}0.5 \\
\text { (Critical) }\end{array}$ & Uniform & $10^{-6}$ & 0.3 & - & 1 & $\begin{array}{l}\text { Type B } \\
\begin{array}{l}h_{1}=5 \times 10^{-4} \\
g_{1}=5 \times 10^{-4} \\
h_{1}=-5 \times 10^{-4} \\
g_{1}=-5 \times 10^{-4}\end{array}\end{array}$ & 5.629831 & Type B \\
\hline 3.C & 5,000 & $\begin{array}{l}0.5 \\
\text { (Critical) }\end{array}$ & Uniform & $10^{-6}$ & 0.3 & - & 1 & $\begin{array}{l}\text { Type C } \\
\qquad \begin{array}{c}h_{1}=10^{-3} \\
g_{1}=10^{-3} \\
h_{1}=-10^{-3} \\
g_{1}=-10^{-3}\end{array}\end{array}$ & 5.629831 & Type B \\
\hline 3.D & 5,000 & $\begin{array}{l}0.5 \\
\text { (Critical) }\end{array}$ & Uniform & $10^{-6}$ & 0.3 & - & 1 & $\begin{array}{l}\text { Type D } \\
\begin{array}{c}h_{1}=0.1 \\
g_{1}=0\end{array}\end{array}$ & 5.629831 & Type B \\
\hline 5.1.1.A & 50,000 & 0.690983 & Uniform & $10^{-6}$ & 0.56 & - & 3 & None & 4.020059 & Type A \\
\hline 5.1.1.B & 50,000 & $\begin{array}{l}0.690983 \\
\text { (Critical) }\end{array}$ & $\begin{array}{l}c \text { obtained from } \\
\text { case 5.1.1.A at } t= \\
1.411752 \times 10^{-5}\end{array}$ & $10^{-6}$ & 0.56 & - & 5 & None & 13.012603 & Type A \\
\hline 5.1.1.C & 50,000 & $\begin{array}{l}0.690983 \\
\text { (Critical) }\end{array}$ & $\begin{array}{l}c \text { obtained from } \\
\text { case 5.1.1.A at } t= \\
2.682418 \times 10^{-5}\end{array}$ & $10^{-6}$ & 0.56 & - & 5 & None & 13.012603 & Type A \\
\hline 5.1.1.D & 50,000 & $\begin{array}{l}0.690983 \\
\text { (Critical) }\end{array}$ & $\begin{array}{l}c \text { obtained from } \\
\text { case 5.1.1.A at } t= \\
2.964788 \times 10^{-5}\end{array}$ & $10^{-6}$ & 0.56 & - & 5 & None & 13.012603 & Type A \\
\hline 5.1.2.A & 10,000 & 0.2 & Uniform & $10^{-6}$ & 0.26 & - & 1 & None & 3.818337 & Type A \\
\hline 5.1.2.B & 10,000 & 0.2 & $\begin{array}{l}c \text { obtained from } \\
\text { case 5.1.2.A at } t=\end{array}$ & 0 & 0.29 & - & 1 & None & $\begin{array}{l}\text { Meta- } \\
\text { stable }\end{array}$ & Type A \\
\hline
\end{tabular}




\begin{tabular}{|c|c|c|c|c|c|c|c|c|c|c|}
\hline & & & $3.264183 \times 10^{-5}$ & & & & & & & \\
\hline 5.1.2.C & 10,000 & 0.3 & Uniform & $10^{-6}$ & 0.3 & - & 1 & None & 5.866150 & Type A \\
\hline 5.1.2.D & 10,000 & 0.3 & $\begin{array}{l}c \text { obtained from } \\
\text { case 5.1.2.C at } t= \\
5.318622 \times 10^{-6}\end{array}$ & 0 & 0.35 & - & 1 & None & $\begin{array}{l}\text { Meta- } \\
\text { stable }\end{array}$ & Type A \\
\hline 5.1.2.E & 10,000 & 0.3 & $\begin{array}{l}c \text { obtained from } \\
\text { case 5.1.2.C at } t= \\
5.318622 \times 10^{-6}\end{array}$ & 0 & 0.36 & - & 1 & None & $\begin{array}{l}\text { Meta- } \\
\text { stable }\end{array}$ & Type A \\
\hline 5.2.1.A & 100,000 & 0.3 & Uniform & $10^{-3}$ & $\begin{array}{l}\text { Type B } \\
T_{1}=0.34 \\
T_{2}=0.30\end{array}$ & 0 & 1 & None & $\begin{array}{l}\text { Shallowest: } \\
7.205514 \\
\text { Deepest: } \\
18.550394\end{array}$ & Type A \\
\hline 5.2.3.A & 60,000 & 0.7 & Uniform & $10^{-3}$ & $\begin{array}{l}\text { Type B } \\
T_{1}=0.34 \\
T_{2}=0.30\end{array}$ & 0 & $\begin{array}{l}\text { Type B } \\
n_{2_{1}}=1 \\
n_{2_{2}}=1.3\end{array}$ & None & $\begin{array}{l}\text { Shallowest: } \\
5.581367 \\
\text { Deepest: } \\
19.545122\end{array}$ & Type A \\
\hline 5.3.A & 5,000 & 0.3 & Uniform & $10^{-6}$ & 0.35 & - & 1 & $\begin{array}{l}\text { Type A } \\
\begin{array}{c}h_{1}=-1.0 \\
g_{1}=0\end{array}\end{array}$ & $\begin{array}{l}\text { Meta- } \\
\text { stable }\end{array}$ & Type B \\
\hline 5.4.A & 100,000 & 0.25 & $\begin{array}{l}\text { Type A } \\
\qquad \begin{aligned} c_{o_{1}} & =0.25 \\
c_{o_{2}} & =0.35\end{aligned}\end{array}$ & $10^{-3}$ & 0.35 & - & 1 & None & $\begin{array}{l}\text { Meta- } \\
\text { stable }\end{array}$ & Type A \\
\hline 5.5.A & 6,000 & $\begin{array}{l}0.5 \\
\text { (Critical) }\end{array}$ & Uniform & $10^{-6}$ & 0.3 & - & 1 & None & 6.167171 & Type C \\
\hline 5.5.B & 6,000 & $\begin{array}{l}0.5 \\
\text { (Critical) }\end{array}$ & Uniform & $10^{-6}$ & 0.3 & - & 1 & $\begin{array}{l}\text { Type A } \\
\qquad \begin{array}{c}h_{1}=10^{-7} \\
g_{1}=0\end{array}\end{array}$ & 6.167171 & Type C \\
\hline 5.5.C & 6,000 & $\begin{array}{l}0.5 \\
\text { (Critical) }\end{array}$ & Uniform & $10^{-6}$ & 0.3 & - & 1 & $\begin{array}{l}\text { Type A } \\
\begin{array}{c}h_{1}=10^{-6} \\
g_{1}=0\end{array}\end{array}$ & 6.167171 & Type C \\
\hline 5.5.D & 6,000 & $\begin{array}{l}0.5 \\
\text { (Critical) }\end{array}$ & Uniform & $10^{-6}$ & 0.3 & - & 1 & $\begin{array}{l}\text { Type A } \\
\begin{array}{c}h_{1}=10^{-5} \\
g_{1}=0\end{array}\end{array}$ & 6.167171 & Type C \\
\hline 5.5.E & 6,000 & $\begin{array}{l}0.5 \\
\text { (Critical) }\end{array}$ & Uniform & $10^{-6}$ & 0.3 & - & 1 & $\begin{array}{l}\text { Type A } \\
\qquad \begin{array}{c}h_{1}=10^{-4} \\
g_{1}=0\end{array}\end{array}$ & 6.167171 & Type C \\
\hline 5.5.F & 6,000 & $\begin{array}{l}0.5 \\
\text { (Critical) }\end{array}$ & Uniform & $10^{-6}$ & 0.3 & - & 1 & $\begin{array}{l}\text { Type A } \\
\qquad \begin{array}{c}h_{1}=10^{-3} \\
g_{1}=0\end{array}\end{array}$ & 6.167171 & Type C \\
\hline
\end{tabular}

Table B - 1 Parameters used for all simulations discussed in this thesis 

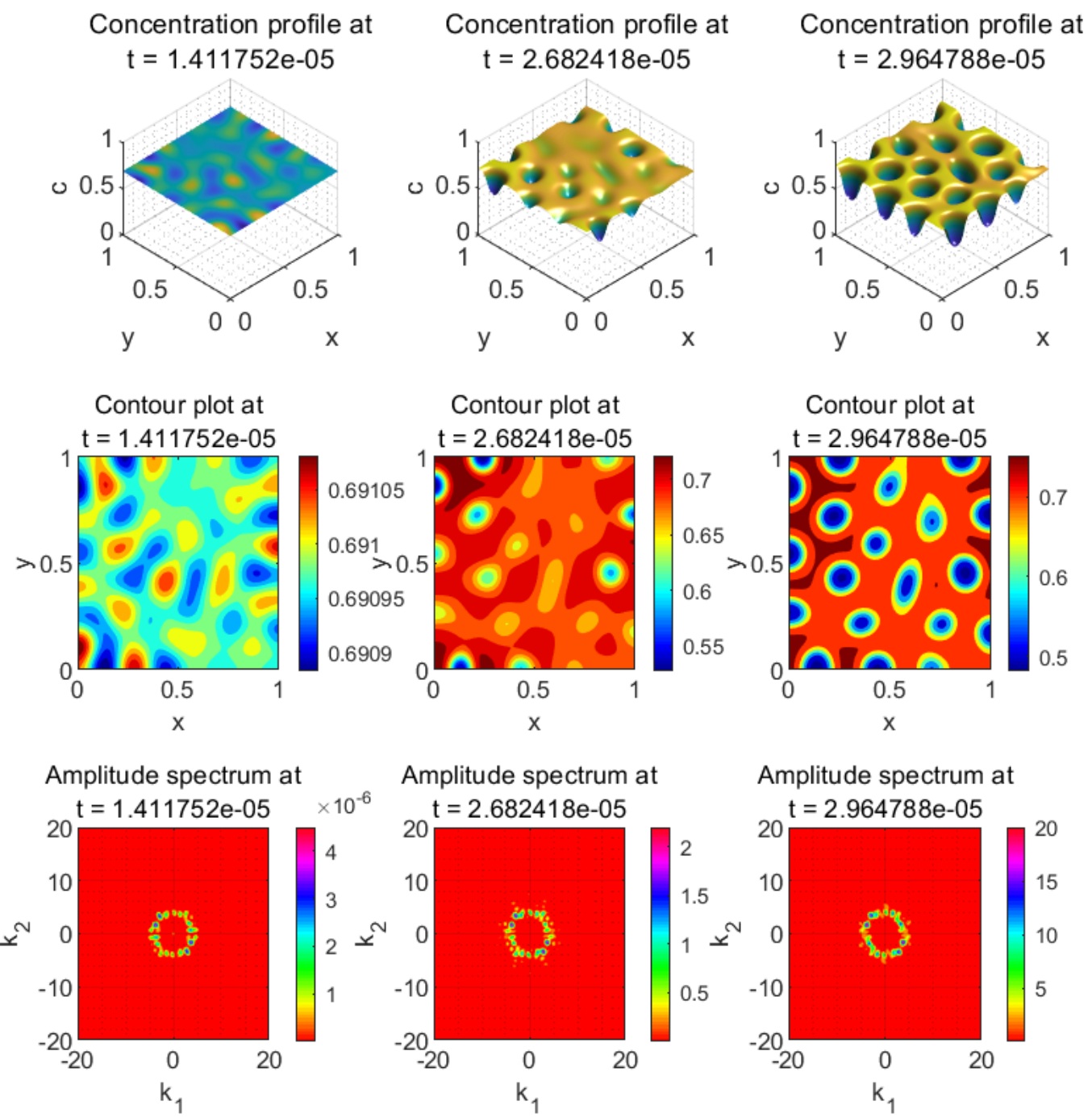

Amplitude spectrum at

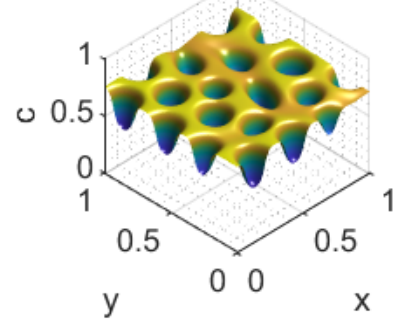

Contour plot at

Contour plot at

Contour plot at

$\mathrm{t}=2.964788 \mathrm{e}-05$

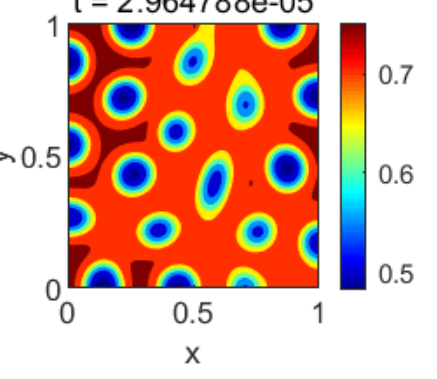

Concentration

$\mathrm{t}=2.682418 \mathrm{e}-05$

Amplitude spectrum at
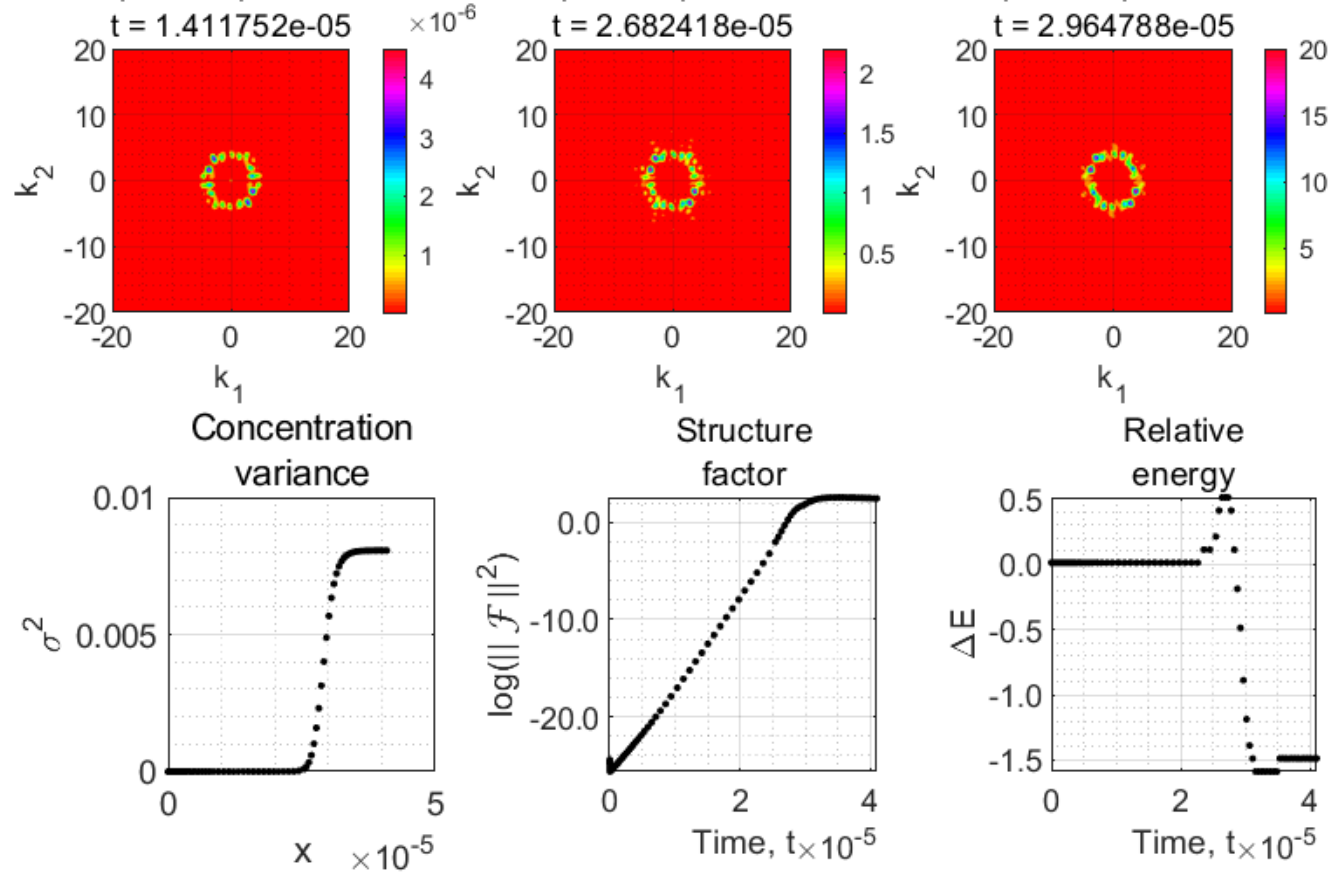

Relative

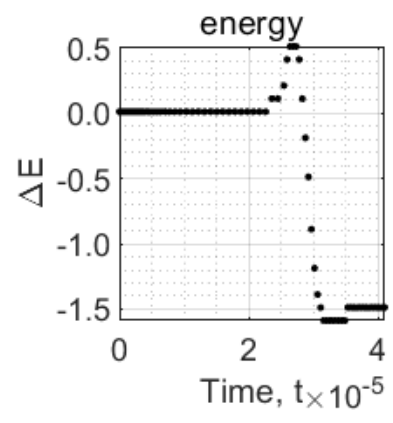

Figure B - 1 Overview of Case 5.1.1.A 

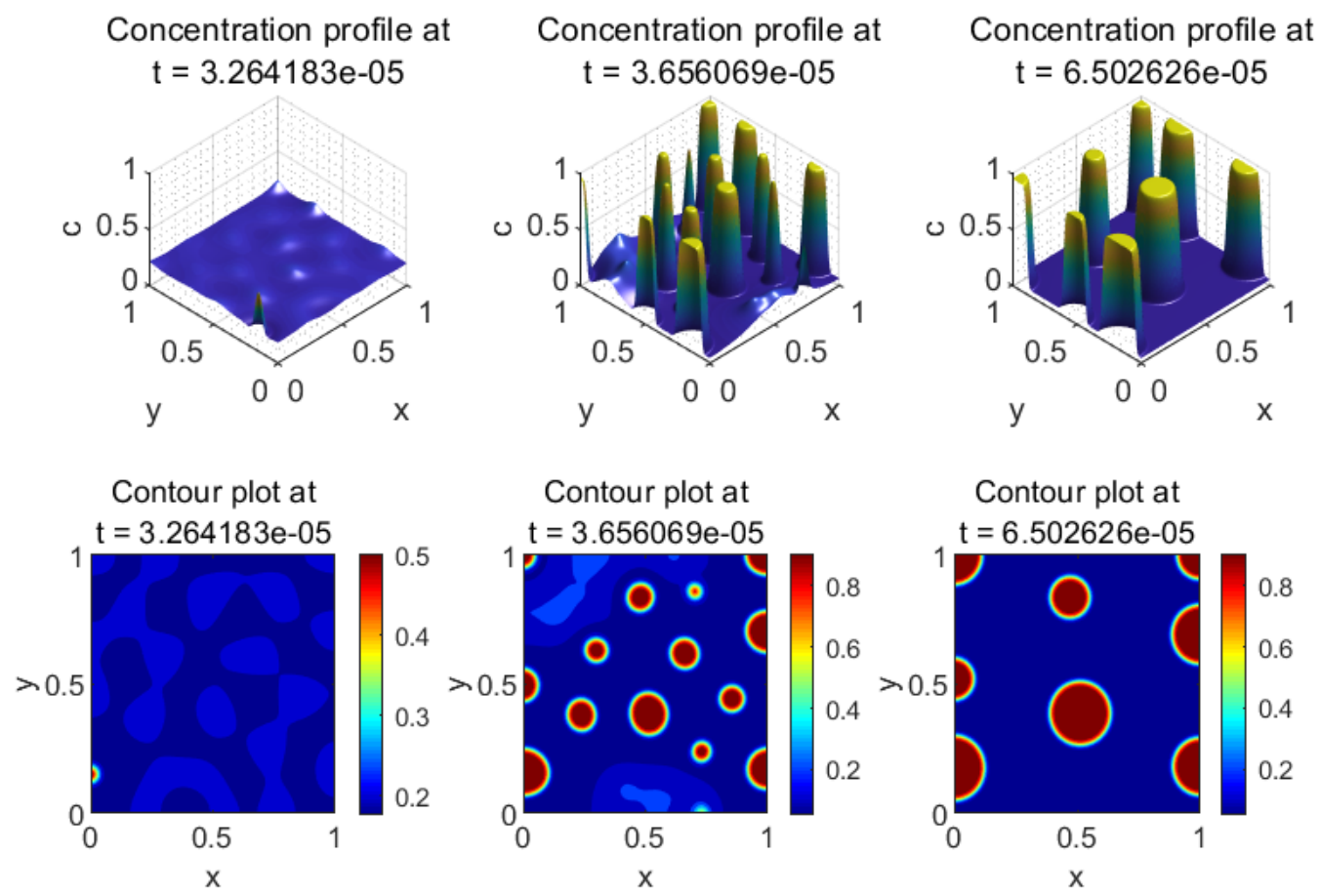

Contour plot at

Contour plot at

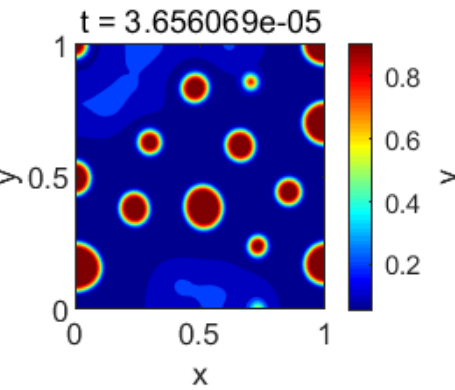

$\mathrm{t}=6.502626 \mathrm{e}-05$

Amplitude spectrum at

Amplitude spectrum at
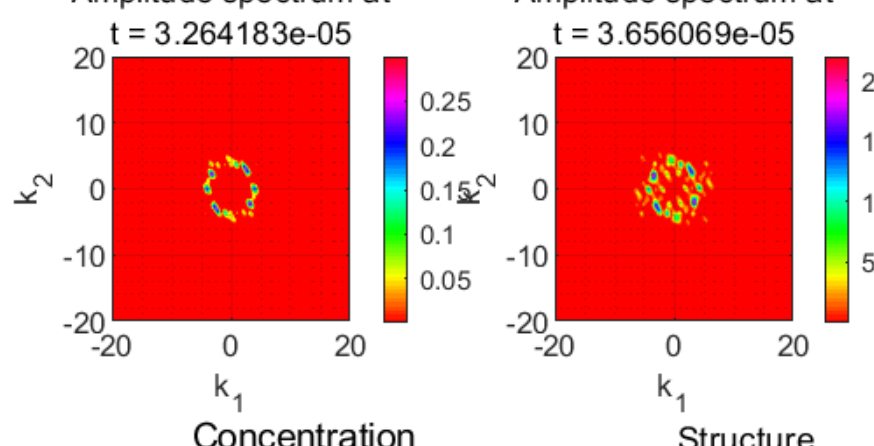

Amplitude spectrum at

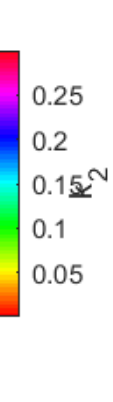

Concentration
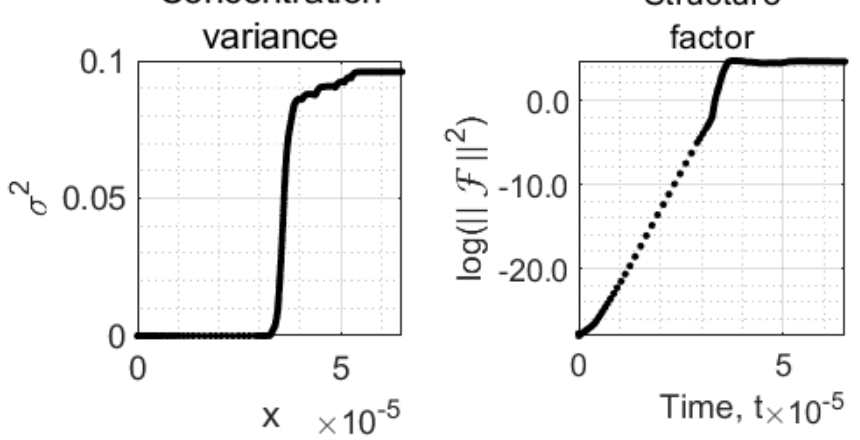

$t=6.502626 \mathrm{e}-05$
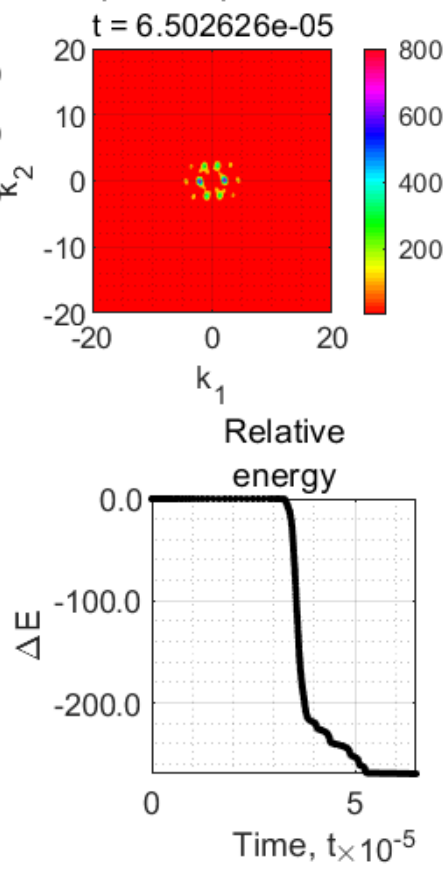

Figure B - 2 Overview of Case 5.1.2.A 

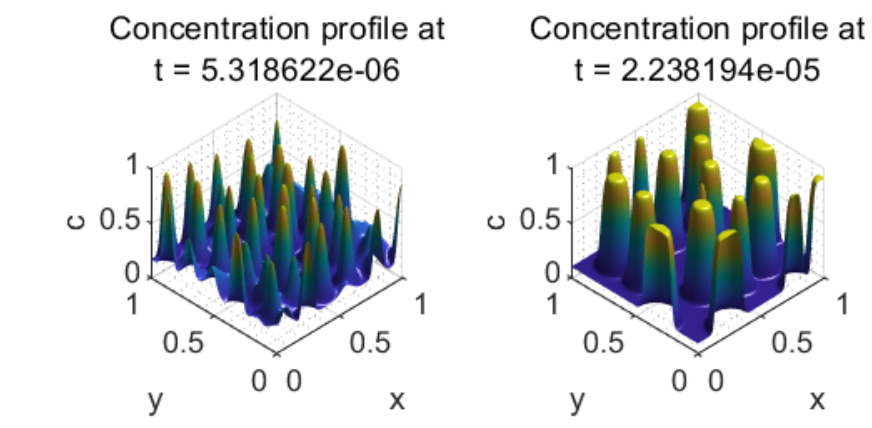
Concentration profile at $\mathrm{t}=2.188111 \mathrm{e}-03$
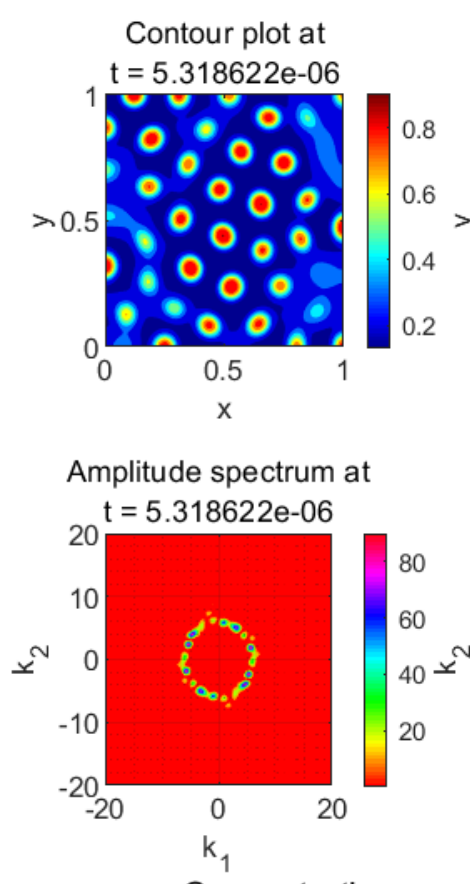

Amplitude spectrum at

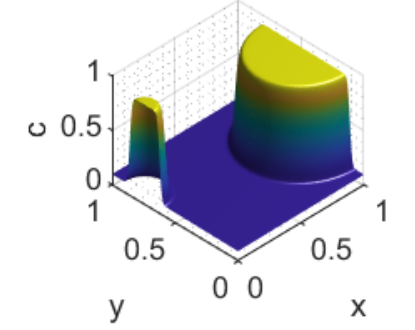

Contour plot at

Contour plot at $t=2.238194 \mathrm{e}-05$
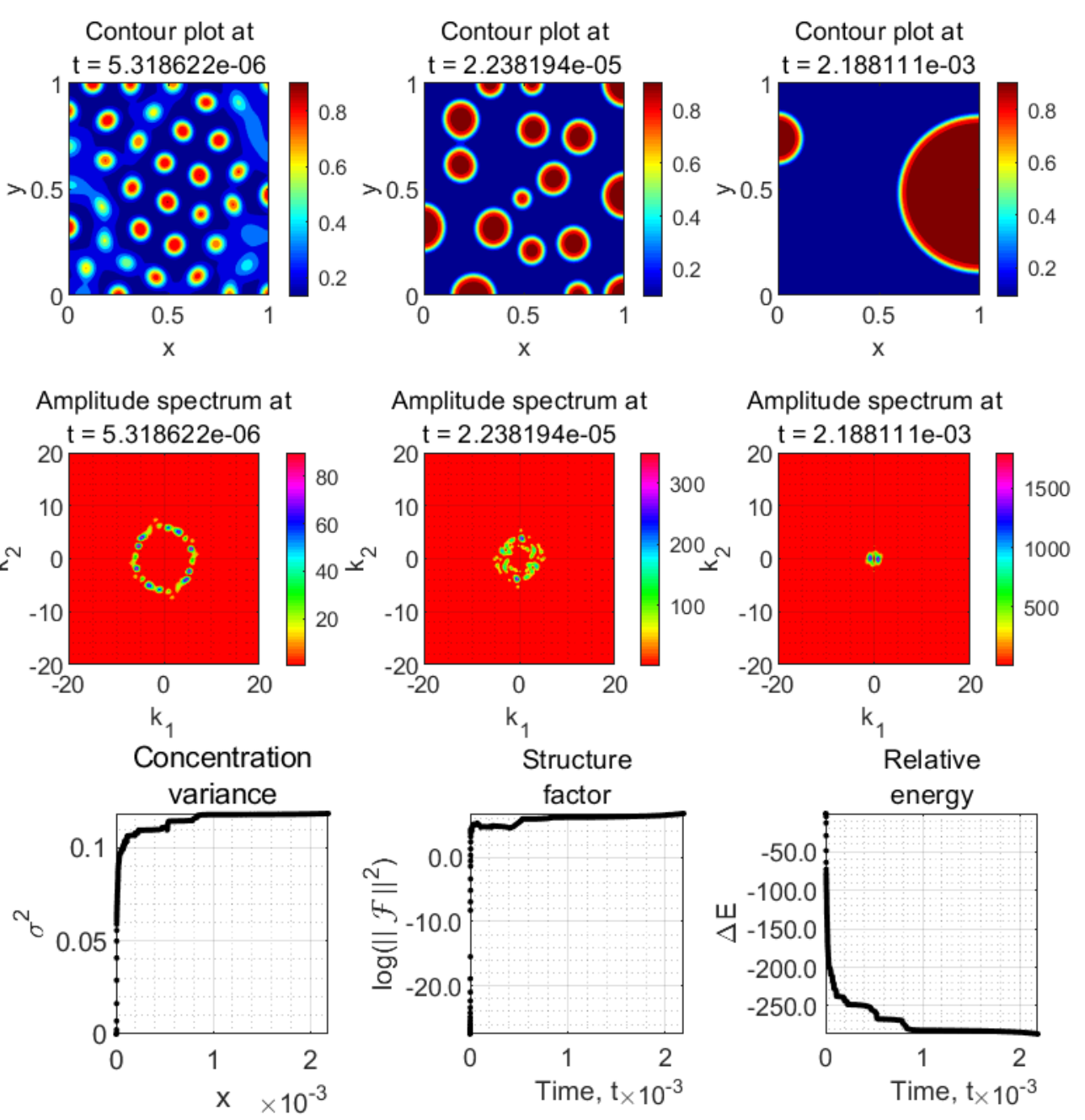

Figure B - 3 Overview of Case 5.1.2.C 

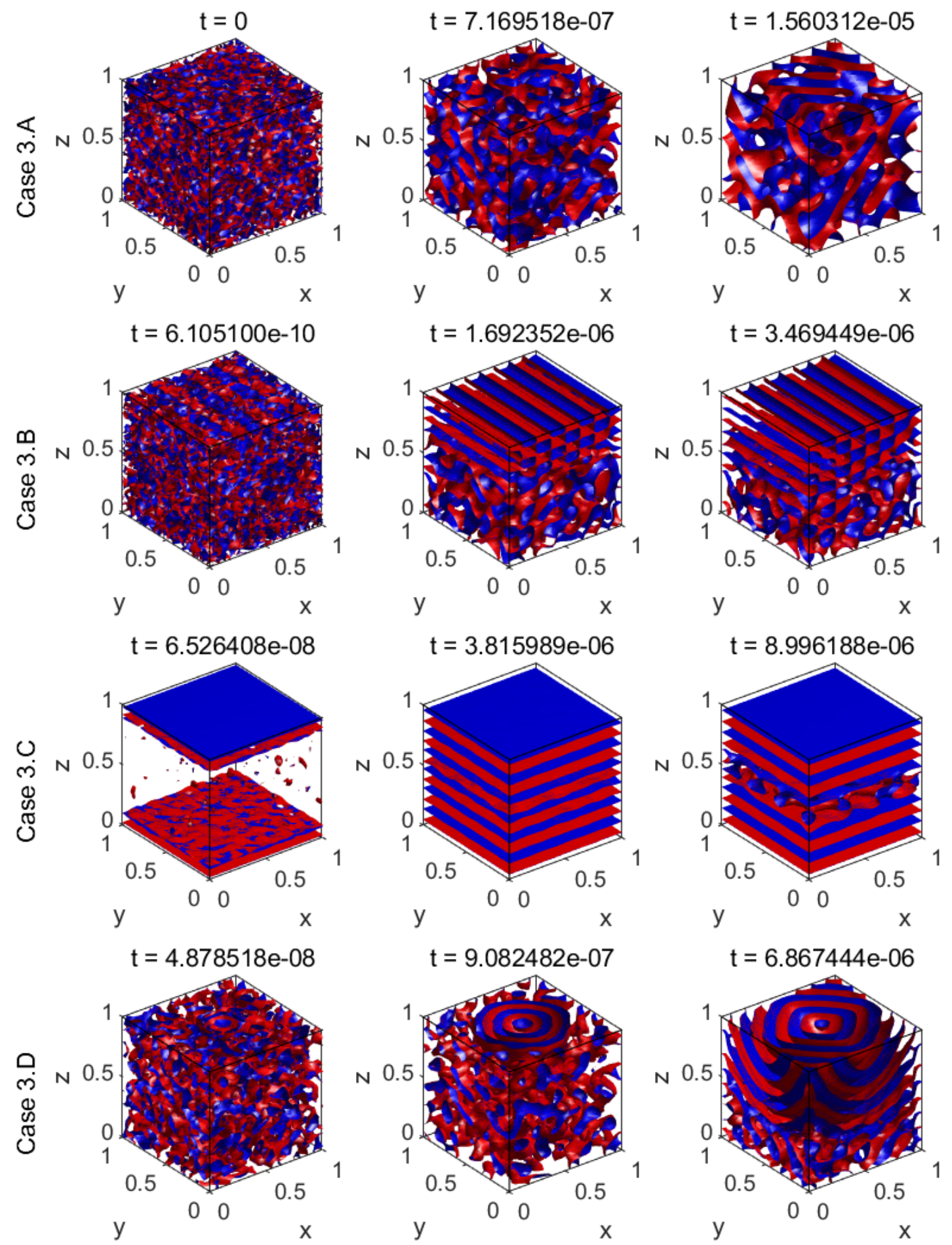

Figure B - 4 Isosurface plots of cases 3.A, 3.B, 3.C, and 3.D 